WSRC-STI-2006-00096, Rev. 1

Key Words:

Environment

Remediation

Retention:

Permanent

\title{
Scenarios Evaluation Tool for Chlorinated Solvent MNA
}

\author{
(A Research Study of the Monitored Natural Attenuation/Enhanced \\ Attenuation for Chlorinated Solvents Technology Alternative Project)
}

October 2, 2006

Washington Savannah River Company
Savannah River Site
Aiken, SC 29808
$\begin{aligned} & \text { Prepared for the U.S. Department of Energy } \\ & \text { Under Contract Number DEAC09-96- } \\ & \text { SR18500 }\end{aligned}$




\section{DISCLAIMER}

Preparation of this report was coordinated by Washington Savannah River Company (WSRC) for the United States Department of Energy (US DOE) under Contract No. DE-AC09-96SR18500. Extensive effort was made by the authors to assure the accuracy of the contents and interpretation. However, the USDOE nor WSRC, nor any of their employees makes any warranty, expressed or implied, or assumes any legal liability or responsibility for accuracy, completeness, or usefulness, of any information, apparatus, or product or process disclosed herein or represents that its use will not infringe privately owned rights. Reference herein to any specific commercial product, process, or service by trade name, trademark, name, manufacturer or otherwise does not necessarily constitute or imply endorsement, recommendation, or favoring of same by Washington Savannah River Company or by the United States Government or any agency thereof. The views and opinions of the authors expressed herein do not necessarily state or reflect those of the United States Government or any agency thereof.

Printed in the United States of America

Prepared For

U.S. Department of Energy 
WSRC-STI-2006-00096, Rev. 1

October 2, 2006

Page iii of vi

Key Words:

Environment

Remediation

Retention:

Permanent

\section{Scenarios Evaluation Tool for Chlorinated Solvent MNA}

(A Research Study of the Monitored Natural Attenuation/Enhanced Attenuation for Chlorinated Solvents Technology Alternative Project)

Contributing Researchers:

Michael J. Truex, Pacific Northwest National Laboratory

Charles J. Newell, Groundwater Services, Inc.

Brian B. Looney, Savannah River National Laboratory (SRNL)

Karen M. Vangelas, SRNL

Introduced by:

Karen Vangelas, SRNL

Brian B. Looney, SRNL

October 2, 2006

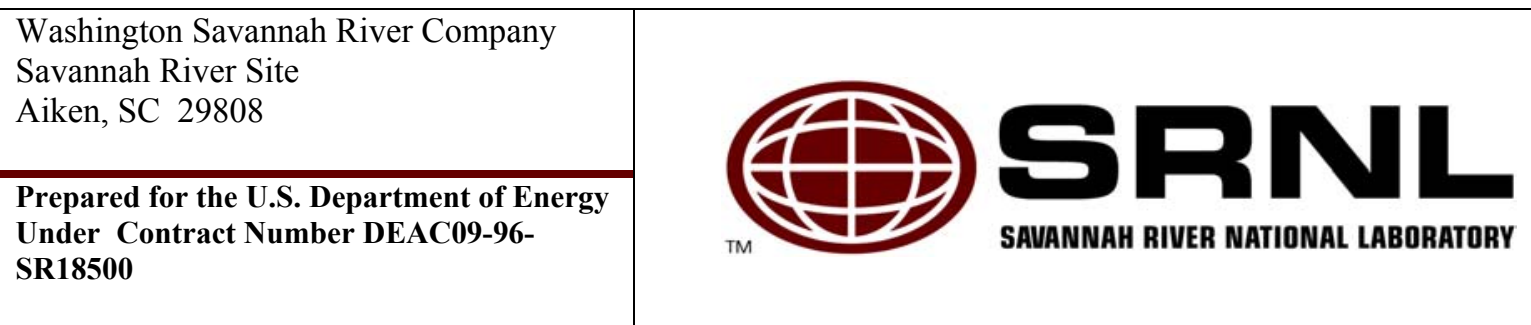




\section{Executive Summary}

One of the main challenges to implementing MNA is the need to cost-effectively interpret the multifaceted site specific data. To address this challenge, a team of researchers developed a "taxonomic key" to classify contaminated sites into one of thirteen scenarios based on hydrologic setting, geochemistry and a variety of modifying factors. The team developed a guidebook for each scenario to streamline characterization, modeling and monitoring. The result is a practical tool that will assist in environmental decision-making and in developing defensible environmental management strategies.

\section{Introduction}

Over the past three decades, much progress has been made in the remediation of chlorinated solvents from the subsurface. Yet these pervasive contaminants continue to present a significant challenge to the U.S. Department of Energy (DOE), other federal agencies, and other public and private organizations. The physical and chemical properties of chlorinated solvents make it difficult to rapidly reach the low concentrations typically set as regulatory limits. These technical challenges often result in high costs and long remediation time frames. In 2003,

the DOE through the Office of Environmental Management funded a science-based technical project that uses the U.S. Environmental Protection Agency's technical protocol (EPA, 1998) and directives (EPA, 1999) on Monitored Natural Attenuation (MNA) as the foundation on which to introduce supporting concepts and new scientific developments that will support remediation of chlorinated solvents based on natural attenuation processes. This project supports the direction in which many site owners want to move to complete the remediation of their site(s), that being to complete the active treatment portion of the remedial effort and transition into MNA.

The overarching objective of the effort was to examine environmental remedies that are based on natural processes - remedies such as Monitored Natural Attenuation (MNA) or Enhanced Attenuation (EA). The research program did identify several specific opportunities for advances based on: 1) mass balance as the central framework for attenuation based remedies, 2) scientific advancements and achievements during the past ten years, 3) regulatory and policy development and real-world experience using MNA, and 4) exploration of various ideas for integrating attenuation remedies into a systematic set of "combined remedies" for contaminated sites. These opportunities are summarized herein and are addressed in more detail in referenced project documents and journal articles, as well as in the technical and regulatory documents being developed within the ITRC.

Three topic areas were identified for development during this project. These areas are: mass balance, Enhanced Attenuation (EA), and new characterization and monitoring tools and approaches to support MNA and EA. Each of these topics is documented in stand alone reports, WSRC-STI-2006-00082, WSRC-STI-2006-00083, and WSRC-STI2006-00084, respectively. In brief, the mass balance efforts are examining methods and 
tools to allow a site to be evaluated in terms of a system where the inputs and processes within the system are compared to the outputs from the system, as well as understanding what attenuation processes may be occurring and how likely they are to occur within a system. Enhanced Attenuation is a new concept that is a transition step between primary treatments and MNA, when the natural attenuation processes are not sufficient to allow direct transition from the primary treatment to MNA. EA technologies are designed to either boost the level of the natural attenuation processes or decrease the loading of contaminants to the system for a period of time sufficient to allow the remedial goals to be met over the long-term. For characterization and monitoring, a phased approach based on documenting the site specific mass balance was developed. Tools and techniques to support the approach included direct measures of the biological processes and various tools to support cost-effective long-term monitoring of systems where the natural attenuation processes are the main treatment remedies. The effort revealed opportunities for integrating attenuation mechanisms into a systematic set of "combined remedies" for contaminated sites.

An important portion of this project was a suite of 14 research studies that supported the development of the three topic areas. A research study could support one or more of these three topic areas, with one area identified as the primary target. The following report documents the results of the development of a scenario-based framework to support MNA and EA decision-making led by Charles J. Newell of Groundwater Services Inc. and Michael Truex of Pacific Northwest National Laboratory. This study supports the topic area(s) of characterization and monitoring and Enhanced Attenuation with characterization and monitoring being the primary development area. The objective of the study was to develop a guide to provide practitioners with an appropriate level of site specificity to assist in planning/supporting characterization, modeling, and implementation of MNA and EA. The tool consists of a user's guide and 13 scenarios that are built around general site conditions and hydrogeologic conditions.

The Scenarios document is practical in its focus and scope but the investigators did an excellent job of weaving in the latest science (in the form of reaction mechanisms and rates) and in leveraging related efforts funded by DOD and EPA (e.g., BIOCHLOR, BIOPLUME, MAROS, etc.). This work builds significantly on the 1998 EPA protocol. In many cases, the historical datasets developed for these other projects were used as the basis for setting the boundaries on the bins (e.g., for flow rate changes, etc.). The idea of a taxonomic key for chlorinated organic MNA was a substantive challenge and the result is impressive. Anytime that a system is set up to organize and simplify a problem, there will be potential technical challenges, but this work is structured to encourage collection of key site specific data when those pitfalls are approached at any particular plume or plume zone.

The research team did a very good job of describing the various key concepts that a site owner needs to understand and communicate with respect to the viability of MNA. This product provides basic guidance on the different degradation mechanisms that are likely to occur given different site conditions. These scenarios should be beneficial to the user in focusing on key concepts/questions that pertain to their site conditions. 


\section{References for Introduction}

Page vi of vi

EPA, 1998. Technical Protocol for Evaluating Natural Attenuation of Chlorinated Solvents in Groundwater, EPA/600/R-98/128. Washington DC. September 1998.

EPA, 1999. Use of Monitored Natural Attenuation at Superfund, RCRA Corrective Action and Underground Storage Tank Sites, OSWER Directive 9200.4-17P. Washington DC. April 21, 1999.

WSRC-STI-2006-00082, 2006. Mass Balance: A Key to Advancing Monitored and Enhanced Attenuation for Chlorinated Solvents. Washington Savannah River Company, Aiken, SC, 29808. June 2006. Available at www.osti.gov.

WSRC-STI-2006-00083, 2006. Enhanced Attenuation: A Reference Guide on Approaches to Increase the Natural Treatment Capacity of a System, Revision 1. Washington Savannah River Company, Aiken, SC, 29808. August 2006. Available at www.osti.gov.

WSRC-STI-2006-00084, 2006. Characterization and Monitoring of Natural Attenuation of Chlorinated Solvents in Ground Water: A Systems Approach, Revision 1. Washington Savannah River Company, Aiken, SC, 29808. August 2006. Available at www.osti.gov. 


\section{Scenarios Evaluation Tool}

\section{for Chlorinated Solvent MNA}
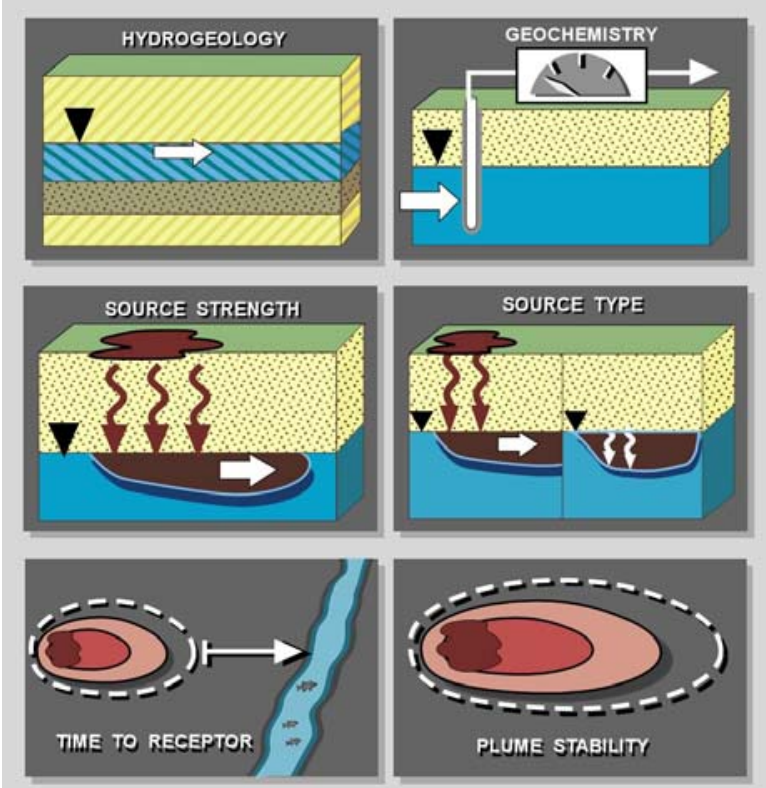

Michael J. Truex • Pacific Northwest National Laboratory Charles J. Newell - Groundwater Services, Inc.

Brian Looney - Savannah River National Laboratory Karen Vangelas - Savannah River National Laboratory

Produced as part of the DOE-sponsored Monitored Natural Attenuation Project coordinated by Savannah River National Laboratory 
THIS DOCUMENT IS A PRODUCT OF the Monitored Natural Attenuation / Enhanced Attenuation for Chlorinated Solvents Technology Alternative Project. The document was sponsored by the U.S. Department of Energy (DOE) Office of Cleanup Technologies and administered by the U.S. Department of Energy Savannah River (SR) Operations Office (Contract No. DE-AC0996SR18500). We appreciate the guidance and support of Claire H. Sink of DOE Headquarters and Karen M. Adams of DOE SR. The Savannah River National Laboratory provided technical direction for this project, as well as daily operations and management. We acknowledge the participation and collaboration of other federal agencies, notably, the U.S. Geological Survey (USGS) and the U.S. Environmental Protection Agency (EPA). 


\section{TABLE OF CONTENTS}

1.0 WHY SCENARIOS? 1

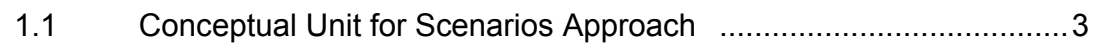

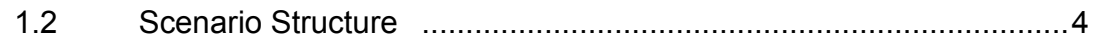

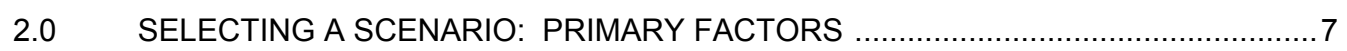

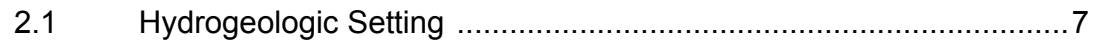

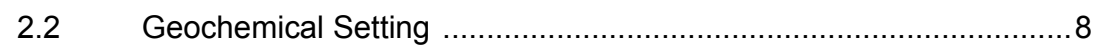

3.0 SELECTING A SCENARIO: MODIFYING FACTORS .................................... 10

3.1 Modifying Factor 1: Source Strength ................................... 10

3.2 Modifying Factor 2: Source Type ….................................... 12

3.3 Modifying Factor 3: Location of Receptors/Travel Time ...............12

3.4 Modifying Factor 4: Plume Stability_........................................ 13

3.5 Summary of Modifying Factors and Data Needs ......................... 15

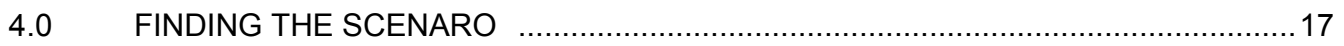

$5.0 \quad$ WHAT YOU CAN LEARN FROM EACH SCENARIO ....................................... 19

$5.1 \quad$ Dechlorination Reactions ................................................ 19

Key Processes: Potential for MNA Processes

5.3 Key Sustainability Concept ................................................ 35

5.4 Actions Needed to Determine MNA Viability ................................ 38

$5.5 \quad$ Key Monitoring Concepts …............................................. 41

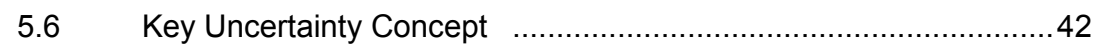

$5.7 \quad$ Key Data Analysis .............................................................. 43

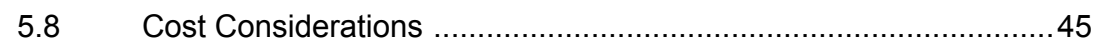

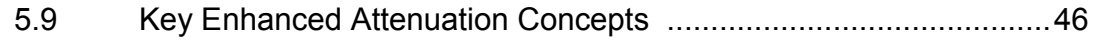

5.10 Key Source Control Concept ....................................................46 


\section{LIST OF TABLES}

TABLE 1. TABLE 2.

TABLE 3.

TABLE 4.

TABLE 5.

TABLE 6.

TABLE 7.

TABLE 8.

TABLE 9.

TABLE 10.

FIGURE 1. FIGURE 2. FIGURE 3. FIGURE 4.

FIGURE 5. FIGURE 6. FIGURE 7. FIGURE 8. FIGURE 9. FIGURE 10. FIGURE 11. FIGURE 12. FIGURE 13. FIGURE 14. FIGURE 15. FIGURE 16. FIGURE 17. FIGURE 18. FIGURE 19. FIGURE 20.

FIGURE 21.

FIGURE 22.

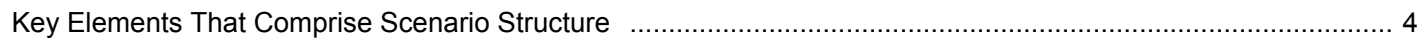

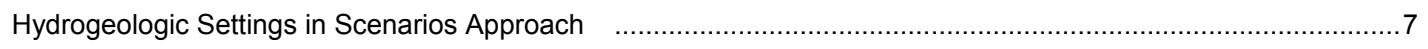

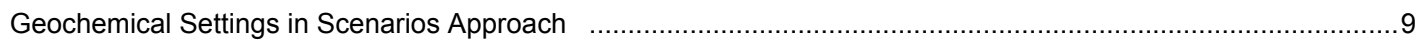

Summary of Modifying Factors and Data Required to Evaluate Modifying Factors. $\quad$......................................16

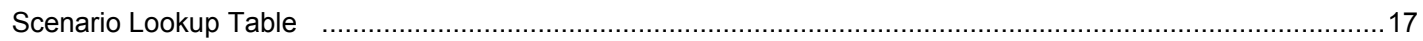

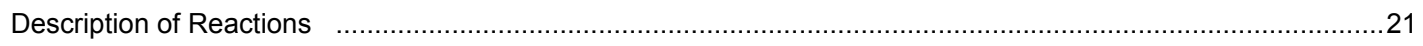

Additional Characterization Information to Assess Whether a Reaction Will Occur …....................................22

Sustainability of Attenuation Processes

Considerations for Selecting Modeling Approach Based on Site Properties ...................................................39

Relation of Time to Receptor and Monitoring System Category ................................................................42

\section{LIST OF FIGURES}

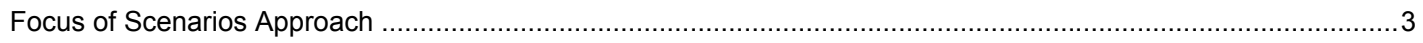

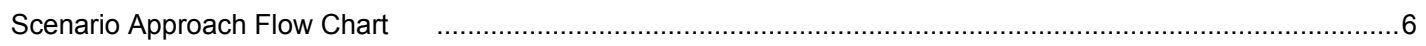

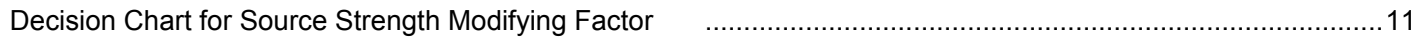

Three Source Types: Vadose Zone Only, Submerged Only, and Mixed Vadose Zone/Submerged.

Schematic of Distance from Edge of Plume Segment to Potential Receptor $\quad$...................................................12

Application of Mann-Kendall Statistics to Determine Plume Stability Category ................................................14

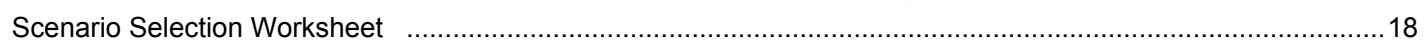

Dechlorination Reactions for PCE Under the Aerobic Geochemical Setting . .....................................................22

Dechlorination Reactions for PCE Under the Anoxic Geochemical Setting $\quad$......................................................24

Dechlorination Reactions for PCE Under the Anaerobic Geochemical Setting. . .................................................25

Dechlorination Reactions for 1,1,2,2-TeCA Under the Aerobic Geochemical Setting. .......................................26

Dechlorination Reactions for 1,1,2,2-TeCA Under the Anoxic Geochemical Setting. .....................................27

Dechlorination Reactions for 1,1,2,2-TeCA Under the Anaerobic Geochemical Setting ................................28

Dechlorination Reactions for 1,1,1,2-TeCA Under the Aerobic Geochemical Setting .........................................29

Dechlorination Reactions for 1,1,1,2-TeCA Under the Anoxic Geochemical Setting. . .......................................30

Dechlorination Reactions for 1,1,1,2-TeCA Under the Anaerobic Geochemical Setting. ...................................31

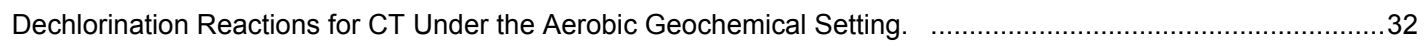

Dechlorination Reactions for CT Under the Anoxic Geochemical Setting ...................................................3

Dechlorination Reactions for CT Under the Anaerobic Geochemical Setting. .................................................34

Framework for Sustainability of Anaerobic Reactions at Chlorinated Solvent Sites

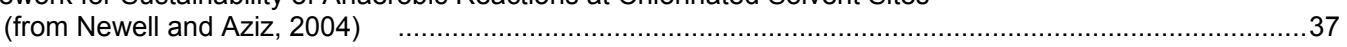

Monitoring Intensity Chart Based on Plume Stability Analysis for Wells in or Near the Subsurface Source ("Source") and Wells in Plume Segment Downgradient of Source ("Tail") ...................41

Two types of MNA Monitoring.

\section{APPENDICES}

APPENDIX 1

Selecting Hydrogeologic Setting from DRASTIC Settings 
WSRC-STI-2006-00096, Rev. 1

October 2, 2006

Page iv of iv

\section{SCENARIOS}

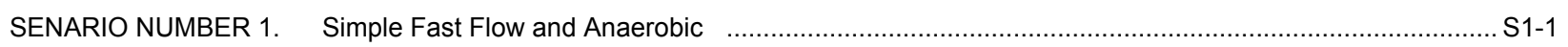

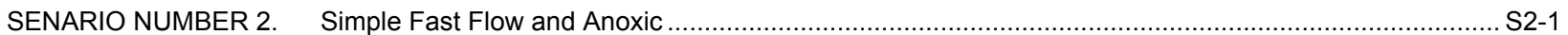

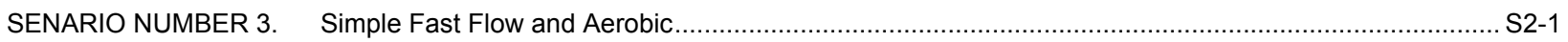

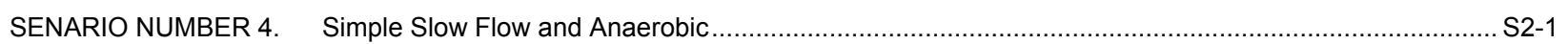

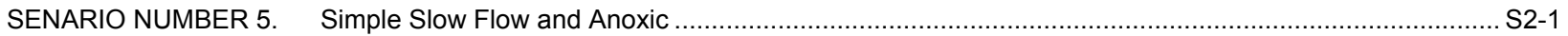

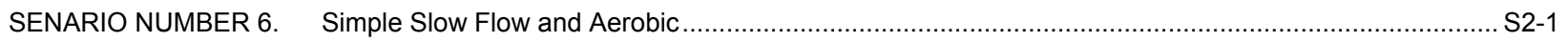

SENARIO NUMBER 7. Faster Flow with Significant Heterogeneities and Anaerobic ........................................................ S2-1

SENARIO NUMBER 8. Faster Flow with Significant Heterogeneities and Anoxic.......................................................... S2-1

SENARIO NUMBER 9. Faster Flow with Significant Heterogeneities and Aerobic ....................................................... S2-1

SENARIO NUMBER 10. Slower Flow with Significant Heterogeneities and Anaerobic ................................................... S2-1

SENARIO NUMBER 11. Slower Flow with Significant Heterogeneities and Anoxic .......................................................... S2-1

SENARIO NUMBER 12. Slower Flow with Significant Heterogeneities and Aerobic .......................................................... S2-1

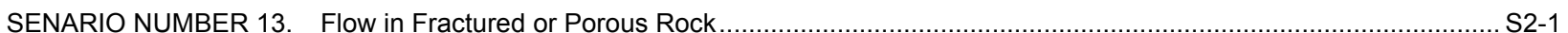




\subsection{WHY SCENARIOS?}

The 1998 EPA Technical Protocol for Evaluating Natural Attenuation of Chlorinated Solvents in Groundwater and other technical protocols describe the technical basis for evaluating Monitored Natural Attenuation (MNA) as a remedy. While MNA has been used successfully at hundreds of sites around the country (see text box, next page), most of these protocols have the following limitations:

- practitioners must decide on the level of detail and the information/analysis that is important to their specific site;

- given the wide range of source, hydrogeologic, geochemical, and degradation processes that affect MNA studies, the application of the protocol can be complex;

- existing classification schemes (such as Type 1, Type 2, Type 3 sites) are too limited;

- many of the protocols are designed for sites where anaerobic biodegradation is the dominate natural attenuation mechanism, while natural attenuation at other types of sites are not addressed directly;

- key concepts related to natural attenuation can be difficult to communicate to stakeholders (NRC, 2000).

This document uses a new approach to selecting or tracking the progress of MNA at chlorinated solvent sites. The approach presents a framework that links the MNA evaluation and associated decision logic to key site characteristics and known natural attenuation phenomena.

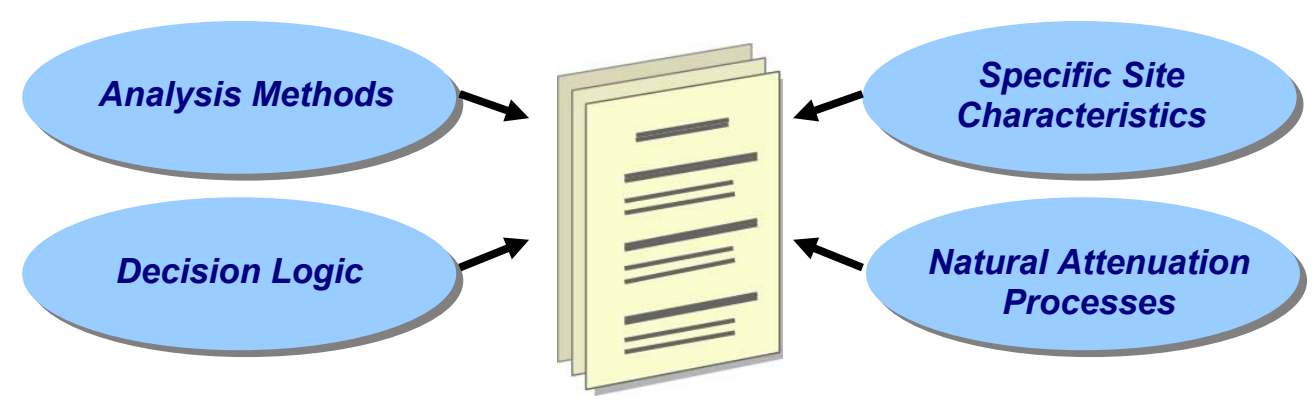

The approach is to take the wide spectrum of chlorinated solvent sites (e.g., different sources, hydrogeology, geochemistry, degradation process) and sort them into one of 13 different MNA scenarios. By applying a taxonomic system, the scenario that best describes a plume (or a segment of a plume) can be selected. The scenario contains information about how to proceed with MNA evaluation for the type of plumes that fit within the scenario.

The document is designed for site managers, technical personnel, consultants, and community representatives. Technically-oriented users will benefit from the concept of a new classification system for chlorinated solvent sites. Less technical users will benefit from the simplified structure of each scenario, where the key concepts and processes are highlighted.

Together site stakeholders will be able to understand, communicate, and make decisions about MNA in an accelerated and more efficient manner. Scenarios can be used in conjunction with other MNA protocols to assess the applicability of MNA at a particular site.

\section{KEY POINTS:}

Existing MNA protocols can be too focused on a particular type of site or attenuation process.

This guide provides a framework where the MNA methods and decision logic are linked together in one of 13 different "scenarios" or site types.

It is designed to be used together with more detailed protocols to understand MNA. 


\section{Will MNA Work? A Detailed Historical Analysis of 178 Sites}

A retrospective "Historical Analysis of MNA" survey was developed to gain a better understanding of the application of MNA at sites affected with chlorinated solvents. The survey sought to provide insights into the remediation professional's general experience with MNA and to gather site-specific data regarding the implementation of MNA as a remedy of a particular CVOC plume. The survey was distributed to approximately 230 remediation professionals from industry, government, and academia with experience in the field of MNA.

The survey was divided into two parts: i) general request for information about MNA experiences and ii) more detailed data on a specific site. Survey data was received from 30 individuals for a total of 178 waste sites; all respondents provided Part A data, and site-specific data was received for 42 individual chlorinated solvent plumes. Data from Part A and Part B are summarized in and interpreted in a report and technical paper (McGuire et al, 2003 and McGuire et al, 2004) that contains the full text and interpretation from this line of inquiry.

Overall the study indicated that MNA had been used extensively at chlorinated solvent sites. Detailed conclusions from the study are summarized below:

\section{GENERAL SURVEY}

- $\quad$ MNA was determined to be feasible as a remedy at over $75 \%$ of the sites where the application of MNA was evaluated ( $36 \%$ sole remedy, $46 \%$ with other treatment).

- Importantly, MNA was determined to be infeasible at about $23 \%$ of the sites.

- At sites where MNA is used with an active treatment, the active treatment is still in operation at approximately $72 \%$ of those sites.

- $\quad$ The average cost of the entire initial MNA study was reported to be about $\$ 188,000$ and results ranged from $\$ 10,000$ to $\$ 750,000$.

- The average annual cost for monitoring an MNA site was found to be $\$ 32,000$ with a range of $\$ 3,000$ to $\$ 150,000$.

- Nearly half of the respondents reported that the typical size of a chlorinated solvent plume where MNA is utilized in the remedial scheme is 10 to 50 acres, while $29 \%$ and $25 \%$ reported the average size to be less than 10 acres and greater than 50 acres, respectively.

\section{TECHNICAL SURVEY}

- $\quad$ MNA is used as a remedy at a variety of industrial sites with a broad range of processes.

- $\quad$ The 1998 EPA protocol (EPA, 1998 and 1999) was most often referenced as the guideline for MNA implementation (36\%). Notably, almost $29 \%$ used a site specific protocol. Other protocols used as the basis for the reported sites included:

○ $12 \%$ state protocol, $19 \%$ other, and $5 \%$ National Research Council (NRC) MNA review (NRC, 2000).

- Almost $70 \%$ of respondents stated that anaerobic degradation is the primary natural attenuation process occurring in the plume, while the remaining attenuation processes each accounted for less than $7 \%$

- A variety of geochemical indicators are reportedly used to assess MNA, but over $90 \%$ rely on the presence of biodegradation daughter products.

- A variety of tools were used to support MNA, including conceptual models, analytical models, and mass flux calculations. About $19 \%$ of the respondents reported that none of these approaches was used in implementing MNA.

- $\quad$ Computer models of various types were used to evaluate MNA at $57 \%$ of the sites. The most common model used was BIOCHLOR.

In summary, the MNA historical analysis showed that MNA had been evaluated, attempted, or applied at a large number of chlorinated solvent sites. This widespread use of MNA for a variety of plume types, contaminants, and hydrogeologic settings supports the premise that a Scenarios Based Approach can provide a useful framework for evaluating MNA. 


\subsection{Conceptual Unit for Scenarios Approach}

For the purpose of the scenarios approach, we have divided a groundwater contamination project at a particular site into the following components:

- Entire Site: all of the components listed below;

- Surface Source(s): the point on the surface where contaminants entered the subsurface;

- Subsurface Source(s): source materials below the surface, such as contaminated soils, NAPLs, sorbed contaminants, and contaminants dissolved in the matrix;

- Plume System: a single hydraulically connected plume that emanates from one or more subsurface sources, but is separated from other plumes at the site geographically (in a different spatial location at the site) and/or geologically (in a different hydrogeologic unit at the site);

- Plume Segment(s): a geographic subarea of a plume system where the hydrogeologic, contaminant distribution, and geochemical conditions are similar, and where the same degradation processes are active throughout the subarea;

- Receptor(s): the human and/or environmental receptors that are or could be affected by the plume. Note that any plume segment could have a receptor (for example, a near source plume segment could have indoor air receptors), and that some plume segments (including end segments) may not have any receptors.

A schematic of this nomenclature is shown below In Figure 1. In this example, a surface source creates a subsurface source, which then produces a plume system. Each plume system is further divided into several plume segments as needed where the contaminant distribution, hydrogeology and geochemistry are similar. For example, the first plume segment might be anaerobic near the source followed by an aerobic plume segment.

Note you can make a site as simple (one plume segment) or as complex (many plume segments) as you want.

\section{KEY POINTS:}

To use this document, you must divide your site up into plume segments where the hydrogeologic, contaminant distribution, and geochemical conditions are similar.

Some sites will only have one plume segment. Others will have several. Upgradient plume segments will serve as the source of the contaminant loading to a downgradient segment.

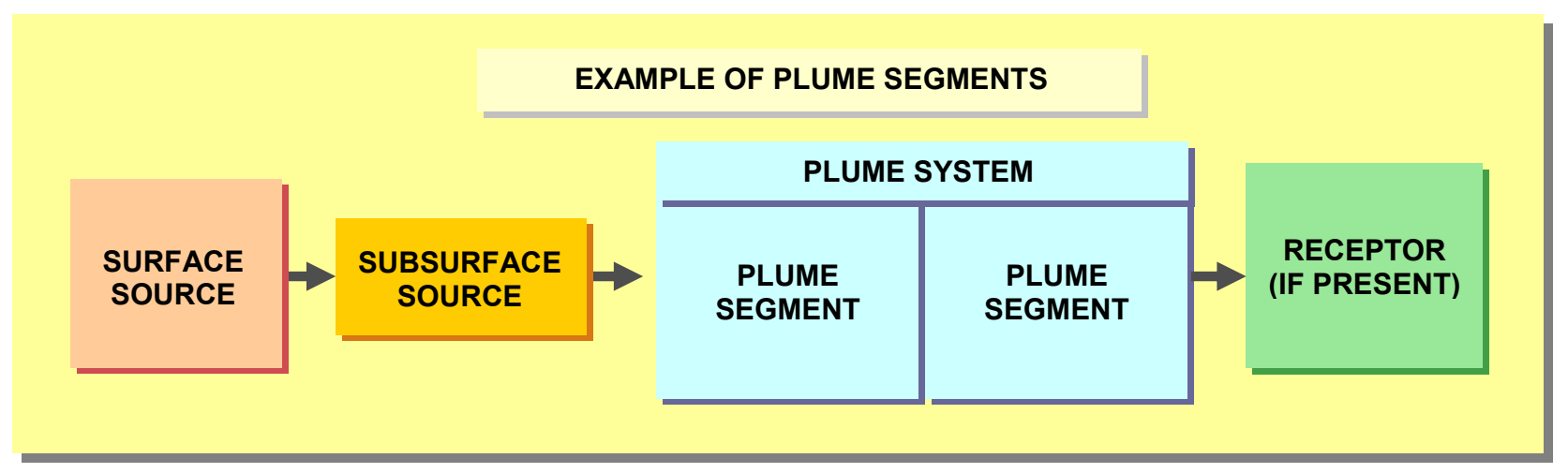

FIGURE 1. Focus of Scenarios Approach: Plume Segments. For each plume segment, use the flowchart on page 6 to select which scenario best represents a plume segment. 


\subsection{Scenario Structure}

A scenario is a particular combination of five different generic hydrogeologic settings and three geochemical environments (see Table 1) and each Scenario includes up to four modifying factors:

TABLE 1. Key Elements That Comprise Scenario Structure

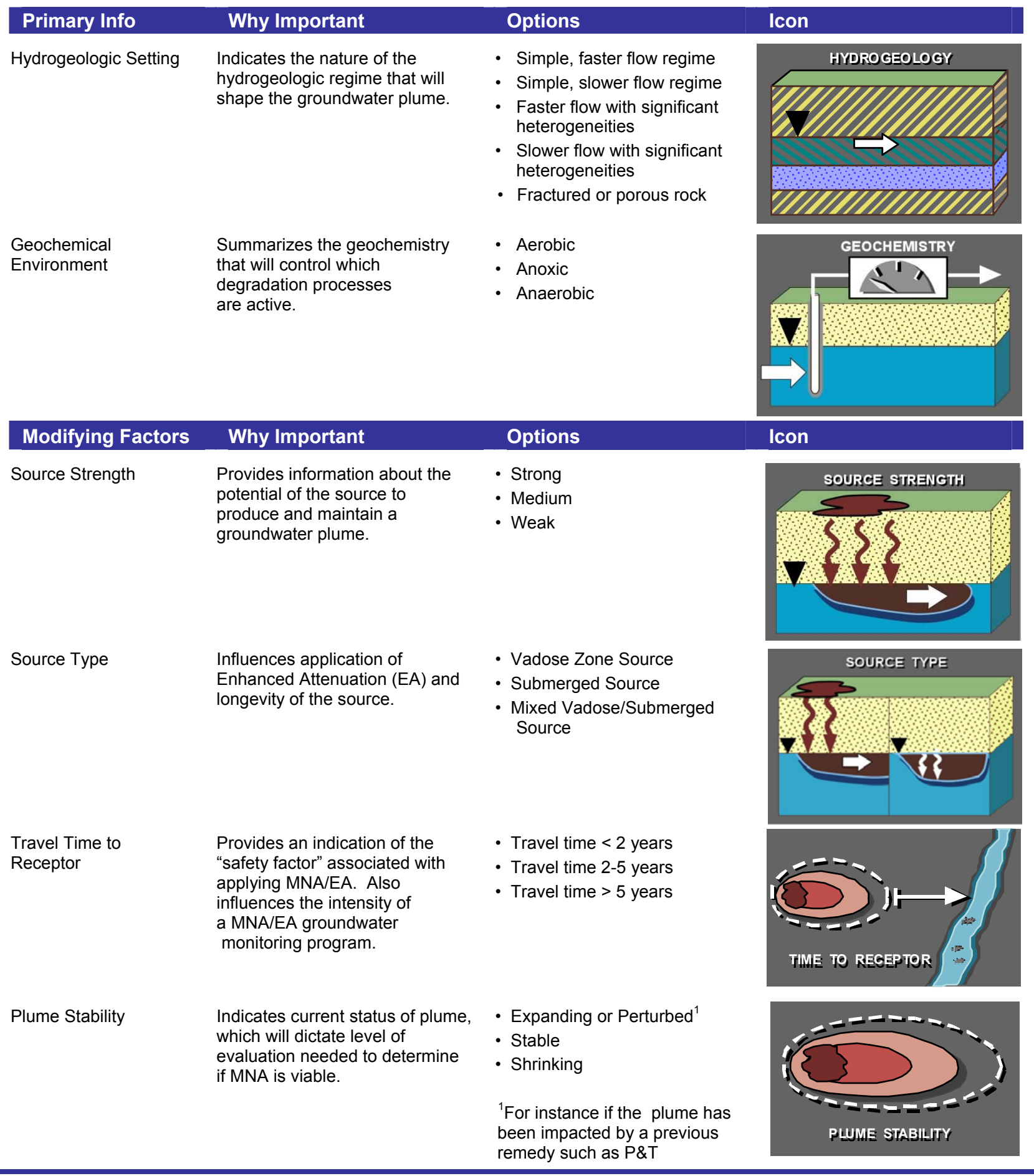


Once you have a selected a scenario, you can go to the particular scenarios page and get the following information:

- SCENARIO DESCRIPTION

- Hydrogeologic Setting

- Geochemical Setting

- KEY DECHLORINATION REACTIONS

- EFFECT OF MODIFYING FACTORS

- WILL MNA WORK?

- Potential for MNA Processes to Control Plume

- Key Sustainability Concept

- HOW DO I CHARACTERIZE THIS TYPE OF SITE?

- Actions Needed to Determine MNA Viability

- Key Monitoring Concepts

- Key Uncertainty Concepts

- HOW DO I ANALYZE DATA?

- WHAT ABOUT COSTS AND ENHANCEMENTS?

- Cost Considerations

- Key Enhanced Attenuation Concepts

- Key Source Control Concepts

In summary, you pick a scenario that best matches your plume segment (1 or more) using the methods described in Section 2, below. After you pick the scenario, you go to the scenario summary page (listed in the lookup Table 5) to learn more about how monitored natural attenuation fits into a plume management strategy for that scenario. Figure 2 shows an outline of the overall process.

The advantages of the scenario approach are:

- Plume segments are classified into 13 different categories, each with "segment-specific" information about processes, characterization, data analysis, and other factors;

- Key information about processes are summarized in a concise, distilled manner;

- The scenario allows stakeholders from a variety of backgrounds and levels of technical expertise to focus on a few key concepts, helping communication and overall understanding of the MNA issues at the site. 


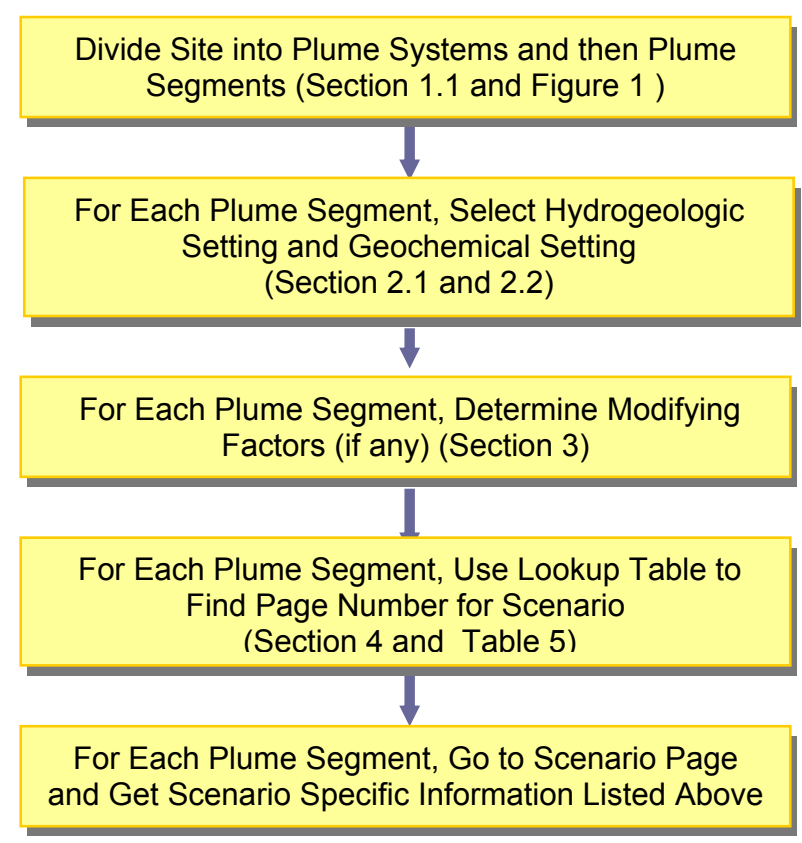

FIGURE 2. Scenario Approach Flow Chart

\section{KEY POINTS:}

Scenarios are based on Primary Information (hydrogeology and geochemistry) and Modifying Factors (related to the source and risk elements).

Once the scenario is selected, you go to the scenario summary page for that scenario and learn about monitoring, key processes, data analysis, cost, and other issues specific to that scenario 


\subsection{SELECTING A SCENARIO: PRIMARY FACTORS}

\subsection{Hydrogeologic Setting}

Hydrogeology influences a number of key natural attenuation processes, such as 1) the mass flux leaving a source zone; 2 ) the time that is available for degradation to occur while contaminants are migrating in groundwater; 3) the source duration; 4) what type of groundwater monitoring system will be required (for example, a simple system vs. complex system); and 5) how amendable the site will be for Enhanced Attenuation (EA).

\section{How to Pick a Hydrogeologic Setting}

The scenarios approach requires that one of the following five hydrogeologic settings must be selected for your plume segment (Table 2):

TABLE 2. Hydrogeologic Settings in Scenarios Approach

\begin{tabular}{ll} 
Hydrogeologic Setting & Description \\
\hline H1. Simple, faster flow regime & $\begin{array}{l}\text { Sandy or gravelly aquifers where the plume is primarily in one } \\
\text { hydrologic unit (simple geology) }\end{array}$ \\
\hline H2. Simple, slower flow regime & $\begin{array}{l}\text { Silty or silty sand aquifers where the plume is primarily in one } \\
\text { hydrologic unit (simple geology) }\end{array}$ \\
\hline H3. Faster flow with significant heterogeneities & $\begin{array}{l}\text { Layers of sand or gravel and aquitards of silt or clay/outwash and till } \\
\text { geology (alluvial, glacial, river basin) }\end{array}$ \\
\hline H4. Slower flow with significant heterogeneities & $\begin{array}{l}\text { Layers of silt or silty sand and aquitards of silt or clay/till geology } \\
\text { (alluvial, glacial, river basin) }\end{array}$ \\
\hline H5. Fractured or porous rock & \begin{tabular}{l} 
Plumes where the primary migration is through consolidated material \\
\hline
\end{tabular}
\end{tabular}

For some plume segments it will be obvious which of the five hydrogeologic settings provides the best match to actual hydrogeologic conditions in the plume segment. The following rules-of-thumb can also be used to help select the best hydrogeologic setting:

Rule-of-Thumb 1: The median groundwater seepage velocity from a survey of 400 contaminant sites around the country was $88 \mathrm{ft} / \mathrm{yr}$ (Newell et al., 1990). An unconsolidated site significantly faster than $88 \mathrm{ft} / \mathrm{yr}$ would likely be classified as one of the "faster" flow regimes (hydrogeologic settings $\mathrm{H} 1$ or $\mathrm{H} 3$ ), while a site slower than $88 \mathrm{ft} / \mathrm{yr}$ would likely be classified as one of the "slower" flow regimes (hydrogeologic settings $\mathrm{H} 2$ or $\mathrm{H} 4$ ). If your site is near the median value of $88 \mathrm{ft} / \mathrm{yr}$, you can use Rule-of-Thumb 2 (below), or use the "faster" classification to be conservative (e.g., to underpredict the ability of natural attenuation processes to control a plume).

Rule-of-Thumb 2: Sites where the predominate aquifer material is classified as GW, GP, GM, GC, SW, SP using the Unified Soil Classification System would be defined as "sandy or gravelly aquifers" (hydrogeologic settings $\mathrm{H} 1$ or $\mathrm{H} 3$ ). Sites where the predominate aquifer material is classified as SM, SC, ML, CL, OL, MH, or $\mathrm{CH}$ would be classified as having silt or silty sand or clay/till geology (hydrogeologic settings $\mathrm{H} 2$ or $\mathrm{H} 4$ ). 
Detailed Method: A more detailed method for evaluating which hydrogeologic setting best matches your plume segment is based on the U.S. EPA's DRASTIC system (Allen et al., 1987). DRASTIC includes a description of 88 hydrogeologic settings that can also be used as a resource for selecting a scenario. Appendix 1 contains a decision tree to select one of the 88 different DRASTIC settings, and shows which of the five hydrogeologic settings for this scenarios document best match each DRASTIC setting (see Appendix 1).

\subsection{Geochemical Setting}

The geochemical setting drives the types of degradation reactions that are present in a particular plume segment. As discussed in each scenario, it is important to understand the natural attenuation processes to manage the plume using MNA. The geochemical setting is also important for assessing the type of enhancement that may be required when degradation reactions are not sufficient under natural conditions. Section 5 contains additional information on the specific types of degradation reactions that can be expected to occur under each geochemical condition.

\section{How to Pick A Geochemical Setting}

To apply the scenarios approach, one of the following three geochemical environments must be selected for your plume segment using the following simple rules (Table 3): 
WSRC-STI-2006-00096, Rev. 1

October 2, 2006

Page 9 of 50

TABLE 3. Geochemical Settings in Scenarios Approach

\section{Geochemical Environment}

G1. Anaerobic

\section{Description (see note below about use of these values)}

Average dissolved oxygen concentration $<\sim 1 \mathrm{mg} / \mathrm{L}$ (if meter) or $<\sim 0.5 \mathrm{mg} / \mathrm{L}$ (if test kit);

AND

Sulfate concentration < $50 \mathrm{mg} / \mathrm{L} ; \quad$ (value applies to most but not all sites) AND

Nitrate < 1 mg/L;

AND

Methane OR ferrous iron OR sulfide must be detected in most of the wells; AND

TOC > $5 \mathrm{mg} / \mathrm{L}$;

AND

Dechlorination products must be present in the plume

G2. Anoxic

Average dissolved oxygen concentration $<\sim 2 \mathrm{mg} / \mathrm{L}$ (by meter or by test kit); AND

Plume doesn't meet all of the anaerobic indicators

Average dissolved oxygen concentration $>\sim 2 \mathrm{mg} / \mathrm{L}$ (by meter or by test kit);

AND

Plume doesn't meet ANY of the anaerobic indicators

With these criteria, a plume can be classified as Anaerobic, Anoxic, or Aerobic.

NOTE: All criteria listed for a geochemical category must generally be satisfied to be selected as the geochemical setting. The criteria statements and numeric values should not be used as absolute rules. Technical judgment and knowledge of site conditions should be applied in conjunction with these guidelines when determining the site geochemical setting.

\section{KEY POINT:}

Geochemistry is a primary factor and is used to determine if your plume segment is aerobic, anaerobic, or anoxic. 


\subsection{SELECTING A SCENARIO: MODIFYING FACTORS}

Each scenario contains information for different variations in the scenario based on modifying factors associated with source and plume characteristics. Modifying factors that can be defined and carried into each scenario include:

- $\quad$ Source Strength

- Source Type

- Location of Receptors/Travel Time

- Plume Stability

\subsection{Modifying Factor 1: Source Strength}

At actual sites, Source Strength is associated with the contaminant mass flux (in units of mass per time) leaving the source and the mass in the source (in mass units). Mass flux is the mass discharge rate leaving the source zone in units of mass per time. Mass flux is often estimated by calculating the groundwater Darcy velocity, multiplying velocity by the cross-sectional area of the downgradient projection of the source to get a groundwater flux, and multiplying this groundwater flux by a representative concentration for the source zone. An analytical or numerical model will also estimate mass flux in a similar way. The source mass is the reservoir of contaminants held in the source zone either as non-aqueous phase liquids; contaminants that have diffused into the matrix (e.g., fractured rock or clays), or adsorbed contaminants. Estimating source mass is difficult and order-of-magnitude results are typical at many sites. Use of the dissolved mass estimates for the total mass in the plume system will be inaccurate at most sites, as most of the contaminant mass resides in the source materials or the aquifer matrix.

In theory, source strength is a function of source mass and the mass flux leaving the source: Mass flux defines the contaminant loading to the plume that will need to be attenuated. At sites where there is a small mass flux from a large source, the source will exist for a long time without source reduction. Alternatively, at sites with a large mass flux from a small source, the source will be quickly depleted. Many sites fall in between these two conditions and it is important to quantify mass loading and assess whether source reduction is needed to help MNA meet remediation goals.

If source concentration data over a significant amount of time is available, the source longevity can be estimated by calculating a source decay rate constant $\left(\mathrm{k}_{\text {point }}\right)$ using the method of U.S. EPA (2002) to obtain order-of-magnitude estimates of remediation timeframe for MNA. Many years of data and a $90 \%$ reduction in concentration is often required to obtain reliable results, however (U.S. EPA, 1999).

To capture these important source characteristics, while at the same time recognizing that there may not be any information about the actual mass that may have been released, the following semiquantitative classification system was developed to define a Source Strength as either being Strong, Medium, or Weak (Figure 3): 


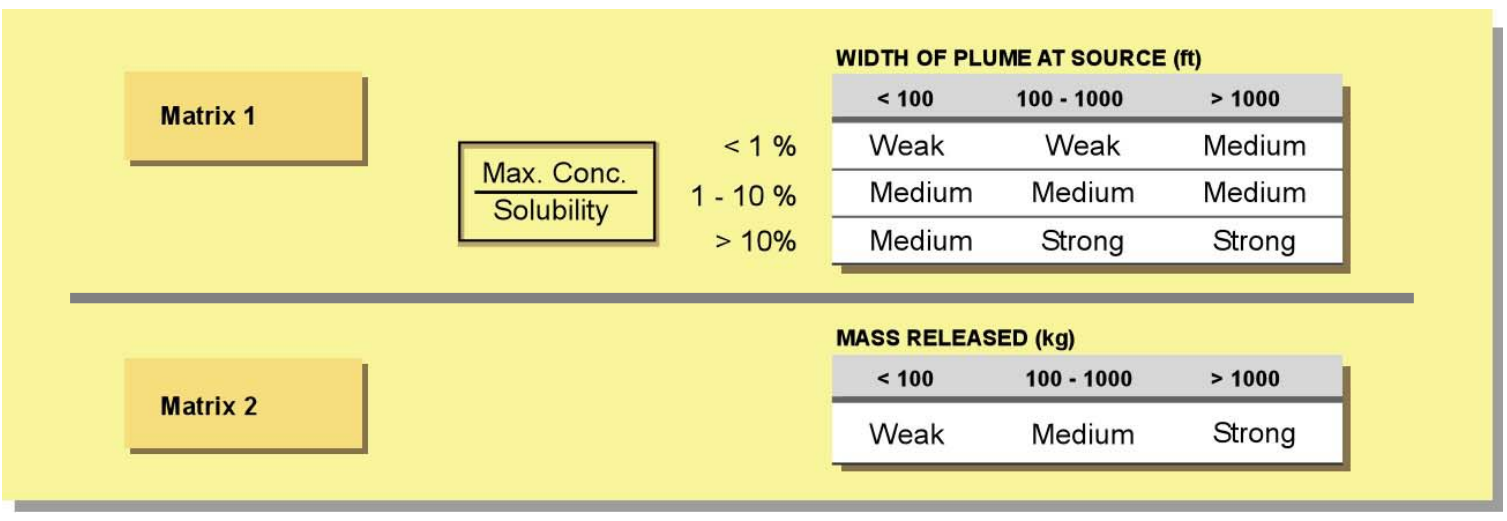

FIGURE 3. Decision Chart for Source Strength Modifying Factor

To use the classification system, use either Matrix 1 or Matrix 2 or both depending on available data (or the confidence in estimated values). Matrix 1 is used to distinguish between Strong, Medium, and Weak sources on the basis of three easily-obtained values: Width of the plume at the source (in feet); the maximum concentration of the key contaminant (in $\mathrm{mg} / \mathrm{L}$ ); and the pure-phase solubility of the key contaminant (in $\mathrm{mg} / \mathrm{L}$ ). After dividing the maximum concentration by the pure-phase solubility, Matrix 1 is applied.

If the original release mass of the key contaminant is known, the Source Strength from Matrix 1 can be modified using the value from Matrix 2, where the "strongest source" from either Matrix 1 or Matrix 2 is used. If the mass of the release is not known, the answer from Matrix 1 is used.

If the plume system has more than one plume segment, each downgradient plume segment will use the concentration and width data from the plume segment that is immediately upgradient for Matrix 1, if Matrix 1 is used.

\section{DNAPL AND MNA:}

Can MNA be used at a DNAPL site?

Note that the U.S EPA Directive (1999) states that:

"EPA expects that source control and long-term performance monitoring will be fundamental components of any MNA remedy."

MNA can play a significant role at sites with DNAPL. Natural attenuation processes occur at all sites, including those with DNAPL. These processes can: i) control the migration of the plume emanating from a DNAPL source zone, and ii) remove contaminants from the DNAPL source zone via dissolution.

If Matrix 2 is used, every plume segment will use the mass data from farthest upgradient plume segment (i.e., the plume segment that contains the source materials).

Note the two matrices were developed based on engineering judgment of the authors, and were not derived from detailed site databases, source characterization work, or modeling studies.

\subsection{Modifying Factor 2: Source Type}

The source type is an important modifying factor because it impacts source longevity and the ease of applying EA. Three different types of sources are defined (Figure 4):

- Vadose Zone Sources

- Submerged Sources

- Mixed Vadose/Submerged Sources 

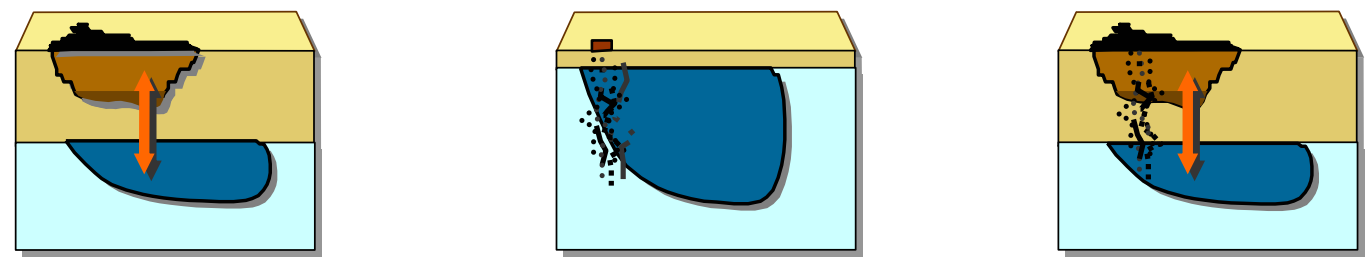

FIGURE 4. Three Source Types: Vadose Zone Only, Submerged Only, and Mixed Vadose Zone/Submerged.

The type of source is important for evaluating source strength, source longevity, and for treating the source. The longevity of a vadose zone source may be different than the longevity of a submerged source (e.g., see Johnson et al, 2003 for a comparison of vadose zone vs. submerged source zones at hydrocarbon sites). In fine-grained soils, the mass flux of contaminants from a vadose zone source may be small. Finally, vadose zone sources may be easier to treat with enhancements than submerged sources (e.g., through vapor extraction).

The primary source zones contributing to the groundwater plume must be identified and one of the three modifying factors must be selected. In general, sites with thicker vadose zones ( $>30$ feet) are more likely to have a vadose zone source. Sites with thinner vadose zones $(<20 \mathrm{ft})$ are more likely to have submerged sources. The material that comprises the vadose zone (clay vs. sand) will play an important role in controlling whether a site is dominated by a vadose zone source vs. a submerged source. Clay vadose zones will hold more source material in the vadose zone but have less infiltration, resulting in weak but long-lived sources. Sandy vadose zones may have a higher flux to groundwater over a shorter lifetime.

\subsection{Modifying Factor 3: Location of Receptors/Travel Time}

Receptors are either i) nearby groundwater wells that could capture plume contaminants or ii) the downgradient point where the groundwater plume discharges to a stream, or iii) an administrative boundary such as the property line or other regulatory-based location (Figure 5).

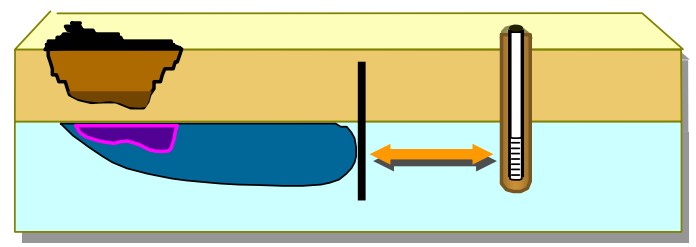

FIGURE 5. Schematic of Distance from Edge of Plume Segment to Potential Receptor

Travel time is an important factor in determining the intensity of the MNA/EA monitoring program. In addition, the travel time is also an important modifying factor because it indicates a general "margin of safety" in the case the MNA/EA fails and an alternative remedy must be implemented.

A physical receptor location (e.g., a well or stream discharge location) can be determined by water well surveys, evaluation of topographic maps, inspections of the land in the vicinity of the plume, and other methods. Administrative receptor locations will be determined by existing environmental regulations or negotiations with regulatory officials.

The travel time to the receptor is defined as to the distance to the receptor divided by the groundwater seepage velocity. For the scenarios approach, the travel time modifying factor is divided into three categories:

- Receptor is $<2$ years groundwater travel time.

- Receptor is between 2 and 5 years groundwater travel time

- Receptor is $>5$ years groundwater travel time 
These three categories are based on a system for evaluating initial site response developed as part of the ASTM Standard Guide for Risk-Based Corrective Action (ASTM, 2004).

Key concepts related to receptors/travel time are summarized below:

1. If groundwater seepage velocity is not known, one can assume a nominal groundwater/contaminant velocity of 88 feet per year ( $\sim 0.25 \mathrm{ft} /$ day) (Newell et al., 1999), so that the travel time (in days) is equal to the number of feet from the plume to the receptor location divided by 4 .

2. The simplest approach is to divide the distance from the downgradient edge of the plume to the receptor by the seepage velocity. Groundwater seepage velocity (also called groundwater linear velocity or interstitial velocity) is calculated using $V=K i / n$ where $V$ is the linear velocity, $K$ is the average hydraulic conductivity, $i$ is the hydraulic gradient, and $n$ is the effective porosity. This approach is based on describing groundwater flow by Darcy's Law.

3. The simple approaches in Step 1 or Step 2 can be modified to account for the effects of sorption to the aquifer matrix.

4 More sophisticated approaches (e.g., hydraulic relationships, computer modeling) may be needed to refine the results of the simple methods (Methods 1-3 above) to account for the effects of contaminant reaction and transport. Similarly, the effect of capture zones from pumping wells can be simulated using capture zone relationships and/or computer models.

5. An alternative method can be employed if a concentration vs. distance degradation rate for dissolved contaminants in a plume segment can be estimated (assuming no sorption or dispersion, only advection and degradation). The calculation equation is $C / C_{0}=\exp ^{-\lambda t}$, where $C / C_{0}$ is the fractional percentage of the original concentration, $t$ is the travel time in years away from the source and $\lambda$ is the degradation half-life in years. For example, if the travel time $t$ is four years, and the degradation rate $\lambda$ is 0.5 per year, then the concentration at the receptor will be about $14 \%$ of concentration at the edge of the segment $\left(0.14=\exp ^{-(0.5 \times 4)}\right.$. A degradation rate can be estimated from this technique if the concentration and travel time are known and if the plume is at steady-state at the upgradient and downgradient locations.

\subsection{Modifying Factor 4: Plume Stability}

Plume stability is a modifying factor because it dictates the level of evaluation needed to determine if MNA is sufficient. In the scenarios approach, we classify the Plume Stability modifying factor into one of the following categories:

- Expanding or Perturbed ${ }^{1}$

- Stable

- Shrinking

- No trend observable based on available data (in this case the plume stability modifying factor must be estimated by fate and transport analysis)

${ }^{1}$ For instance if the plume has been impacted by a previous remedy such as P\&T

Plume stability can be demonstrated by assessing standard groundwater contaminant concentration data over time with the following graphical techniques:

- Concentration vs. time plots at individual wells;

- Concentration vs. distance plots, with multiple lines for different sampling events through time;

- Plume maps of multiple plume extent at different times (i.e., either panel maps, or one map with several plume boundaries for different times). 
One method of assessing plume stability is based on determining the Mann-Kendall " $S$ " statistic, the confidence factor (CF), and the coefficient of variation (Aziz et al., 2003) (Figure 6) by:

- Using individual monitoring wells in the plume segment, and then determining the average trend over all the wells, or

- Performing the statistical analysis on a measurement of plume length.
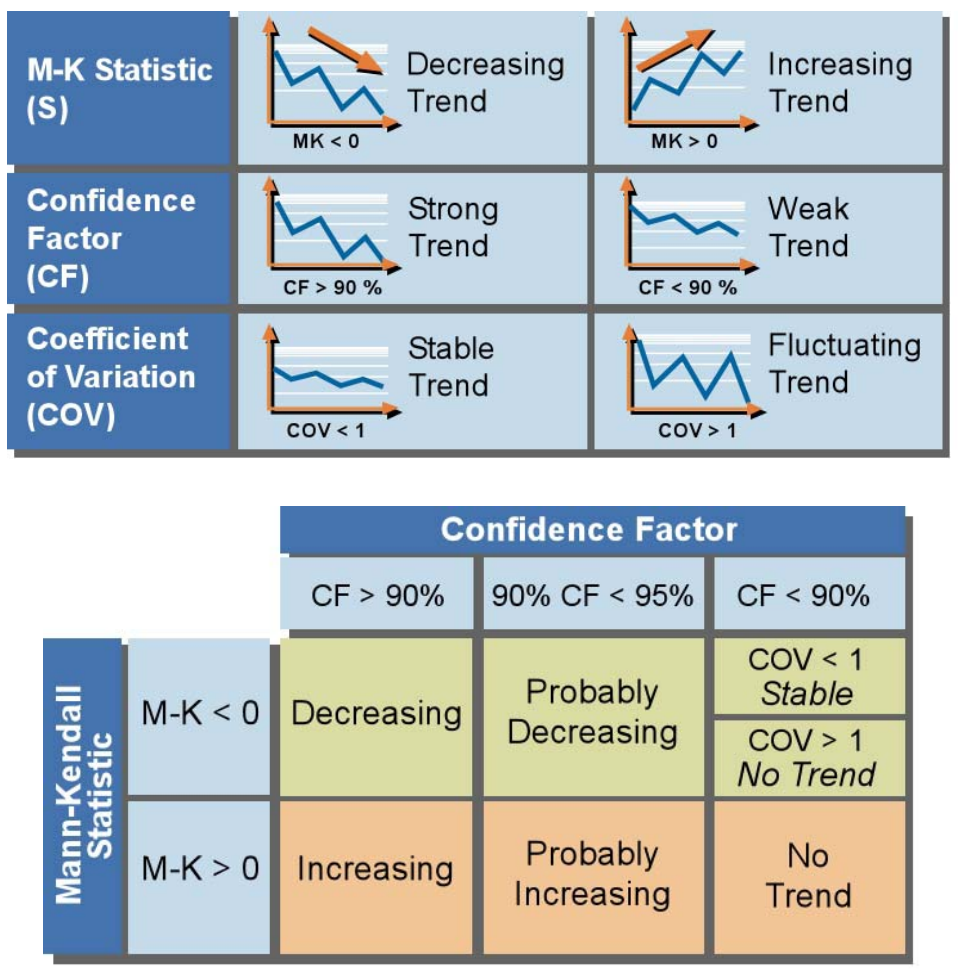

FIGURE 6. Application of Mann-Kendall Statistics to Determine Plume Stability Category

Other commonly used statistical approaches include the Mann-Whitney method, the MAROS software program designed to evaluate plume stability (Aziz et al., 2000b) (www.gsi-net.com), and the Natural Attenuation Software (NAS) system (Chapelle et al., 2003) where plume stability conditions over time can be evaluated.

Computer modeling can also be used as a supporting tool to evaluate plume stability. A computer model (one that accounts for plume change over time) is calibrated to existing data, then the model is used to predict plume behavior in the future. Commonly used models include BIOCHLOR (www.epa.gov/ada/csmos/models.html) and RT3D (http://bioprocess.pnl.gov/rt3d.htm).

\subsection{Summary of Modifying Factors and Data Needs}

The modifying factors are key site characteristics that augment the primary factors that define each scenario and increase the understanding of a site for evaluating and implementing MNA. As discussed above, some specific information about the site is needed to appropriately determine the modifying factors for a site. The modifying factors and related data needs are summarized in Table 4. 
TABLE 4. Summary of Modifying Factors and Data Required to Evaluate Modifying Factors.

\begin{tabular}{|c|c|c|}
\hline SOURCE STRENGTH & DATA NEEDS & GO TO SECTION: \\
\hline $\begin{array}{l}\text { Submerged Source } \\
\text { Qualitative Estimate } \\
\text { use Figure } 3\end{array}$ & $\begin{array}{l}\text { To use Figure 3, estimates are needed for the maximum concentration in } \\
\text { groundwater, solubility of contaminant(s), width of plume based on } \\
\text { groundwater data, estimate of source mass (typically from historical data). }\end{array}$ & 3.1 \\
\hline $\begin{array}{l}\text { Quantitative Estimate } \\
\text { Source Mass }\end{array}$ & $\begin{array}{l}\text { Historical site data, detailed site characterization (e.g., aquifer core } \\
\text { characterization, groundwater concentrations, DNAPL characterization } \\
\text { techniques). }\end{array}$ & 3.1 \\
\hline Mass Flux & $\begin{array}{l}\text { Groundwater concentrations in source area, hydraulic data to define Darcy } \\
\text { velocity, downgradient cross sectional area, specific flux measurement } \\
\text { techniques (e.g., integrated pump test, passive flux meter). }\end{array}$ & 3.1 \\
\hline $\begin{array}{l}\text { Vadose Zone Source } \\
\text { Qualitative Estimate } \\
\text { use Figure } 3\end{array}$ & $\begin{array}{l}\text { To use Figure 3, estimates are needed for the maximum concentration in } \\
\text { groundwater, solubility of contaminant(s), width of plume based on } \\
\text { groundwater data, estimate of source mass (typically from historical data). }\end{array}$ & 3.1 \\
\hline $\begin{array}{l}\text { Quantitative Estimate } \\
\text { Source Mass }\end{array}$ & $\begin{array}{l}\text { Historical site data, detailed site characterization (e.g., aquifer core } \\
\text { characterization, groundwater concentrations, soil gas concentrations, DNAPL } \\
\text { characterization techniques). }\end{array}$ & 3.1 \\
\hline Mass Flux & $\begin{array}{l}\text { Groundwater concentration in groundwater under the source area, hydraulic } \\
\text { data to define Darcy velocity, downgradient cross sectional area, specific flux } \\
\text { measurement techniques in groundwater - pick the cross section location at } \\
\text { the downgradient edge of the vadose zone source using a width defined by the } \\
\text { width of higher-concentration groundwater data. }\end{array}$ & 3.1 \\
\hline SOURCE TYPE & DATA NEEDS & GO TO SECTION: \\
\hline Mass Flux & $\begin{array}{l}\text { Soil and groundwater concentration data, physical configuration of site (e.g., } \\
\text { thickness of vadose zone), historical site data. }\end{array}$ & 3.2 \\
\hline RECEPTOR/TRAVEL TIME & DATA NEEDS & GO TO SECTION: \\
\hline Qualitative Estimate & $\begin{array}{l}\text { Soil and groundwater concentration data, physical configuration of site (e.g., } \\
\text { thickness of vadose zone), historical site data. }\end{array}$ & 3.3 \\
\hline \multirow[t]{4}{*}{ Quantitative Estimate } & $\begin{array}{l}\text { Hydraulic conductivity from aquifer tests or literature values, hydraulic head for } \\
\text { at least two points parallel with the flow direction with a known distance } \\
\text { between the points, porosity estimate from particle size analysis, direct lab } \\
\text { tests, or from literature (such as the MNA Protocol, U.S. EPA, 1998). }\end{array}$ & \\
\hline & $\begin{array}{l}\text { Note: if the plume direction is not obvious, groundwater potentiometric maps } \\
\text { may be needed to interpret groundwater flow direction and to assess the } \\
\text { hydraulic gradient. In this case, numerical modeling may be necessary. }\end{array}$ & 3.3 \\
\hline & $\begin{array}{l}\text { Alternative method: a more refined approach can be applied if the } \\
\text { concentration vs. distance degradation rate of dissolved constituents that have } \\
\text { left a subsurface source is known. See U.S. EPA (2002) for information on } \\
\text { how to calculate a concentration vs. distance degradation rate. }\end{array}$ & \\
\hline & $\begin{array}{l}\text { Numerical modeling can provide an estimate of travel time if sufficient } \\
\text { information to describe the site, plume, and attenuation processes is available. }\end{array}$ & \\
\hline PLUME STABILITY & DATA NEEDS & GO TO SECTION: \\
\hline $\begin{array}{l}\text { Conventional } \\
\text { Assessment }\end{array}$ & $\begin{array}{l}\text { Concentration vs. time data to define changes in the plume extent and } \\
\text { contaminant distribution within the plume. }\end{array}$ & 3.4 \\
\hline $\begin{array}{l}\text { Additional quantitative } \\
\text { assessment for more } \\
\text { detailed MNA evaluation }\end{array}$ & $\begin{array}{l}\text { Concentration vs. time data for Mann-Kendall methods, MAROS software. } \\
\text { Contaminant distribution / hydrogeologic data for numerical/analytical } \\
\text { modeling. }\end{array}$ & 3.4 \\
\hline
\end{tabular}




\subsection{FINDING THE SCENARO}

To document the scenario selection process, use the worksheet on the next page. Record the hydrogeologic setting, geochemical setting, and modifying factors that best match your plume segment. Use Table 5 to determine the page number in the document for the scenario you selected.

TABLE 5. Scenario Lookup Table (To be Applied to Each Plume Segment)

\begin{tabular}{|c|c|c|c|}
\hline & G1. Anaerobic & G2. Anoxic & G3. Aerobic \\
\hline H1. Simple, faster flow regime & $\begin{array}{l}\text { Scenario 1. Go to page } \\
\qquad 1-1\end{array}$ & $\begin{array}{l}\text { Scenario 2. Go to page } \\
\text { S2-1 }\end{array}$ & $\begin{array}{c}\text { Scenario 3. Go to page } \\
\text { S3-1 }\end{array}$ \\
\hline H2. Simple, slower flow regime & $\begin{array}{l}\text { Scenario 4. Go to page } \\
\text { S4-1 }\end{array}$ & $\begin{array}{l}\text { Scenario 5. Go to page } \\
\text { S5-1 }\end{array}$ & $\begin{array}{l}\text { Scenario 6. Go to page } \\
\text { S6-1 }\end{array}$ \\
\hline $\begin{array}{l}\text { H3. Faster flow with significant } \\
\text { heterogeneities }\end{array}$ & $\begin{array}{c}\text { Scenario 7. Go to page } \\
\text { S7-1 }\end{array}$ & $\begin{array}{c}\text { Scenario 8. Go to page } \\
\text { S8-1 }\end{array}$ & $\begin{array}{c}\text { Scenario 9. Go to page } \\
\text { S9-1 }\end{array}$ \\
\hline $\begin{array}{l}\text { H4. Slower flow with significant } \\
\text { heterogeneities }\end{array}$ & $\begin{array}{l}\text { Scenario } 10 . \text { Go to page } \\
\text { S10-1 }\end{array}$ & $\begin{array}{l}\text { Scenario } 11 . \text { Go to page } \\
\text { S11-1 }\end{array}$ & $\begin{array}{c}\text { Scenario } 12 . \text { Go to page } \\
\text { S12-1 }\end{array}$ \\
\hline H5. Fractured or porous rock & & $\begin{array}{c}\text { Scenario } 13 . \text { Go to page } \\
\text { S13-1 }\end{array}$ & \\
\hline
\end{tabular}




\section{SCENARIO SELECTON WORKSHEET}

Divide Site into Plume Systems and then Plume Segments (Section 1.1 and Figure 1)

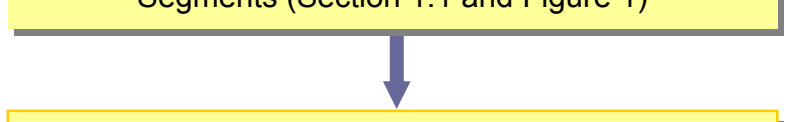

For Each Plume Segment, Select Hydrogeologic Setting and Geochemical Setting (Section 2.1 and 2.2)

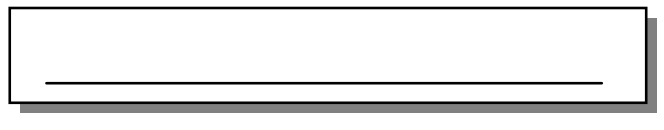

Plume Segment Name

H1. Simple, faster flow regime
H2. Simple, slower flow regime
H3. Faster flow with significant heterogeneities
H4. Slower flow with significant heterogeneities

Hydrogeologic Setting (select one)

G1. Anaerobic

G2. Anoxic

G3. Aerobic

Geochemical Setting (select one)

For Each Plume Segment, Determine Modifying Factors (if any) (Section 3)

For Each Plume Segment, Use Lookup Table to Find Page Number for Scenario (Section 4.0 and Table 5)

For Each Plume Segment, Go to Scenario Page and Get Scenario Specific Information Listed Above
Strong Source $\square$ Vadose Zone Source

Medium Source $\square$ Submerged Source

Weak Source $\square$ Mixed Source

Receptor is $<2$ years groundwater travel time.

Receptor is between 2 and 5 years travel time

Receptor is $>5$ years groundwater travel time

Plume Expanding or Perturbed

$\square$ Plume Stable or No Trend

$\square$ Plume Shrinking

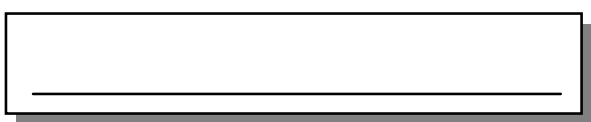

Scenario Name

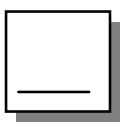

Page

Number

Figure 7. Scenario Selection Worksheet 


\subsection{WHAT YOU CAN LEARN FROM EACH SCENARIO}

Once you determine what scenario best matches your plume segment, you turn to the page where the scenario is located (see Table 5 for the page numbers). Each scenario is approximately ten pages long, and contains information that can be used to guide evaluation and implementation of MNA for a site that matches the basic characteristics of the scenario. The subsections of this chapter provide detailed background information for the scenarios topics, as identified in the outline below. Key information in each scenario includes the following:

- $\quad$ SCENARIO DESCRIPTION (for background information see Section 2)

- Hydrogeologic Setting

- Geochemical Setting

- KEY DECHLORINATION REACTIONS (for background information see Section 5.1)

- $\quad$ EFFECT OF MODIFYING FACTORS (for background information see Section 3)

- WILL MNA WORK?

- Potential for MNA Processes to Control Plume (for background information see Section 5.2)

- Key Sustainability Concept (for background information see Section 5.3)

- HOW DO I CHARACTERIZE THIS TYPE OF SITE?

- Actions Needed to Determine MNA Viability (for background information see Section 5.4)

- Key Monitoring Concepts (for background information see Section 5.5)

- Key Uncertainty Concepts (for background information see Section 5.6)

- HOW DO I ANALYZE DATA? (for background information see Section 5.7)

- WHAT ABOUT COSTS AND ENHANCEMENTS?

- Cost Considerations (for background information see Section 5.8)

- Key Enhanced Attenuation Concepts (for background information see Section 5.9)

- Key Source Control Concepts (for background information see Section 5.10)

\subsection{Dechlorination Reactions}

Selection of a scenario is dependent on some primary geochemical indicators because the geochemistry significantly impacts the type of degradation mechanisms that may be active at the site. The geochemical indicators usually define the dominant electron acceptors for bacteria and, therefore, categorize the overall activity of bacteria at the site. In general, the sequence of electron acceptors (non-contaminant) from more oxidizing, to more reducing conditions are: oxygen, nitrate, iron/manganese, sulfate, and carbon dioxide (methane production). Dechlorination reaction pathways and rates have been shown to vary as a function of the electron acceptor conditions at the site (see section 5.2). While there are other factors that can impact dechlorination activity of bacteria, these electron acceptors provide a primary means of categorizing what reactions can occur.

At one end of the geochemical spectrum is the aerobic geochemical setting where oxygen is present as the primary electron acceptor for subsurface bacteria. Because oxygen is generally preferred by bacteria over all other electron acceptors and is toxic to many anaerobic bacteria, the presence of oxygen defines a very specific set of bacterial activity. Once oxygen concentration drops below about $10 \%$ of the solubility limit, the activity of aerobic bacteria and the toxic effect of oxygen are greatly diminished. When oxygen is absent and there is sufficient substrate for anaerobic bacteria to flourish, there are clear end 
products that serve as indicators that significant anaerobic activity is occurring. Depending on the type of anaerobic bacteria that are dominating the subsurface, methane, reduced iron, and/or sulfide will be present and the concentrations of more oxidized electron acceptors such as nitrate and sulfate will be low. Under these anaerobic conditions, it has been demonstrated that dechlorination reactions usually occur in conjunction with the anaerobic activity that produces the indicator compounds. The anoxic geochemical condition describes the type of conditions where oxygen is not present at high enough levels to inhibit the activity of other bacteria, but there are no, or limited, indicators of significant activity of anaerobic bacteria. It is more difficult to determine the type of biological dechlorination reactions that are occurring at the site under anoxic conditions. However, biological dechlorination reactions may still be a significant attenuation mechanism. Typically under the anoxic geochemical setting, some additional information is needed to fully quantify the extent of biological dechlorination at the site. The scenarios cannot fully describe all of the information and analysis that is needed under these conditions, but do provide some guidance for how to proceed in obtaining this information.

Abiotic reactions can either be water-phase reactions, or are catalyzed by aquifer materials. Waterphase abiotic reactions are included as part of the reaction tables below. These reactions are usually not significantly impacted by the geochemical conditions, though the rate of reaction is a function of temperature and, in some cases, $\mathrm{pH}$. Catalyzed abiotic reactions are not included as a separate category below, but are considered as part of the reactions that are dependent on sediment components such as iron. These reactions are coupled with the corresponding biological reactions that reduce/oxidize aquifer sediment components. As such, catalyzed reactions are impacted by geochemical conditions. Abiotic catalyzed dechlorination reacitons typically require reducing conditions associated with the anoxic or anaerobic geochemical setting. However, dechlorination products from catalyzed abiotic reactions such as with reduced iron are different than the products observed in biological dechlorination. For instance, TCE can be degraded by reduced iron through a beta elimination reaction where the measurable end products typically include acetylene, ethene, ethane, and chloride. The abiotic catalyzed reactions, such as with reduced iron, are not included in the reaction tables and figures below, but may need to be considered for some sites.

Based on the characteristics of the geochemical settings, some dechlorination reactions are very likely to occur, some are very unlikely to occur, and some may occur depending on specific circumstances. Using figures, simple "Consumer Reports" indicators, and the appropriate geochemical setting for their site, the scenario user can determine what reactions are most likely and will also know what reactions are possible depending on more detailed information.

Figures 8 through 19 illustrate the dechlorination reactions that may occur at a site depending on the geochemical conditions and contaminants present. The figures show the possible reactions for each contaminant that are reported in the literature. Rates, in the form of the half-life in years at a temperature of $25^{\circ} \mathrm{C}$ and $\mathrm{pH}$ of 7 , are presented for water-phase abiotic reactions that always occur and are not significantly dependent on site conditions. Rates for the other biologically catalyzed reactions cannot be defined generically. Nomenclature and a description of each reaction are listed in Table 6 . References for laboratory data describing each reaction (except those noted as "highly unlikely") are provided corresponding to the footnote numbers shown in the figures. The references are not intended to represent an exhaustive literature review, but provide examples of laboratory information that is available to describe the reactions. For the geochemical setting categorization (see section 2.2), the anaerobic and aerobic settings are defined such that they represent conditions where it is highly likely that specific reactions are occurring. For some reactions, additional information is also needed under aerobic or anaerobic geochemical settings to determine whether the reaction is occurring at a site. The anoxic geochemical setting represents sites where the criteria used to define the general geochemical conditions are not sufficient to determine the specific reactions that are likely to occur. Thus, for anoxic geochemical settings, more detailed information is always needed to determine what reactions are occurring. Based on the nomenclature and description in Table 6, Table 7 describes the type of additional characterization information that is necessary to determine whether a reaction is occurring. 
TABLE 6. Description of Reactions

Reaction

Aerobic Co-Metabolism

Abbreviation

\section{Description}

Dechlorination of a compound where the compound is

ACM fortuitously degraded by an enzyme used in cellular metabolism - typically a monooxygenase enzyme.

Anaerobic Co-Metabolism

Dechlorination of a compound where the compound is fortuitously used as a surrogate electron acceptor, though the cell does not gain energy by reduction of the compound. For the reactions listed as ANCM, denitrification is an example metabolic process that supports this activity.

\begin{tabular}{lll}
\hline Aerobic Direct Metabolism & ADM & $\begin{array}{l}\text { Use of the chlorinated compound as an electron donor for } \\
\text { aerobic metabolism. }\end{array}$ \\
\hline Anaerobic Direct Metabolism & ANDM & $\begin{array}{l}\text { Use of the chlorinated compound as an electron donor for } \\
\text { anaerobic respiration - typically coupled to iron reduction. }\end{array}$ \\
\hline
\end{tabular}

Abiotic Hydrolysis

$\mathrm{AH}$

Homogeneous abiotic dechlorination - no specific reaction for this classification.

Dichloroelimination (biotic)

DC

Dechlorination of a compound where the compound is used as an electron acceptor, the bacteria may or may not gain energy by reduction of the compound. This reaction removes two chloride atoms in an elimination reaction. The more general term for this reaction is dihaloelimination.

Dehydrochlorination (abiotic)

$\mathrm{DHC}$

Reductive Dechlorination (hydrogenolysis) elimination reaction. This reaction is usually referred to as abiotic, but studies indicate that the reaction can be enhanced/catalyzed by bacteria and/or minerals (e.g., clay). The more general term for this reaction is dehydrohalogenation and is sometimes referred to as dehydrodehalogenation.

Dechlorination of a compound where the compound is used as an electron acceptor, the bacteria may or may not gain energy by reduction of the compound. This reaction removes one chloride atom from the compound and replaces it with a proton.
This reaction removes one chloride atom and one proton in an 
TABLE 7. Additional Characterization Information to Assess Whether a Reaction Will Occur

Reaction

Aerobic Co-Metabolism

Anaerobic Co-Metabolism

ANCM

\begin{abstract}
Aerobic Direct Metabolism
\end{abstract}
ADM

Anaerobic Direct Metabolism

ANDM

Abiotic Hydrolysis

Abiotic Hydrolysis

Dichloroelimination (biotic)

DC

Dehydrochlorination (abiotic)

DHC

\section{Abbreviation Characterization Information}

A source of methane or other co-substrates for these reactions that is migrating into an aerated portion of the aquifer needs to be present to provide the driving force for these reactions.

This type of reaction typically occurs with denitrification. Thus, evidence of active denitrification and an energy source to drive this reaction (e.g., organic acids) is needed to verify that this reaction is occurring.

No additional information is needed.

Anaerobic direct metabolism is typically linked to utilization of an electron acceptor such as iron. Thus, evidence of this type of reduction is needed to assess whether this reaction is occurring.

Confirm temperature and $\mathrm{pH}$ for use of half-life values in figures and to adjust as needed based on root data and equations in noted references.

This reaction occurs under geochemically reduced conditions. Thus, the site must meet most or all of the anaerobic setting criteria in Table 3 . The specific daughter products produced by $\mathrm{DC}$ should also be present in most cases. Especially under the anoxic geochemical setting, microcosm tests with site-specific sediments may be needed to verify this reaction.

Confirm temperature and $\mathrm{pH}$ for use of half-life values in figures and to adjust as needed based on root data and equations in noted references. This reaction may also be enhanced under geochemically reduced conditions.

Microcosm tests with site-specific sediments may be needed to verify any enhancement.

Reductive Dechlorination (hydrogenolysis)

This reaction occurs under geochemically reduced conditions. Thus, the site must meet most or all of the anaerobic setting criteria in Table 3. The specific daughter products produced by $\mathrm{RD}$ should also be present in most cases. Especially under the anoxic geochemical setting, microcosm tests with site-specific sediments may be needed to verify this reaction. 


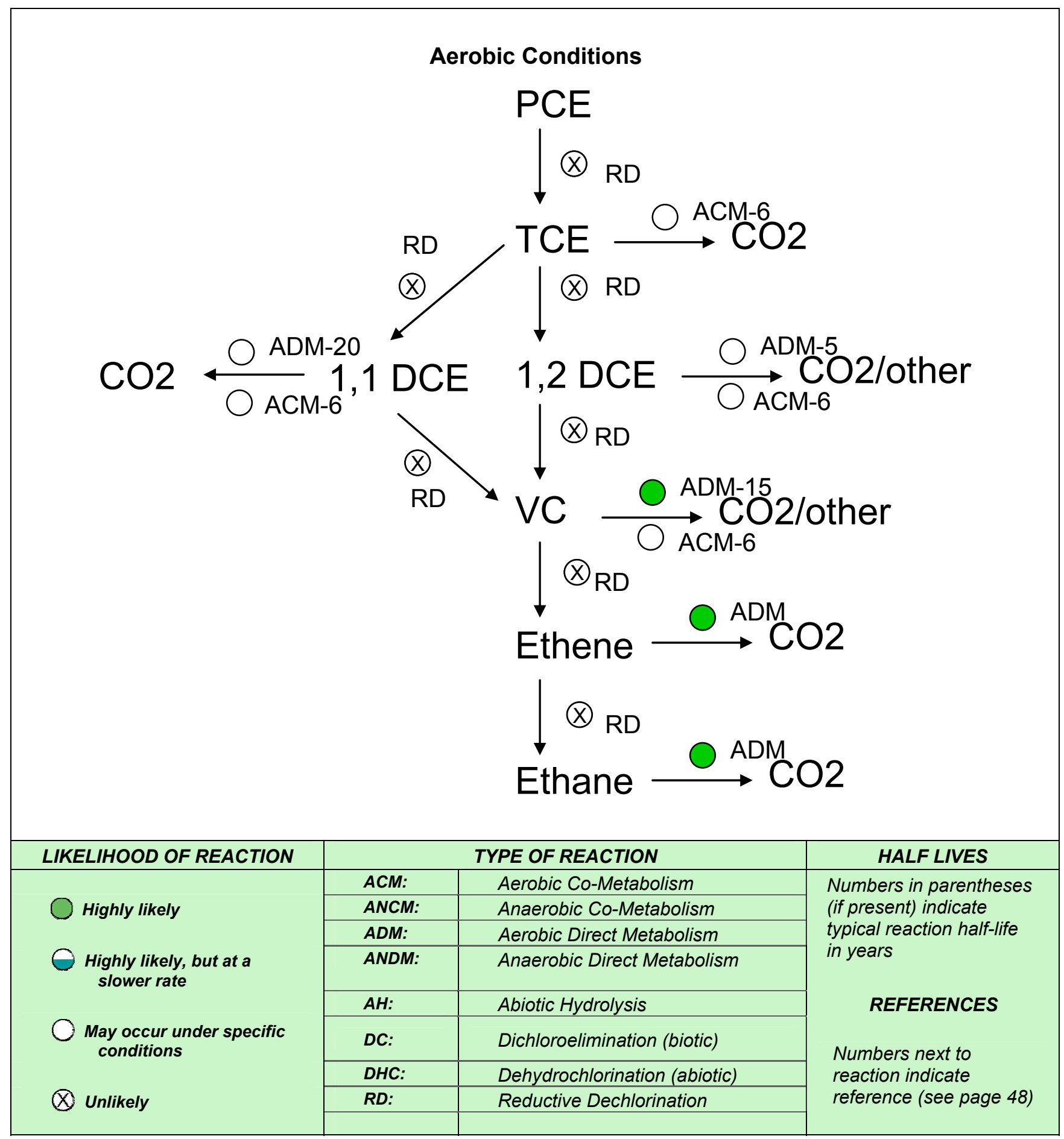

FIGURE 8. Dechlorination Reactions for PCE Under the Aerobic Geochemical Setting. 


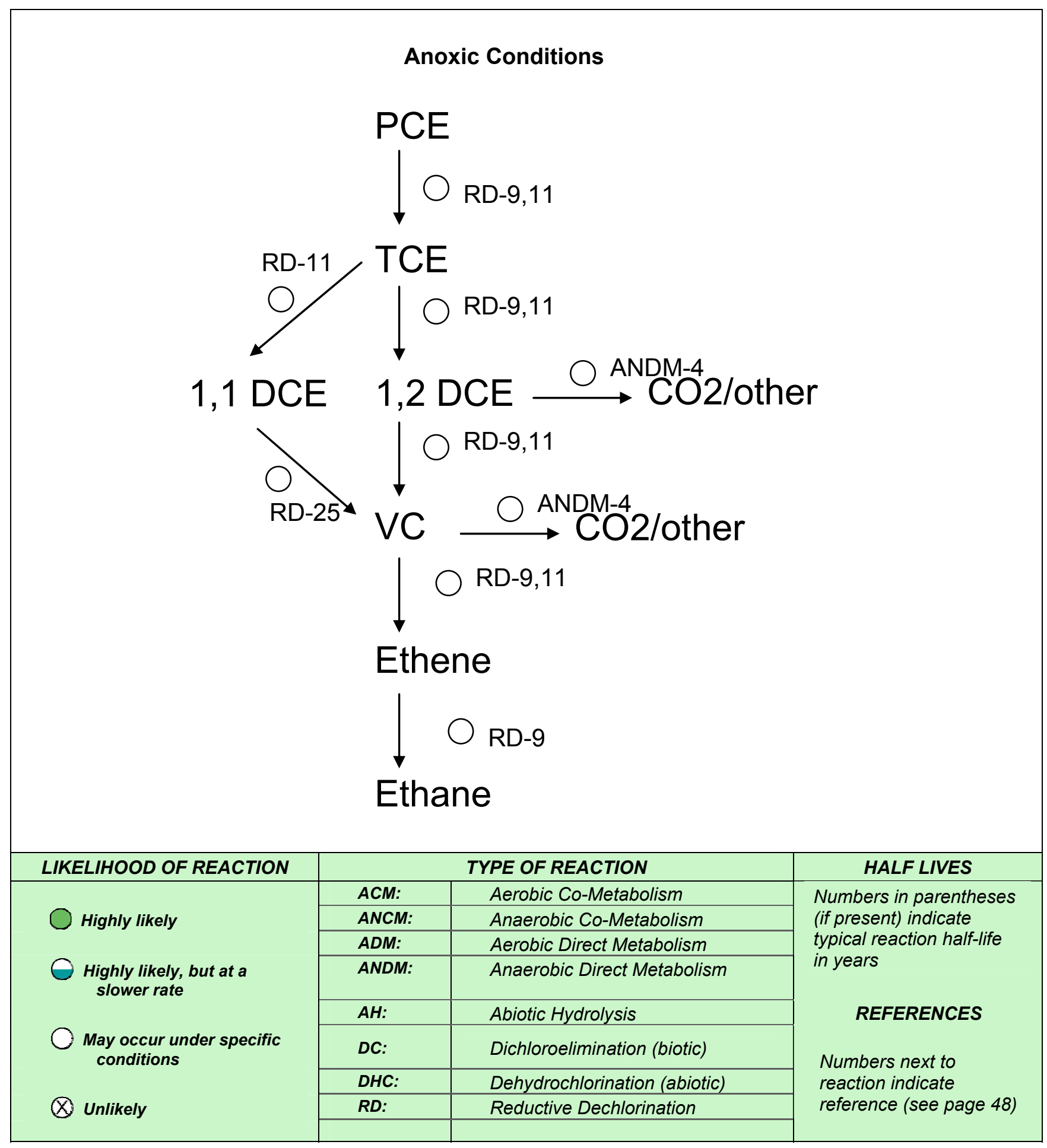

FIGURE 9. Dechlorination Reactions for PCE Under the Anoxic Geochemical Setting. 


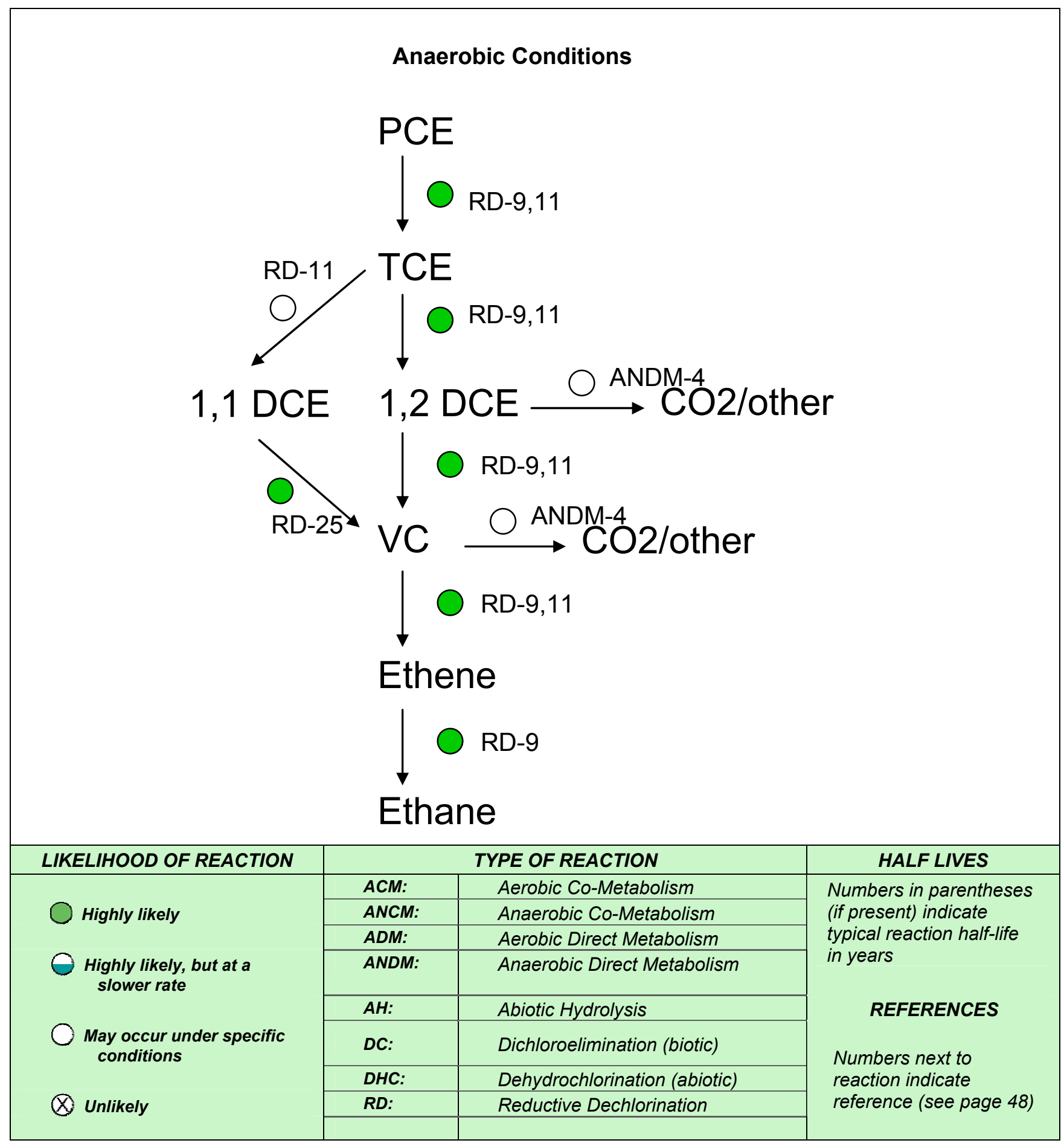

FIGURE 10. Dechlorination Reactions for PCE Under the Anaerobic Geochemical Setting. 


\section{Aerobic Conditions}

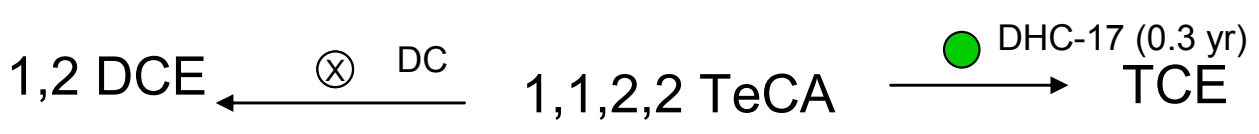

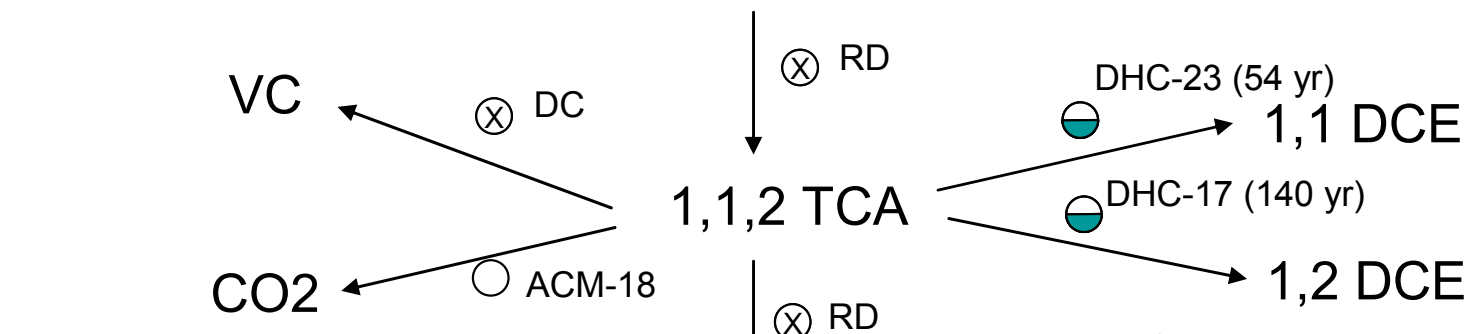

$\otimes R D$
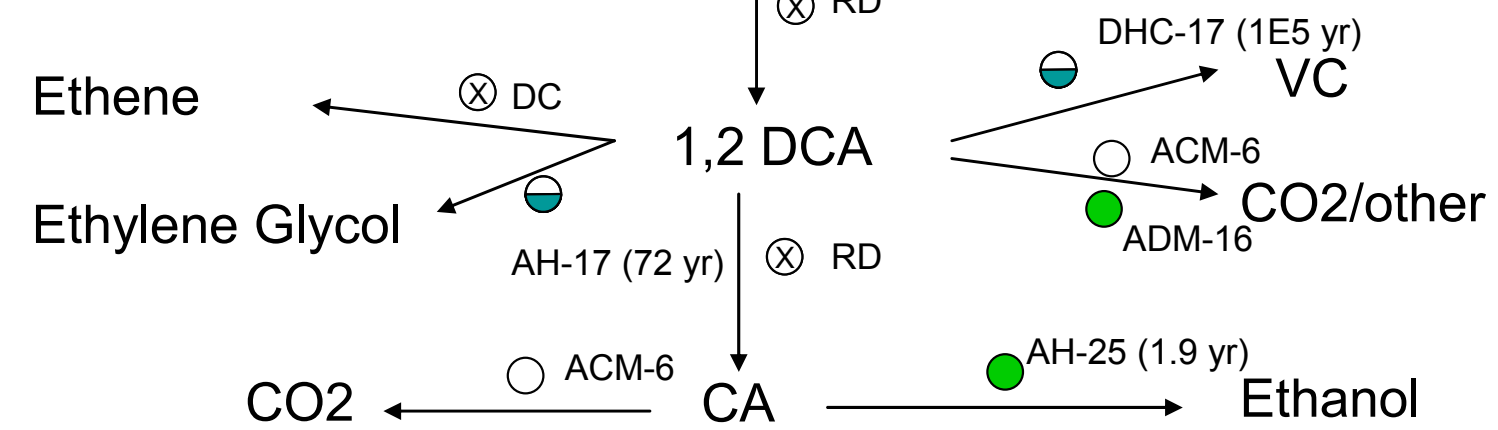

$\otimes$ RD

\section{Ethane}

\begin{tabular}{|c|c|c|c|}
\hline LIKELIHOOD OF REACTION & & YPE OF REACTION & HALF LIVES \\
\hline \multirow{3}{*}{ Highly likely } & ACM: & Aerobic Co-Metabolism & \multirow{4}{*}{$\begin{array}{l}\text { Numbers in parentheses } \\
\text { (if present) indicate } \\
\text { typical reaction half-life } \\
\text { in years }\end{array}$} \\
\hline & ANCM: & Anaerobic Co-Metabolism & \\
\hline & ADM: & Aerobic Direct Metabolism & \\
\hline \multirow{2}{*}{$\begin{array}{c}\text { Highly likely, but at a } \\
\text { slower rate }\end{array}$} & ANDM: & Anaerobic Direct Metabolism & \\
\hline & AH: & Abiotic Hydrolysis & \multirow{4}{*}{$\begin{array}{l}\text { REFERENCES } \\
\text { Numbers next to } \\
\text { reaction indicate } \\
\text { reference (see page 48) }\end{array}$} \\
\hline \multirow{3}{*}{$\begin{array}{l}\text { May occur under specific } \\
\text { conditions }\end{array}$} & $D C:$ & Dichloroelimination (biotic) & \\
\hline & DHC: & Dehydrochlorination (abiotic) & \\
\hline & RD: & Reductive Dechlorination & \\
\hline
\end{tabular}

FIGURE 11. Dechlorination Reactions for 1,1,2,2-TeCA Under the Aerobic Geochemical Setting. 


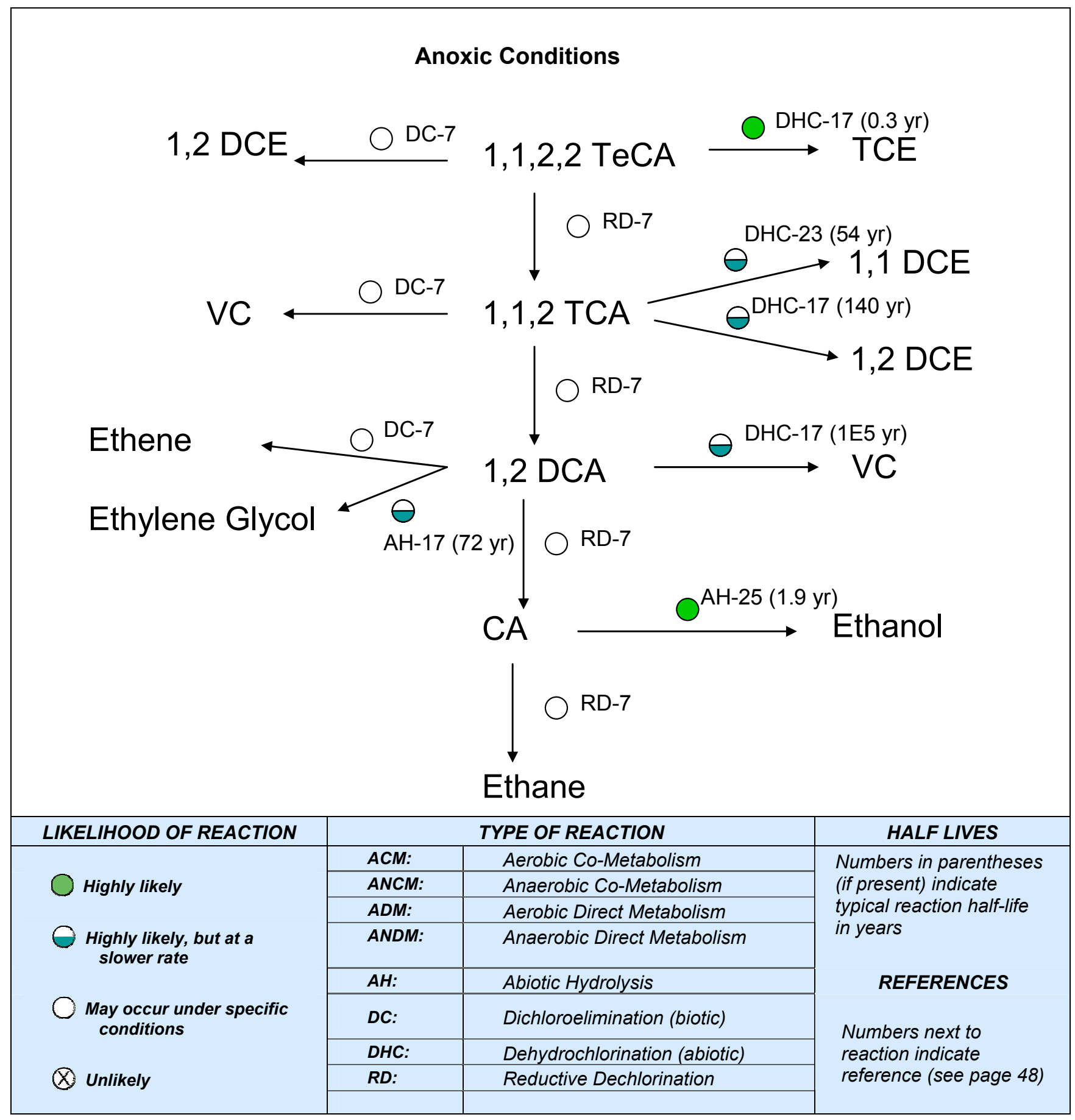

FIGURE12. Dechlorination Reactions for 1,1,2,2-TeCA Under the Anoxic Geochemical Setting. 


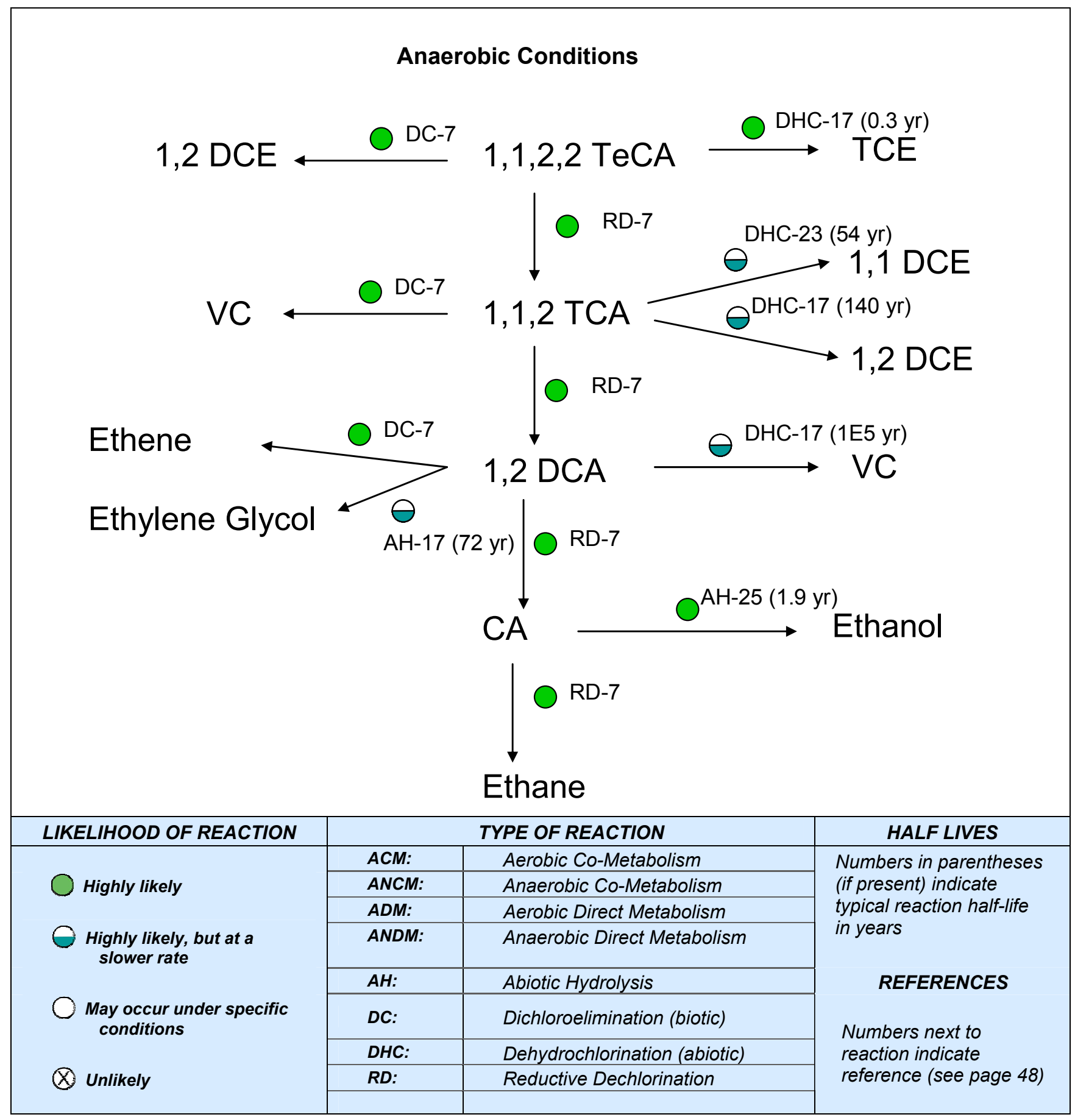

FIGURE 13. Dechlorination Reactions for 1,1,2,2-TeCA Under the Anaerobic Geochemical Setting. 


\section{Aerobic Conditions}
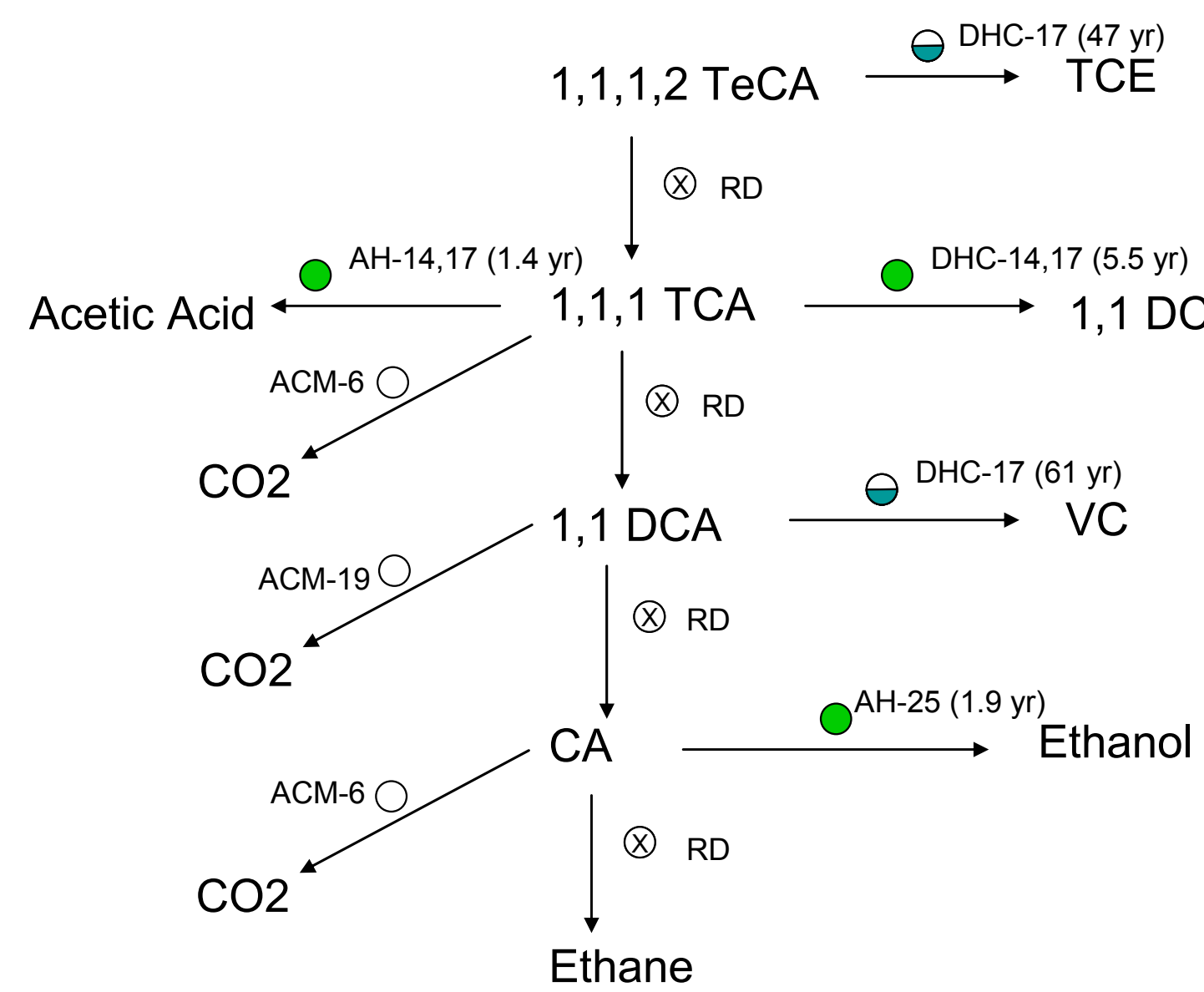

\begin{tabular}{|c|c|c|}
\hline LIKELIHOOD OF REACTION & & PPE OF REACTION \\
\hline \multirow{3}{*}{ Highly likely } & ACM: & Aerobic Co-Metabolism \\
\hline & ANCM: & Anaerobic Co-Metabolism \\
\hline & ADM: & Aerobic Direct Metabolism \\
\hline \multirow{2}{*}{$\begin{array}{l}\text { Highly likely, but at a } \\
\text { slower rate }\end{array}$} & ANDM: & Anaerobic Direct Metabolism \\
\hline & $A H:$ & Abiotic Hydrolysis \\
\hline \multirow{2}{*}{$\begin{array}{l}\text { May occur under specific } \\
\text { conditions }\end{array}$} & $D C:$ & Dichloroelimination (biotic) \\
\hline & DHC: & Dehydrochlorination (abiotic) \\
\hline$\bigotimes$ Unlikely & $R D:$ & Reductive Dechlorination \\
\hline
\end{tabular}

HALF LIVES

Numbers in parentheses (if present) indicate typical reaction half-life in years

\section{REFERENCES}

Numbers next to reaction indicate reference (see page 48)

FIGURE 14. Dechlorination Reactions for 1,1,1,2-TeCA Under the Aerobic Geochemical Setting. 


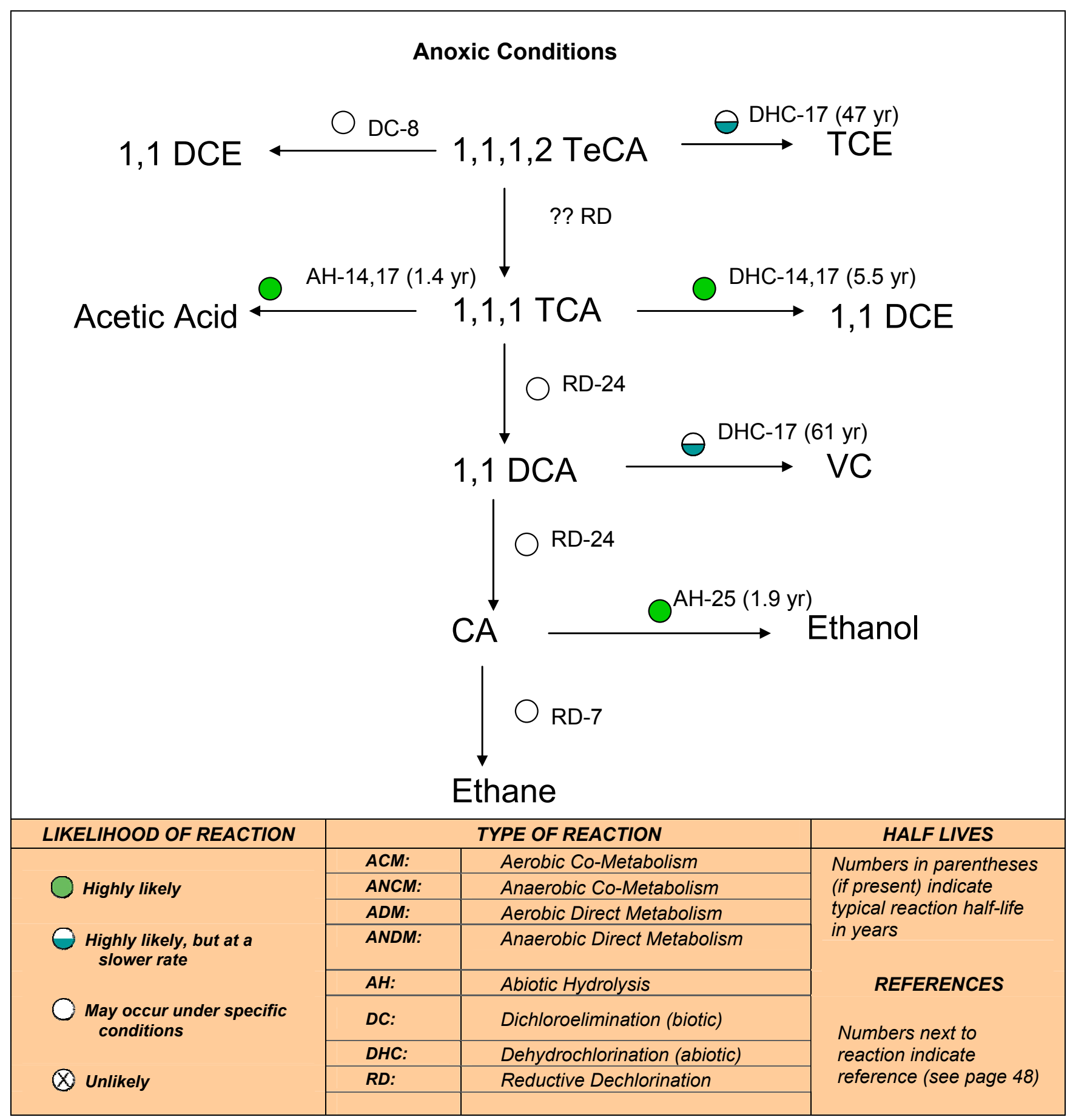

FIGURE 15. Dechlorination Reactions for 1,1,1,2-TeCA Under the Anoxic Geochemical Setting. 


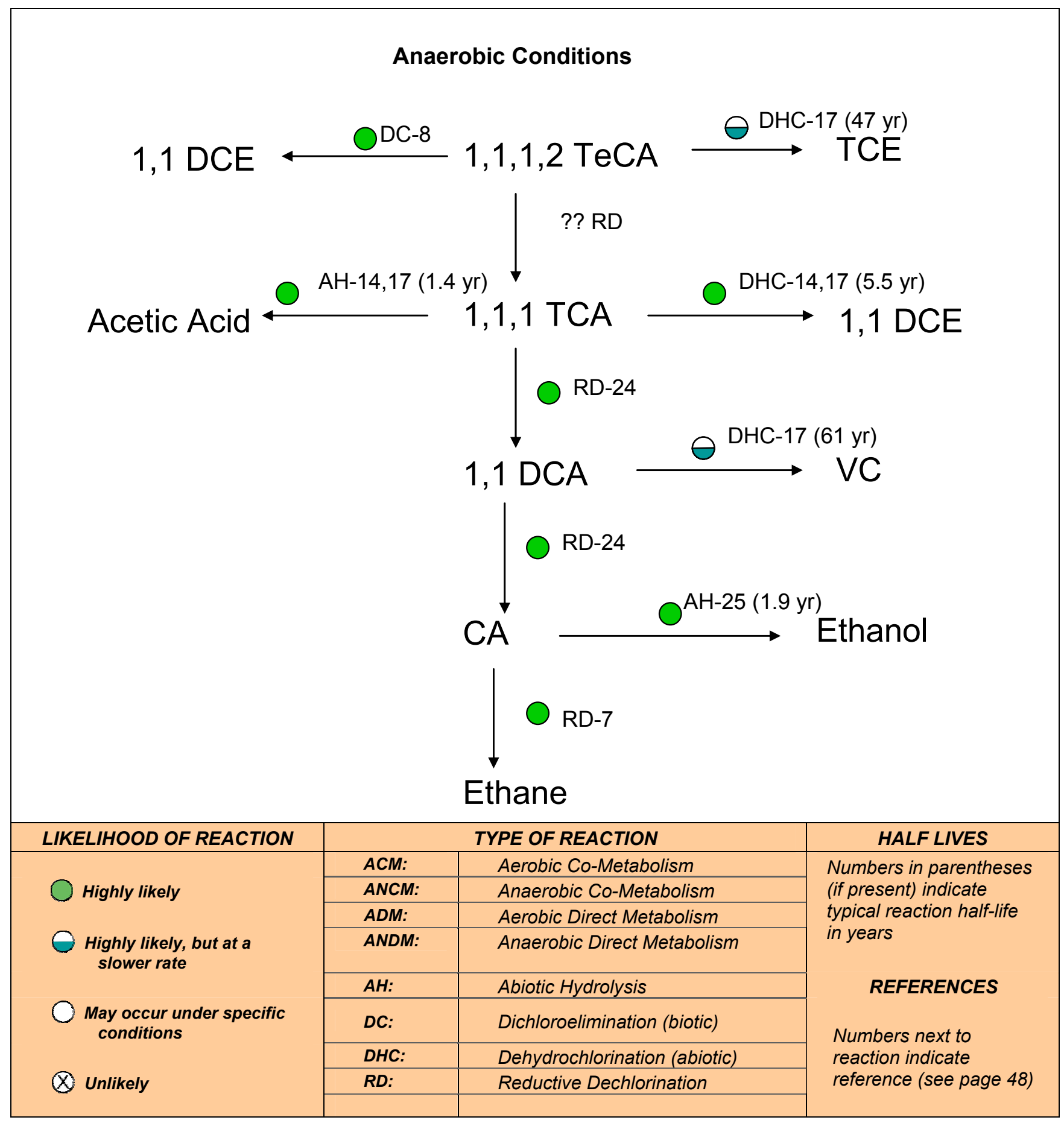

FIGURE 16. Dechlorination Reactions for 1,1,1,2-TeCA Under the Anaerobic Geochemical Setting. 


\section{Aerobic Conditions}

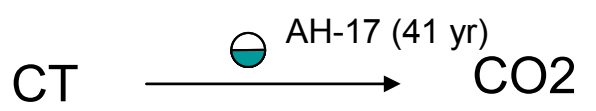

$\otimes \mathrm{RD}$

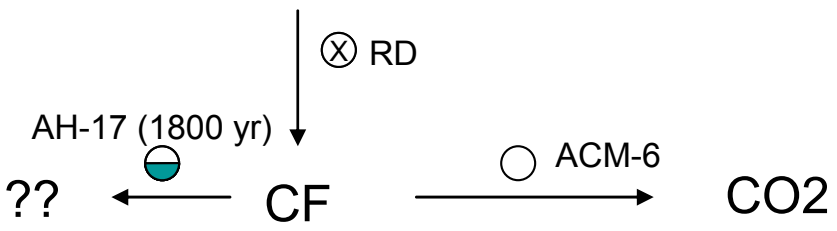

$\otimes$ RD
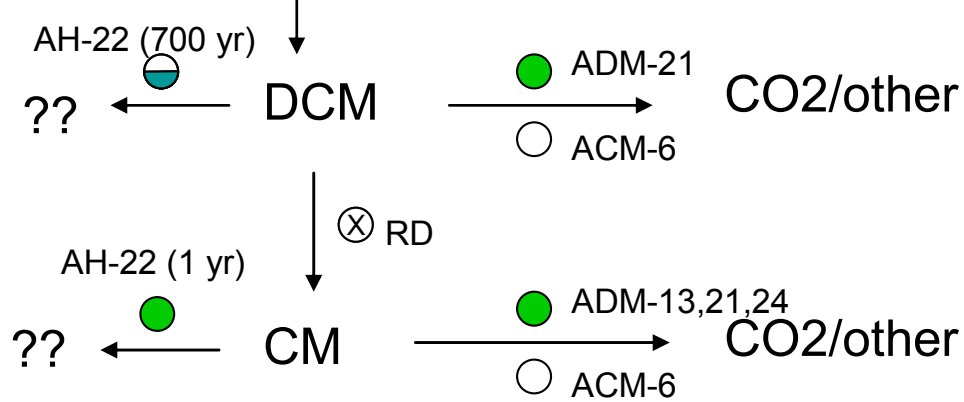

$\otimes \mathrm{RD}$

\section{$\mathrm{CH} 4$}

\begin{tabular}{|c|c|c|c|}
\hline LIKELIHOOD OF REACTION & & IPE OF REACTION & HALF LIVES \\
\hline \multirow{3}{*}{ Highly likely } & ACM: & Aerobic Co-Metabolism & \multirow{4}{*}{$\begin{array}{l}\text { Numbers in parentheses } \\
\text { (if present) indicate } \\
\text { typical reaction half-life } \\
\text { in years }\end{array}$} \\
\hline & ANCM: & Anaerobic Co-Metabolism & \\
\hline & ADM: & Aerobic Direct Metabolism & \\
\hline \multirow{2}{*}{$\begin{array}{l}\text { Highly likely, but at a } \\
\text { slower rate }\end{array}$} & ANDM: & Anaerobic Direct Metabolism & \\
\hline & AH: & Abiotic Hydrolysis & \multirow{4}{*}{ reference (see page 48) } \\
\hline $\begin{array}{l}\text { May occur under specific } \\
\text { conditions }\end{array}$ & $D C:$ & Dichloroelimination (biotic) & \\
\hline \multirow[b]{2}{*}{ ( U) Unlikely } & DHC: & Dehydrochlorination (abiotic) & \\
\hline & RD: & Reductive Dechlorination & \\
\hline
\end{tabular}

FIGURE 17. Dechlorination Reactions for CT Under the Aerobic Geochemical Setting. 


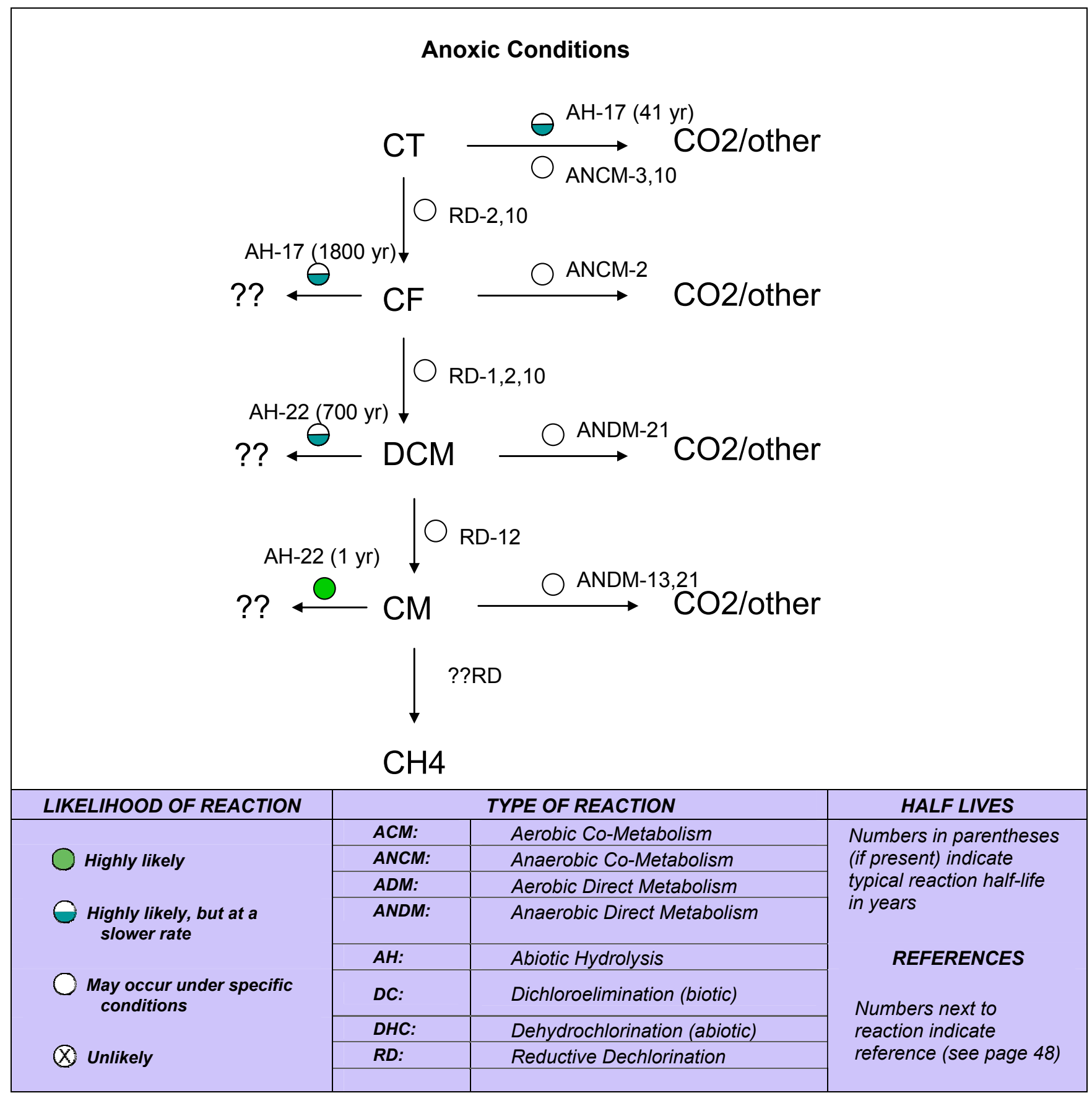

FIGURE 18. Dechlorination Reactions for CT Under the Anoxic Geochemical Setting. 


\section{Anaerobic Conditions}
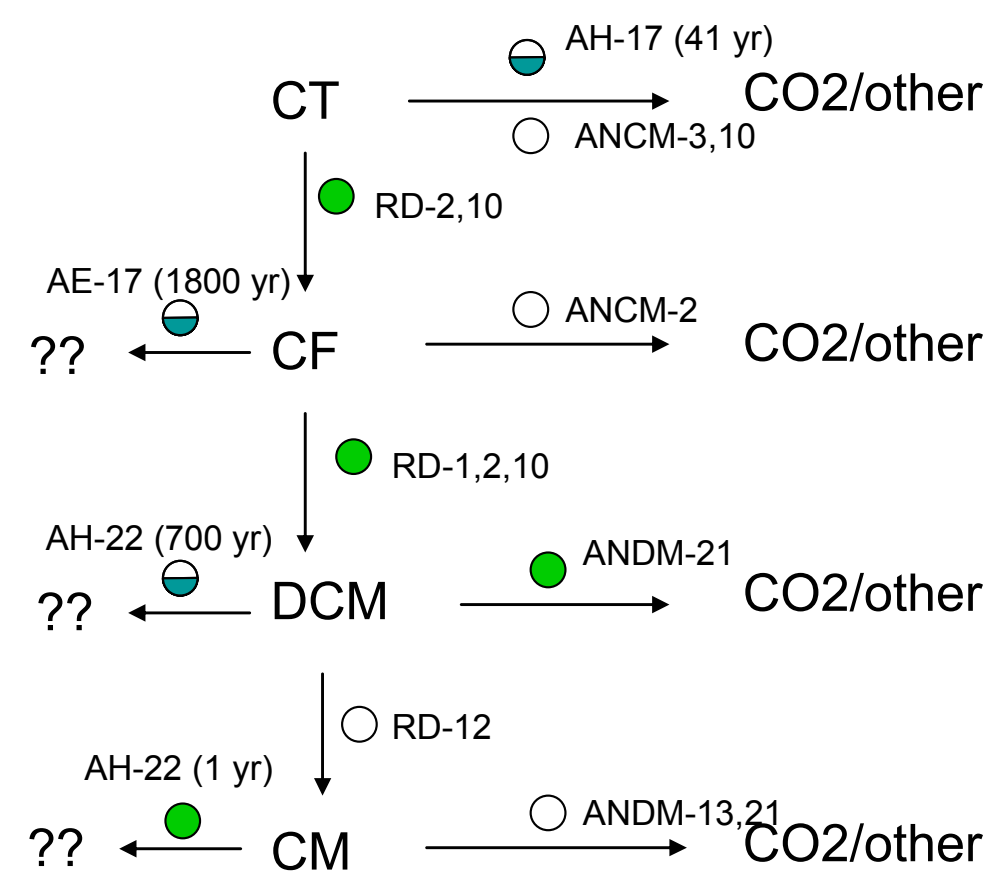

??RD

\section{$\mathrm{CH} 4$}

\begin{tabular}{|c|c|c|c|}
\hline LIKELIHOOD OF REACTION & & YPE OF REACTION & HALF LIVES \\
\hline \multirow{3}{*}{ Highly likely } & ACM: & Aerobic Co-Metabolism & \multirow{4}{*}{$\begin{array}{l}\text { Numbers in parentheses } \\
\text { (if present) indicate } \\
\text { typical reaction half-life } \\
\text { in years }\end{array}$} \\
\hline & ANCM: & Anaerobic Co-Metabolism & \\
\hline & ADM: & Aerobic Direct Metabolism & \\
\hline \multirow{2}{*}{$\begin{array}{l}\text { Highly likely, but at a } \\
\text { slower rate }\end{array}$} & ANDM: & Anaerobic Direct Metabolism & \\
\hline & AH: & Abiotic Hydrolysis & \multirow[b]{4}{*}{ reference (see page 48) } \\
\hline \multirow{2}{*}{$\begin{array}{l}\text { May occur under specific } \\
\text { conditions }\end{array}$} & $D C:$ & Dichloroelimination (biotic) & \\
\hline & DHC: & Dehydrochlorination (abiotic) & \\
\hline$\bigotimes$ Unlikely & RD: & Reductive Dechlorination & \\
\hline
\end{tabular}

FIGURE 19. Dechlorination Reactions for CT Under the Anaerobic Geochemical Setting. 


\subsection{Key Processes: Potential for MNA Processes to Control the Plume}

This part of the scenario summarizes the relative importance of advection, dispersion, sorption, and degradation as natural attenuation processes and describes how mass balance concepts can be used in the scenario. For mass balance to be useful in engineering practice, however, it is necessary to quantify it in practical ways that facilitate overall site remediation and which are consistent with existing regulatory guidance.

One companion tool to this scenarios based approach is the BIOBALANCE software system ${ }^{1}$ This tool describes

- which processes contribute how much to the overall assimilative capacity (i.e., linear sorption, biodegradation, abiotic degradation, dispersion, dilution);

- how the source term might change over the long-term lifetime of the source;

- to what degree competing reactions interfere with solvent biodegradation processes; and

- how sustainable biodegradation via reductive dechlorination is likely to be over the long term.

${ }^{1}$ Developed by Groundwater Services (www.gsi-net.com), with the support of the Savannah River National Laboratory and DOE,to evaluate monitored natural attenuation at chlorinated solvent sites.

\subsection{Key Sustainability Concept}

This part of the scenario summarizes what can be determined about the sustainability of the key processes over the long term. The United States National Research Council (NRC) describes sustainability as occurring when the rates of the protective mechanisms continue to equal the rate at which the contaminants enter the groundwater (NRC, 2000). While the U.S. Environmental Protection Agency's Monitored Natural Attenuation Directive does not refer to sustainability directly, it does conclude that the effectiveness/applicability of MNA in the near and long term should be demonstrated to EPA (or other overseeing regulatory authority) through: 1) sound technical analyses which provide confidence in natural attenuation's ability to achieve remediation objectives; 2) performance monitoring; and 3) contingency (or backup) remedies where appropriate(U.S. EPA, 1999). The BIOBALANCE software system has a software module designed to evaluate sustainability issues (www.gsi-net.com).

Different attenuation mechanisms have different potentials for being sustainable or not sustainable, as described in Table 8 and Figure 20.

TABLE 8. Sustainability of Attenuation Processes.

\begin{tabular}{ll} 
Attenuation mechanism & Sustainability Potential \\
Dispersion & $\begin{array}{l}\text { Cannot be stopped. Will continue as long as } \\
\text { dissolved plume is present. Can produce steady- } \\
\text { state plume. }\end{array}$ \\
\hline Sorption & $\begin{array}{l}\text { Sorption slows development of the plume and } \\
\text { sorbed materials will be released to groundwater } \\
\text { when groundwater concentrations decrease. }\end{array}$ \\
\hline Hydrolysis & $\begin{array}{l}\text { Cannot be stopped. Will continue as long as } \\
\text { dissolved plume is present. }\end{array}$ \\
\hline Aerobic Degradation & $\begin{array}{l}\text { Based on presence of oxygen. Very unlikely to stop } \\
\text { unless new source upgradient removes oxygen, or } \\
\text { hydrologic changes occur to divert oxygen away } \\
\text { from source/plume. }\end{array}$ \\
\hline Anoxic Degradation & $\begin{array}{l}\text { For aerobic-related reactions, see row above. For } \\
\text { anaerobic-related reactions, see row below. }\end{array}$ \\
\hline Anaerobic Degradation & $\begin{array}{l}\text { See framework below for potential sustainability } \\
\text { types for anaerobic reactions. }\end{array}$ \\
\hline
\end{tabular}


The framework shown below is for anaerobic reactions at chlorinated solvent sites (Newell and Aziz, 2004). Note that specific reactions requires that the specific metabolic capacity exist in the plume segment as well as the presence of an electron donor.

- Sustainability Type A: The available donor ("AD") is always present in lower available equivalents than the solvent ("S"), resulting in a solvent plume that is uncontrolled by biodegradation over the short-term and long-term.

- Sustainability Type B: The available donor ("AD") is always present in higher available equivalents than the solvent ("S"), resulting in a solvent plume that is controlled by biodegradation over the short term and the long-term.

- Sustainability Type C ("Take-Over"): The available donor (“AD”) starts out with lower available equivalents than the solvent ("S"), but the rate of concentration decline for solvents is greater than the rate of decline for the donor. Therefore, at some point in the future biodegradation will "take over" control of the plume and the plume will eventually stabilize and shrink.

- Sustainability Type D (“Burn-Out”): The opposite of "Take-Over", where the decline in donor concentrations is greater than the decline in concentrations in solvents. Therefore, a plume that is controlled in the beginning of the plume's lifetime becomes uncontrolled at some point in the future, resulting in an expanding plume.

- Sustainability Type E: This curve describes a Type 3 solvent site, where no donor is available. The plume is uncontrolled by biodegradation (but will eventually be controlled by dispersion).

- Sustainability Type F: This curve describes the case where solvent and donor both exhibit the same decline curve, resulting in a long-term stable condition. This condition is unlikely at actual sites. 


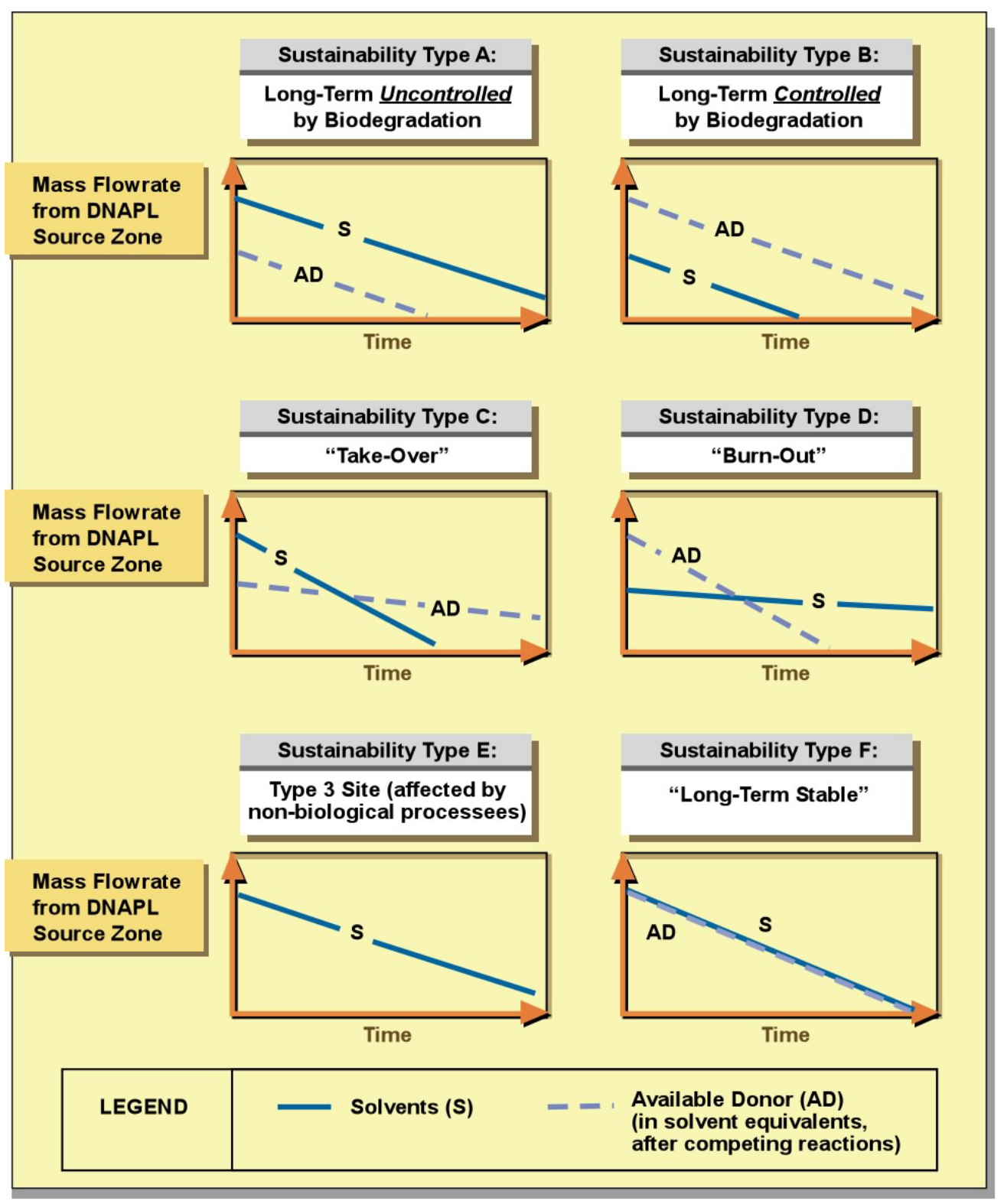

FIGURE 20. Framework for Sustainability of Anaerobic Reactions at Chlorinated Solvent Sites (from Newell and Aziz, 2004)

Sustainability Types B, C, and F are sustainable over the lifetime of a chlorinated solvent plume undergoing reductive dechlorination. Sustainability Types $A, D$, and $E$ are not sustainable. The linear decline curves on each graph in Figure 20 are conceptual only, and the actual decline curve will likely have a more complicated pattern than a constant decline. For example, a Type 2 site with excess donor could be described by a Type B curve, except that the line marked "AD" would be a straight line as the concentration of the electron donor (naturally occurring organics in upgradient groundwater) would remain relatively constant over time. 


\subsection{Actions Needed to Determine MNA Viability}

This part of the scenario summarizes what you can do to determine if MNA is a viable management strategy for a plume segment. The evaluation of MNA essentially requires that "lines of evidence" are established to assess whether natural attenuation can meet the remediation objectives for the site. The key lines of evidence are summarized below. Individual scenarios will provide information about the level of detail needed in the evaluation approach and what types of evidence may be most appropriate to support the evaluation.

\section{KEY POINT:}

This guide does not include information on how these measurements are actually made (i.e., test numbers, issues with sampling, etc.).

Refer to the existing protocols (such as the U.S. EPA MNA Protocol (1998) to get detailed information on test methods and sampling procedures.

\section{Mass Loss:}

The most preferred validation for natural attenuation is direct evidence that the plume is stable and shrinking without impacting any receptors. Especially for plumes in simple hydrogeologic and geochemical settings, mass loss can be assessed through the collection and analysis of groundwater monitoring data (see section 5.5). In more complex hydrogeologic and geochemical settings and for plumes for which a remedy currently exists (e.g., pump-and-treat), mass loss information may be difficult or impossible to obtain. When it is not possible to obtain convincing mass loss evidence, the next two lines of evidence can be used to evaluate MNA as a remedy.

\section{Geochemical Footprints:}

Daughter product production: For attenuation involving contaminant dechlorination, the type of daughter products present can indicate the specific reactions that are occurring. As shown in the dechlorination charts of section 5.1, different types of reactions can produce different daughter products. Presence of a specific dechlorination chain of parent and daughter products can be used as direct evidence that attenuation is occurring. However, it is important to know what species are present in the source material to interpret whether "daughter" products are present as a result of dechlorination reactions or due to contaminant migration from the source. Presence of daughter products indicates that an attenuation reaction is occurring, but additional analyses are necessary to determine the rate of attenuation. Presence of dechlorination end products such as ethene, ethane, or chloride, if at concentrations significantly higher than background, are good indicators that dechlorination has occurred.

Geochemical Setting: As discussed in section 2, geochemical indicators such as dissolved oxygen, nitrate, sulfate, methane, sulfide, ferrous iron, and ORP are useful in determining whether the conditions within the plume are suitable for specific types of dechlorination reactions. Thus, these geochemical setting data are useful in conjunction with other data to demonstrate that a specific dechlorination is occurring.

\section{Model/special study}

Microcosm Study: It is not always possible to determine the type of dechlorination attenuation process occurring at a site based on field data. While laboratory studies cannot exactly replicate field conditions, they can approximate field conditions and provide insight into the dechlorination attenuation mechanisms. Absolute rates of attenuation from laboratory studies are typically not expected to represent absolute rates under field conditions (except for some abiotic reactions). Relative rates, for instance for parent and daughter product dechlorination and the extent of dechlorination, can be reasonably approximated from laboratory data. Because of the controlled experimental conditions, detailed data analysis to determine the reaction pathways and rates is possible and provides useful information in terms of these relative dechlorination rates.

Genetic probes: In some cases it may be useful to understand the microbial community at a site to help interpret site chemical data and predict the type of dechlorination processes and related end products that are active and will continue to attenuate the plume. For instance, if a plume currently shows DCE, VC, and ethene as daughter products, it may be reasonable to ascertain the relative activity of organisms 
such as Dehalococcoides sp. that are able to fully dechlorinate chloroethenes to ethene/ethane. Microbial community analysis (e.g., T-RFLP, DGGE) can be used to determine the dominant microbes present and, by inference, the dominant geochemical conditions and biogeochemical/contaminant reactions can be estimated.

Modeling: The type of model applied at a specific site is dependent on the site conditions and the intended use of the model. The discussion here is limited to models for solute transport under saturated conditions. Two basic levels of models are available that are relevant to MNA modeling. Analytical models are capable of solving the general transport equation with specific limitations. Three-dimensional multi-species reactive transport numerical models discretize the transport equation and iteratively solve it within a defined numerical domain. Numerical models allow for more detailed configuration of the model domain to more closely match site features and, therefore, have advantages for some sites. Selection of the appropriate model for a specific site is dependent on the site conditions and configuration-related differences between analytical models and numerical models. Table 9 provides a brief overview of considerations for selecting the primary type of modeling analysis based on site properties, in particular based on whether the geochemistry and hydrology of the site readily supports a relatively simple description of attenuation and transport processes or the geochemistry and hydrology is complex. Other considerations for model selection are discussed below.

TABLE 9. Considerations for Selecting Modeling Approach Based on Site Properties

\begin{tabular}{|c|c|c|c|c|c|c|}
\hline \multirow[b]{2}{*}{$\begin{array}{l}\text { Modeling } \\
\text { Approach }\end{array}$} & \multicolumn{2}{|c|}{$\begin{array}{l}\text { Sites with supportive } \\
\text { geochemical / hydrologic conditions }\end{array}$} & \multicolumn{4}{|c|}{$\begin{array}{l}\text { Sites with hydrologic and / or } \\
\text { geochemical complexity / challenges }\end{array}$} \\
\hline & $\begin{array}{l}\text { Simple site with } \\
\text { stable or } \\
\text { shrinking plume }\end{array}$ & $\begin{array}{l}\text { Plume stability \& } \\
\text { geochemical } \\
\text { footprints uncertain }\end{array}$ & $\begin{array}{l}\text { Documented } \\
\text { growth or out } \\
\text { perturbed - } m \\
\text { stable in the } f\end{array}$ & $\begin{array}{l}\text { lume } \\
\text { rop or } \\
\text { ay be } \\
\text { iture }\end{array}$ & $\begin{array}{l}\text { Geochemical } \\
\text { conditions uncertain } \\
\text { and/or complex } \\
\text { hydrologic conditions }\end{array}$ & $\begin{array}{l}\text { Attenuation } \\
\text { Process } \\
\text { Enhancement } \\
\text { Evaluation }\end{array}$ \\
\hline $\begin{array}{l}\text { Conceptual Model - } \\
\text { Identify contributing } \\
\text { processes and the } \\
\text { active zones within } \\
\text { a plume. }\end{array}$ & \multicolumn{2}{|l|}{0} & \multicolumn{2}{|r|}{ [2] } & [2] & [2] \\
\hline $\begin{array}{l}\text { Conceptual Model - } \\
\text { plus } \\
\text { Analytical Model or } \\
\text { Mass Balance } \\
\text { Calculation }\end{array}$ & \multicolumn{2}{|l|}{0} & \multicolumn{2}{|l|}{0} & 0 & 0 \\
\hline $\begin{array}{l}\text { Conceptual Model, } \\
\text { possible Analytical } \\
\text { Model, and } \\
\text { Numerical Model }\end{array}$ & [1] & 0 & \multicolumn{2}{|l|}{0} & 0 & 0 \\
\hline $\mathrm{O}$ & \multicolumn{3}{|c|}{$\begin{array}{l}\text { NOTES: } \\
1 \text { Numerical modeling is not necessarily } \\
\text { preferred because costs may not be } \\
\text { justifiable for the offsetting benefits in terms } \\
\text { of uncertainty reduction, monitoring } \\
\text { optimization, etc. However, numerical } \\
\text { models may be selected if it is necessary to } \\
\text { provide better estimates of time frames and } \\
\text { better assurance of meeting certain types } \\
\text { of remediation goals (e.g., concentration }\end{array}$} & \multicolumn{3}{|c|}{$\begin{array}{l}\text { targets) than can be obtained with } \\
\text { analytical modeling. } \\
2 \text { Conceptual models are good to use for } \\
\text { planning and site management, but may } \\
\text { not be suited as primary support for decision } \\
\text { making at complex sites or sites that have high } \\
\text { uncertainty because conceptual models do } \\
\text { not allow testing of uncertainty and parameter } \\
\text { sensitivity and do not strongly support a } \\
\text { detailed evaluation of enhancements. }\end{array}$} \\
\hline
\end{tabular}


Analytical models such as BIOCHLOR (Aziz et al., 1999) have been established specifically for use in modeling MNA. For analytical models, the solution technique typically requires assumptions of uniform hydraulic properties throughout the domain, uniform steady-state groundwater flow (in some cases limited to one-dimensional advection), simple boundary conditions, simple source geometry, first-order contaminant transformation with rates constant within a defined area (in some cases for a single decay pathway), and uniform linear equilibrium partitioning. Analytical models can be useful in providing estimates of contaminant migration for plumes where these assumptions can be technically supported based on the site conditions. For instance, consider a plume with a well-defined contaminant source of TCE within a relatively homogeneous, thin aquifer that is bounded by aquitards or an aquitard and the water table where the aquifer has relatively constant methanogenic conditions throughout the plume. In this case, the assumptions required for use of an analytical model are appropriate.

Numerical models may be needed when site conditions cannot be described under the simplified flow, reaction, or adsorption process assumptions required for use of analytical models. The groundwater flow system at a site may not be uniform because of a complex distribution of hydraulic conductivity, complex recharge/discharge elements, or transient flow conditions. Sources distributed in multiple locations, multiple contaminant species with multiple reaction pathways, and multiple oxidation/reduction conditions within the plume area cause complexities in modeling the reaction processes at a site. In some cases, assumption of linear equilibrium sorption is not appropriate depending on the nature of the contaminant and the aquifer solids. For site conditions that include any or all of these complexities, numerical models are more appropriate than analytical models. For instance, the publicly-available Reactive Transport in 3Dimensions (RT3D) code (Clement et al., 1998; Clement, 1997) provides a framework to solve for reactive transport under these more complex conditions using the MODFLOW code (McDonald and Harbaugh, 1988) to determine groundwater flow.

Similarly to analytical models, numerical models have limitations in how they can be configured to match site conditions. Equations cannot describe all of the nuances for each term within the transport equation. That is, numerical models cannot exactly reproduce reality. However, compared to analytical models, numerical models can be configured to more closely match the site conditions and processes. There are also limitations in the type and quality/quantity of data that are available at any site to develop the coefficients necessary in the equations for the numerical model.

Stable isotope analysis: During the process of biological degradation, organisms often selectively metabolize molecules containing atoms of the most common isotope of an element. Isotopic fractionation occurs during biodegradation because bonds between different isotopes require different activation energy to react, and organisms will preferentially attack bonds of lower energy. While physical processes such as evaporation and dissolution can impact isotopic fractionation over the long-term, biological reactions can result in measurable isotopic fractionation over short time frames (Poulson and Drever 1999).

Recently, researchers have used stable isotope analysis to assess the extent of biodegradation relative to non-biological attenuation processes at affected sites (Lollar, Slater et al. 2001; Slater, Lollar et al. 2001). Stable isotope analyses have been performed for carbon, hydrogen and chlorine isotopes in organic compounds (Shouakar-Stash, Frape et al. 2003); however, carbon isotope ratios are determined most frequently (Ahad, Lollar et al. 2000; Hunkeler and Aravena 2000; Song, Conrad et al. 2002). In the case of carbon, organisms selectively target compounds containing ${ }^{12} \mathrm{C}$, the lighter carbon isotope, resulting in depletion of ${ }^{12} \mathrm{C}$-containing parent molecules and a relative enrichment of molecules containing ${ }^{13} \mathrm{C}$ (Slater, Lollar et al. 2001). At sites with active biodegradation, the carbon isotope signature in the downgradient area of the plume is significantly different (i.e. enriched in 13C) from the source area (Slater 2003). Carbon isotope signatures have been used as supporting evidence of intrinsic bioremediation for BTEX compounds as well as chlorinated ethenes (Lollar, Slater et al. 2001; Song, Conrad et al. 2002; Slater 2003). 


\subsection{Key Monitoring Concepts}

This part of the scenario describes groundwater monitoring programs that are appropriate for this particular scenario, and what scenario-specific monitoring is required. The scenario recommendations are based on the monitoring concept described by the Air Force's MAROS system (Aziz et al., 2000b).

The Air Force's MAROS system includes a methodology for determining an appropriate intensity level for long-term monitoring. As shown in the left column of Figure 21, the statistical trend in the source area (rows) and the statistical trend in the tail portion of the plume (columns) is used to indicate if a monitoring system should be (Figure 21):

- Extensive (E)

- Moderate (M)

- $\quad$ Limited (L)
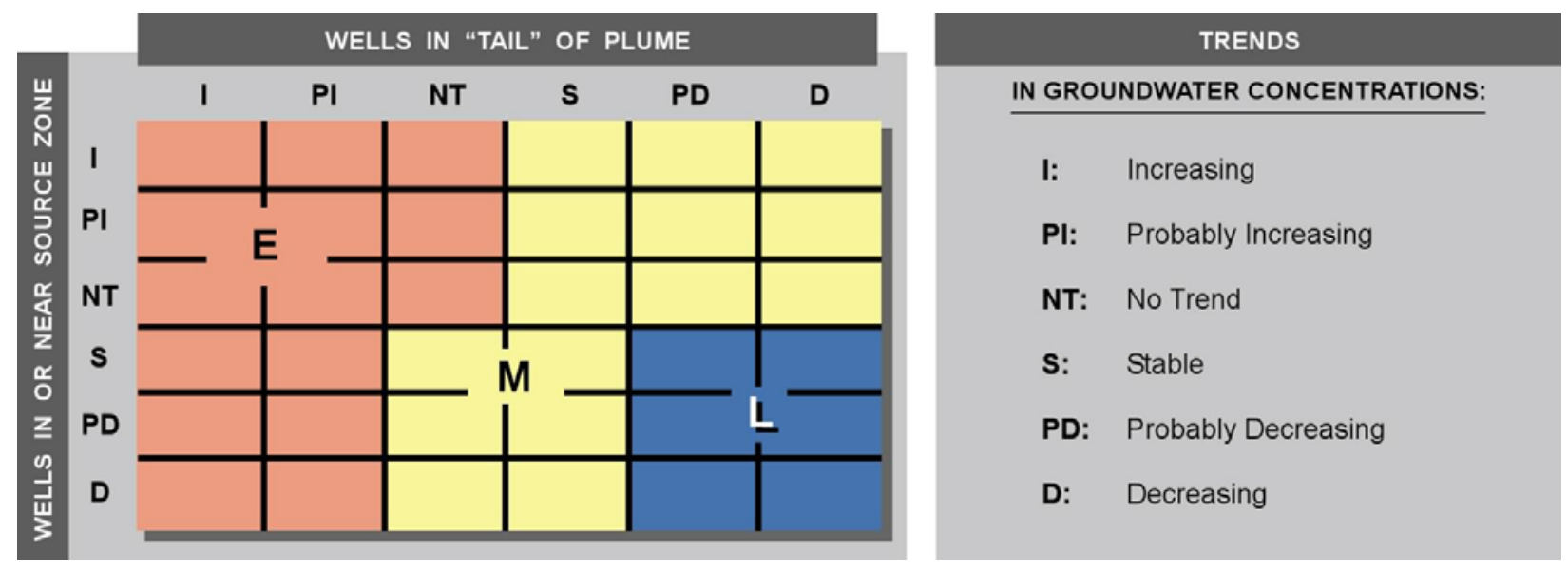

FIGURE 21. Monitoring Intensity Chart Based on Plume Stability Analysis for Wells in or Near the Subsurface Source ("source") and Wells in Plume Segment Downgradient of Source ("Tail") (Aziz et al., 2000). $\mathrm{E}=$ "Extensive" Monitoring System; $\mathrm{M}=$ "Moderate" Monitoring System; L = Limited Monitoring System.

The MAROS results are used together with the Time To Receptor (TTR) to indicate the frequency of monitoring (see Table 10). Note that the information in Table 10 presents very general guidelines for monitoring frequency, and more frequent or less frequent monitoring may be acceptable at many sites. 
TABLE 10. Relation of Time to Receptor and Monitoring System Category (Aziz et al., 2000b)

\begin{tabular}{lccc}
\hline TIME TO RECEPTOR (TTR) & \multicolumn{3}{c}{ Monitoring System Category } \\
\cline { 2 - 4 } Close (TTR $<2$ yrs) & Extensive & Moderate & Limited \\
\hline Medium $(2<$ TTR $<5$ yrs $)$ & Quarterly & $\begin{array}{l}\text { Biannually } \\
(6 \text { months })\end{array}$ & Annually \\
\hline Far (TTR $>5$ yrs $)$ & $\begin{array}{l}\text { Biannually } \\
(6 \text { months })\end{array}$ & Annually & Annually \\
TTR: time to receptor (distance to receptor/seepage velocity) & Annually & $\begin{array}{c}\text { Biennially } \\
\text { (2 year interval) }\end{array}$
\end{tabular}

Two types of monitoring may be prescribed in the scenario (Figure 22). The glide path (or transect) monitoring and sentry well monitoring each provide information that is needed to meet the monitoring requirements prescribed for MNA. Most plumes will need both types of monitoring, although, there may be exceptions for plumes that already have a preponderance of data indicating shrinkage of the plume.

\section{GLIDE PATH MONITORING $\quad$ DATA USE}

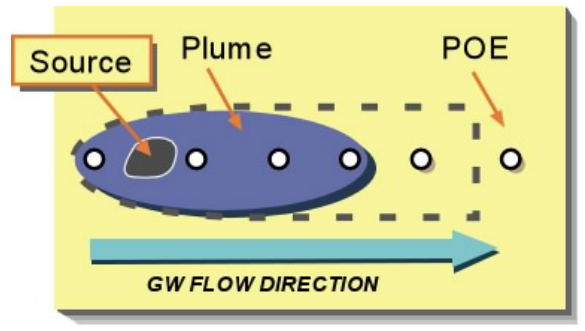

Plume monitoring along a transect parallel to plume migration to assess whether the trend of concentration of contaminants with distance continues to indicate that attenuation processes are working as expected.

SENTRY WELL MONITORING

DATA USE

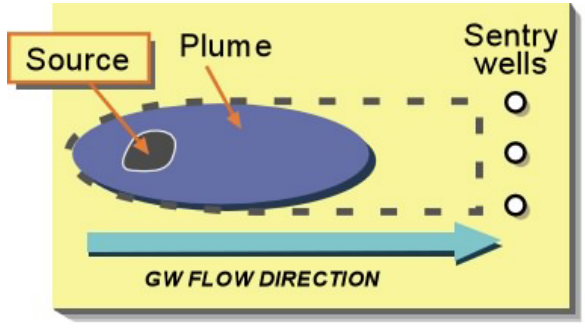

Final-line of defense to ensure that plume does not migrate beyond expected limits.

FIGURE 22. Two types of MNA monitoring.

\subsection{Key Uncertainty Concept}

This part of the scenario provides information on the key sources of uncertainty to consider for use of the scenario. Uncertainty must be considered as part of the MNA evaluation process. While there is not a quantitative uncertainty "value" that is necessary to meet in order to gain regulatory acceptance for MNA, it is important to understand the uncertainty associated with whether MNA will meet remediation goals as part of the final remedy selection process.

Uncertainty can refer to a number of different areas as part of an MNA evaluation (or for other remedies as well). Important categories of uncertainty to consider for MNA include the following. With each category, questions relating to the uncertainty in each category are also listed. 
- Conceptual model of the plume - Is the plume behavior and setting understood well enough to assess how the plume will change in the future?

- Site History and Source Definition - Is there sufficient information to define the mass of source, timeframe of the spill/contamination of the aquifer, and source flux? Can these quantities be bounded in a way that allows evaluation of MNA within likely bounds for source characteristics? Is there information about any other discharges that may have impacted the geochemistry at the site or activities affecting the hydrology?

- $\quad$ Plume definition - Are monitoring wells available to delineate the plume edges and define the progression of contaminant concentrations within the plume? Is the spacing between wells appropriate for the scale of the plume and hydrogeologic characteristics of the site? Is the vertical extent of the plume defined?

- Hydrogeology - Are the data for hydraulic gradient and hydraulic properties sufficient to describe the scale and heterogeneity of the hydrogeologic properties of the site? Do borehole logs define the stratigraphy and variation of stratigraphy with location? Are hydraulic head data available to determine the flow direction and any temporal/seasonal variations in hydrology? Are site specific data for hydraulic properties of the subsurface available?

- Attenuation processes - Are the attenuation processes clear from the geochemical data available? Are laboratory data available if field data do not adequately define the attenuation processes? Are there quantitative data to define the rate/extent of attenuation processes?

- Analysis - Is the analysis method suitable for the complexity of the site?

- Do calculations or simulations of plume fate and transport match available data?

- Are replicate measurements available to confirm field data values?

- Are sensitivity analyses, statistical analyses, or similar measures of uncertainty appropriate for evaluating the uncertainty of predictions for the future behavior of the plume?

\subsection{Key Data Analysis}

This part of the scenario lists the types of data analysis tasks (graphing, mass balance, modeling) that are useful to evaluate the scenario. Each scenario prescribes a certain combination of these different lines of evidence, based on the particular combination of modifying factors. Typical analysis methods are discussed below.

Note that some of these approaches are not appropriate if active treatment of the source or plume has been underway for an extended period. Graphical methods showing the historical change in concentrations will not be representative of MNA processes if active remediation processes are present at the site.

\section{Graphical and statistical analysis for mass loss:}

Concentration vs. time plots at individual wells: If it can be shown that concentrations are decreasing at a well and that this decrease is not due to the plume moving to other parts of the aquifer, this information is a direct indication of attenuation in the portion of the plume monitored by the well. The time period required to collect this type of data can be on the order of years for many plumes. This type of data would only be expected to show attenuation if the plume is old enough to have ceased migrating and is now shrinking. If a plume is relatively new, MNA may still be sufficient to stop the plume prior to reaching a receptor, but concentration vs. time data will likely not be a useful measure of plume attenuation because the plume may be either stable or still expanding. Depending on the relative change in parent and daughter products over time at the well, some information about the attenuation mechanism may be obtained, but it is not necessary to use this information as a line of evidence for attenuation.

Concentration vs. distance plots: For plumes where the migration patterns are well understood, concentration data at wells along a transect parallel with the groundwater flow direction can be used to demonstrate that attenuation is occurring along the plume flow path. It is very important with this type of analysis to ensure that any concentration differences along the flow path are not simply due to the fact that 
the plume is still evolving at the leading edge. Therefore, multiple concentration versus distance data between wells along a transect must be collected over a period of time to demonstrate that the concentration differences are due to attenuation and not due to plume migration characteristics. The time period required to collect this type of data can be on the order of years to tens of years for many plumes. Depending on the relative change in parent and daughter products over time along the axis of the plume, some information about the attenuation mechanism may be obtained and may be important to show that concentration changes along the plume are indeed due to contaminant transformation (e.g., observing a change in the relative amount of parent to daughter products).

Plume maps: Contaminant concentration data at wells within a plume for a specific point in time can be interpreted using concentration contouring techniques to produce a plume map. A series of plume maps showing how the extent and characteristics of the plume change over time can provide direct visual evidence for attenuation if the plume changes significantly enough to clearly show shrinkage or if the plume remains stable. Some plume characteristics such as the maximum observed concentration, plume area above selected concentration limits, and plume length can be calculated to augment the visual plume data. To use this type of analysis, the same concentration interpolation routine should be applied to generate plume maps at each time period. It is also important to consider any changes in the number or location of wells used in plume contouring. For instance, in some cases monitoring wells may be added to a plume over time. Later plume maps may, therefore, appear different than earlier maps if more data are used in the contouring process.

Mann-Kendall Trend Analysis: The Mann-Kendall test is a non-parametric statistical procedure that is well suited for analyzing trends in data over time (Gilbert, 1987). It does not require any assumptions as to the statistical distribution of the data (e.g. normal, log-normal, etc.) and can be used with data sets which include irregular sampling intervals and missing data. As a non-parametric test, it uses the ranking of temporal data over time to determine a trend. Aziz et al. (2003), extended the basis of the Mann-Kendall methodology to determine plume trends using Mann-Kendall " $S$ " statistic, the confidence factor, and the coefficient of variation to categorize temporal data into one of six categories: Increasing, Probably Increasing, Stable, Probably Decreasing, Decreasing, or No Trend. The U.S. Air Force Center for Environmental Excellence's MAROS software (Aziz et al, 2000b) uses this approach to categorize trends in individual monitoring wells, and then lumps results from groups of wells to define plume stability (see section 3.4).

A similar approach based on linear regression techniques is also used by the MAROS system to determine plume stability.

\section{Mass Balance:}

Mass balance is a simple accounting process that keeps track of loading (or inputs), attenuation, and the releases (or outputs). For MNA to be successful as a remedy, the attenuation impact on contaminant mass must sufficiently reduce the input of contaminant mass to acceptable levels prior to the plume reaching the defined receptor(s). There are several means of conducting a mass balance for a plume. Selection of the most appropriate approach is dependent on the type of data available and the general characteristics of the plume.

\section{Tool Kits}

There are several tools that are specifically designed to enable use of a mass balance calculation to quantify natural attenuation processes and evaluate the potential for MNA to meet remediation objectives. These tools include the BIOBALANCE software system ${ }^{1}$.

1 Developed by Groundwater Services (www.gsi-net.com), with the support of the Savannah River National Laboratory and DOE, to evaluate monitored natural attenuation at chlorinated solvent sites. 
Mass Flux Approach

An empirical approach to defining the mass balance can be applied if sufficient monitoring locations are available to define the mass flux at several positions along the migration pathway of the plume. Care should be used in this approach depending on the site properties and the age of the plume. Essentially, the plume must have reached steady-state concentrations at each mass flux monitoring location to use the empirical technique to assess the amount of attenuation between these locations. If contaminant concentrations are still changing with time at a downgradient monitoring location, transport processes in addition to attenuation processes are impacting the concentrations at this location and the mass flux technique is not appropriate. To apply the mass flux technique for MNA, mass fluxes at two or more locations are determined. The difference between the mass fluxes is equivalent to the attenuation occurring between these locations.

\section{Deterministic Approach}

Each component of the mass balance for a plume, the source loading (inputs), attenuation, and the releases (outputs) can be quantified independently and the mass balance calculated as a coupled fate and transport equation (either analytically or using numerical models). This approach depends on obtaining reasonable estimates for each of the processes that impact contaminant migration. Typically, it is necessary to use a sensitivity analysis or statistical analysis as part of calculating or simulating the fate and transport of a contaminant to assess the uncertainty in the estimates for the processes that impact contaminant migration. For plumes that are still expanding or where hydrologic conditions have or will change (e.g., terminating a pump-and-treat system), the deterministic approach may be necessary to evaluate MNA.

Modeling

A description of modeling is presented in section 5.4.

\subsection{Cost Considerations}

Cost for MNA is related to the effort required to evaluate MNA to the extent necessary to obtain approval for selection of MNA as a remedy and the continuing monitoring effort to demonstrate that MNA meets remediation goals. The site properties and plume characteristics impact the effort required, and therefore cost, of the evaluation and monitoring process. Some of the specific cost factors that are identified in the scenarios are discussed below.

\section{Site Hydrogeology}

Site hydrogeology impacts MNA cost either due to complexity or physical configuration of the subsurface. As hydrogeologic complexity increases, the number of monitoring/characterization locations needed to demonstrate MNA typically increase to provide enough information about the variation in plume conditions in areas with different hydrogeologic conditions. Hydrogeologic complexity may result from subsurface heterogeneity (e.g., multiple layers within an aquifer) or from non-uniform groundwater flow conditions (e.g., transient or multi-directional groundwater flow). Physically larger features such as thick vadose zones and thick aquifers may also increase costs because the cost for subsurface access increases with the depth of access required.

\section{Geochemistry}

Some important attenuation reactions are a function of site geochemistry. As discussed in section 5.1, there is more uncertainty in the type of reactions occurring at a site under the anoxic geochemical setting. Thus, more information may be needed to support an MNA remedy with anoxic site geochemistry. Variations in site geochemistry can also increase costs because more monitoring/characterization locations may be needed to determine the type of attenuation reactions occurring at the site.

\section{Plume Characteristics}

Larger plumes may require more monitoring/characterization locations than smaller plumes and have correspondingly higher costs. Other plume-related cost factors include 1) the stability of the plume where less stable plumes require more monitoring than stable or shrinking plumes and 2) the proximity of the 
plume to receptors where closer receptors likely require more monitoring locations and/or frequency to ensure no negative impacts.

A DNAPL source zone treatment cost study funded by SERDP compiled cost data from the peer reviewed literature, conference proceedings, state and federal government agency reports, internet databases, and a technical survey (McDade et al., 2004). The resulting data indicated that enhanced bioremediation has the lowest median cost per volume of $\$ 29 / \mathrm{yd}^{3}(\mathrm{n}=11)$; followed by thermal, chemical oxidation, and surfactant/cosolvent at $\$ 88 / \mathrm{yd}^{3}(\mathrm{n}=13), \$ 125 / \mathrm{yd}^{3}(\mathrm{n}=6)$, and $\$ 385 / \mathrm{yd}^{3}(\mathrm{n}=6)$, respectively. Only a weak correlation was observed between treatment size and total treatment cost. Longer treatment durations correlated to lower treatment costs per volume. Treatment performance appeared to be independent of unit treatment costs. The resulting cost statistics and unit costs can be used to compare the cost of source depletion projects against the life-cycle cost of long-term plume management techniques such as monitored natural attenuation or plume containment.

\subsection{Key Enhanced Attenuation Concepts}

This part of the scenario summarizes how enhanced attenuation (EA) can best be applied to manage this plume segment. Enhanced attenuation, in the context of application to augment MNA is categorized as those enhancements that are sustainable. A sustainable enhancement is an intervention action that continues until such time that the enhancement is no longer required to reduce contaminant concentrations or fluxes as part of an MNA-based remedy. There are two basic categories of EA listed below.

- Reduced Source Loading - If the flux of contaminants from the source is reduced, then existing natural attenuation processes may be better able to reduce contaminant concentrations to acceptable levels before the defined receptors. Sustainable enhancements in this category may include hydraulic manipulation such as diversion of surface water (e.g., surface caps or drains) or groundwater (e.g., slurry walls). Passive source reduction may also be a sustainable enhancement, for example, through injection of long lasting materials that provide diffusion barriers or promote degradation of the source (e.g., vegetable oil).

- Increased Attenuation Capacity - For some sites, a moderate increase in the attenuation capacity downgradient of the source may reduce contaminant concentrations sufficiently to meet remediation objectives. Either biological or abiotic enhancements that meet the criteria of being sustainable (e.g., long acting) may be appropriate. For instance, a permeable reactive barrier or phytoremediation may provide enough contaminant reduction in conjunction with natural attenuation processes to meet remediation goals. It may also be feasible to enhance attenuation near the discharge of the plume to protect receptors.

\section{SOURCE CONTROL VS. ENHANCED ATTENUATION}

This document draws a distinction between these two response actions. Source control is a onetime action for reducing the strength, size, and/or mass of the source zone. Enhanced attenuation is a response where an action is designed to make MNA sustainable over the long term. Early et al. (2006) provides additional information on Enhanced Attenuation.

Some remediation technologies (chemical oxidation, thermal treatment) clearly fit the definition of source control. In-situ biodegradation, however, can be either source control or enhanced biodegradation, depending on how it is applied. Enhance attenuation should be considered a "bridge" between source treatment and MNA.

\subsection{Key Source Control Concept}

Within the U.S. EPA "Use of Monitored Natural Attenuation at Superfund, RCRA Corrective Action, and Underground Storage Tank Sites" (OSWER Directive 9200.4-17P), the EPA states the expectation that "source control measures will be evaluated for all contaminated sites and that source control measures will be taken at most sites where practicable." As a guideline, the MNA Directive states the following: "Source control measures should use treatment to address "principal threat" wastes wherever practicable, and 
engineering controls such as containment for waste that poses a relatively low long-term threat or where treatment is impracticable." Additional guidance regarding contaminant sources is included in a listing of considerations for MNA as follows: "In determining whether MNA is an appropriate remedy for soil or groundwater at a given site, EPA or other regulatory authorities should consider the following: "...The nature and distribution of sources of contamination and whether these sources have been, or can be, adequately controlled."

Based on the MNA Directive, evaluating source control is an integral part of evaluating MNA at a site. The impact of the source flux and potential mitigation of this contaminant flux are part of the evaluation processes described above. In particular, use of a mass balance approach includes explicit consideration of source flux. Depending on the impact of the source flux on the ability of MNA to meet remediation goals, source control measures may be needed in some cases to supplement MNA. The characteristic of each scenario determine the likely relative importance of source control measures. Thus, each scenario includes considerations for source control based on the scenario and modifying factors that have been identified for the scenario. Source control in the context of scenarios includes active measures such as chemical oxidation, thermal treatment, and active containment. In contrast, Section 5.9 discusses how more passive source control techniques may be considered as part of an Enhanced Attenuation approach.

Source Control Background:

The performance of 59 source treatment projects at chlorinated solvent sites was evaluated by McGuire et al., 2004 as part of a SERDP DNAPL source zone initiative. The four technologies included in the study are chemical oxidation, enhanced bioremediation, thermal treatment, and surfactant/cosolvent flushing. Performance was evaluated by examining temporal groundwater concentration data before and after source depletion was performed.

Results indicated that all four technologies have median concentration reductions of $88 \%$ or greater for the parent chlorinated volatile organic compound (CVOC). Approximately $75 \%$ of the source depletion projects were able to achieve a $70 \%$ reduction in parent compound concentrations. A median reduction in total CVOC concentrations (parent plus daughter compounds) of $72 \%$ was observed at 12 chemical oxidation sites and $62 \%$ at 21 enhanced bioremediation sites.

Simple planning-level models developed by Falta (Falta et al., 2005a,b) suggest that source treatment projects do not result in a linear relationship between steady-state plume length and mass of source removed in most cases. Rather a more logarithmic relationship should be expected at many sites (i.e., the percentage reduction in steady-state plume length is proportional to the log of the percentage reduction in source mass). A similar relationship was presented by Newell and Adamson (2005) who indicated a nonlinear relationship between the amount of mass remaining following source depletion and the reduction in the remediation timeframe using planning-level source decay models. 


\subsection{REFERENCES}

\section{Numbered References for Figures 8-19 (for General References see page 50)}

1. Bagley DM and JM Gossett. 1995. Chloroform Degradation in Methanogenic Methanol Enrichment Cultures and by Methanosarcina Barkeri 227. Appl. Environ. Microbiol. 61(9):3195-3201.

2. Bouwer EJ and PL McCarty. 1983. Transformations of 1- and 2-Carbon Halogenated Aliphatic Organic Compounds under Methanogenic Conditions. Appl. Environ. Microbiol. 45(4):1286-1294.

3. Bouwer EJ and PL McCarty. 1983. Transformations of Halogenated Organic Compounds under Denitrification Conditions. Appl. Environ. Microbiol. 45(4):1295-1299.

4. Bradley, P.M. and F.H. Chappelle. 1997. Kinetics of DCE and VC mineralization under methanogenic and Fe(III)-reducing conditions. Environ. Sci. Technol. 31(9):2692-2696.

5. Bradley, P.M. and F.H. Chapelle. 2000. Aerobic microbial mineralization of dichloroethene as sole carbon substrate. Environ. Sci. Technol., 34(1):221-223.

6. Chang, H. and L. Alvarez-Cohen. 1996. Biodegradation of individual and multiple chlorinated aliphatic hydrocarbons by methane-oxidizing cultures. Appl. Environ. Microbiol. 62:3371-3377.

7. Chen, C., J.A. Puhakka, and J.F. Ferguson. 1996. Transfromations of 1,1,2,2,-tetrachloroethane under methanogenic conditions. Environ. Sci. Technol. 30(2):542-547.

8. Culubret, E.N., M. Luz, R. Amils, and J.L. Sanz. 2001. Biodegradation of 1,1,1,2-tetrachloroethane under methanogenic conditions. Water Sci. Technol. 44(4):117-122.

9. DeBruin, W. P., M. J. J. Kotterman, M. A. Posthumus, G. Schraa, and A. J. B. Zehnder. 1992. Complete biological reductive transformation of tetrachloroethene to ethane. Appl. Environ. Microbiol. 58:1996-2000.

10. Egli C, S Stromeyer, AM Cook, and T Leisinger. 1990. Transformation of tetra- and trichloromethane to $\mathrm{CO}_{2}$ by anaerobic bacteria is a non-enzymatic process. FEMS Microbiology Letters 68:207-212.

11. Freedman, D. L. and J. M. Gossett. 1989. Biological reductive dechlorination of tetrachloroethylene and trichloroethylene to ethylene under methanogenic conditions. Appl. Environ. Microbiol. :21442151.

12. Freedman DL and JM Gossett. 1991. Biodegradation of Dichloromethane and Its Utilization as a Growth Substrate under Methanogenic Conditions. Appl. Environ. Microbiol. 57(10):2847-2857.

13. Freedman, D.L., M. Swamy, N.C. Bell, and M.F. Verce. 2004. Biodegradation of chloromethane by Pseudomonas aeruginosa strain NB1 under nitrate-reducing and aerobic conditions. Appl. Environ. Microbiol. 70(8):4629-4634.

14. Haag, W.R. and T. Mill. 1988. Effect of a subsurface sediment on hydrolysis of haloalkanes and epoxides. Environ. Sci. Technol. 22:658-663.

15. Hartmans, S. and J.A.M. de Bont. 1992. Aerobic vinyl chloride metabolism in Mycobacterium aurum L1. Appl. Environ. Microbiol. 58:1220-1226. 


\section{Numbered References for Figures 8-19 (for General References see page 50)}

16. Hunkeler, D. and R. Aravena. 2000. Evidence of substantial carbon isotope fractionation among substrate, inorganic carbon, and biomass during aerobic mineralization of 1,2-dichloroethane by Xanthobacter autotrophicus. Appl. Environ. Microbiol. 66:4870-4876.

17. Jeffers P.M., L.M. Ward, L.M. Woytowitch, and N.L. Wolfe. 1989. Homogeneous hydrolysis rate constants for selected chlorinated methanes, ethanes, ethenes, and propanes. Environ. Sci. Technol. 23(8):965-967.

18. Kim, Y., D.J. Arp and L. Semprini. 2000. Chlorinated solvent cometabolism by butane-grown mixed culture. J. Environmental Engineering. 126(10):934-942.

19. Kim, Y., D.J. Arp and L. Semprini. 2002. Kinetic and inhibition studies for the aerobic cometabolism of 1,1,1-trichloroethane, 1,1-dichloroethylene, and 1,1-dichloroethane by a butane-grown mixed culture. Biotechnol. Bioengineering. 80(5):498-508.

20. Klier, N.J., R.J. West, P.A. Donberg. 1998. Aerobic biodegradation of dichloroethylenes in surface and subsurface soils. Chemosphere., 38(5):1175-1188.

21. Leisinger, T., R. Bader, R. Hermann, M. Schmid-Appert, and S. Vuilleumier. 1994. Microbes, enzymes and genes involved in dichloromethane utilization. Biodegradation. 5(3-4):237-248.

22. Mabey W and T Mill. 1978. Critical Review of Hydrolysis of Organic Compounds in Water under Environmental Conditions. J. Phys. Chem. Ref. Data. 7(2):383-415.

23. Pagan, M., W.J. Cooper, and J.A. Joens. 1998. Kinetic studies of the homogeneous abiotic reactions of several chlorinated aliphatic compounds in aqueous solution, Appl. Geochemistry 13(6):779785.

24. Vannelli T, A Studer, M Kertesz, and T Leisinger. 1998. Chloromethane Metabolism by Methylobacterium sp. Strain CM4. Appl. Environ. Microbiol. 64(5)1933-1936.

25. Vogel, T.M. and P.L. McCarty. 1987a. Abiotic and biotic transformation of 1,1,1-trichloroethane under methanogenic conditions. Environ. Sci. Technol. 21(12):1208-1213. 


\section{General References}

Ahad, J. M. E., B. S. Lollar, E. A. Edwards, G. F. Slater and B. Sleep, 2000. "Carbon isotope fractionation during anaerobic biodegradation of toluene: implications for intrinsic bioremediation", Environmental Science and Technology 34(5): 892-896.

Aller, L., Bennet, T., Lehr, J.H. and Petty, R.J., 1987. DRASTIC: a standardized system for evaluating groundwater pollution potential using hydrogeologic settings, U.S. EPA Report 600/2-85/018.

ASTM, 2004. Standard Guide for Risk-Based Corrective Action, E2081-00(2004)e1, American Society for Testing and Materials, West Conshohocken, Pennsylvania.

Aziz, C.E., C.J. Newell, J.R. Gonzales, P.E. Haas, T.P. Clement, and Y. Sun, 2000a. BIOCHLOR Natural Attenuation Decision Support System, User's Manual Version 1.0, U.S. EPA, Office of Research and Development, EPA/600/R-00/008, Washington D.C., January 2000, www.gsi-net.com

Aziz, J.J., C.J. Newell, H.S. Rifai, M. Ling, and J.R. Gonzales, 2000b. Monitoring and Remediation Optimization System (MAROS) Software User's Guide, October 16, 2000. www.gsi-net.com

Aziz, J.J.; M. Ling, H.S. Rifai, C.J. Newell, and J.R. Gonzales, 2003. "MAROS: a Decision Support System for Optimizing Monitoring Plans", Journal of Ground Water, Vol. 41, No. 3.

Chapelle, F.H. M.A. Widdowson, J. S. Brauner, E. Mendez III, and C.C. Casey, 2003. Methodology for Estimating Times of Remediation Associated with Monitored Natural Attenuation, U.S. Geological Survey, Water-Resources Investigations Report 03 - 4057.

Clement, T.P. 1997. RT3D - A Modular Computer Code for Simulating Reactive Multi-Species Transport in 3-Dimensional Groundwater Aquifers . Pacific Northwest National Laboratory, Richland, WA, USA. PNNL-11720. Found online at: http://bioprocess.pnl.gov/rt3d.htm .

Clement, T.P. and N.L. Jones. 1998. RT3D Tutorials for GMS Users . Pacific Northwest National Laboratory, Richland, Washington, USA. PNNL-11805. Found online at: http://bioprocess.pnl.gov/rt3d.htm .

Early, T., B. Borden, M. Heitkamp, B.B. Looney, D. Major, J. Waugh, G. Wein, T. Wiedemeier, K.M. Vangelas, K.M. Adams, and C.H. Sink. 2006. Enhanced Attenuation: Approaches to Increase the Natural Treatment Capacity of a System. WSRC-TR-2005-00198. Washington Savannah River Company, Aiken, SC, USA. Found online at http://www.osti.gov.

Falta, R.W., P.S. Rao, and N. Basu. 2005a. "Assessing the impacts of Partial Mass Depletion in DNAPL Source Zones: I. Analytical Modeling of Source Strength Functions and Plume Response", Journal of Contaminant Hydrology, Volume 78, Issue 4, August 2005, Pages 259-280.

Falta, R.W., N. Basu, and P.S. Rao. 2005b. "Assessing the impacts of Partial Mass Depletion in DNAPL Source Zones: II. Coupling Source Strength Functions to Plume Evolution", Journal of Contaminant Hydrology, Volume 79, Issues 1-2, September 2005, Pages 45-66.

Gilbert R O. 1987. Statistical methods for environmental pollution monitoring, New York: Van Nostrand Reinhold Co. 320pp.

Hunkeler, D. and R. Aravena, 2000. "Determination of compound-specific carbon isotope ratios of chlorinated methanes, ethanes, and ethenes in aqueous samples", Environmental Science and Technology 34(13): 2839-2844.

Johnson, P., P. Lundegard, J. Catts, K. DiSimone, D. Eley, and K. Schroeder, 2003. Source zone natural attenuation field measurements, data interpretation and data reduction at the former Guadalupe Oil Field (Version 2.1), Dept. of Civil and Environmental Engineering, Arizona State University, Tempe, Arizona.

Lollar, B. S., G. F. Slater, B. Sleep, M. Witt, G. M. Klecka, M. Harkness and J. Spivack, 2001. "Stable Carbon Isotope Evidence for Intrinsic bioremediation of tetrachloroethene and trichloroethene at Area 6, Dover Air Force Base", Environmental Science and Technology 35(2): 261-269.

McDade, J.M., McGuire, T.M., and C.J. Newell, 2005. "Analysis of DNAPL Source Depletion Costs at 36 Field Sites", Remediation, 2005, 15(2). 
McDonald, M.G., and Harbaugh, A.W., 1988, A modular three- dimensional finite-difference ground-water flow model: U.S. Geological Survey Techniques of Water-Resources Investigations, book 6, chap. A1, $586 \mathrm{p}$.

McGuire, T.M., J.M. McDade, and C.J. Newell, 2006. "Performance of DNAPL Source Depletion Technologies at 59 Chlorinated Solvent-Impact Sites", Groundwater Monitoring and Remediation, Vol 26, No. 1, pg 73-84.

McGuire, T.M., C.J. Newell, B.B. Looney, K.M. Vangelas, 2003. Historical and Retrospective Survey of Monitored Natural Attenuation: A Line of Inquiry Supporting Monitored Natural Attenuation and Enhanced Passive Remediation of Chlorinated Solvents, Westinghouse Savannah River Company, Department of Energy, Aiken, South Carolina.

McGuire, T.M., C.J. Newell, B.B. Looney, K.M. Vangelas, and C.H. Sink, 2004. "Historical Analysis of Monitored Natural Attenuation: A Survey of 101 Chlorinated Solvent Sites and 45 Solvent Plumes", Remediation, Winter, 2004.

National Research Council, 2000. Natural Attenuation for Groundwater Remediation, National Academy Press, Washington, D.C.

Newell, C.J. and D.T. Adamson, 2005. "Planning-Level Source Decay Models to Evaluate Impact of Source Depletion on Remediation Timeframe", Remediation, in press.

Newell, C.J. and C.E. Aziz, 2004. "Long-Term Sustainability of Reductive Dechlorination Reactions at Chlorinated Solvents Sites", Biodegradation, 15: 387-394, 2004.

Newell, C. J., L. P. Hopkins, and P. B. Bedient, 1990. "A Hydrogeologic Database for Groundwater Modeling", Ground Water, Vol. 28, No. 5.

Poulson, S. R. and J. I. Drever, 1999. "Stable Isotope (C, Cl and H) fractionation during vaporization of trichloroethylene", Environmental Science and Technology 33(20): 3689-3694.

Shouakar-Stash, O., S. K. Frape and R. J. Drimmie, 2003. "Stable hydrogen, carbon and chlorine isotope measurements of selected chlorinated organic solvents", Journal of Contaminant Hydrology 60: 211-228.

Slater, G. F. (2003). "Stable isotope forensics--When isotopes work", Environmental Forensics 4: 13-23.

Slater, G. F., B. S. Lollar, B. E. Sleep and E. A. Edwards, 2001. "Variability in Carbon Isotopic Fractionation during Biodegradation of Chlorinated Ethenes: Implications for Field Applications", Environmental Science and Technology 35(5): 901-907.

Song, D. L., M. E. Conrad, K. S. Sorenson and L. Alvarez-Cohen,2002. "Stable carbon isotope fractionation during enhanced In Situ bioremediation of trichloroethene", Environmental Science and Technology 36(10): 2262-2268.

U.S. Environmental Protection Agency, 1999. Use of Monitored Natural Attenuation at Superfund, RCRA Corrective Action, and Underground Storage Tank Site, Office of Solid Waste and Emergency Response (OSWER), Directive 9200.4-17P, Final Draft, Washington, D.C., April 21, 1999.

U.S. Environmental Protection Agency, 1998. "Technical Protocol for Evaluation Natural Attenuation of Chlorinated Solvents in Groundwater," Wiedemeier, T. H., M.A. Swanson, D.E. Moutoux, E.K. Gordon, J.T. Wilson, B.H. Wilson, D.H. Kampbell, J.E. Hansen, and P. Haas, F. Chapelle, U.S. Environmental Protection Agency, EPA/600R0128, Sept. 1998.

U.S. Environmental Protection Agency, 2002. Calculation and Use of First-Order Rate Constants For Monitored Natural Attenuation Studies, Newell, C.J., H.S. Rifai, J.T. Wilson, J.A. Connor, and J.J. Aziz, M.P. Suarez, U.S. EPA Remedial Technology Fact Sheet, U.S. Environmental Protection Agency. EPA/540/S-02/500, November 2002. www.epa.gov/ada/pubs/issue.html 


\section{APPENDIX 1: SELECTING HYDROGEOLOGIC SETTING FROM DRASTIC SETTINGS}

\section{Instructions:}

Use the U.S. EPA's DRASTIC System (U.S. EPA, 1987, EPA-600/2-87-035) to select a DRASTIC Hydrogeologic Setting that best corresponds to your site. (Note the DRASTIC Hydrogeologic Settings are grouped by Hydrogeologic Region (Column 2 below). Find the DRASTIC Hydrogeologic Setting Name in Column 3, and then determine the Scenarios Hydrogeologic Setting in Column 4.

\begin{tabular}{|c|c|c|c|c|}
\hline $\begin{array}{c}\text { Column } 1 \\
\text { DRASTIC } \\
\text { No } \\
\end{array}$ & $\begin{array}{c}\text { Column } 2 \\
\text { DRASTIC Hydrogeologic Region }\end{array}$ & $\begin{array}{c}\text { Column } 3 \\
\text { DRASTIC Hydrogeologic Setting Name }\end{array}$ & $\begin{array}{c}\text { Column } 4 \\
\text { Scenarios Hydrogeologic } \\
\text { Scenario }\end{array}$ & Comments \\
\hline $1 \mathrm{Aa}$ & Western Mountain Ranges & Mountain Slopes -East & $\mathrm{H} 5$ & Metamorphic /lgneous rock \\
\hline $1 \mathrm{Ab}$ & Western Mountain Ranges & Mountain Slopes -West & $\mathrm{H} 5$ & Metamorphic /lgneous rock \\
\hline 1Ba & Western Mountain Ranges & Alluvial Mountain Valleys- East & $\mathrm{H} 2$ & Sand Gravel,K=100-300 \\
\hline $1 \mathrm{Bb}$ & Western Mountain Ranges & Alluvial Mountain Valleys - West & $\mathrm{H} 2$ & Sand Gravel,K=100-300 \\
\hline $1 \mathrm{Ca}$ & Western Mountain Ranges & Mountain Flanks -East & $\mathrm{H} 4$ & SS, LS, SH,K=100-300 \\
\hline $1 \mathrm{Cb}$ & Western Mountain Ranges & Mountain Flanks -West & $\mathrm{H} 4$ & SS, LS, SH,K=100-300 \\
\hline 1D & Western Mountain Ranges & Glaciated Mountain Valleys & $\mathrm{H} 1$ & \\
\hline $1 \mathrm{~Eb}$ & Western Mountain Ranges & Wide Alluvial Valleys ( External Drainage) -East & $\mathrm{H} 1$ & \\
\hline $1 \mathrm{Ea}$ & Western Mountain Ranges & Wide Alluvial Valleys ( External Drainage) -West & $\mathrm{H} 1$ & \\
\hline $1 \mathrm{~F}$ & Western Mountain Ranges & Coastal Beaches & $\mathrm{H} 1$ & \\
\hline $1 G$ & Western Mountain Ranges & Swamp/Marsh & $\mathrm{H} 4$ & \\
\hline $1 \mathrm{H}$ & Western Mountain Ranges & Mud Flows & $\mathrm{H} 1$ & \\
\hline $2 \mathrm{~A}$ & Alluvial Basins & Mountain Slopes & $\mathrm{H} 5$ & \\
\hline $2 B$ & Alluvial Basins & Alluvial Mountain Valleys & $\mathrm{H} 1$ & \\
\hline $2 \mathrm{C}$ & Alluvial Basins & Alluvial Fans & $\mathrm{H} 1$ & \\
\hline $2 \mathrm{D}$ & Alluvial Basins & Alluvial Basins (Internal Drainage) & $\mathrm{H} 1$ & \\
\hline $2 \mathrm{E}$ & Alluvial Basins & Playa Lakes & $\mathrm{H} 1$ & \\
\hline $2 \mathrm{~F}$ & Alluvial Basins & Swamp/Marsh & $\mathrm{H} 4$ & \\
\hline $2 \mathrm{G}$ & Alluvial Basins & Coastal Lowlands & $\mathrm{H} 1$ & \\
\hline $2 \mathrm{Ha}$ & Alluvial Basins & River Alluvium without Overbank Deposits & $\mathrm{H} 1$ & \\
\hline 21 & Alluvial Basins & Mud Flows & $\mathrm{H} 1$ & \\
\hline $2 \mathrm{~J}$ & Alluvial Basins & Alternating SandStone and Shale Sequence & $\mathrm{H} 4$ & \\
\hline $2 \mathrm{~K}$ & Alluvial Basins & Continental Depoits & $\mathrm{H} 1$ & \\
\hline $3 A$ & Columbia Lava Plateau & Mountain Slopes & H5 & \\
\hline 3B & Columbia Lava Plateau & Alluvial Mountain Valleys & $\mathrm{H} 1$ & \\
\hline $3 C$ & Columbia Lava Plateau & Hydraulically Connected Lava Flows & H5 & \\
\hline $3 \mathrm{D}$ & Columbia Lava Plateau & Lava Flows Not Connected Hydraulically & $\mathrm{H} 4$ & \\
\hline $3 \mathrm{E}$ & Columbia Lava Plateau & Alluvial Fans & $\mathrm{H} 1$ & \\
\hline $3 \mathrm{~F}$ & Columbia Lava Plateau & Swamp/Marsh & H5 & \\
\hline $3 G$ & Columbia Lava Plateau & River Alluvium & $\mathrm{H} 1$ & \\
\hline $4 \mathrm{~A}$ & Colorado Plateau and Wyoming Basin & Resistant Ridges & $\mathrm{H} 4$ & \\
\hline 4B & Colorado Plateau and Wyoming Basin & Consolidated Sedimentary Rocks & H5 & \\
\hline $4 \mathrm{C}$ & Colorado Plateau and Wyoming Basin & River Alluvium & $\mathrm{H} 1$ & \\
\hline $4 \mathrm{D}$ & Colorado Plateau and Wyoming Basin & Alluvium and Dune Sand & $\mathrm{H} 2$ & \\
\hline $4 \mathrm{E}$ & Colorado Plateau and Wyoming Basin & Swamp/Marsh & $\mathrm{H} 1$ & \\
\hline $5 \mathrm{~A}$ & High Plains & Ogallala & $\mathrm{H} 1$ & \\
\hline $5 B$ & High Plains & Alluvium & $\mathrm{H} 1$ & \\
\hline $5 \mathrm{C}$ & High Plains & Sand Dune & $\mathrm{H} 1$ & \\
\hline $5 \mathrm{D}$ & High Plains & Playa Lakes & $\mathrm{H} 1$ & \\
\hline $5 \mathrm{E}$ & High Plains & Braided River Deposits & $\mathrm{H} 1$ & \\
\hline $5 \mathrm{~F}$ & High Plains & Swamp/Marsh & $\mathrm{H} 1$ & \\
\hline $5 \mathrm{Ga}$ & High Plains & River Alluvium With OverBank Deposits & $\mathrm{H} 1$ & \\
\hline $5 \mathrm{~Gb}$ & High Plains & River Alluvium without Overbank Deposits & $\mathrm{H} 1$ & \\
\hline $5 \mathrm{H}$ & High Plains & $\begin{array}{c}\text { Alternating SandStone Lime stone and Shale } \\
\text { Sequence }\end{array}$ & $\mathrm{H} 4$ & \\
\hline $6 \mathrm{~A}$ & Non Glaciated Central & Mountain Slopes & $\mathrm{H} 4$ & \\
\hline $6 \mathrm{~B}$ & Non Glaciated Central & Alluvial Mountain Valleys & $\mathrm{H} 1$ & \\
\hline $6 \mathrm{C}$ & Non Glaciated Central & Mountain Flanks & $\mathrm{H} 4$ & \\
\hline $6 \mathrm{Da}$ & Non Glaciated Central & $\begin{array}{c}\text { Alternating Sand Stone, LimeStone and Shale- } \\
\text { Thin Soil }\end{array}$ & $\mathrm{H} 4$ & \\
\hline $6 \mathrm{Db}$ & Non Glaciated Central & $\begin{array}{c}\text { Alternating Sand Stone, LimeStone and Shale- } \\
\text { Deep Regolith }\end{array}$ & $\mathrm{H} 4$ & \\
\hline
\end{tabular}

Note: ${ }^{* *}$ For finding the scenarios corresponding to given Levels, refer USER'S MANUAL "Chlorinated Solvent Plume" Scenarios. 
WSRC-STI-2006-00096, Rev. 1

Occtober 2, 2006

Page A-2 of A-2

\begin{tabular}{|c|c|c|c|c|}
\hline $\begin{array}{l}\text { Column } 1 \\
\text { DRASTIC } \\
\text { No }\end{array}$ & $\begin{array}{c}\text { Column } 2 \\
\text { DRASTIC Hydrogeologic Region }\end{array}$ & $\begin{array}{c}\text { Column } 3 \\
\text { DRASTIC Hydrogeologic Setting Name }\end{array}$ & $\begin{array}{c}\text { Column } 4 \\
\text { Scenarios Hydrogeologic } \\
\text { Scenario }\end{array}$ & Comments \\
\hline $6 \mathrm{E}$ & Non Glaciated Central & Solution Limestone & H5 & $\begin{array}{c}\text { karst limestone so took it as } \\
\text { fractured }\end{array}$ \\
\hline $6 \mathrm{Fa}$ & Non Glaciated Central & River Alluvium With OverBank Deposits & $\mathrm{H} 1$ & \\
\hline $6 \mathrm{Fb}$ & Non Glaciated Central & River Alluvium Without OverBank Deposits & $\mathrm{H} 1$ & \\
\hline $6 \mathrm{G}$ & Non Glaciated Central & Braided River Deposits & $\mathrm{H} 1$ & \\
\hline $6 \mathrm{H}$ & Non Glaciated Central & Triassic Basins & $\mathrm{H} 5$ & Massive sand stone \\
\hline 61 & Non Glaciated Central & Swamp/Marsh & $\mathrm{H} 4$ & \\
\hline $6 \mathrm{~J}$ & Non Glaciated Central & Metamorphic/lgneous Domes and Fault Blocks & H5 & \\
\hline $6 \mathrm{~K}$ & Non Glaciated Central & Unconsolidated and Semi-Consolidated Aquifers & $\mathrm{H} 1$ & \\
\hline $7 \mathrm{Aa}$ & Glaciated Central & Glacial Till Over Bedded Sedimentary Rocks & $\mathrm{H} 4$ & \\
\hline $7 A b$ & Glaciated Central & Glacial Till Over Outwash & $\mathrm{H} 1$ & \\
\hline $7 A c$ & Glaciated Central & Glacial Till Over Solution Limestone & H5 & \\
\hline 7Ad & Glaciated Central & Glacial Till Over Sandstone & H5 & Massive sand stone \\
\hline $7 \mathrm{Ae}$ & Glaciated Central & Glacial Till Over Shale & $\mathrm{H} 2$ & Massive Shale \\
\hline 7Ba & Glaciated Central & Outwash & $\mathrm{H} 1$ & \\
\hline $7 \mathrm{Bb}$ & Glaciated Central & Outwash Over Bedded Sedimentary Rock & $\mathrm{H} 4$ & \\
\hline 7Bc & Glaciated Central & Outwash Over Solution Limestone & H5 & \\
\hline $7 \mathrm{C}$ & Glaciated Central & Moraine & $\mathrm{H} 1$ & \\
\hline 7D & Glaciated Central & Buried Valley & $\mathrm{H} 1$ & \\
\hline 7Ea & Glaciated Central & River Alluvium without Overbank Deposits & $\mathrm{H} 1$ & \\
\hline $7 \mathrm{~F}$ & Glaciated Central & Glacial Lake Deposits & $\mathrm{H} 4$ & \\
\hline $7 G$ & Glaciated Central & Thin Till Over Bedded Sedimentary Rock & $\mathrm{H} 4$ & \\
\hline $7 \mathrm{H}$ & Glaciated Central & Beaches, Beach Ridges and Sand Dunes & $\mathrm{H} 1$ & \\
\hline 71 & Glaciated Central & Swamp/Marsh & $\mathrm{H} 1$ & \\
\hline $8 \mathrm{~A}$ & Piedmont And Blue Ridge & Mountain Slopes & $\mathrm{H} 5$ & \\
\hline $8 \mathrm{~B}$ & Piedmont And Blue Ridge & Alluvial Mountain Valleys & $\mathrm{H} 1$ & \\
\hline $8 \mathrm{C}$ & Piedmont And Blue Ridge & Mountain Flanks & $\mathrm{H} 4$ & \\
\hline $8 \mathrm{D}$ & Piedmont And Blue Ridge & Regolith & H5 & \\
\hline $8 \mathrm{E}$ & Piedmont And Blue Ridge & River Alluvium & $\mathrm{H} 1$ & \\
\hline $8 \mathrm{~F}$ & Piedmont And Blue Ridge & Mountain Crests & $\mathrm{H} 5$ & \\
\hline $8 G$ & Piedmont And Blue Ridge & Swamp/Marsh & H5 & \\
\hline $9 \mathrm{~A}$ & Northeast and Superior Uplands & Mountain Slopes & $\mathrm{H} 5$ & \\
\hline $9 \mathrm{~B}$ & Northeast and Superior Uplands & Alluvial Mountain Valleys & $\mathrm{H} 1$ & \\
\hline $9 \mathrm{C}$ & Northeast and Superior Uplands & Mountain Flanks & $\mathrm{H} 4$ & \\
\hline 9Da & Northeast and Superior Uplands & Glacial Till Over Crystalline Bedrock & H5 & \\
\hline $9 \mathrm{Db}$ & Northeast and Superior Uplands & Glacial Till Over Outwash & $\mathrm{H} 1$ & \\
\hline $9 \mathrm{E}$ & Northeast and Superior Uplands & Outwash & $\mathrm{H} 1$ & \\
\hline $9 \mathrm{~F}$ & Northeast and Superior Uplands & Moraine & $\mathrm{H} 1$ & \\
\hline $9 \mathrm{Ga}$ & Northeast and Superior Uplands & River Alluvium With OverBank Deposits & $\mathrm{H} 1$ & \\
\hline $9 \mathrm{~Gb}$ & Northeast and Superior Uplands & River Alluvium Without Overbank Deposits & $\mathrm{H} 1$ & \\
\hline $9 \mathrm{H}$ & Northeast and Superior Uplands & Swamp/Marsh & $\mathrm{H} 5$ & \\
\hline 91 & Northeast and Superior Uplands & Bedrock Uplands & H5 & \\
\hline $9 \mathrm{~J}$ & Northeast and Superior Uplands & Glacial Lake/Glacial Marine Deposits & $\mathrm{H} 5$ & \\
\hline $\begin{array}{c}9 \mathrm{~K} \\
10 \mathrm{Aa}\end{array}$ & $\begin{array}{l}\text { Northeast and Superior Uplands } \\
\text { Atlantic and Gulf Coastal Plain }\end{array}$ & $\begin{array}{c}\text { Beaches, Beach Ridges and Sand Dunes } \\
\text { Regional Aquifer }\end{array}$ & $\begin{array}{l}\mathrm{H} 5 \\
\mathrm{H} 1\end{array}$ & \\
\hline $10 \mathrm{Ab}$ & Atlantic and Gulf Coastal Plain & $\begin{array}{c}\text { Unconsolidated and Semi-Consolidated Shallow } \\
\text { Surficial Aquifers }\end{array}$ & $\mathrm{H} 1$ & \\
\hline $10 \mathrm{Ba}$ & Atlantic and Gulf Coastal Plain & River Alluvium With OverBank Deposits & $\mathrm{H} 1$ & \\
\hline $10 \mathrm{Bb}$ & Atlantic and Gulf Coastal Plain & River Alluvium Without Overbank Deposits & $\mathrm{H} 1$ & \\
\hline $10 \mathrm{C}$ & Atlantic and Gulf Coastal Plain & Swamp & $\mathrm{H} 1$ & \\
\hline $11 \mathrm{~A}$ & Southeast Coastal Plain Ground-Water Region & $\begin{array}{c}\text { Solution Limestone and Shallow Surficial } \\
\text { Aquifers }\end{array}$ & H5 & \\
\hline 11B & Southeast Coastal Plain Ground-Water Region & Coastal Deposits & $\mathrm{H} 1$ & \\
\hline $11 \mathrm{C}$ & Southeast Coastal Plain Ground-Water Region & Swamp & H5 & \\
\hline 11D & Southeast Coastal Plain Ground-Water Region & Beaches and Bars & $\mathrm{H} 1$ & \\
\hline $12 \mathrm{~A}$ & Hawaiian islands Ground-water region & Mountain Slopes & $\mathrm{H} 5$ & Basalt \\
\hline $12 B$ & Hawaiian islands Ground-water region & Alluvial Mountain Valleys & $\mathrm{H} 1$ & \\
\hline $12 \mathrm{C}$ & Hawaiian islands Ground-water region & Volcanic Uplands & $\mathrm{H} 5$ & \\
\hline $12 \mathrm{D}$ & Hawaiian islands Ground-water region & Coastal Beaches & $\mathrm{H} 1$ & \\
\hline $13 \mathrm{~A}$ & Alaska Ground-Water Project & Alluvium & $\mathrm{H} 1$ & \\
\hline $13 \mathrm{~B}$ & Alaska Ground-Water Project & $\begin{array}{c}\text { Glacial and Glaciolacustrine Deposits of the } \\
\text { Interior Valleys }\end{array}$ & $\mathrm{H} 1$ & \\
\hline $13 \mathrm{C}$ & Alaska Ground-Water Project & Coastal-Lowland Deposits & $\mathrm{H} 1$ & \\
\hline 13D & Alaska Ground-Water Project & Bedrock of the Uplands and Mountains & $\mathrm{H} 3$ & \\
\hline
\end{tabular}

Note: ${ }^{* *}$ For finding the scenarios corresponding to given Levels, refer USER'S MANUAL "Chlorinated Solvent Plume" Scenarios. 


\section{SCENARIO NUMBER 1}

\section{Simple Fast Flow and}

\section{Anaerobic}
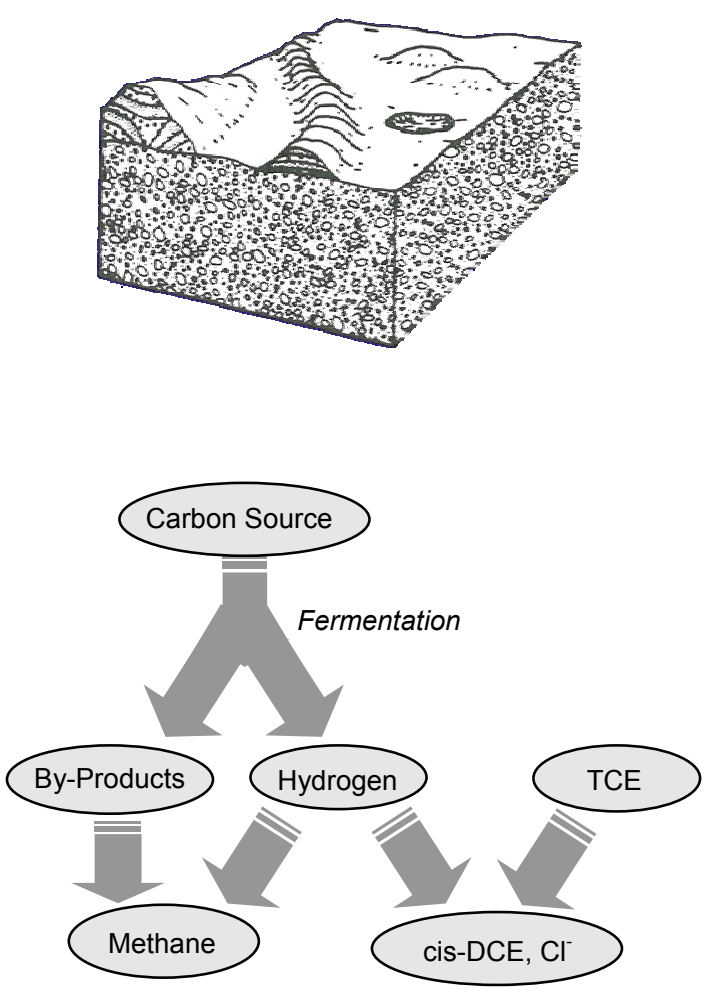


\begin{tabular}{|l|l|l|}
\hline Aerobic & Anoxic & Anaerobic \\
\hline
\end{tabular}

\section{SCENARIO 1 DESCRIPTION: SIMPLE FAST FLOW and ANAEROBIC}

The hydrogeologic setting and geochemical setting below define the basic scenario type. This scenario has the following characteristics:

\section{Hydrogeologic Setting}

"Simple and Fast" Hydrogeology:

- Only one hydrogeologic unit

- Relatively uniform hydraulic conductivity

- Relatively high groundwater seepage velocity

(see Section 2.1 and Appendix 1 for more information)

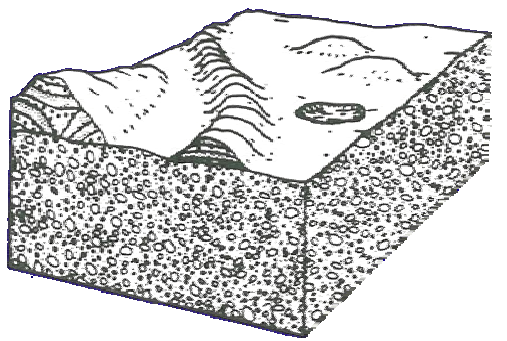

Block Diagram of Outwash Aquifer from DRASTIC System

\section{Geochemical Setting}

"Anaerobic" Geochemistry:

- Dissolved oxygen and redox are low

- Low to moderate concentrations of competing electron acceptors (nitrate, sulfate)

- Methane being produced.

(see Section 2.2 for more information)

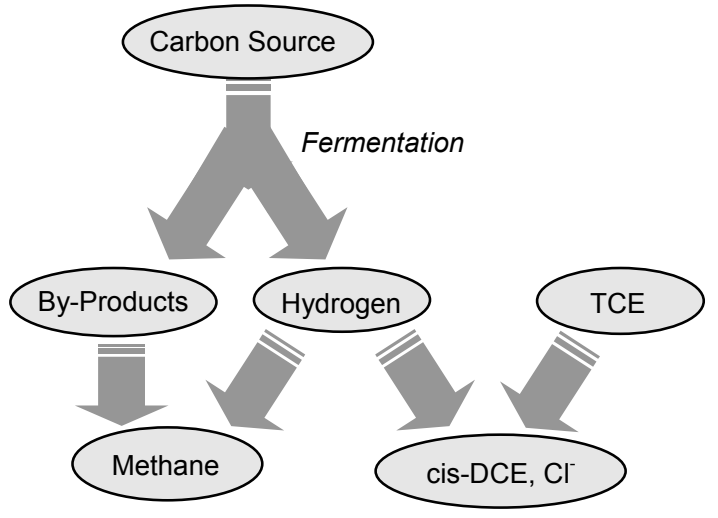

Example Reactions for "Anaerobic" Geochemical Setting 
WSRC-STI_2006-00096, Rev. 1

October 2, 2006

\section{KEY DECHLORINATION REACTIONS}

\section{Reaction Overview}

The chart to the right shows which reactions are likely to occur, which occur but at a slow rate, which may occur under specific conditions, and which are unlikely to occur.

\section{Typically Biodegradable Parent Compounds}

These compounds are typically degradable under anaerobic conditions:

- PCE

- TCE

- 1,1,1-TCA

- $1,2-D C A$

- $\quad \mathrm{CT}$

\section{Typical Daughter Products}

Daughter products that may be present depending on the parent compound and the reactions listed to the right:

- TCE

- $\quad$ cis 1,2-DCE

- VC

- 1,2-DCA

- 1,1-DCE

- $\mathrm{CF}$

See Section 5.1 for more information about reactions

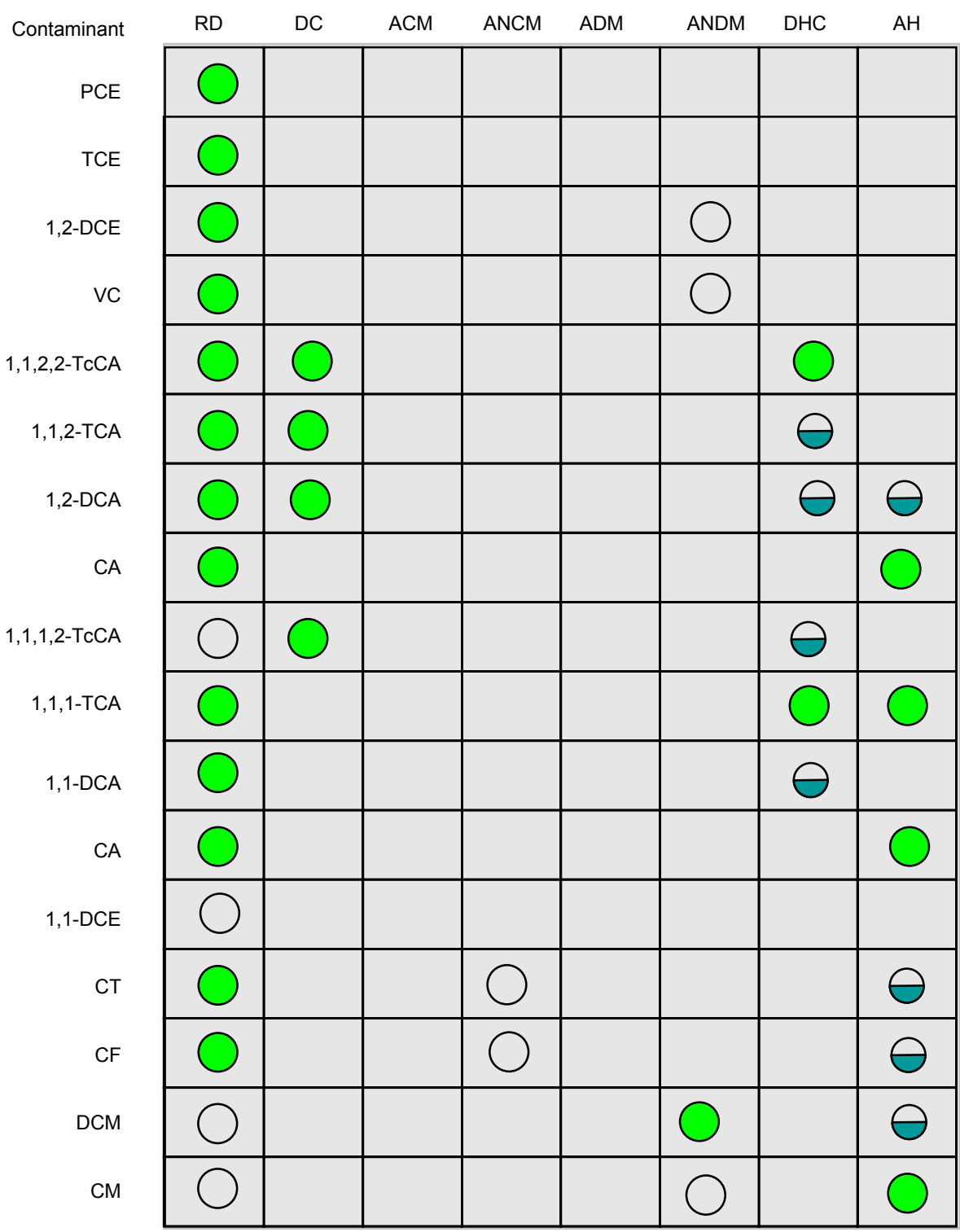

REACTIONS

\begin{tabular}{|c|l|}
\hline ACM & Aerobic Co-Metabolism \\
\hline ANCM & Anaerobic Co-Metabolism \\
\hline ADM & Aerobic Direct Metabolism \\
\hline ANDM & Anaerobic Direct Metabolism \\
\hline DHC & Dehydrochlorination (abiotic) \\
\hline AH & Abiotic Hydrolysis \\
\hline DC & Dichloroelimination (biotic) \\
\hline RD & Reductive Dechlorination (hydrogenolysis) \\
\hline
\end{tabular}




\section{EFFECT OF MODIFYING FACTORS}

\begin{tabular}{|c|c|}
\hline MODIFYING FACTOR & KEY POINTS \\
\hline Source Strength & $\begin{array}{l}\text { IF YOU HAVE A STRONG SOURCE: } \\
\text { - Plumes (both parent and daughter compounds) may be longer } \\
\text { - } \quad \text { Source zones may persist for longer periods of time } \\
\text { - More likely to need EA or source control measures } \\
\text { IF YOU HAVE A MODERATE SOURCE: } \\
\text { - Intermediate condition between Strong and Weak Source } \\
\text { IF YOU HAVE A WEAK SOURCE: } \\
\text { - Plumes may be shorter } \\
\text { - Source zones may not persist as long } \\
\text { - MNA alone or MNA with EA more likely to be sufficient }\end{array}$ \\
\hline Source & $\begin{array}{l}\text { IF YOU HAVE MOSTLY A VADOSE ZONE SOURCE: } \\
\text { - Source may appear small due to dilution but can be large } \\
\text { - } \quad \text { Sources in clay vadose zones will be weaker but more long-lived than } \\
\text { - } \quad \text { Pandy vadose zone } \\
\text { IF YOU HAVE A SUBMERGED SOURCE: } \\
\text { - Simple, fast hydrogeology means matrix diffusion will be less important } \\
\text { - } \quad \text { Source mass flux can decrease relatively rapidly as DNAPL fingers } \\
\text { - } \quad \text { dissolve } \\
\text { IF YOU HAVE A MIXED SOURCE: } \\
\text { - } \quad \text { May have combination of the factors above }\end{array}$ \\
\hline Travel Time to Receptors & $\begin{array}{l}\text { CLOSE RECEPTORS (<2 YEARS TRAVEL TIME) } \\
\text { - More intensive monitoring system likely to be needed due to potential } \\
\text { - } \\
\text { Morious consequences in event of failure of MNA/EA. } \\
\text { MODERATE RECEPTORS (>2 but < } 5 \text { YEARS TRAVEL TIME) } \\
\text { - Represents middle-ground case } \\
\text { DISTANT RECEPTORS (>5 YEARS TRAVEL TIME) } \\
\text { - Less intensive monitoring system likely to be needed due to lower } \\
\text { - } \\
\text { potential for serious consequences in event of failure of MNA/EA. }\end{array}$ \\
\hline Plume Stability & $\begin{array}{l}\text { EXPANDING OR PERTURBED }{ }^{1} \text { PLUME } \\
-\quad \text { More intensive monitoring system likely to be needed } \\
\text { STABLE PLUME } \\
\text { SHRINKING PLUME } \\
\text { - Less intensive monitoring system likely to be needed } \\
\text { - MNA alone likely to be sufficient } \\
{ }^{1} \text { For instance if the plume has been impacted by a previous remedy such as P\&T }\end{array}$ \\
\hline
\end{tabular}




\section{WILL MNA WORK?}

\section{Potential for MNA Processes to Control Plume}

This scenario often is well suited for natural attenuation processes to manage the contaminants in the plume or plume segment. The anaerobic conditions almost always mean that biodegradation processes are active.

In a fast-flowing heterogeneous aquifer where anaerobic conditions are present uniformly throughout the plume, relatively high rates of contaminant degradation may be needed to stabilize the plume. With the typical reductive dechlorination processes that are occurring under these conditions, it would be expected that the daughter product plume would be larger than the parent product plume because the degradation rate of daughters is typically slower than the rate for the parents.

At some Scenario 1 sites, "DCE stall" may be of concern. DCE stall is an informal term typically used to describe conditions at chlorinated ethene sites where the cis-1,2-DCE "stalls outs" or exhibits a very low conversion rate to VC. This DCE "stall" condition has been ascribed to a variety of factors, including:

- $\quad$ Lack of the necessary microbiological communities that are required to degrade cis-1,2-DCE to VC;

- The direct conversion of cis-1,2-DCE to carbon dioxide, which makes it appear that cis-1,2-DCE is not being biodegraded because VC is not being produced; but in fact the cis-1,2-DCE is being biodegraded by direct oxidation to carbon dioxide;

- Conditions which are anaerobic enough to support the conversion of TCE to cis-1,2-DCE but not anaerobic enough to support the conversion from cis-1,2-DCE to VC by reductive dechlorination;

- $\quad$ Toxicity effects caused at sites where sulfate reducers are producing hydrogen sulfide $\left(\mathrm{H}_{2} \mathrm{~S}\right)$, but the $\mathrm{H}_{2} \mathrm{~S}$ is not being precipitated fast enough by ferrous iron (a by-product of ferric iron reduction) to prevent toxicity effects in the cis-1,2-DCE degraders.

While the cause of cis-1,2-DCE stall is still being evaluated by a number of researchers, the main implication is that at some chlorinated ethene sites, cis-1,2-DCE plumes are expanding and not being controlled. DCE "stall" does not affect long-term sustainability of a reaction, but does determine if natural attenuation processes are sufficient to prevent migration of the plume.

\section{Key Sustainability Concept}

Fast-flowing plume segments can have high mass flux of contaminants leaving the source, and therefore high rates of degradation are often needed to attenuate the plume. Sufficient natural substrate (e.g., organic matter) or cocontaminants that serve as electron donors and can act as a substrate are needed to sustain high rates of degradation. The BIOBALANCE software system ${ }^{1}$ has a module designed to evaluate sustainability issues for anaerobic MNA reactions. Key input data are: i) mass fraction of solvents vs. donors in NAPL; OR ii) dissolvedphase concentrations of solvents and donors in the source zone.

${ }^{1}$ Developed by Groundwater Services (www.gsi-net.com), with the support of the Savannah River National Laboratory and $\mathrm{DOE}$, to evaluate monitored natural attenuation at chlorinated solvent sites. 


\begin{tabular}{|c|c|}
\hline $\begin{array}{c}\text { Heterogeneous } \\
\text { Slow }\end{array}$ & $\begin{array}{c}\text { Fractured/ } \\
\text { Porous Rock }\end{array}$ \\
\hline
\end{tabular}

\begin{tabular}{|l|l|l|}
\hline Aerobic & Anoxic & Anaerobic \\
\hline
\end{tabular}

\section{HOW DO I CHARACTERIZE THIS TYPE OF SITE?}

\section{Actions Needed to Determine MNA Viability}

The following are key considerations for evaluating the viability of MNA at a Simple Fast Flow and Anaerobic type site:

- both parent compound and daughter compounds need to be delineated (the anaerobic setting means that a number of daughter products will likely be generated);

- confirm that anaerobic conditions are present throughout the entire plume segment;

- determine if plume(s) are expanding/perturbed, stable, or shrinking;

- determine the location of any receptors (if present) and determine the travel time from the edge of the plume to these receptors;

- a mass-balance type evaluation of the source zone will help to determine if the electron donor supply is sustainable over the long term.

\section{Key Monitoring Concepts}

You will likely need a simple set of transect wells along the plume centerline and some sentry wells - looks like the "text book" case due to the simple plume shape in a homogenous aquifer.

The fast hydrogeologic setting may mean the plume can be relatively large, and therefore require more monitoring points. In addition, the plume can achieve steady-state conditions (if it is going to) more quickly than for a slow hydrogeologic setting, so an extremely long temporal record (i.e., the number of years of monitoring data you have) may not be needed to determine plume stability.

\section{Key Uncertainty Concepts}

A key uncertainty may be the sustainability of MNA due to the high mass flux of the source that may be present (see key sustainability concept section). There is less uncertainty about the plume conditions in general under this scenario because the plume is likely to be fully developed and it will be evident if MNA is currently working. 


\section{HOW DO I ANALYZE DATA?}

In a fast-flowing aquifer, a plume is more likely to show concentration differences over a longer distance and the plume will become stable (if it is going to) in a shorter period of time than in slower-flow aquifers. Thus, a good first step in this type of aquifer is to examine plume maps, concentration vs. time at each well, and concentration vs. distance plots to determine whether the plume is attenuating. Concentrations should show a progression of parent to daughter products with distance. In most cases, this type of data will be sufficient for a fast-flowing aquifer where anaerobic conditions are present throughout the plume. A simple transport model such as BIOCHLOR can be helpful in analyzing and visualizing the data and expected plume conditions for comparison to field data. The chart below summarizes an approach for analyzing data at sites depending on whether the concentration data indicates that the plume is decreasing, stable, or increasing and the source type. As noted in the table, as the source gets stronger and the plume is less likely to be decreasing in extent, more information is needed to support selection of an MNA remedy.

\begin{tabular}{|c|c|c|c|}
\hline & \multicolumn{3}{|c|}{ PLUME STATUS } \\
\hline $\begin{array}{l}\text { CONTAMINANT } \\
\text { CONCENTRATIONS/GEOCHEMICAL } \\
\text { STATUS }\end{array}$ & $\begin{array}{l}\text { DECREASING OR } \\
\text { PROBABLY } \\
\text { DECREASING }\end{array}$ & STABLE & $\begin{array}{l}\text { INCREASING, } \\
\text { PROBABLY } \\
\text { INCREASING, OR } \\
\text { PERTURBED }^{1}\end{array}$ \\
\hline Weak Source & - Mass loss & $\begin{array}{l}\text { - Mass loss } \\
\text { - Geochemical } \\
\text { footprints }\end{array}$ & $\begin{array}{l}\text { - Mass loss } \\
\text { - Geochemical } \\
\text { footprints } \\
\text { - Simple Model }\end{array}$ \\
\hline Strong Source & $\begin{array}{l}\text { - Mass loss } \\
\text { - Geochemical } \\
\text { footprints }\end{array}$ & $\begin{array}{ll}\text { - } & \text { Mass loss } \\
\text { - } & \text { Geochemical } \\
\text { footprints } \\
\text { - Simple Model }\end{array}$ & $\begin{array}{l}\text { - Mass loss } \\
\text { - Geochemical } \\
\text { footprints } \\
\text { - Comprehensive } \\
\text { Model/ Special } \\
\text { Studies }\end{array}$ \\
\hline
\end{tabular}

${ }^{1}$ For instance if the plume has been impacted by a previous remedy such as P\&T.

To demonstrate mass loss construct these graphics:

- Concentration vs. time plots at individual wells;

- Concentration vs. distance plots, with multiple lines for different sampling events through time;

- Plume maps showing plume extent at different times (i.e., either panel maps, or one map with several plume boundaries for different times).

To show geochemical footprints make tables or figures that show:

- Daughter product production;

- Ethene/Ethane production;

- Chloride production (this may not work at many sites, however, due to background chloride);

- Low dissolved oxygen (shows geochemical conditions are ok);

- Methane and iron(II) distribution (indicators of anaerobic activity);

- Nitrate and sulfate distribution (indicators of competing electron acceptors);

To perform modeling, typical tools include the following:

- Simple transport model (analytical model, e.g., BIOCHLOR, BIOBALANCE ${ }^{1}$ );

- Comprehensive transport model (numerical model, e.g., RT3D).

If a special study is needed, some of the following may be applicable:

- Carbon/chlorine isotope analysis (indicator of degradation processes)

- Molecular probes (indicators of microbial activity)

- Microcosm tests (determine the reaction processes occurring at the site).

1 Developed by Groundwater Services (www.gsi-net.com), with the support of the Savannah River National Laboratory and $\mathrm{DOE}$, to evaluate monitored natural attenuation at chlorinated solvent sites. 


\begin{tabular}{|l|l|l|}
\hline Aerobic & Anoxic & Anaerobic \\
\hline
\end{tabular}

\section{WHAT ABOUT COSTS AND ENHANCEMENTS?}

Costs for evaluating and implementing MNA for this scenario are primarily dependent on the following items.

- $\quad$ Source strength - Stronger and longer lasting sources will be more costly especially due to the high groundwater flow rate.

- $\quad$ Depth to the plume and size of the plume - Deeper, larger plumes require more and costlier monitoring wells.

- $\quad$ Travel time to the receptor - Plumes closer to receptors will require more frequent monitoring especially with the high groundwater flow rate.

- $\quad$ Plume stability - Less stable plumes require more rigorous evaluation and monitoring to select and implement MNA especially with the high groundwater flow rate.

MNA may be a viable single remedy for the site. If it is determined that MNA may not meet remediation goals, a good first option is to evaluate the potential use of sustainable enhancements (enhanced attenuation). The objective being to adjust the attenuation conditions sufficiently such that the plume is controlled. If the enhancements are insufficient to control the plume, source control treatment may be required. In general, enhanced attenuation is less likely a viable option for a fast flow regime with a strong source, unless source treatment is undertaken initially. The sections below discuss options for enhanced attenuation and source control related to this scenario.

\section{Key Enhanced Attenuation Concepts}

Enhancements are presented organized by the different zones in which they may be applied: source zone (reduction of contaminant mass flux to plume); plume (enhanced attenuation processes); or discharge zone (enhanced attenuation processes). Within the source zone, enhancements can be applied as a hydraulic manipulation or as a passive source reduction (active source control is discussed in the next section). Within the plume and discharge zone, either biological (microbial or plant based) or abiotic (abiotic degradation, reactive barriers, sorption) attenuation processes can be enhanced. A description of potential enhanced attenuation approaches, and their applicability to Scenario 1 sites, is shown below. More detailed information about each technology listed below is available in Early et al., (2005). 


\begin{tabular}{|l|l|l|}
\hline Aerobic & Anoxic & Anaerobic \\
\hline
\end{tabular}

\section{Enhancements Summary Table}

\begin{tabular}{l}
\multicolumn{1}{c}{ ENHANCEMENT } \\
\hline SOURCE ZONE ENHA \\
Surface water or \\
groundwater \\
interception/diversion
\end{tabular}

Physical containment

Passive extraction

Enhanced

biodegradation

\section{DESCRIPTION}

Use of interception trenches or wells, surface covers, or phyto-covers (plants) to reduce water flux through source area and/or divert unwanted electron acceptors.

Use of grout walls and other physical containment

\section{Use of passive soil vapor extraction}

Injection of electron donor (e.g., HRC, molasses, veg oil) to enhance microbial degradation of the source.

$$
\text { Permeable reactive }
$$
barrier
Use of zero valent iron, reduced sediment iron, enhanced partitioning, or biological barrier to attenuate some of the contaminant flux at the downgradient edge of the source area.

\section{APPLICABILITY TO SCENARIO 1 SITES}

May be more difficult in high flow rate conditions. Likely more applicable using surface covers or phyto-covers if the source is primarily within the vadose zone.

Potentially applicable depending on the geometry of the source zone.

Useful if the source is primarily within the vadose zone.

Well suited for anaerobic sites; enhances existing biodegradation reactions. Suitable for most Scenario 1 sites. Simple hydrogeology makes application easier. Faster groundwater flow may require larger amounts of electron donor than in slower groundwater if electron acceptors are carried into the treatment zone.

May be more difficult and expensive to construct due to fast flow regime. Faster groundwater flows potentially result in more contaminant and competing electron acceptors passing through the barrier, requiring greater thickness to achieve desired treatment levels. Anaerobic conditions are helpful because oxygen concentration is low and will not disrupt barriers using anaerobic reactions.

\section{PLUME AND DISCHARGE ZONE ENHANCEMENTS}

Enhanced
biodegradation

Permeable reactive barrier
Injection of electron donor (e.g., HRC, molasses, veg oil) to enhance microbial degradation of the source.

Use of zero valent iron, reduced sediment iron, enhanced partitioning, or biological barrier to attenuate some of the contaminant flux within the plume.

Phytoextraction

Plant-based hydraulic control (plume enhancement only)

Use of plants to extract surface groundwater

Use of plants to control hydraulic gradient and slow groundwater
Well suited for anaerobic sites; enhances existing biodegradation reactions. Suitable for most Scenario 1 sites. Simple hydrogeology makes application easier. Faster groundwater flow may require larger amounts of electron donor than in slower groundwater if electron acceptors are carried into the treatment zone.

May be more difficult and expensive to construct due to fast flow regime. Faster groundwater flows potentially result in more contaminant and competing electron acceptors passing through the barrier, requiring greater thickness to achieve desired treatment levels. Anaerobic conditions are helpful because oxygen concentration is low and will not disrupt barriers using anaerobic reactions.

May be less effective for fast groundwater flow conditions.
May be less effective for fast groundwater flow conditions. 


\begin{tabular}{|c|c|c|c|c|}
\hline $\begin{array}{c}\text { Simple } \\
\text { Fast }\end{array}$ & $\begin{array}{c}\text { Simple } \\
\text { Slow }\end{array}$ & $\begin{array}{c}\text { Heterogeneous } \\
\text { Fast }\end{array}$ & $\begin{array}{c}\text { Heterogeneous } \\
\text { Slow }\end{array}$ & $\begin{array}{c}\text { Fractured/ } \\
\text { Porous Rock }\end{array}$ \\
\hline
\end{tabular}

\begin{tabular}{|l|c|c|}
\hline \multicolumn{2}{|c|}{ GEOCHEMICAL SETTING } \\
\hline Aerobic & Anoxic & Anaerobic \\
\hline
\end{tabular}

\section{Key Source Control Concepts}

A description of potential source control measures, and their applicability to Scenario 1 sites, is shown below. Note that source control measures are unlikely to achieve complete restoration at a site, and some source material is always left behind after treatment (U.S. EPA, 2003; McGuire et al., 2006).

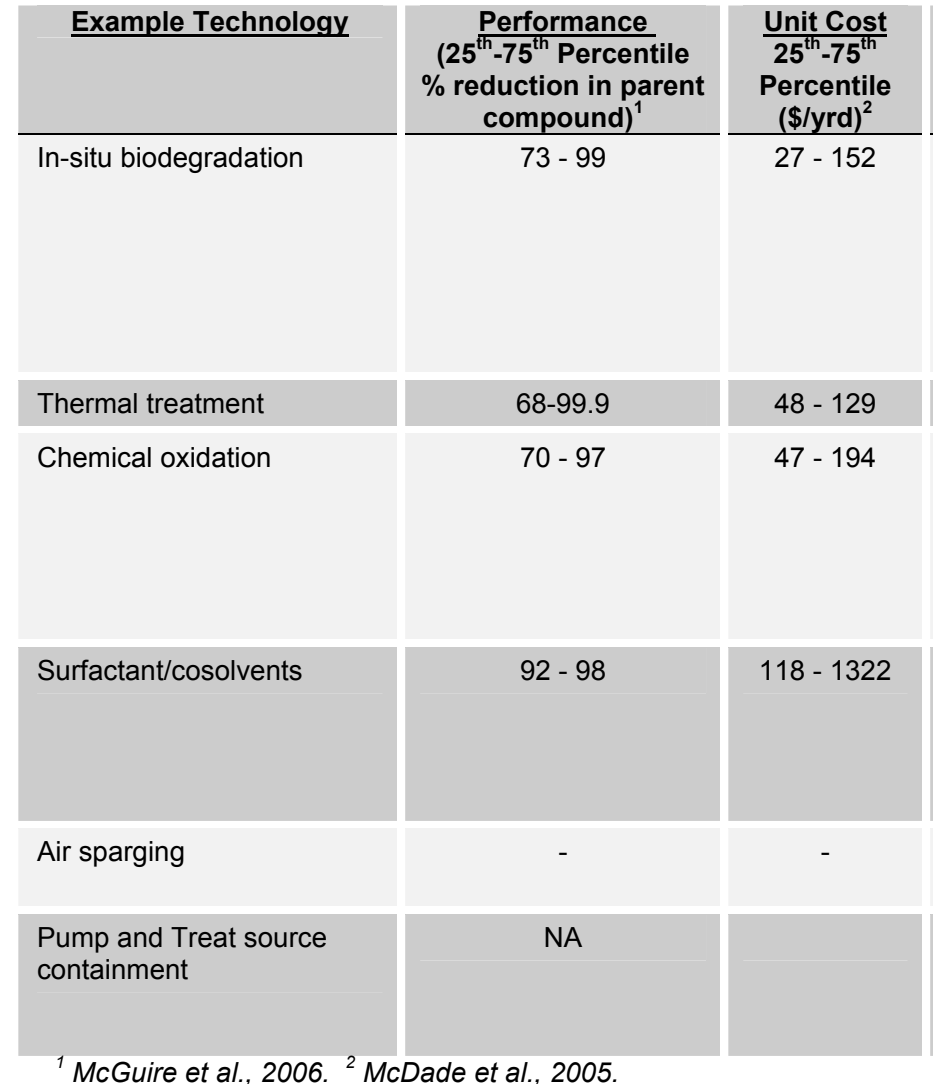

\section{Applicability to Scenario 1 Sites}

Well suited for anaerobic sites; enhances existing biodegradation reactions. Suitable for most Scenario 1 sites. Simple hydrogeology makes application easier. Faster groundwater flow may require larger amounts of electron donor than in slower groundwater if electron acceptors are carried into the treatment zone. Least expensive treatment option.

Does not appear to disrupt MNA after treatment.

Removes more total CVOCs than enhanced biodegradation but shows more rebound ${ }^{1}$. Can change geochemistry of Scenario 1 site to aerobic conditions for some period after treatment. Can change microbial population and composition. Simple hydrogeology makes application easier. May be more suitable for anoxic or aerobic sites.

High treatment efficiency but much higher cost. Costs reflect some expensive pilot-scale projects. Simple hydrogeology makes application easier. Some surfactants/cosolvents can serve as electron donors for subsequent anaerobic biodegradation reactions.

Not recommended at most sites. Addition of oxygen can disrupt anaerobic processes.

Due to the high groundwater flow rate, a large system may be required. This approach does not reduce mass significantly compared to the rate of mass loss without P\&T and may need to be operated for a long time. 


\section{SCENARIO NUMBER 2}

\section{Simple Fast Flow} and Anoxic
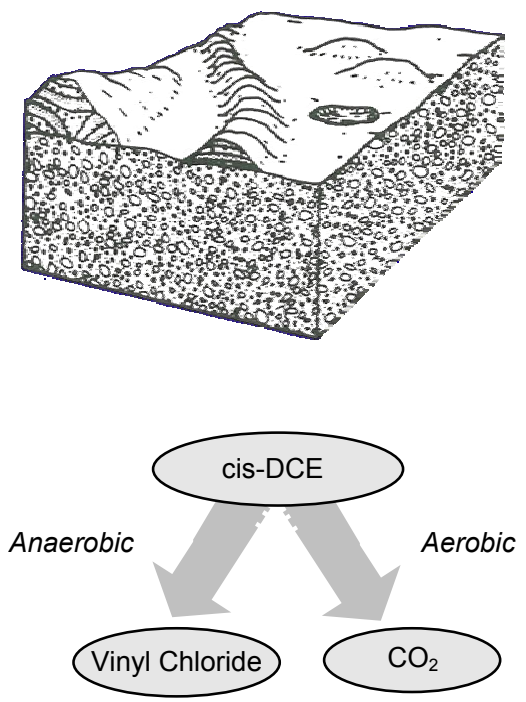


\begin{tabular}{|l|l|l|}
\hline Aerobic & Anoxic & Anaerobic \\
\hline
\end{tabular}

\section{SCENARIO 2 DESCRIPTION: SIMPLE FAST FLOW and ANOXIC}

The hydrogeologic setting and geochemical setting below define the basic scenario type. This scenario has the following characteristics:

\section{Hydrogeologic Setting}

"Simple and Fast" Hydrogeology:

- Only one hydrogeologic unit

- Relatively uniform hydraulic conductivity

- Relatively high groundwater seepage velocity

(see Section 2.1 and Appendix 1 for more information)

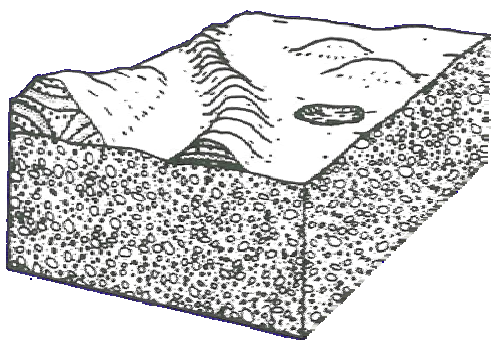

Block Diagram of Outwash Aquifer from DRASTIC System

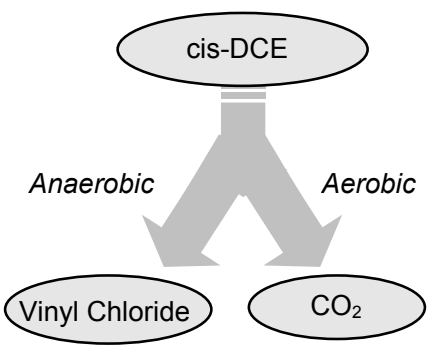

Example Reactions for "Anoxic" Geochemical Setting 
WSRC-STI-2006-00096, Rev. 1

Occtober 2, 2006

HYDROGEOLOGIC SETTING

GEOCHEMICAL SETTING

Simple

Fast

Simple

Heterogeneous

Heterogeneous

Fractured/

\begin{tabular}{l|l|l|l} 
Slow & Fast & Slow & Porous Rock \\
\hline
\end{tabular}

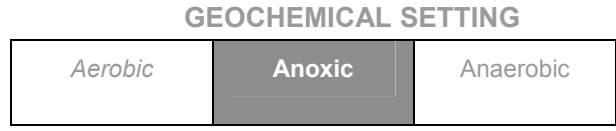

\section{KEY DECHLORINATION REACTIONS}

\section{Reaction Overview}

The chart to the right shows which reactions are likely to occur, which occur but at a slow rate, which may occur under specific conditions, and which are unlikely to occur.

\section{Typically Biodegradable Parent Compounds}

These compounds may be degradable under anoxic conditions:

- PCE

- TCE

- 1,1,1-TCA

- 1,2-DCA

- $\mathrm{CT}$

\section{Typical Daughter Products}

Daughter products that may be present depending on the parent compound and the reactions listed to the right:

- TCE

- $\quad$ cis 1,2-DCE

- VC

- 1,2-DCA

- 1,1-DCE

- $\mathrm{CF}$

See Section 5.1 for more information a bout reactions

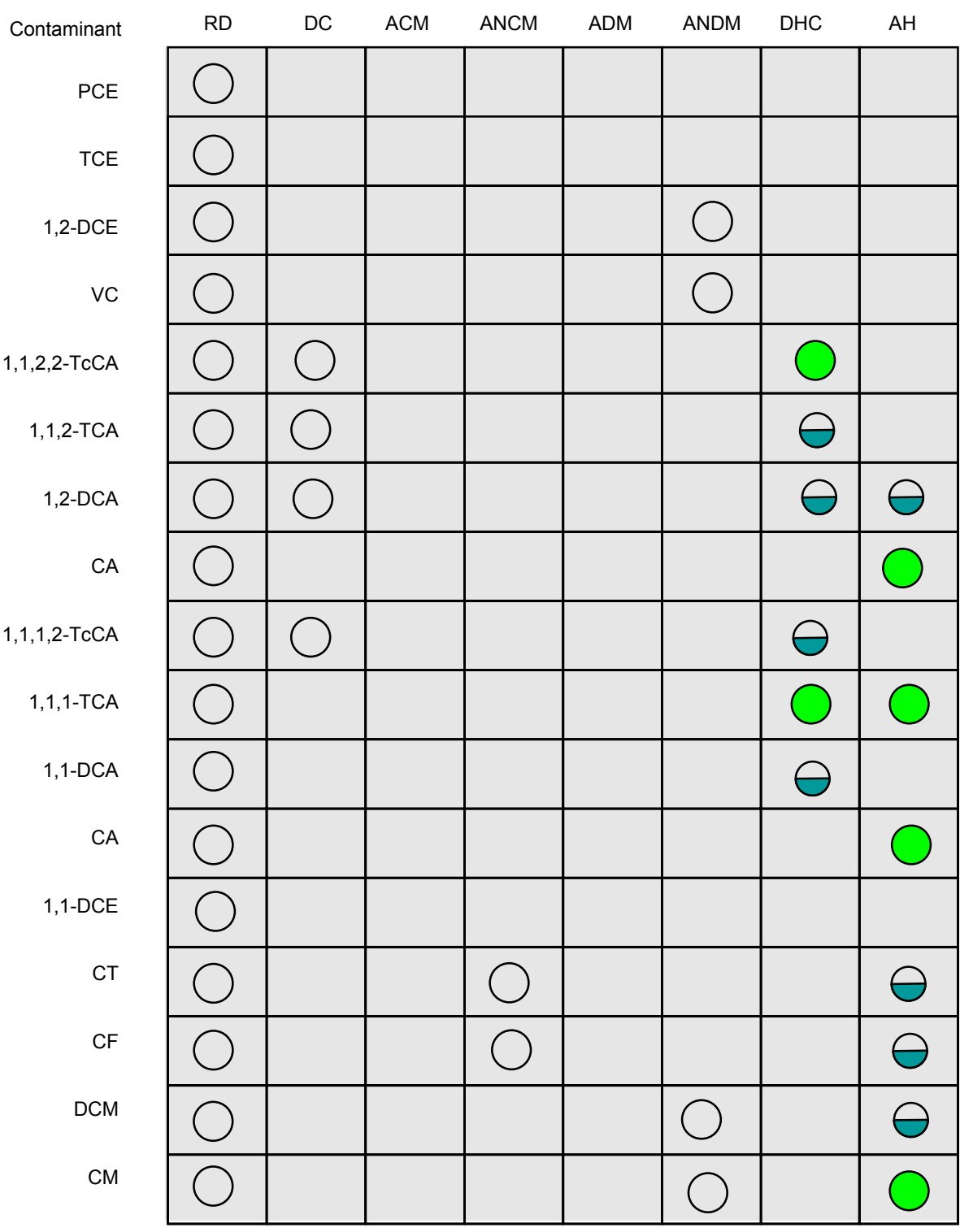

REACTIONS

\begin{tabular}{|l|}
\hline Key: \\
\hline Highly Likely to occur \\
Highly likely to occur, but a slow rate \\
May occur under specific conditions \\
Highly Unlikely to occur
\end{tabular}

\begin{tabular}{|c|l|}
\multicolumn{1}{|c|}{ REACTIONS } \\
\hline ACM & Aerobic Co-Metabolism \\
\hline ANCM & Anaerobic Co-Metabolism \\
\hline ADM & Aerobic Direct Metabolism \\
\hline ANDM & Anaerobic Direct Metabolism \\
\hline DHC & Dehydrochlorination (abiotic) \\
\hline AH & Abiotic Hydrolysis \\
\hline DC & Dichloroelimination (biotic) \\
\hline RD & Reductive Dechlorination (hydrogenolysis) \\
\hline
\end{tabular}




\section{EFFECT OF MODIFYING FACTORS}

\begin{tabular}{|c|c|}
\hline MODIFYING FACTOR & KEY POINTS \\
\hline Source Strength & $\begin{array}{l}\text { IF YOU HAVE A STRONG SOURCE: } \\
\text { - } \quad \text { Plumes (both parent and daughter compounds) may be longer } \\
\text { - } \quad \text { Source zones may persist for longer periods of time } \\
\text { - } \quad \text { More likely to need EA or source control measures } \\
\text { IF YOU HAVE A MODERATE SOURCE: } \\
\text { - Intermediate condition between Strong and Weak Source } \\
\text { IF YOU HAVE A WEAK SOURCE: } \\
\text { - } \quad \text { Plumes may be shorter } \\
\text { - } \quad \text { Source zones may not persist as long } \\
\text { - } \quad \text { MNA alone or MNA with EA more likely to be sufficient }\end{array}$ \\
\hline Source & $\begin{array}{l}\text { IF YOU HAVE MOSTLY A VADOSE ZONE SOURCE: } \\
\text { - Source may appear small due to dilution but can be large } \\
\text { - } \quad \text { Sources in clay vadose zones will be weaker but more long-lived than } \\
\text { sandy vadose zone } \\
\text { - } \quad \text { Plumes will be thinner and closer to water table } \\
\text { IF YOU HAVE A SUBMERGED SOURCE: } \\
\text { - Simple, fast hydrogeology means matrix diffusion will be less important } \\
\text { - } \quad \text { Source mass flux can decrease relatively rapidly as DNAPL fingers } \\
\text { - } \quad \text { dissolve } \\
\text { IF YOU HAVE A MIXED SOURCE: } \\
\text { - } \quad \text { May have combination of the factors above }\end{array}$ \\
\hline Travel Time to & $\begin{array}{l}\text { CLOSE RECEPTORS (<2 YEARS TRAVEL TIME) } \\
\text { - More intensive monitoring system likely to be needed due to potential } \\
\text { serious consequences in event of failure of MNA/EA. } \\
\text { MODERATE RECEPTORS (>2 but < } 5 \text { YEARS TRAVEL TIME) } \\
\text { - Represents middle-ground case } \\
\text { DISTANT RECEPTORS (>5 YEARS TRAVEL TIME) } \\
\text { - Less intensive monitoring system likely to be needed due to lower } \\
\text { - } \quad \text { Motential for serious consequences in event of failure of MNA/EA. }\end{array}$ \\
\hline Plume Stability & $\begin{array}{l}\text { EXPANDING OR PERTURBED }{ }^{1} \text { PLUME } \\
\text { - More intensive monitoring system likely to be needed } \\
\text { STABLE PLUME } \\
\text { SHRINKING PLUME } \\
\text { - } \quad \text { Represents middle-ground case } \\
\text { - MNA alone likely to be sufficient } \\
{ }^{1} \text { For instance if the plume has been impacted by a previous remedy such as P\&T. }\end{array}$ \\
\hline
\end{tabular}




\begin{tabular}{|c|c|c|c|c|}
\hline $\begin{array}{c}\text { Simple } \\
\text { Fast }\end{array}$ & $\begin{array}{c}\text { Simple } \\
\text { Slow }\end{array}$ & $\begin{array}{c}\text { Heterogeneous } \\
\text { Fast }\end{array}$ & $\begin{array}{c}\text { Heterogeneous } \\
\text { Slow }\end{array}$ & $\begin{array}{c}\text { Fractured/ } \\
\text { Porous Rock }\end{array}$ \\
\hline
\end{tabular}

\begin{tabular}{|l|l|l|}
\hline Aerobic & Anoxic & Anaerobic \\
\hline
\end{tabular}

\section{WILL MNA WORK?}

\section{Potential for MNA Processes to Control Plume}

Because at Scenario 2 sites there are not clear indicators that the type of conditions conducive to MNA are present, it is initially uncertain whether natural attenuation processes will be suitable to manage the contaminants in the plume or plume segment. Typically, more in-depth investigation of the site attenuation processes and more rigorous monitoring are needed to evaluate the extent of natural attenuation processes and the ability of MNA to meet the remediation objectives. Some form of enhanced attenuation may be needed to couple with MNA as the remedy.

In a fast-flowing heterogeneous aquifer, relatively high rates of contaminant degradation may be needed to stabilize the plume. If reductive dechlorination processes are occurring under these conditions, it would be expected that the daughter product plume would be larger than the parent product plume because the degradation rate of daughters is typically slower than the rate for the parents.

If the plume is shown to be either stable or shrinking then natural attenuation processes (primarily reductive dechlorination) alone have been vigorous enough to date to prevent further migration of the plume or plume segment. Under these conditions MNA may be appropriate, but it may still be difficult to identify the specific attenuation mechanism under the anoxic geochemical conditions.

At some Scenario 2 sites, "DCE stall" may be of concern and an indication that conditions are not suitable for complete dechlorination of the source contaminants. DCE stall is an informal term typically used to describe conditions at chlorinated ethene sites where the cis-1,2-DCE "stalls outs" or exhibits a very low conversion rate to VC. This DCE "stall" condition has been ascribed to a variety of factors, including:

- $\quad$ Lack of the necessary microbiological communities that are required to degrade cis-1,2-DCE to VC;

- The direct conversion of cis-1,2-DCE to carbon dioxide, which makes it appear that cis-1,2-DCE is not being biodegraded because VC is not being produced; but in fact the cis-1,2-DCE is being biodegraded by direct oxidation to carbon dioxide;

- Conditions which are anaerobic enough to support the conversion of TCE to cis-1,2-DCE but not anaerobic enough to support the conversion from cis-1,2-DCE to VC by reductive dechlorination;

- Toxicity effects caused at sites where sulfate reducers are producing hydrogen sulfide $\left(\mathrm{H}_{2} \mathrm{~S}\right)$, but the $\mathrm{H}_{2} \mathrm{~S}$ is not being precipitated fast enough by ferrous iron (a by-product of ferric iron reduction) to prevent toxicity effects in the cis-1,2-DCE degraders.

While the cause of cis-1,2-DCE stall is still being evaluated by a number of researchers, the main implication is that at some chlorinated ethene sites, cis-1,2-DCE plumes are expanding and not being controlled. DCE "stall" does not affect long-term sustainability of a reaction, but does determine if natural attenuation processes are sufficient to prevent migration of the plume.

\section{Key Sustainability Concept}

Fast-flowing plume segments can have high mass flux of contaminants leaving the source, and therefore high rates of degradation are often needed to attenuate the plume. Sufficient natural substrate (e.g., organic matter) or cocontaminants that serve as electron donors and can act as a substrate are needed to sustain high rates of degradation for the anaerobic reactions using the chlorinated solvent as an electron acceptor. For some contaminants under anoxic conditions, biological reactions use the chlorinated solvent as the electron donor. At Scenario 2 sites, non-biologically catalyzed attenuation processes may be the primary attenuation processes. In this case, the processes are likely sustainable, but may be difficult to identify and quantify. 


\section{HOW DO I CHARACTERIZE THIS TYPE OF SITE?}

\section{Actions Needed to Determine MNA Viability}

The following are key considerations for evaluating the viability of MNA at a Simple Fast Flow and Anoxic type site:

- assess the site geochemical, hydraulic, and contaminant conditions in detail to assess the type and extent/rate of attenuation processes - this assessment may require significant effort depending on the site conditions, however, the uniform hydraulic conditions will help simplify some parts of the assessment;

- both parent compound and daughter compounds need to be delineated (the anoxic setting means that a number of daughter products may be generated);

- determine if plume(s) are expanding/perturbed, stable, or shrinking;

- determine the location of any receptors (if present) and determine the travel time from the edge of the plume to these receptors;

- a mass-balance type evaluation of the source zone will help to determine if the electron donor supply is sustainable over the long term if the attenuation reactions are determined to be primarily anaerobic dechlorination with the contaminant acting as the electron acceptor.

\section{Key Monitoring Concepts}

You will likely need a set of transect wells along the plume centerline and some sentry wells - looks like the "text book" case due to the simple plume shape in a homogenous aquifer. However, if characterization indicates that there is spatial variability in the geochemical conditions, monitoring for the specific geochemical areas may increase the number of wells needed compared to sites with more uniform geochemical conditions.

The fast hydrogeologic setting may mean the plume can be relatively large, and therefore require more monitoring points. In addition, the plume can achieve steady-state conditions (if it is going to) more quickly than for a slow hydrogeologic setting, so an extremely long temporal record (i.e., the number of years of monitoring data you have) may not be needed to determine plume stability.

\section{Key Uncertainty Concepts}

A key uncertainty may be the sustainability of MNA due to the high mass flux of the source that may be present (see key sustainability concept section). There may be less uncertainty about the plume conditions in general under this scenario if the plume appears to be fully developed and it is evident from a short duration of contaminant monitoring whether MNA is currently working. However, the anoxic geochemical setting may cause considerable uncertainty in evaluating MNA because it may be difficult to identify and quantify the attenuation processes. 


\begin{tabular}{|c|c|c|c|c|}
\hline $\begin{array}{c}\text { Simple } \\
\text { Fast }\end{array}$ & $\begin{array}{c}\text { Simple } \\
\text { Slow }\end{array}$ & $\begin{array}{c}\text { Heterogeneous } \\
\text { Fast }\end{array}$ & $\begin{array}{c}\text { Heterogeneous } \\
\text { Slow }\end{array}$ & $\begin{array}{c}\text { Fractured/ } \\
\text { Porous Rock }\end{array}$ \\
\hline
\end{tabular}

\begin{tabular}{|l|c|c|}
\hline \multicolumn{2}{|c|}{ GEOCHEMICAL SETTING } \\
\hline Aerobic & Anoxic & Anaerobic \\
\hline
\end{tabular}

\section{HOW DO I ANALYZE DATA?}

In a fast-flowing aquifer, a plume is more likely to show concentration differences over a longer distance and the plume will become stable (if it is going to) in a shorter period of time than in slower-flow aquifers. A good first step in this type of aquifer is to examine plume maps, concentration vs. time at each well, and concentration vs. distance plots to determine whether the plume is attenuating. Concentrations may not show a progression of parent to daughter products with distance. Thus, it is likely that contaminant monitoring over a period of time will be needed to establish trends in the plume size and concentration data. In some cases, this type of data will be sufficient for a fastflowing aquifer with anoxic conditions. A simple transport model such as BIOCHLOR can be helpful as a screening tool in analyzing and visualizing the data and expected plume conditions for comparison to field data. However, BIOCHLOR will not be sufficient to describe the range of attenuation processes that may be important under anoxic geochemical conditions. As such, more complex numerical modeling is more likely to be needed as part of MNA evaluation for Scenario 2 sites. To support this more complex analysis, microcosm tests, molecular probes, and more detailed field measurements may be required. The chart below summarizes an approach for analyzing data at sites depending on whether the concentration data indicates that the plume is decreasing, stable, or increasing and the source type. As noted in the table, as the source gets stronger and the plume is less likely to be decreasing in extent, more information is needed to support selection of an MNA remedy.

\begin{tabular}{|c|c|c|c|}
\hline & \multicolumn{3}{|c|}{ PLUME STATUS } \\
\hline $\begin{array}{l}\text { CONTAMINANT CONCENTRATIONS } \\
\text { /GEOCHEMICAL STATUS }\end{array}$ & $\begin{array}{c}\text { DECREASING OR } \\
\text { PROBABLY DECREASING }\end{array}$ & STABLE & $\begin{array}{c}\text { INCREASING, } \\
\text { PROBABLY INCREASING, } \\
\text { OR PERTURBED }^{1}\end{array}$ \\
\hline Weak Source & - Mass loss & $\begin{array}{ll}\text { - } & \text { Mass loss } \\
\text { - } & \text { Geochemical footprints } \\
\text { - } & \text { Simple Model/Special } \\
\text { Studies }\end{array}$ & $\begin{array}{l}\text { - } \text { Mass loss } \\
\text { - } \quad \text { Geochemical footprints } \\
\text { - Comprehensive Model/ } \\
\text { Special Studies }\end{array}$ \\
\hline Strong Source & $\begin{array}{ll}\text { - } & \text { Mass loss } \\
\text { - } & \text { Geochemical footprints } \\
\text { - } & \text { Simple Model }\end{array}$ & $\begin{array}{l}\text { - } \text { Mass loss } \\
\text { - } \quad \text { Geochemical footprints } \\
\text { - } \quad \text { Comprehensive Model/ } \\
\text { Special Studies }\end{array}$ & $\begin{array}{l}\text { - } \text { Mass loss } \\
\text { - } \quad \text { Geochemical footprints } \\
\text { - } \quad \text { Comprehensive Model/ } \\
\text { Special Studies }\end{array}$ \\
\hline
\end{tabular}

${ }^{1}$ For instance if the plume has been impacted by a previous remedy such as P\&T.

To demonstrate mass loss construct these graphics:

- Concentration vs. time plots at individual wells;

- Concentration vs. distance plots, with multiple lines for different sampling events through time;

- Plume maps showing plume extent at different times (i.e., either panel maps, or one map with several plume boundaries for different times).

To show geochemical footprints make tables or figures that show:

- Daughter product production;

- Ethene/Ethane production;

- Chloride production (this may not work at many sites, however, due to background chloride);

- Low dissolved oxygen (shows anoxic geochemical conditions);

- Methane and iron(II) distribution (indicators of anaerobic activity);

- Nitrate and sulfate distribution (indicators of competing electron acceptors);

To perform modeling, typical tools include the following:

- Simple transport model (analytical model, e.g., BIOCHLOR, BIOBALANCE ${ }^{1}$ );

- Comprehensive transport model (numerical model, e.g., RT3D).

If a special study is needed, some of the following may be applicable:

- Carbon/chlorine isotope analysis (indicator of degradation processes)

- Molecular probes (indicators of microbial activity when other geochemical indicators are ambiguous);

- Microcosm tests (determine the reaction processes occurring at the site).

${ }^{1}$ Developed by Groundwater Services (www.gsi-net.com), with the support of the Savannah River National Laboratory and $\mathrm{DOE}$, to evaluate monitored natural attenuation at chlorinated solvent sites. 


\begin{tabular}{|c|c|c|c|c|}
\hline $\begin{array}{c}\text { Simple } \\
\text { Fast }\end{array}$ & $\begin{array}{c}\text { Simple } \\
\text { Slow }\end{array}$ & $\begin{array}{c}\text { Heterogeneous } \\
\text { Fast }\end{array}$ & $\begin{array}{c}\text { Heterogeneous } \\
\text { Slow }\end{array}$ & $\begin{array}{c}\text { Fractured/ } \\
\text { Porous Rock }\end{array}$ \\
\hline
\end{tabular}

\begin{tabular}{|l|c|c|}
\hline \multicolumn{2}{|c|}{ GEOCHEMICAL SETTING } \\
\hline Aerobic & Anoxic & Anaerobic \\
\hline
\end{tabular}

\section{WHAT ABOUT COSTS AND ENHANCEMENTS?}

Costs for evaluating and implementing MNA for this scenario are primarily dependent on the following items.

- $\quad$ Source strength - Stronger and longer lasting sources will be more costly especially due to the high groundwater flow rate.

- $\quad$ Depth to the plume and size of the plume - Deeper, larger plumes require more and costlier monitoring wells.

- $\quad$ Travel time to the receptor - Plumes closer to receptors will require more frequent monitoring especially with the high groundwater flow rate.

- $\quad$ Plume stability - Less stable plumes require more rigorous evaluation and monitoring to select and implement MNA especially with the high groundwater flow rate.

- Extent of variability in geochemical conditions - More variability will likely require more characterization and monitoring to assess attenuation conditions within each different geochemical zone.

MNA may be a viable single remedy for the site. If it is determined that MNA may not meet remediation goals, a good first option is to evaluate the potential use of sustainable enhancements (enhanced attenuation). The objective being to adjust the attenuation conditions sufficiently such that the plume is controlled. If the enhancements are insufficient to control the plume, source control treatment may be required. In general, enhanced attenuation is less likely a viable option for a fast flow regime with a strong source, unless source treatment is undertaken initially. The sections below discuss options for enhanced attenuation and source control related to this scenario.

\section{Key Enhanced Attenuation Concepts}

Enhancements are presented organized by the different zones in which they may be applied: source zone (reduction of contaminant mass flux to plume); plume (enhanced attenuation processes); or discharge zone (enhanced attenuation processes). Within the source zone enhancements can be applied as a hydraulic manipulation or as a passive source reduction (active source control is discussed in the next section). Within the plume and discharge zone, either biological (microbial or plant based) or abiotic (abiotic degradation, reactive barriers, sorption) attenuation processes can be enhanced. A description of potential enhanced attenuation approaches, and their applicability to Scenario 2 sites, is shown below. More detailed information about each technology listed below is available in Early et al., (2005). 


\section{Enhancement Summary}

$\quad$ ENHANCEMENT
SOURCE ZONE ENHANCE
Surface water or
groundwater
interception/diversion

Physical containment

Passive extraction

Enhanced biodegradation

\section{DESCRIPTION}

Use of interception trenches or wells, surface covers, or phyto-covers (plants) to reduce water flux through source area and/or divert unwanted electron acceptors.

Use of grout walls and other physical containment

Use of passive soil vapor extraction

Injection of electron donor (e.g., HRC, molasses, vegetable oil) to enhance microbial degradation of the source. Permeable reactive
barrier
Use of zero valent iron, reduced sediment iron, enhanced partitioning, or biological barrier to attenuate some of the contaminant flux at the downgradient edge of the source area.

\section{PLUME AND DISCHARGE ZONE ENHANCEMENTS}

Enhanced

biodegradation

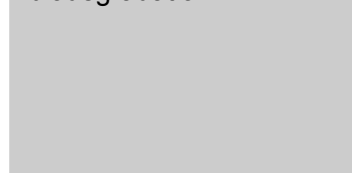

Permeable reactive barrier

\section{Phytoextraction}

Plant-based hydraulic control (plume enhancement only)
Injection of electron donor (e.g., HRC, molasses, vegetable oil) to enhance microbial degradation of the source.

Use of zero valent iron, reduced sediment iron, enhanced partitioning, or biological barrier to attenuate some of the contaminant flux within the plume.

Use of plants to extract contaminants from near surface groundwater

Use of plants to control gradient and slow groundwater
May be more difficult in high flow rate conditions. Likely more applicable using surface covers or phytocovers if the source is primarily within the vadose zone.

Potentially applicable depending on the geometry of the source zone.

Useful if the source is primarily within the vadose zone.

Potentially well suited for anoxic sites because oxygen concentrations are already low and anaerobic processes may be readily stimulated. Simple hydrogeology makes application easier. Faster groundwater flow may require larger amounts of electron donor than in slower groundwater if electron acceptors are carried into the treatment zone.

May be more difficult and expensive to construct due to fast flow regime. Faster groundwater flows potentially result in more contaminant and competing electron acceptors passing through the barrier, requiring greater thickness to achieve desired treatment levels. Anoxic conditions are helpful because oxygen concentration is low and will not disrupt barriers using anaerobic reactions.

Potentially well suited for anoxic sites because oxygen concentrations are already low and anaerobic processes may be readily stimulated. Simple hydrogeology makes application easier. Faster groundwater flow may require larger amounts of electron donor than in slower groundwater if electron acceptors are carried into the treatment zone.

May be more difficult and expensive to construct due to fast flow regime. Faster groundwater flows potentially result in more contaminant and competing electron acceptors passing through the barrier, requiring greater thickness to achieve desired treatment levels. Anoxic conditions are helpful because oxygen concentration is low and will not disrupt barriers using anaerobic reactions.

May be less effective for fast groundwater flow conditions.

May be less effective for fast groundwater flow conditions. 


\begin{tabular}{|c|c|c|c|c|}
\hline $\begin{array}{c}\text { Simple } \\
\text { Fast }\end{array}$ & $\begin{array}{c}\text { Simple } \\
\text { Slow }\end{array}$ & $\begin{array}{c}\text { Heterogeneous } \\
\text { Fast }\end{array}$ & $\begin{array}{c}\text { Heterogeneous } \\
\text { Slow }\end{array}$ & $\begin{array}{c}\text { Fractured/ } \\
\text { Porous Rock }\end{array}$ \\
\hline
\end{tabular}

\begin{tabular}{|l|c|c|}
\hline \multicolumn{2}{|c|}{ GEOCHEMICAL SETTING } \\
\hline Aerobic & Anoxic & Anaerobic \\
\hline
\end{tabular}

\section{Key Source Control Concepts}

A description of potential source control measures, and their applicability to Scenario 2 sites, is shown below. Note that source control measures are unlikely to achieve complete restoration at a site, and some source material is always left behind after treatment (U.S. EPA, 2003; McGuire et al., 2006).

\begin{tabular}{|c|c|c|c|}
\hline Example Technology & $\begin{array}{l}\text { Performance } \\
\text { Percentile }^{\text {th }}-75^{\text {th }} \\
\text { Percention in } \\
\text { parent } \\
\text { compound })^{1} \\
\end{array}$ & $\begin{array}{l}\frac{\text { Unit Cost }}{25^{\text {th }}-75^{\text {th }}} \\
\text { Percentile } \\
(\$ / y r d)^{2}\end{array}$ & Applicability to Scenario 2 Sites \\
\hline In-situ biodegradation & $73-99$ & $27-152$ & $\begin{array}{l}\text { Potentially well suited for anoxic sites because oxygen } \\
\text { concentrations are already low and anaerobic processes may } \\
\text { be readily stimulated. Simple hydrogeology makes application } \\
\text { easier. Faster groundwater flow may require larger amounts of } \\
\text { electron donor than in slower groundwater if electron acceptors } \\
\text { are carried into the treatment zone. Least expensive treatment } \\
\text { option. }\end{array}$ \\
\hline Thermal treatment & $68-99.9$ & $48-129$ & Does not appear to disrupt MNA after treatment. \\
\hline Chemical oxidation & $70-97$ & $47-194$ & $\begin{array}{l}\text { Removes more total CVOCs than enhanced biodegradation } \\
\text { but shows more rebound }{ }^{1} \text {. Can change geochemistry of } \\
\text { Scenario } 2 \text { site to aerobic conditions for some period after } \\
\text { treatment. Can change microbial population and composition. } \\
\text { Simple hydrogeology makes application easier. }\end{array}$ \\
\hline Surfactant/cosolvents & $92-98$ & $118-1322$ & $\begin{array}{l}\text { High treatment efficiency but much higher cost. Costs reflect } \\
\text { some expensive pilot-scale projects. Simple hydrogeology } \\
\text { makes application easier. Some surfactants/cosolvents can } \\
\text { serve as electron donors for subsequent anaerobic } \\
\text { biodegradation reactions. }\end{array}$ \\
\hline Air sparging & - & - & $\begin{array}{l}\text { May be suitable if only limited biological attenuation is } \\
\text { occurring at a site. Addition of oxygen can disrupt anaerobic } \\
\text { processes that may be occurring. }\end{array}$ \\
\hline $\begin{array}{l}\text { Pump and Treat source } \\
\text { containment }\end{array}$ & NA & & $\begin{array}{l}\text { Due to the high groundwater flow rate, a large system may be } \\
\text { required. This approach does not reduce mass significantly } \\
\text { compared to the rate of mass loss without P\&T and may need } \\
\text { to be operated for a long time. }\end{array}$ \\
\hline
\end{tabular}




\section{SCENARIO NUMBER 3}

\section{Simple Fast Flow} and

\section{Aerobic}
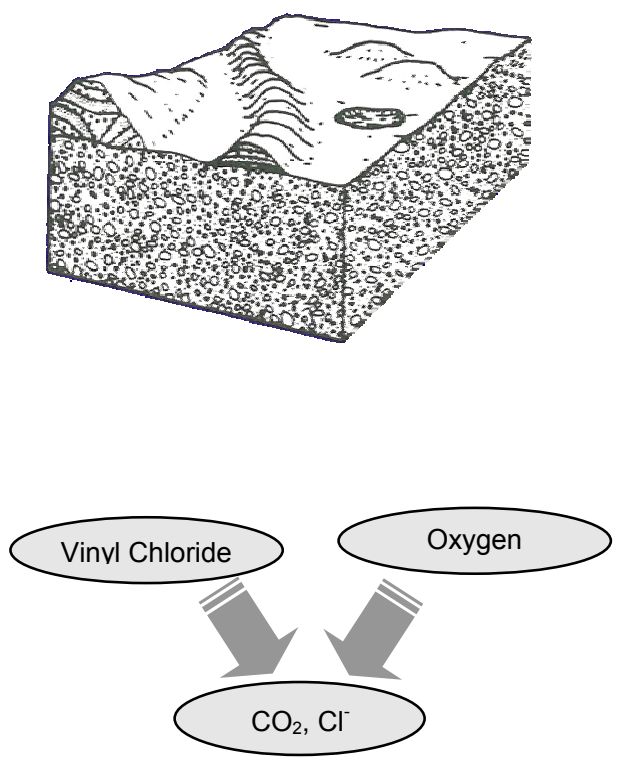


\begin{tabular}{|l|l|l|}
\hline Aerobic & Anoxic & Anaerobic \\
\hline
\end{tabular}

\section{SCENARIO 3 DESCRIPTION: SIMPLE FAST FLOW and AEROBIC}

The hydrogeologic setting and geochemical setting below define the basic scenario type. This scenario has the following characteristics:

\section{Hydrogeologic Setting}

"Simple and Fast " Hydrogeology:

- Only one hydrogeologic unit

- Relatively uniform hydraulic conductivity

- Relatively high groundwater seepage velocity

(see Section 2.1 and Appendix 1 for more information)

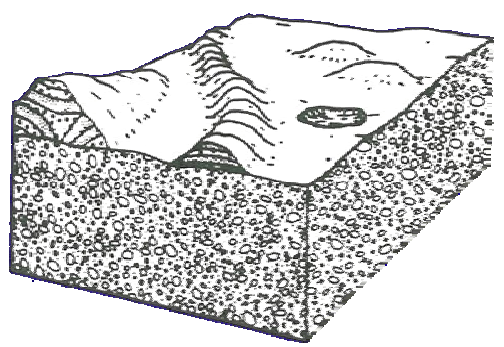

Block Diagram of Outwash Aquifer from DRASTIC System

\section{Geochemical Setting}

"Aerobic" Geochemistry:

- Dissolved oxygen and redox are moderate to high

- Possible to have wide range of concentrations of competing electron acceptors (nitrate, sulfate)

- $\quad$ No or very limited presence of anaerobic indicators (e.g., methane).

(see Section 2.2 for more information)

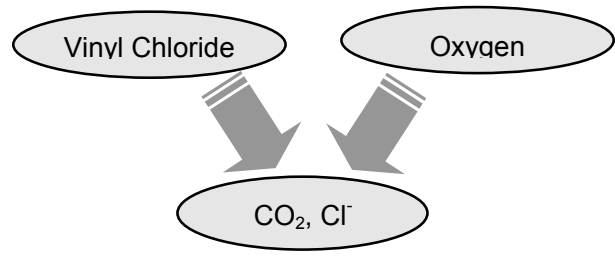

Example Reactions for "Aerobic" Geochemical Setting 


\section{KEY DECHLORINATION REACTIONS}

\section{Reaction Overview}

The chart to the right shows which reactions are likely to occur, which occur but at a slow rate, and which may occur under specific conditions, and which are unlikely to occur.

\section{Compounds Easier for Biological Degradation}

- $\quad$ cis $1,2-\mathrm{DCE}$

- $\mathrm{VC}$

- 1,2-DCA

- 1,1-DCE

- DCM

- $\mathrm{CM}$

\section{Compounds More Difficult for Biological Degradation}

- PCE

- TCE

- $\quad \mathrm{CT}$

- $\mathrm{CF}$

See Section 5.1 for more information about reactions

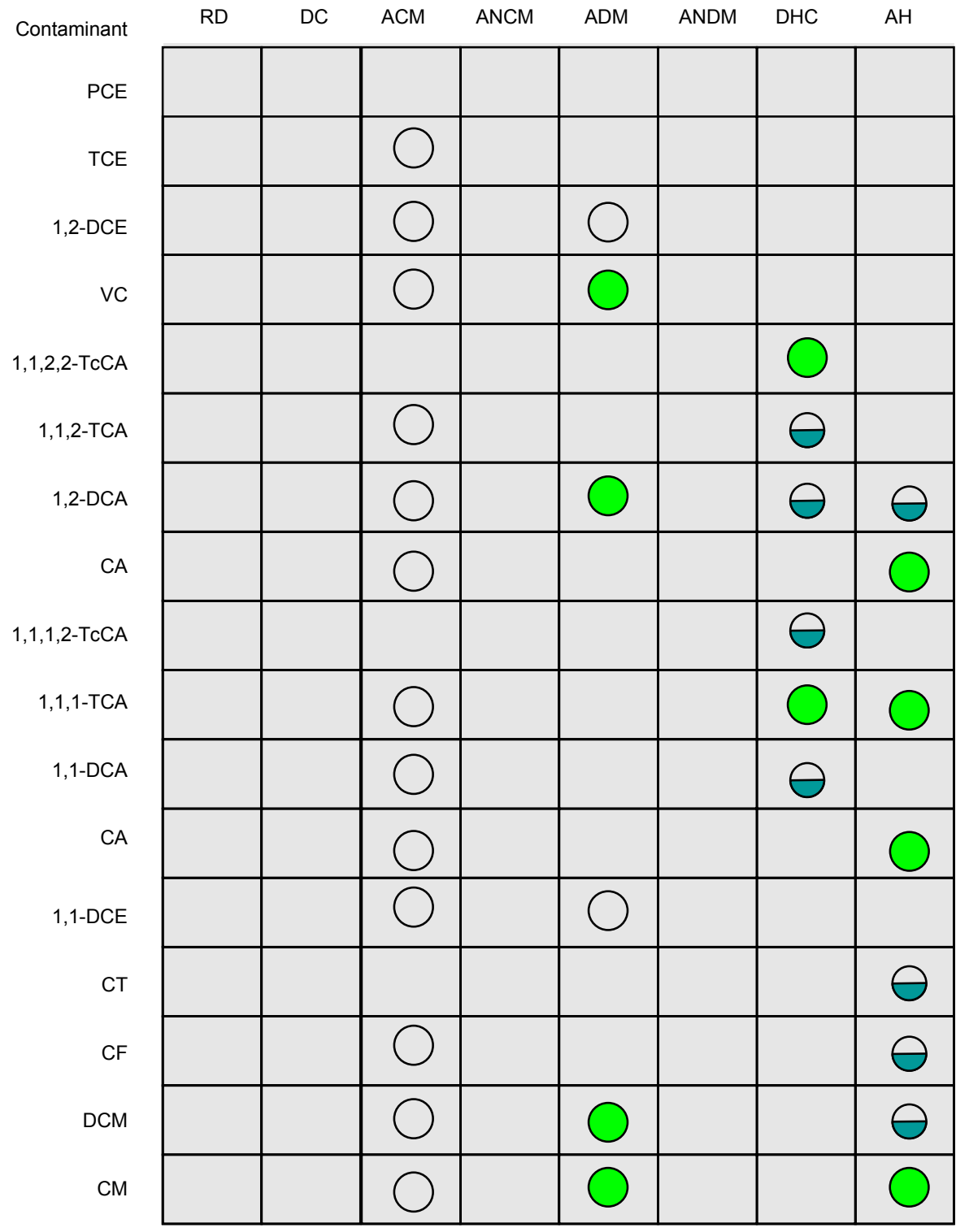

RFACTIONS

Key:

Highly Likely to occur

Highly likely to occur, but a slow rate

May occur under specific conditions

Highly Unlikely to occur

SCENARIO 3 - 3

\begin{tabular}{|c|l|}
\hline ACM & Aerobic Co-Metabolism \\
\hline ANCM & Anaerobic Co-Metabolism \\
\hline ADM & Aerobic Direct Metabolism \\
\hline ANDM & Anaerobic Direct Metabolism \\
\hline DHC & Dehydrochlorination (abiotic) \\
\hline AH & Abiotic Hydrolysis \\
\hline DC & Dichloroelimination (biotic) \\
\hline RD & Reductive Dechlorination (hydrogenolysis) \\
\hline
\end{tabular}




\section{EFFECT OF MODIFYING FACTORS}

\begin{tabular}{|c|c|}
\hline MODIFYING FACTOR & KEY POINTS \\
\hline Source StI & $\begin{array}{l}\text { IF YOU HAVE A STRONG SOURCE: } \\
\text { - Plumes (both parent and daughter compounds) may be longer } \\
\text { - Source zones may persist for longer periods of time } \\
\text { IF YOU HAVE A MODERATE SOURCE: } \\
\text { - Intermediate condition between Strong and Weak Source } \\
\text { IF YOU HAVE A WEAK SOURCE: } \\
\text { - Plumes may be shorter } \\
\text { - Source zones may not persist as long } \\
\text { - MNA alone or MNA with EA more likely to be sufficient }\end{array}$ \\
\hline Source Typ & $\begin{array}{l}\text { IF YOU HAVE MOSTLY A VADOSE ZONE SOURCE: } \\
\text { - Source may appear small due to dilution but can be large } \\
\text { - Sources in clay vadose zones will be weaker but more long-lived than } \\
\text { - } \text { sandy vadose zone } \\
\text { Plumes will be thinner and closer to water table } \\
\text { IF YOU HAVE A SUBMERGED SOURCE: } \\
\text { - Simple, fast hydrogeology means matrix diffusion will be less important } \\
\text { than at slow, complex sites. } \\
\text { - Source mass flux can decrease relatively rapidly as DNAPL fingers } \\
\text { - Pissolve } \\
\text { IF YOU HAVE A MIXED SOURCE: } \\
\text { - May have combination of the factors above }\end{array}$ \\
\hline Travel Time to Recept & $\begin{array}{l}\text { CLOSE RECEPTORS (<2 YEARS TRAVEL TIME) } \\
\text { - More intensive monitoring system likely to be needed due to potential } \\
\text { - } \quad \text { Morious consequences in event of failure of MNA/EA. } \\
\text { MODERATE RECEPTORS (>2 but < } 5 \text { YEARS TRAVEL TIME) } \\
\text { - Represents middle-ground case } \\
\text { DISTANT RECEPTORS ( }>5 \text { YEARS TRAVEL TIME) } \\
\text { - Less intensive monitoring system likely to be needed due to lower } \\
\text { - potential for serious consequences in event of failure of MNA/EA. } \\
\text { MNA alone or MNA with EA more likely to be sufficient }\end{array}$ \\
\hline Plume Stability & $\begin{array}{l}\text { EXPANDING OR PERTURBED' PLUME } \\
-\quad \text { More intensive monitoring system likely to be needed } \\
\text { STABLE PLUME } \\
\text { - Represents middle-ground case } \\
\text { SHRINKING PLUME } \\
\text { - Less intensive monitoring system likely to be needed } \\
\text { - MNA alone likely to be sufficient } \\
{ }^{1} \text { For instance if the plume has been impacted by a previous remedy such as P\&T. }\end{array}$ \\
\hline
\end{tabular}




\begin{tabular}{|c|c|c|c|c|c|c|c|}
\hline $\begin{array}{c}\text { Simple } \\
\text { Fast }\end{array}$ & $\begin{array}{c}\text { Simple } \\
\text { Slow }\end{array}$ & $\begin{array}{c}\text { Heterogeneous } \\
\text { Fast }\end{array}$ & $\begin{array}{c}\text { Heterogeneous } \\
\text { Slow }\end{array}$ & $\begin{array}{c}\text { Fractured/ } \\
\text { Porous Rock }\end{array}$ \\
\hline
\end{tabular}

\section{WILL MNA WORK?}

\section{Potential for MNA Processes to Control Plume}

In fast-flowing homogeneous plumes or plume segments where aerobic conditions are present uniformly throughout the plume, there is less likelihood that natural attenuation processes will result in short, stable or shrinking plumes than in anaerobic plumes or plume segments if parent compounds such as PCE and TCE are present. While TCE and some other parent compounds can be degraded biologically under aerobic conditions, these reactions are cometabolic reactions that require the presence of methane or another similar substrate that are typically not present in aerobic aquifers under natural conditions. Abiotic degradation processes will occur for some compounds, but may produce daughter products that cannot be readily degraded under aerobic conditions. Some compounds can be degraded directly by aerobic bacteria (e.g., DCE and VC). In summary, aerobic conditions are generally less conducive for managing chlorinated solvent plumes, except for a plume segment downgradient of an anaerobic plume segment where the contamination is dominated by reductive dechlorination daughter products such as cis-1,2DCE or VC that can be directly degraded under aerobic conditions.

The fast nature of the hydrogeologic setting means that: i) there will be a high mass flux of oxygen entering the plume segment, so it is less likely that direct biodegradation reactions will be oxygen-limited; and ii) it is more likely that relatively long contaminant plumes will result for compounds which do not degrade readily in aerobic geochemical settings.

\section{Key Sustainability Concept}

Direct aerobic biologic reactions and abiotic reactions are likely to be sustainable indefinitely.

Other biodegradation reactions that can occur under aerobic conditions are co-metabolic reactions that require oxygen and a primary substrate (such as methane). The probability that the supply of dissolved oxygen to the plume from upgradient sources (and plume re-aeration to a lesser degree) will be interrupted is relatively low. However, changes in source structure over time could result in reduced delivery of the primary substrate, increasing the uncertainty in the long-term sustainability of a naturally occurring co-metabolic reaction. 


\begin{tabular}{|l|l|l|}
\hline Aerobic & Anoxic & Anaerobic \\
\hline
\end{tabular}

\section{HOW DO I CHARACTERIZE THIS TYPE OF SITE?}

\section{Actions Needed to Determine MNA Viability:}

The following are key considerations for evaluating the viability of MNA at a Simple Fast Flow and Aerobic type site:

- trends for contaminant concentrations need to be established to assess whether attenuation is occurring (the aerobic setting means that a daughter products will likely not be available to assess whether attenuation processes are occurring);

- $\quad$ confirm that aerobic conditions are present throughout the entire plume/plume segment;

- $\quad$ determine if plume(s) are expanding/perturbed, stable, or shrinking;

- determine the location of any receptors (if present) and determine the travel time from the edge of the plume to these receptors.

\section{Key Monitoring Concepts}

You will likely just need a simple set of transect wells along the plume centerline and some sentry wells - looks like the "text book" case due to the simple plume shape in a homogenous aquifer.

The fast hydrogeologic setting may mean the plume can be relatively large, and therefore require more monitoring points. In addition, the plume can become stable (if it is going to) more quickly than for a slow hydrogeologic setting, so an extremely long temporal record (i.e., the number of years of monitoring data you have) may not be needed to determine plume stability.

\section{Key Uncertainty Concepts}

Because daughter compounds for direct aerobic metabolism of contaminants are not produced and cannot be used to confirm the presence of aerobic reactions, it may be difficult to show that this type of attenuation process is occurring.

It may also be uncertain whether co-metabolic reactions are occurring in the plume segment. To resolve this uncertainty, it may be necessary to perform a detailed analysis of contaminant loss down the centerline of the plume: i) to determine if the observed reduction in concentrations is due to dispersion only or due to a combination of dispersion and co-metabolic reactions; and ii) to determine if a primary substrate (e.g., phenol, methane, propane, etc.) is present in the plume segment. 


\begin{tabular}{|c|c|c|c|c|}
\hline $\begin{array}{c}\text { Simple } \\
\text { Fast }\end{array}$ & $\begin{array}{c}\text { Simple } \\
\text { Slow }\end{array}$ & $\begin{array}{c}\text { Heterogeneous } \\
\text { Fast }\end{array}$ & $\begin{array}{c}\text { Heterogeneous } \\
\text { Slow }\end{array}$ & $\begin{array}{c}\text { Fractured/ } \\
\text { Porous Rock }\end{array}$ \\
\hline
\end{tabular}

\begin{tabular}{|l|l|l|}
\hline Aerobic & Anoxic & Anaerobic \\
\hline
\end{tabular}

\section{HOW DO I ANALYZE DATA?}

In a fast-flowing aquifer, a plume is more likely to show concentration differences over a longer distance and the plume will become stable (if it is going to) in a shorter period of time than in slower-flow aquifers. Thus, a good first step in this type of aquifer is to examine plume maps, concentration vs. time at each well, and concentration vs. distance plots to determine whether the plume is attenuating. Concentrations should show a reduction of contaminant concentrations with distance if attenuation is occurring. Because daughter products are not readily measured for aerobic reactions, additional information to confirm attenuation processes may be needed. Especially if the plume edge is close to receptors, it may be necessary to provide additional data to verify aerobic degradation processes are occurring and to assess the sustainability of these processes. Molecular probe data to verify the presence of the appropriate microorganisms and laboratory microcosm tests may provide this type of information. A more detailed geochemical analysis may also be warranted to assess sustainability. A simple transport model such as BIOCHLOR (used without the sequential decay option) can be helpful in analyzing and visualizing the data and expected plume conditions for comparison to field data. The chart below summarizes an approach for analyzing data at sites depending on whether the concentration data indicates that the plume is decreasing, stable, or increasing and the source type. As noted in the table, as the source gets stronger and the plume is less likely to be decreasing in extent, more information is needed to support selection of an MNA remedy.

\begin{tabular}{|c|c|c|c|}
\hline & \multicolumn{3}{|c|}{ PLUME STATUS } \\
\hline $\begin{array}{l}\text { CONTAMINANT } \\
\text { CONCENTRATIONS/GEOCHEMICAL } \\
\text { STATUS }\end{array}$ & $\begin{array}{c}\text { DECREASING OR } \\
\text { PROBABLY DECREASING }\end{array}$ & STABLE & $\begin{array}{c}\text { INCREASING, } \\
\text { PROBABLY INCREASING, } \\
\text { OR PERTURBED }{ }^{1}\end{array}$ \\
\hline Weak Source & - Mass loss & $\begin{array}{l}\text { - } \quad \text { Mass loss } \\
\text { - } \quad \text { Geochemical footprints }\end{array}$ & $\begin{array}{l}\text { - } \text { Mass loss } \\
\text { - } \text { Geochemical footprints } \\
\text { - Simple Model }\end{array}$ \\
\hline Strong Source & $\begin{array}{l}\text { - } \quad \text { Mass loss } \\
\text { - } \quad \text { Geochemical footprints }\end{array}$ & $\begin{array}{ll}\text { - } & \text { Mass loss } \\
\text { - } & \text { Geochemical footprints } \\
\text { - } & \text { Simple Model }\end{array}$ & $\begin{array}{ll}\text { - } & \text { Mass loss } \\
\text { - } & \text { Geochemical footprints } \\
\text { - } & \text { Comprehensive Model/ } \\
\text { Special Studies }\end{array}$ \\
\hline
\end{tabular}

${ }^{1}$ For instance if the plume has been impacted by a previous remedy such as P\&T.

To demonstrate mass loss construct these graphics:

- Concentration vs. time plots at individual wells;

- Concentration vs. distance plots, with multiple lines for different sampling events through time;

- Plume maps showing plume extent at different times (i.e., either panel maps, or one map with several plume boundaries for different times).

To show geochemical footprints make tables or figures that show:

- Daughter product production from abiotic reactions;

- Presence of primary substrate for co-metabolic reactions;

- Chloride product (this may not work at many sites, however, due to background chloride);

- Moderate to high dissolved oxygen concentrations (shows geochemical conditions are OK);

- No or limited methane production (shows geochemical conditions are OK).

To perform modeling, typical tools include the following:

- Simple transport model (analytical model, e.g., BIOCHLOR, BIOBALANCE ${ }^{1}$ );

- Comprehensive transport model (numerical model, e.g., RT3D).

If a special study is needed, some of the following may be applicable:

- Carbon/chlorine isotope analysis (indicator of degradation processes)

- Molecular probes (indicators of microbial activity)

- Microcosm tests (determine the reaction processes occurring at the site).

${ }^{1}$ Developed by Groundwater Services (www.gsi-net.com), with the support of the Savannah River National Laboratory and $\mathrm{DOE}$, to evaluate monitored natural attenuation at chlorinated solvent sites. 


\begin{tabular}{|c|c|c|c|c|c|c|c|}
\hline $\begin{array}{c}\text { Simple } \\
\text { Fast }\end{array}$ & $\begin{array}{c}\text { Simple } \\
\text { Slow }\end{array}$ & $\begin{array}{c}\text { Heterogeneous } \\
\text { Fast }\end{array}$ & $\begin{array}{c}\text { Heterogeneous } \\
\text { Slow }\end{array}$ & $\begin{array}{c}\text { Fractured/ } \\
\text { Porous Rock }\end{array}$ \\
\hline
\end{tabular}

\section{WHAT ABOUT COSTS AND ENHANCEMENTS?}

Costs for evaluating and implementing MNA for this scenario are primarily dependent on the following items.

- Source strength - Stronger and longer lasting sources will be more costly especially due to the high groundwater flow rate.

- $\quad$ Depth to the plume and size of the plume - Deeper, larger plumes require more and costlier monitoring wells.

- $\quad$ Travel time to the receptor - Plumes closer to receptors will require more frequent monitoring especially with the high groundwater flow rate.

- $\quad$ Plume stability - Less stable plumes require more rigorous evaluation and monitoring to select and implement MNA especially with the high groundwater flow rate.

MNA may be a viable single remedy for the site. If it is determined that MNA may not meet remediation goals, a good first option is to evaluate the potential use of sustainable enhancements (enhanced attenuation). The objective being to adjust the attenuation conditions sufficiently such that the plume is controlled. If the enhancements are insufficient to control the plume, source control treatment may be required. In general, enhanced attenuation is less likely a viable option for a fast flow regime with a strong source, unless source treatment is undertaken initially. The sections below discuss options for enhanced attenuation and source control related to this scenario.

\section{Key Enhanced Attenuation Concepts}

Enhancements are presented organized by the different zones in which they may be applied: source zone (reduction of contaminant mass flux to plume); plume (enhanced attenuation processes); or discharge zone (enhanced attenuation processes). Within the source zone, enhancements can be applied as a hydraulic manipulation or as a passive source reduction (active source control is discussed in the next section). Within the plume and discharge zone, either biological (microbial or plant based) or abiotic (abiotic degradation, reactive barriers, sorption) attenuation processes can be enhanced. A description of potential enhanced attenuation approaches, and their applicability to Scenario 3 sites, is shown below. More detailed information about each technology listed below is available in Early et al., (2005). 


\begin{tabular}{|c|c|c|c|c|}
\hline $\begin{array}{c}\text { Simple } \\
\text { Fast }\end{array}$ & $\begin{array}{c}\text { Simple } \\
\text { Slow }\end{array}$ & $\begin{array}{c}\text { Heterogeneous } \\
\text { Fast }\end{array}$ & $\begin{array}{c}\text { Heterogeneous } \\
\text { Slow }\end{array}$ & $\begin{array}{c}\text { Fractured/ } \\
\text { Porous Rock }\end{array}$ \\
\hline
\end{tabular}

\begin{tabular}{|l|l|l|}
\hline Aerobic & Anoxic & Anaerobic \\
\hline
\end{tabular}

\section{Enhancement Summary}

\begin{tabular}{|c|c|c|}
\hline ENHANCEMENT & DESCRIPTION & APPLICABILITY TO SCENARIO 3 SITES \\
\hline \multicolumn{3}{|c|}{ SOURCE ZONE ENHANCEMENTS } \\
\hline $\begin{array}{l}\text { Surface water or } \\
\text { groundwater } \\
\text { interception/diversion }\end{array}$ & $\begin{array}{l}\text { Use of interception } \\
\text { trenches or wells, surface } \\
\text { covers, or phyto-covers } \\
\text { (plants) to reduce water } \\
\text { flux through source area }\end{array}$ & $\begin{array}{l}\text { May be more difficult in high flow rate conditions. Likely more } \\
\text { applicable using surface covers or phyto-covers if the source is } \\
\text { primarily within the vadose zone. }\end{array}$ \\
\hline Physical containment & $\begin{array}{l}\text { Use of grout walls and } \\
\text { other physical } \\
\text { containment }\end{array}$ & $\begin{array}{l}\text { Potentially applicable depending on the geometry of the source } \\
\text { zone. }\end{array}$ \\
\hline Passive extraction & $\begin{array}{c}\text { Use of passive soil vapor } \\
\text { extraction }\end{array}$ & Useful if the source is primarily within the vadose zone. \\
\hline $\begin{array}{l}\text { Enhanced } \\
\text { biodegradation }\end{array}$ & $\begin{array}{l}\text { Injection of long-term } \\
\text { dissolved oxygen source }\end{array}$ & $\begin{array}{l}\text { Well suited for aerobic sites if oxygen concentrations are marginal } \\
\text { in some areas; enhances existing aerobic biodegradation } \\
\text { reactions. Simple hydrogeology makes application easier. Faster } \\
\text { groundwater flow may require larger amounts of oxygen than in } \\
\text { slower groundwater if electron donors are carried into the } \\
\text { treatment zone. }\end{array}$ \\
\hline $\begin{array}{l}\text { Enhanced } \\
\text { biodegradation }\end{array}$ & $\begin{array}{l}\text { Injection of electron donor } \\
\text { (e.g., HRC, molasses, } \\
\text { vegetable oil) to enhance } \\
\text { microbial degradation of } \\
\text { the source. }\end{array}$ & $\begin{array}{l}\text { Typically more appropriate for anaerobic sites; stimulates } \\
\text { anaerobic contaminant biodegradation reactions. Potentially } \\
\text { useful at aerobic sites in source area to convert contaminants } \\
\text { such as PCE and TCE into contaminants such as DCE and VC } \\
\text { that are degradable under aerobic conditions. Need careful } \\
\text { control of process to avoid depleting all of the oxygen for the } \\
\text { plume and eliminating the potential for aerobic reactions. Simple } \\
\text { hydrogeology makes application easier. Faster groundwater flow } \\
\text { may require larger amounts of electron donor than in slower } \\
\text { groundwater if electron acceptors are carried into the treatment } \\
\text { zone. }\end{array}$ \\
\hline $\begin{array}{l}\text { Permeable reactive } \\
\text { barrier }\end{array}$ & $\begin{array}{l}\text { Use of zero valent iron, } \\
\text { reduced sediment iron, } \\
\text { enhanced partitioning, or } \\
\text { biological barrier to } \\
\text { attenuate some of the } \\
\text { contaminant flux at the } \\
\text { downgradient edge of the } \\
\text { source area. }\end{array}$ & $\begin{array}{l}\text { Barriers typically use anaerobic reactions. Influent of dissolved } \\
\text { oxygen is problematic for the barrier and the aquifer down } \\
\text { gradient of the barrier will be depleted in oxygen. Thus, barriers } \\
\text { that use anaerobic reactions are not typically suitable for aerobic } \\
\text { sites. May be more difficult and expensive to construct due to fast } \\
\text { flow regime. Faster groundwater flows potentially result in more } \\
\text { contaminant and competing electron acceptors passing through } \\
\text { the barrier, requiring greater thickness to achieve desired } \\
\text { treatment levels. }\end{array}$ \\
\hline
\end{tabular}




\begin{tabular}{|c|c|c|c|c|}
\hline $\begin{array}{c}\text { Simple } \\
\text { Fast }\end{array}$ & $\begin{array}{c}\text { Simple } \\
\text { Slow }\end{array}$ & $\begin{array}{c}\text { Heterogeneous } \\
\text { Fast }\end{array}$ & $\begin{array}{c}\text { Heterogeneous } \\
\text { Slow }\end{array}$ & $\begin{array}{c}\text { Fractured/ } \\
\text { Porous Rock }\end{array}$ \\
\hline
\end{tabular}

\begin{tabular}{|l|c|c|}
\hline \multicolumn{2}{|c|}{ GEOCHEMICAL SETTING } \\
\hline Aerobic & Anoxic & Anaerobic \\
\hline
\end{tabular}

\section{Enhancement Summary (Con'td)}

\begin{tabular}{|c|c|c|}
\hline ENHANCEMENT & DESCRIPTION & APPLICABILITY TO SCENARIO 3 SITES \\
\hline \multicolumn{3}{|c|}{ PLUME AND DISCHARGE ZONE ENHANCEMENTS } \\
\hline $\begin{array}{l}\text { Enhanced } \\
\text { biodegradation }\end{array}$ & $\begin{array}{l}\text { Injection of long-term } \\
\text { dissolved oxygen source }\end{array}$ & $\begin{array}{l}\text { Well suited for aerobic sites if oxygen concentrations are marginal } \\
\text { in some areas; enhances existing aerobic biodegradation reactions. } \\
\text { Simple hydrogeology makes application easier. Faster } \\
\text { groundwater flow may require larger amounts of oxygen than in } \\
\text { slower groundwater if electron donors are carried into the treatment } \\
\text { zone. }\end{array}$ \\
\hline $\begin{array}{l}\text { Enhanced } \\
\text { biodegradation }\end{array}$ & $\begin{array}{l}\text { Injection of electron donor } \\
\text { (e.g., HRC, molasses, } \\
\text { vegetable oil) to enhance } \\
\text { microbial degradation of } \\
\text { the source. }\end{array}$ & $\begin{array}{l}\text { Typically more appropriate for anaerobic sites; stimulates } \\
\text { anaerobic contaminant biodegradation reactions. Potentially useful } \\
\text { at aerobic sites in source area to convert contaminants such as } \\
\text { PCE and TCE into contaminants such as DCE and VC that are } \\
\text { degradable under aerobic conditions. Need careful control of } \\
\text { process to avoid depleting all of the oxygen for the plume and } \\
\text { eliminating the potential for aerobic reactions. Simple } \\
\text { hydrogeology makes application easier. Faster groundwater flow } \\
\text { may require larger amounts of electron donor than in slower } \\
\text { groundwater if electron acceptors are carried into the treatment } \\
\text { zone. }\end{array}$ \\
\hline $\begin{array}{l}\text { Permeable reactive } \\
\text { barrier }\end{array}$ & $\begin{array}{l}\text { Use of zero valent iron, } \\
\text { reduced sediment iron, } \\
\text { enhanced partitioning, or } \\
\text { biological barrier to } \\
\text { attenuate some of the } \\
\text { contaminant flux within the } \\
\text { plume. }\end{array}$ & $\begin{array}{l}\text { Barriers typically use anaerobic reactions. Influent of dissolved } \\
\text { oxygen is problematic for the barrier and the aquifer down gradient } \\
\text { of the barrier will be depleted in oxygen. Thus, barriers that use } \\
\text { anaerobic reactions are not typically suitable for aerobic sites. May } \\
\text { be more difficult and expensive to construct due to fast flow regime. } \\
\text { Faster groundwater flows potentially result in more contaminant } \\
\text { and competing electron acceptors passing through the barrier, } \\
\text { requiring greater thickness to achieve desired treatment levels. }\end{array}$ \\
\hline Phytoextraction & $\begin{array}{l}\text { Use of plants to extract } \\
\text { contaminants from near } \\
\text { surface groundwater }\end{array}$ & May be less effective for fast groundwater flow conditions. \\
\hline $\begin{array}{l}\text { Plant-based hydraulic } \\
\text { control (plume } \\
\text { enhancement only) }\end{array}$ & $\begin{array}{l}\text { Use of plants to control } \\
\text { hydraulic gradient and slow } \\
\text { groundwater }\end{array}$ & May be less effective for fast groundwater flow conditions. \\
\hline
\end{tabular}




\begin{tabular}{|c|c|c|c|c|}
\hline $\begin{array}{c}\text { Simple } \\
\text { Fast }\end{array}$ & $\begin{array}{c}\text { Simple } \\
\text { Slow }\end{array}$ & $\begin{array}{c}\text { Heterogeneous } \\
\text { Fast }\end{array}$ & $\begin{array}{c}\text { Heterogeneous } \\
\text { Slow }\end{array}$ & $\begin{array}{c}\text { Fractured/ } \\
\text { Porous Rock }\end{array}$ \\
\hline
\end{tabular}

\begin{tabular}{|l|c|c|}
\hline \multicolumn{2}{|c|}{ GEOCHEMICAL SETTING } \\
\hline Aerobic & Anoxic & Anaerobic \\
\hline
\end{tabular}

\section{Key Source Control Concepts}

A description of potential source control measures, and their applicability to Scenario 3 sites, is shown below. Note that source control measures are unlikely to achieve complete restoration at a site, and some source material is always left behind after treatment (U.S. EPA, 2003; McGuire et al., 2006).

\begin{tabular}{|c|c|c|c|}
\hline Example Technology & $\begin{array}{l}\text { Performance } \\
\left(25^{\text {th }}-75^{\text {th }} \text { Percentile }\right. \\
\% \text { reduction in parent } \\
\text { compound })^{1}\end{array}$ & $\begin{array}{l}\frac{\text { Unit Cost }}{25^{\text {th }}-75^{\text {th }}} \\
\text { Percentile } \\
\text { (\$/yrd) })^{2}\end{array}$ & Applicability to Scenario 3 Sites \\
\hline In-situ biodegradation & $73-99$ & $27-152$ & $\begin{array}{l}\text { Potentially well suited for aerobic sites through addition } \\
\text { of co-substrate for aerobic degradation or potentially } \\
\text { through use of anaerobic reactions depending on how } \\
\text { this action impacts the downgradient geochemical } \\
\text { conditions. Simple hydrogeology makes application } \\
\text { easier. Faster groundwater flow may require larger } \\
\text { amounts of electron donor than in slower groundwater if } \\
\text { electron acceptors are carried into the treatment zone. } \\
\text { Least expensive treatment option. }\end{array}$ \\
\hline Thermal treatment & $68-99.9$ & $48-129$ & Does not appear to disrupt MNA after treatment. \\
\hline Chemical oxidation & $70-97$ & $47-194$ & $\begin{array}{l}\text { Removes more total CVOCs than enhanced } \\
\text { biodegradation but shows more rebound }{ }^{1} \text {. Simple } \\
\text { hydrogeology makes application easier. May be } \\
\text { suitable for aerobic sites. }\end{array}$ \\
\hline Surfactant/cosolvents & $92-98$ & $118-1322$ & $\begin{array}{l}\text { High treatment efficiency but much higher cost. Costs } \\
\text { reflect some expensive pilot-scale projects. Simple } \\
\text { hydrogeology makes application easier. Some } \\
\text { surfactants/cosolvents can serve as electron donors for } \\
\text { subsequent anaerobic biodegradation reactions. }\end{array}$ \\
\hline Air sparging & - & - & $\begin{array}{l}\text { May be a viable alternative depending on the site } \\
\text { geology (e.g., contamination in an unconfined aquifer). }\end{array}$ \\
\hline $\begin{array}{l}\text { Pump and Treat source } \\
\text { containment }\end{array}$ & NA & & $\begin{array}{l}\text { Due to the high groundwater flow rate, a large system } \\
\text { may be required. This approach does not reduce mass } \\
\text { significantly compared to the rate of mass loss without } \\
\text { P\&T and may need to be operated for a long time. }\end{array}$ \\
\hline
\end{tabular}




\begin{tabular}{|c|c|}
\hline $\begin{array}{c}\text { Heterogeneous } \\
\text { Slow }\end{array}$ & $\begin{array}{c}\text { Fractured/ } \\
\text { Porous Rock }\end{array}$ \\
\hline
\end{tabular}

\begin{tabular}{|l|l|l|}
\hline Aerobic & Anoxic & Anaerobic \\
\hline
\end{tabular}

\section{SCENARIO NUMBER 4}

\section{Simple Slow Flow}

and

\section{Anaerobic}
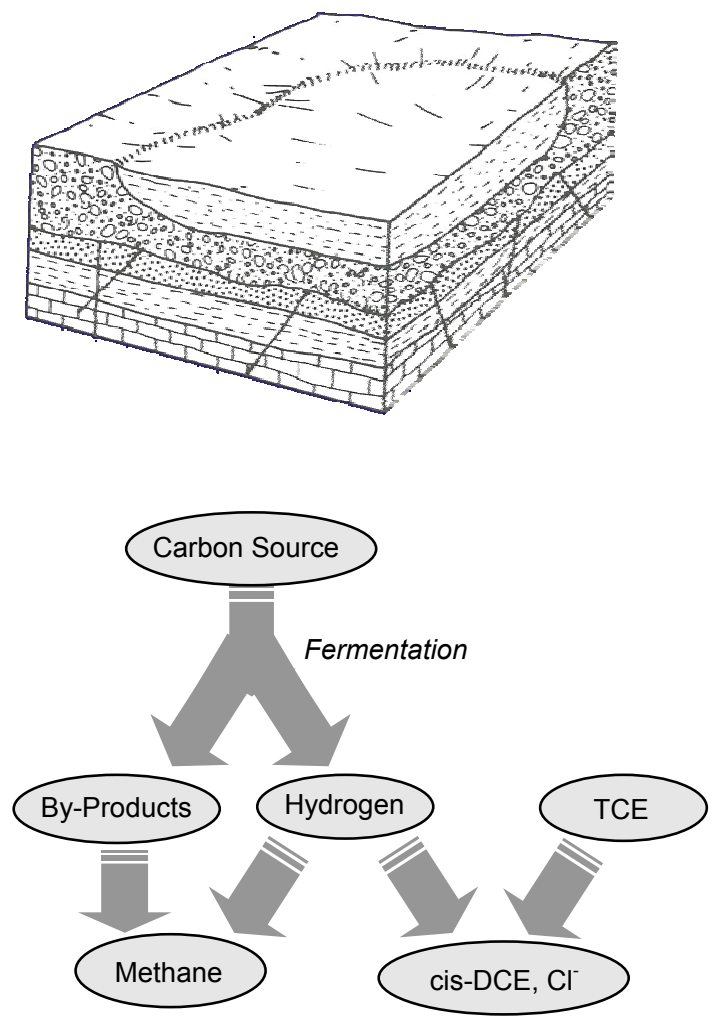


\section{SCENARIO 1 DESCRIPTION: SIMPLE SLOW FLOW and ANAEROBIC}

The hydrogeologic setting and geochemical setting below define the basic scenario type. This scenario has the following characteristics:

\section{Hydrogeologic Setting}

"Simple and Slow " Hydrogeology:

- Only one hydrogeologic unit

- Relatively uniform hydraulic conductivity

- Relatively low groundwater seepage velocity

(see Section 2.1 and Appendix 1 for more information)

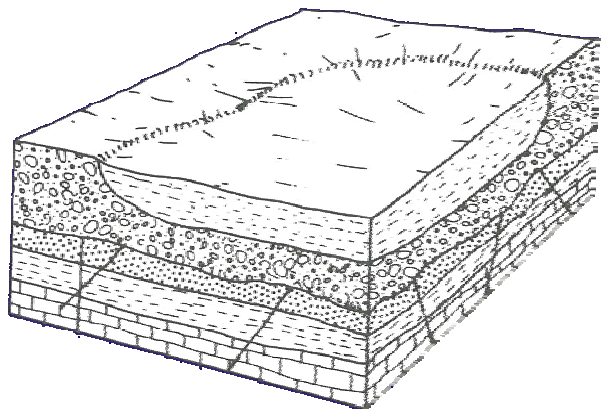

Block Diagram of Glacial Lake Deposit Aquifer from DRASTIC System

\section{Geochemical Setting}

"Anaerobic" Geochemistry:

- Dissolved oxygen and redox are low

- Low to moderate concentrations of competing electron acceptors (nitrate, sulfate)

- Methane being produced.

(see Section 2.2 for more information)
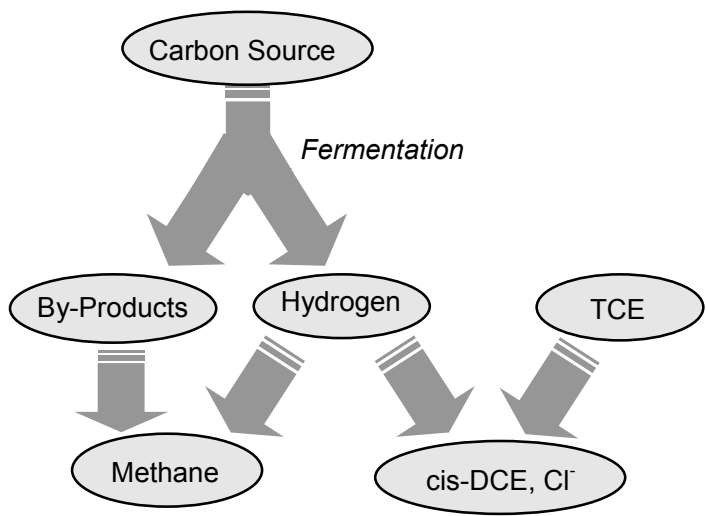

Example Reactions for "Anaerobic" Geochemical Setting 


\section{KEY DECHLORINATION REACTIONS}

\section{Reaction Overview}

The chart to the right shows which reactions are likely to occur, which occur but at a slow rate, which may occur under specific conditions, and which are unlikely to occur.

\section{Typically Biodegradable Parent Compounds}

These compounds are typically degradable under anaerobic conditions:

- PCE

- TCE

- 1,1,1-TCA

- 1,2-DCA

- $\mathrm{CT}$

\section{Typical Daughter Products}

Daughter products that may be present depending on the parent compound and the reactions listed to the right:

- TCE

- $\quad$ cis 1,2-DCE

- VC

- 1,2-DCA

- 1,1-DCE

- $\mathrm{CF}$

See Section 5.1 for more information about reactions

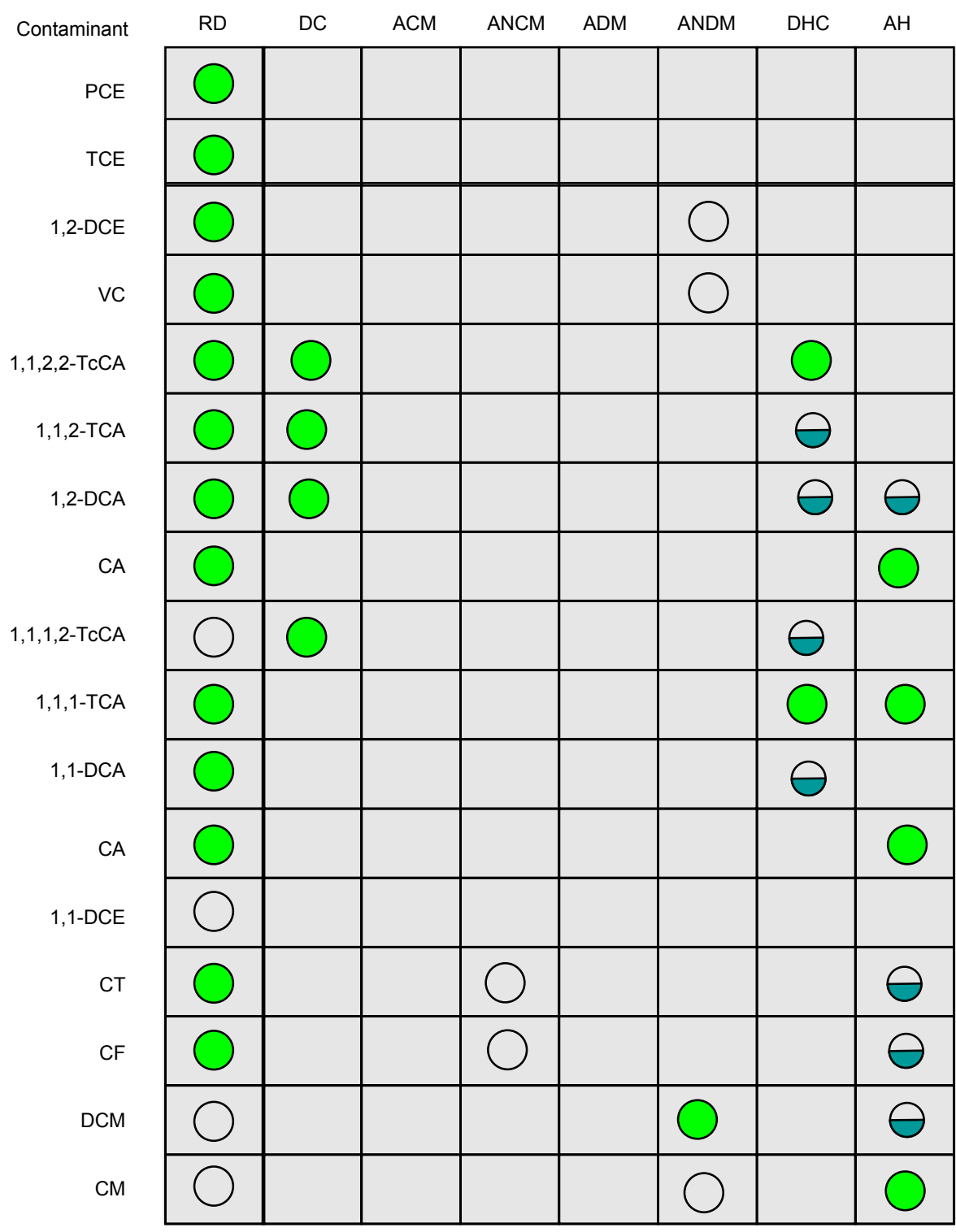

REACTIONS

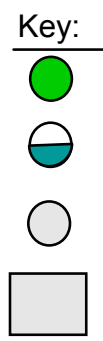

Highly Likely to occur

Highly likely to occur, but a slow rate

May occur under specific conditions

Highly Unlikely to occur

Reactions 


\section{EFFECT OF MODIFYING FACTORS}

\begin{tabular}{|c|c|}
\hline MODIFYING FACTOR & KEY POINTS \\
\hline Source Strength & $\begin{array}{l}\text { IF YOU HAVE A STRONG SOURCE: } \\
\text { - Plumes (both parent and daughter compounds) may be longer } \\
\text { - } \quad \text { Source zones may persist for longer periods of time } \\
\text { - } \quad \text { More likely to need EA or source control measures } \\
\text { IF YOU HAVE A MODERATE SOURCE: } \\
\text { - Intermediate condition between Strong and Weak Source } \\
\text { IF YOU HAVE A WEAK SOURCE: } \\
\text { - Plumes may be shorter } \\
\text { - } \quad \text { Source zones may not persist as long } \\
\text { - MNA alone or MNA with EA more likely to be sufficient }\end{array}$ \\
\hline Source & $\begin{array}{l}\text { IF YOU HAVE MOSTLY A VADOSE ZONE SOURCE: } \\
\text { - Source may appear small due to dilution but can be large } \\
\text { - } \quad \text { Sources in clay vadose zones will be weaker but more long-lived than } \\
\text { sandy vadose zone } \\
\text { - Plumes will be thinner and closer to water table } \\
\text { IF YOU HAVE A SUBMERGED SOURCE: } \\
\text { - Simple, slow hydrogeology means matrix diffusion may be important, but } \\
\text { - less important than at complex sites. } \\
\text { - Source mass flux will decrease as DNAPL fingers dissolve } \\
\text { IF YOU HAVE A MIXED SOURCE: } \\
\text { - } \quad \text { May have combination of the factors above }\end{array}$ \\
\hline Travel Time to $R e$ & $\begin{array}{l}\text { CLOSE RECEPTORS (<2 YEARS TRAVEL TIME) } \\
\text { - More intensive monitoring system likely to be needed due to potential } \\
\text { - } \\
\text { Morious consequences in event of failure of MNA/EA. } \\
\text { MODERATE RECEPTORS (>2 but < } 5 \text { YEARS TRAVEL TIME) } \\
\text { - Represents middle-ground case } \\
\text { DISTANT RECEPTORS ( }>5 \text { YEARS TRAVEL TIME) } \\
\text { - Less intensive monitoring system likely to be needed due to lower } \\
\text { potential for serious consequences in event of failure of MNA/EA. } \\
\text { MNA alone or MNA with EA more likely to be sufficient }\end{array}$ \\
\hline Plume Stability & $\begin{array}{l}\text { EXPANDING OR PERTURBED }{ }^{1} \text { PLUME } \\
\text { - More intensive monitoring system likely to be needed } \\
\text { STABLE PLUME } \\
\text { - Represents middle-ground case } \\
\text { SHRINKING PLUME } \\
\text { - Less intensive monitoring system likely to be needed } \\
\text { MNA alone likely to be sufficient } \\
{ }^{1} \text { For instance if the plume has been impacted by a previous remedy such as P\&T. }\end{array}$ \\
\hline
\end{tabular}




\begin{tabular}{|c|c|c|c|c|}
\hline $\begin{array}{c}\text { Simple } \\
\text { Fast }\end{array}$ & $\begin{array}{c}\text { Simple } \\
\text { Slow }\end{array}$ & $\begin{array}{c}\text { Heterogeneous } \\
\text { Fast }\end{array}$ & $\begin{array}{c}\text { Heterogeneous } \\
\text { Slow }\end{array}$ & $\begin{array}{c}\text { Fractured/ } \\
\text { Porous Rock }\end{array}$ \\
\hline
\end{tabular}

\begin{tabular}{|l|l|l|}
\hline Aerobic & Anoxic & Anaerobic \\
\hline
\end{tabular}

\section{WILL MNA WORK?}

\section{Potential for MNA Processes to Control Plume}

This scenario often is well suited for natural attenuation processes to manage the contaminants in the plume or plume segment. The anaerobic conditions almost always mean that biodegradation processes are active.

In a slow-flowing heterogeneous aquifer where anaerobic conditions are present uniformly throughout the plume, relatively low rates of contaminant degradation can stabilize the plume. With the typical reductive dechlorination processes that are occurring under these conditions, it would be expected that the daughter product plume would be larger than the parent product plume because the degradation rate of daughters is typically slower than the rate for the parents.

At some Scenario 4 sites, "DCE stall" may be of concern. DCE stall is an informal term typically used to describe conditions at chlorinated ethene sites where the cis-1,2-DCE "stalls outs" or exhibits a very low conversion rate to VC. This DCE "stall" condition has been ascribed to a variety of factors, including:

- $\quad$ Lack of the necessary microbiological communities that are required to degrade cis-1,2-DCE to VC;

- The direct conversion of cis-1,2-DCE to carbon dioxide, which makes it appear that cis-1,2-DCE is not being biodegraded because VC is not being produced; but in fact the cis-1,2-DCE is being biodegraded by direct oxidation to carbon dioxide;

- Conditions which are anaerobic enough to support the conversion of TCE to cis-1,2-DCE but not anaerobic enough to support the conversion from cis-1,2-DCE to VC by reductive dechlorination;

- $\quad$ Toxicity effects caused at sites where sulfate reducers are producing hydrogen sulfide $\left(\mathrm{H}_{2} \mathrm{~S}\right)$, but the $\mathrm{H}_{2} \mathrm{~S}$ is not being precipitated fast enough by ferrous iron (a by-product of ferric iron reduction) to prevent toxicity effects in the cis-1,2-DCE degraders.

While the cause of cis-1,2-DCE stall is still being evaluated by a number of researchers, the main implication is that at some chlorinated ethene sites, cis-1,2-DCE plumes are expanding and not being controlled. DCE "stall" does not affect long-term sustainability of a reaction, but does determine if natural attenuation processes are sufficient to prevent migration of the plume.

\section{Key Sustainability Concept}

Slow-flowing plume segments may have only low or moderate mass flux of contaminants leaving the source, and therefore moderate rates of degradation are often sufficient to attenuate the plume. Sufficient natural substrate (e.g., organic matter) or co-contaminants that serve as electron donors and can act as a substrate are needed to sustain anaerobic degradation. The BIOBALANCE software system ${ }^{1}$ has a module designed to evaluate sustainability issues for anaerobic MNA reactions. Key input data are: i) mass fraction of solvents vs. donors in NAPL; OR ii) dissolvedphase concentrations of solvents and donors in the source zone.

1 Developed by Groundwater Services (www.gsi-net.com), with the support of the Savannah River National Laboratory and DOE, to evaluate monitored natural attenuation at chlorinated solvent sites. 


\begin{tabular}{|c|c|}
\hline $\begin{array}{c}\text { Heterogeneous } \\
\text { Slow }\end{array}$ & $\begin{array}{c}\text { Fractured/ } \\
\text { Porous Rock }\end{array}$ \\
\hline
\end{tabular}

\begin{tabular}{|l|l|l|}
\hline Aerobic & Anoxic & Anaerobic \\
\hline
\end{tabular}

\section{HOW DO I CHARACTERIZE THIS TYPE OF SITE?}

\section{Actions Needed to Determine MNA Viability}

The following are key considerations for evaluating the viability of MNA at a Simple Slow Flow and Anaerobic type site:

- both parent compound and daughter compounds need to be delineated (the anaerobic setting means that a number of daughter products will likely be generated);

- confirm that anaerobic conditions are present throughout the entire plume segment;

- determine if plume(s) are expanding/perturbed, stable, or shrinking;

- determine the location of any receptors (if present) and determine the travel time from the edge of the plume to these receptors;

- a mass-balance type evaluation of the source zone will help to determine if the electron donor supply is sustainable over the long term.

\section{Key Monitoring Concepts}

You will likely need a simple set of transect wells along the plume centerline and some sentry wells - looks like the "text book" case due to the simple plume shape in a homogenous aquifer.

The slow hydrogeologic setting may mean the plume is of moderate size. However, a long period of time may be required for the plume to become stable (if it is going to). Thus a long temporal record (i.e., the number of years of monitoring data you have) may be needed to determine plume stability.

\section{Key Uncertainty Concepts}

There may be uncertainty about the plume conditions in general under this scenario because the plume may not be fully developed due to the slow flow conditions and it may not be evident if MNA is currently working. Sustainability of MNA may be an issue under anaerobic degradation conditions depending on the mass flux of the source (see key sustainability concept section). 


\begin{tabular}{|c|c|c|c|c|}
\hline $\begin{array}{c}\text { Simple } \\
\text { Fast }\end{array}$ & $\begin{array}{c}\text { Simple } \\
\text { Slow }\end{array}$ & $\begin{array}{c}\text { Heterogeneous } \\
\text { Fast }\end{array}$ & $\begin{array}{c}\text { Heterogeneous } \\
\text { Slow }\end{array}$ & $\begin{array}{c}\text { Fractured/ } \\
\text { Porous Rock }\end{array}$ \\
\hline
\end{tabular}

\begin{tabular}{|l|l|l|}
\hline Aerobic & Anoxic & Anaerobic \\
\hline
\end{tabular}

\section{HOW DO I ANALYZE DATA?}

In a slow-flowing aquifer, a plume is more likely to show concentration differences over short distances and the plume will become stable (if it is going to) over a long period of time. Thus, while a good first step in this type of aquifer is to examine plume maps, concentration vs. time at each well, and concentration vs. distance plots to determine whether the plume is attenuating, additional information may be needed. A simple transport model such as BIOCHLOR can be helpful in analyzing and visualizing the data and expected plume conditions for comparison to field data. The chart below summarizes an approach for analyzing data at sites depending on whether the concentration data indicates that the plume is decreasing, stable, or increasing and the source type. As noted in the table, as the source gets stronger and the plume is less likely to be decreasing in extent, more information is needed to support selection of an MNA remedy. If the plume stability is not known due to the slow flow conditions and a short temporal monitoring record, it may be necessary to use the types of analysis shown under the "increasing or probably increasing" column in the table.

\begin{tabular}{|c|c|c|c|}
\hline & \multicolumn{3}{|c|}{ PLUME STATUS } \\
\hline $\begin{array}{l}\text { CONTAMINANT } \\
\text { CONCENTRATIONS/GEOCHEMICAL } \\
\text { STATUS }\end{array}$ & $\begin{array}{c}\text { DECREASING OR } \\
\text { PROBABLY DECREASING }\end{array}$ & STABLE & $\begin{array}{l}\text { INCREASING, } \\
\text { PROBABLY INCREASING, } \\
\text { OR PERTURBED }^{1}\end{array}$ \\
\hline Weak Source & - Mass loss & $\begin{array}{l}\text { - } \text { Mass loss } \\
\text { - } \quad \text { Geochemical footprints }\end{array}$ & $\begin{array}{l}\text { - } \text { Mass loss } \\
\text { - } \quad \text { Geochemical footprints } \\
\text { - } \quad \text { Simple Model }\end{array}$ \\
\hline Strong Source & $\begin{array}{l}\text { - } \quad \text { Mass loss } \\
\text { - } \quad \text { Geochemical footprints }\end{array}$ & $\begin{array}{ll}\text { - } & \text { Mass loss } \\
\text { - } & \text { Geochemical footprints } \\
\text { - } & \text { Simple Model }\end{array}$ & $\begin{array}{ll}\text { - } & \text { Mass loss } \\
\text { - } & \text { Geochemical footprints } \\
\text { - } & \text { Comprehensive Model/ } \\
& \text { Special Studies }\end{array}$ \\
\hline
\end{tabular}

${ }^{1}$ For instance if the plume has been impacted by a previous remedy such as P\&T.

To demonstrate mass loss construct these graphics:

- Concentration vs. time plots at individual wells;

- Concentration vs. distance plots, with multiple lines for different sampling events through time;

- Plume maps showing plume extent at different times (i.e., either panel maps, or one map with several plume boundaries for different times).

To show geochemical footprints make tables or figures that show:

- Daughter product production;

- Ethene/Ethane production;

- Chloride production (this may not work at many sites, however, due to background chloride);

- Low dissolved oxygen (shows geochemical conditions are ok);

- Methane and iron(II) distribution (indicators of anaerobic activity);

- Nitrate and sulfate distribution (indicators of competing electron acceptors);

To perform modeling, typical tools include the following:

- Simple transport model (analytical model, e.g., BIOCHLOR, BIOBALANCE ${ }^{1}$ );

- Comprehensive transport model (numerical model, e.g., RT3D).

If a special study is needed, some of the following may be applicable:

- Carbon/chlorine isotope analysis (indicator of degradation processes)

- Molecular probes (indicators of microbial activity)

- Microcosm tests (determine the reaction processes occurring at the site).

1 Developed by Groundwater Services (www.gsi-net.com), with the support of the Savannah River National Laboratory and $\mathrm{DOE}$, to evaluate monitored natural attenuation at chlorinated solvent sites. 


\begin{tabular}{|c|c|c|c|c|}
\hline $\begin{array}{c}\text { Simple } \\
\text { Fast }\end{array}$ & $\begin{array}{c}\text { Simple } \\
\text { Slow }\end{array}$ & $\begin{array}{c}\text { Heterogeneous } \\
\text { Fast }\end{array}$ & $\begin{array}{c}\text { Heterogeneous } \\
\text { Slow }\end{array}$ & $\begin{array}{c}\text { Fractured/ } \\
\text { Porous Rock }\end{array}$ \\
\hline
\end{tabular}

\begin{tabular}{|l|l|l|}
\hline Aerobic & Anoxic & Anaerobic \\
\hline
\end{tabular}

\section{WHAT ABOUT COSTS AND ENHANCEMENTS?}

Costs for evaluating and implementing MNA for this scenario are primarily dependent on the following items.

- $\quad$ Source strength - Stronger and longer lasting sources will be more costly.

- $\quad$ Depth to the plume and size of the plume - Deeper, larger plumes require more and costlier monitoring wells.

- $\quad$ Travel time to the receptor - plumes closer to receptors will require more frequent monitoring and over a long period of time with the slow groundwater flow rate.

- $\quad$ Plume stability - Less stable plumes require more rigorous evaluation and monitoring to select and implement MNA. With the slow groundwater flow rate, more rigorous evaluation or a longer period of monitoring may cause higher costs than for sites with a high groundwater flow rate.

MNA may be a viable single remedy for the site. If it is determined that MNA may not meet remediation goals, a good first option is to evaluate the potential use of sustainable enhancements (enhanced attenuation). The objective being to adjust the attenuation conditions sufficiently such that the plume is controlled. If the enhancements are insufficient to control the plume, source control treatment may be required. In general, enhanced attenuation is less likely a viable option for a fast flow regime with a strong source, unless source treatment is undertaken initially. The sections below discuss options for enhanced attenuation and source control related to this scenario.

\section{Key Enhanced Attenuation Concepts}

Enhancements are presented organized by the different zones in which they may be applied: source zone (reduction of contaminant mass flux to plume); plume (enhanced attenuation processes); or discharge zone (enhanced attenuation processes). Within the source zone, enhancements can be applied as a hydraulic manipulation or as a passive source reduction (active source control is discussed in the next section). Within the plume and discharge zone, either biological (microbial or plant based) or abiotic (abiotic degradation, reactive barriers, sorption) attenuation processes can be enhanced. A description of potential enhanced attenuation approaches, and their applicability to Scenario 4 sites, is shown below. More detailed information about each technology listed below is available in Early et al., (2005). 


\begin{tabular}{c|c|c|c|c}
\hline $\begin{array}{c}\text { Simple } \\
\text { Fast }\end{array}$ & $\begin{array}{c}\text { Simple } \\
\text { Slow }\end{array}$ & $\begin{array}{c}\text { Heterogeneous } \\
\text { Fast }\end{array}$ & $\begin{array}{c}\text { Heterogeneous } \\
\text { Slow }\end{array}$ & $\begin{array}{c}\text { Fractured/ } \\
\text { Porous Rock }\end{array}$ \\
\hline
\end{tabular}

\begin{tabular}{|l|l|l|}
\hline Aerobic & Anoxic & Anaerobic \\
\hline
\end{tabular}

\section{Enhancement Summary}

\begin{tabular}{|c|c|}
\hline Enhancement & Description \\
\hline \multicolumn{2}{|c|}{ SOURCE ZONE ENHANCEMENTS } \\
\hline $\begin{array}{l}\text { Surface water or } \\
\text { groundwater } \\
\text { interception/diversion }\end{array}$ & $\begin{array}{l}\text { Use of interception } \\
\text { trenches or wells, surface } \\
\text { covers, or phyto-covers } \\
\text { (plants) to reduce water } \\
\text { flux through source area } \\
\text { and/or divert unwanted } \\
\text { electron acceptors. }\end{array}$ \\
\hline Physical containment & $\begin{array}{l}\text { Use of grout walls and } \\
\text { other physical } \\
\text { containment }\end{array}$ \\
\hline Passive extraction & $\begin{array}{c}\text { Use of passive soil vapor } \\
\text { extraction }\end{array}$ \\
\hline $\begin{array}{l}\text { Enhanced } \\
\text { biodegradation }\end{array}$ & $\begin{array}{l}\text { Injection of electron donor } \\
\text { (e.g., HRC, molasses, } \\
\text { vegetable oil) to enhance } \\
\text { microbial degradation of } \\
\text { the source. }\end{array}$ \\
\hline $\begin{array}{l}\text { Permeable reactive } \\
\text { barrier }\end{array}$ & $\begin{array}{l}\text { Use of zero valent iron, } \\
\text { reduced sediment iron, } \\
\text { enhanced partitioning, or } \\
\text { biological barrier to } \\
\text { attenuate some of the } \\
\text { contaminant flux at the } \\
\text { downgradient edge of the } \\
\text { source area. }\end{array}$ \\
\hline
\end{tabular}

\section{PLUME AND DISCHARGE ZONE ENHANCEMENTS}

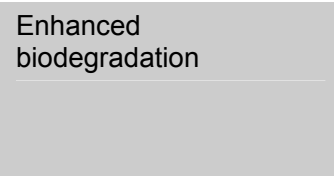

Permeable reactive barrier
Injection of electron donor (e.g., HRC, molasses, vegetable oil) to enhance microbial degradation of the source.

Use of zero valent iron, reduced sediment iron, enhanced partitioning, or biological barrier to attenuate some of the the plume.

\section{Phytoextraction}

Plant-based hydraulic control (plume enhancement only)

Use of plants to extract contaminants from near surface groundwater

Use of plants to control hydraulic gradient and slow groundwater contaminant flux within

Applicability to Scenario 4 Sites

Likely more applicable using surface covers or phytocovers if the source is primarily within the vadose zone. With slow flow rate, this method may be relatively effective compared to application in higher flow rate conditions.

Potentially applicable depending on the geometry of the source zone.

Useful if the source is primarily within the vadose zone.

Well suited for anaerobic sites; enhances existing biodegradation reactions. Suitable for most Scenario 4 sites. Simple hydrogeology makes application easier. Slow groundwater flow may make application easier than for high flow rate conditions.

Easier to apply in slow flow regime. Slower groundwater flows potentially result in less contaminant and competing electron acceptors passing through the barrier, requiring less thickness to achieve desired treatment levels. Anaerobic conditions are helpful because oxygen concentration is low and will not disrupt barriers using anaerobic reactions.

Well suited for anaerobic sites; enhances existing biodegradation reactions. Suitable for most Scenario 4 sites. Simple hydrogeology makes application easier. Slow groundwater flow may make application easier than for high flow rate conditions.

Easier to apply in slow flow regime. Slower groundwater flows potentially result in less contaminant and competing electron acceptors passing through the barrier, requiring less thickness to achieve desired treatment levels. Anaerobic conditions are helpful because oxygen concentration is low and will not disrupt barriers using anaerobic reactions.

May be effective in slow groundwater flow conditions.
May be effective in slow groundwater flow conditions. 


\begin{tabular}{|c|c|c|c|c|}
\hline $\begin{array}{c}\text { Simple } \\
\text { Fast }\end{array}$ & $\begin{array}{c}\text { Simple } \\
\text { Slow }\end{array}$ & $\begin{array}{c}\text { Heterogeneous } \\
\text { Fast }\end{array}$ & $\begin{array}{c}\text { Heterogeneous } \\
\text { Slow }\end{array}$ & $\begin{array}{c}\text { Fractured/ } \\
\text { Porous Rock }\end{array}$ \\
\hline
\end{tabular}

\begin{tabular}{|l|l|l|}
\hline Aerobic & Anoxic & Anaerobic \\
\hline
\end{tabular}

\section{Key Source Control Concepts}

A description of potential source control measures, and their applicability to Scenario 4 sites, is shown below. Note that source control measures are unlikely to achieve complete restoration at a site, and some source material is always left behind after treatment (U.S. EPA, 2003; McGuire et al., 2006).

\begin{tabular}{|c|c|c|c|}
\hline Example Technology & $\begin{array}{l}\text { Performance } \\
\left(25^{\text {th }}-75^{\text {th }}\right. \\
\text { Percentile } \\
\% \text { reduction in } \\
\text { parent } \\
\text { compound) })^{1}\end{array}$ & $\begin{array}{l}\frac{\text { Unit Cost }}{25^{\text {th }}-75^{\text {th }}} \\
\text { Percentile } \\
(\$ / y r d)^{2}\end{array}$ & Applicability to Scenario 1 Sites \\
\hline In-situ biodegradation & $73-99$ & $27-152$ & $\begin{array}{l}\text { Well suited for anaerobic sites; enhances existing } \\
\text { biodegradation reactions. Suitable for most Scenario } 4 \text { sites. } \\
\text { Simple hydrogeology makes application easier. Slow } \\
\text { groundwater flow may make application easier than for high } \\
\text { flow rate conditions. Least expensive treatment option. }\end{array}$ \\
\hline Thermal treatment & $68-99.9$ & $48-129$ & Does not appear to disrupt MNA after treatment. \\
\hline Chemical oxidation & $70-97$ & $47-194$ & $\begin{array}{l}\text { Removes more total CVOCs than enhanced biodegradation } \\
\text { but shows more rebound }{ }^{1} \text {. Can change geochemistry of } \\
\text { Scenario } 4 \text { site to aerobic conditions for some period after } \\
\text { treatment. Can change microbial population and composition. } \\
\text { Simple hydrogeology makes application easier. May be more } \\
\text { suitable for anoxic or aerobic sites. }\end{array}$ \\
\hline Surfactant/cosolvents & $92-98$ & $118-1322$ & $\begin{array}{l}\text { High treatment efficiency but much higher cost. Costs reflect } \\
\text { some expensive pilot-scale projects. Simple hydrogeology } \\
\text { makes application easier. Some surfactants/cosolvents can } \\
\text { serve as electron donors for subsequent anaerobic } \\
\text { biodegradation reactions. }\end{array}$ \\
\hline Air sparging & - & - & $\begin{array}{l}\text { Not recommended at most sites. Addition of oxygen can } \\
\text { disrupt anaerobic processes. }\end{array}$ \\
\hline $\begin{array}{l}\text { Pump and Treat source } \\
\text { containment }\end{array}$ & NA & & $\begin{array}{l}\text { Due to the low groundwater flow rate, a relatively small } \\
\text { system may be sufficient. This approach does not reduce } \\
\text { mass significantly compared to the rate of mass loss without } \\
\text { P\&T and may need to be operated for a long time. }\end{array}$ \\
\hline
\end{tabular}




\begin{tabular}{|c|c|c|c|c|}
\hline $\begin{array}{c}\text { Simple } \\
\text { Fast }\end{array}$ & $\begin{array}{c}\text { Simple } \\
\text { Slow }\end{array}$ & $\begin{array}{c}\text { Heterogeneous } \\
\text { Fast }\end{array}$ & $\begin{array}{c}\text { Heterogeneous } \\
\text { Slow }\end{array}$ & $\begin{array}{c}\text { Fractured/ } \\
\text { Porous Rock }\end{array}$ \\
\hline
\end{tabular}

\begin{tabular}{|l|l|l|}
\hline Aerobic & Anoxic & Anaerobic \\
\hline
\end{tabular}

\section{SCENARIO NUMBER 5}

\section{Simple Slow Flow}

and

Anoxic
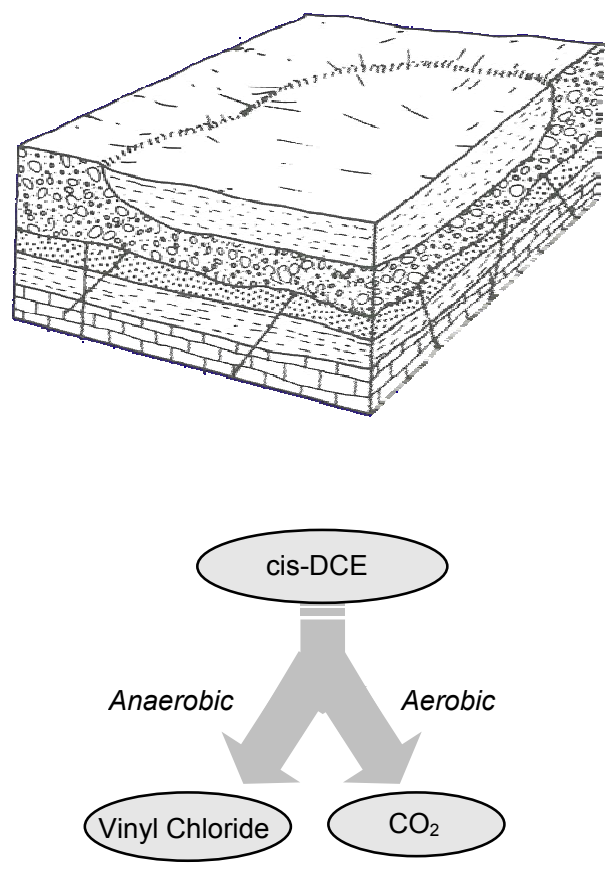


\begin{tabular}{|c|c|c|c|c|}
\hline $\begin{array}{c}\text { Simple } \\
\text { Fast }\end{array}$ & $\begin{array}{c}\text { Simple } \\
\text { Slow }\end{array}$ & $\begin{array}{c}\text { Heterogeneous } \\
\text { Fast }\end{array}$ & $\begin{array}{c}\text { Heterogeneous } \\
\text { Slow }\end{array}$ & $\begin{array}{c}\text { Fractured/ } \\
\text { Porous Rock }\end{array}$ \\
\hline
\end{tabular}

\begin{tabular}{|l|l|l|}
\hline \multicolumn{2}{|c|}{ GEOCHEMICAL SETTING } \\
\hline Aerobic & Anoxic & Anaerobic \\
& & \\
\hline
\end{tabular}

\section{SCENARIO 5 DESCRIPTION: SIMPLE SLOW FLOW and ANOXIC}

The hydrogeologic setting and geochemical setting below define the basic scenario type. This scenario has the following characteristics:

\section{Hydrogeologic Setting}

"Simple and Slow" Hydrogeology:

- Only one hydrogeologic unit

- Relatively uniform hydraulic conductivity

- Relatively low groundwater seepage velocity

(see Section 2.1 and Appendix 1 for more information)

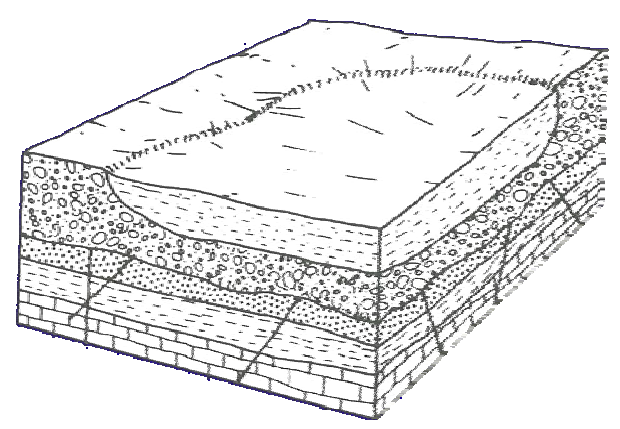

Block Diagram of Glacial Lake Deposit Aquifer from DRASTIC System

\section{Geochemical Setting}

"Anoxic" Geochemistry:

- Dissolved oxygen is low, redox is medium to low

- There are no, or limited, indicators of significant activity of anaerobic bacteria

(see Section 2.2 for more information)

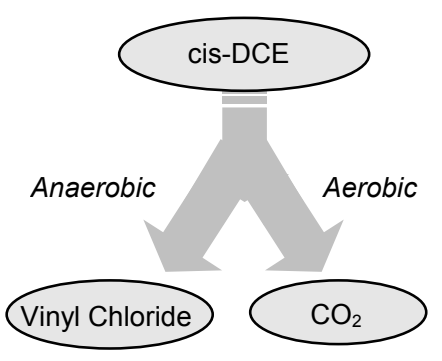

Example Reactions for "Anoxic" Geochemical Setting 


\begin{tabular}{|c|c|c|c|c|}
\hline $\begin{array}{c}\text { Simple } \\
\text { Fast }\end{array}$ & $\begin{array}{c}\text { Simple } \\
\text { Slow }\end{array}$ & $\begin{array}{c}\text { Heterogeneous } \\
\text { Fast }\end{array}$ & $\begin{array}{c}\text { Heterogeneous } \\
\text { Slow }\end{array}$ & $\begin{array}{c}\text { Fractured/ } \\
\text { Porous Rock }\end{array}$ \\
\hline
\end{tabular}

\begin{tabular}{|l|l|l|}
\hline Aerobic & Anoxic & Anaerobic \\
\hline
\end{tabular}

\section{KEY DECHLORINATION REACTIONS}

\section{Reaction Overview}

The chart to the right shows which reactions are likely to occur, which occur but at a slow rate, which may occur under specific conditions, and which are unlikely to occur.

\section{Typically Biodegradable Parent Compounds}

These compounds may be degradable under anoxic conditions:

- PCE

- TCE

- 1,1,1-TCA

- 1,2-DCA

- $\mathrm{CT}$

\section{Typical Daughter Products}

Daughter products that may be present depending on the parent compound and the reactions listed to the right:

- TCE

- $\quad$ cis 1,2-DCE

- VC

- 1,2-DCA

- 1,1-DCE

- $\mathrm{CF}$

See Section 5.1 for more information about reactions

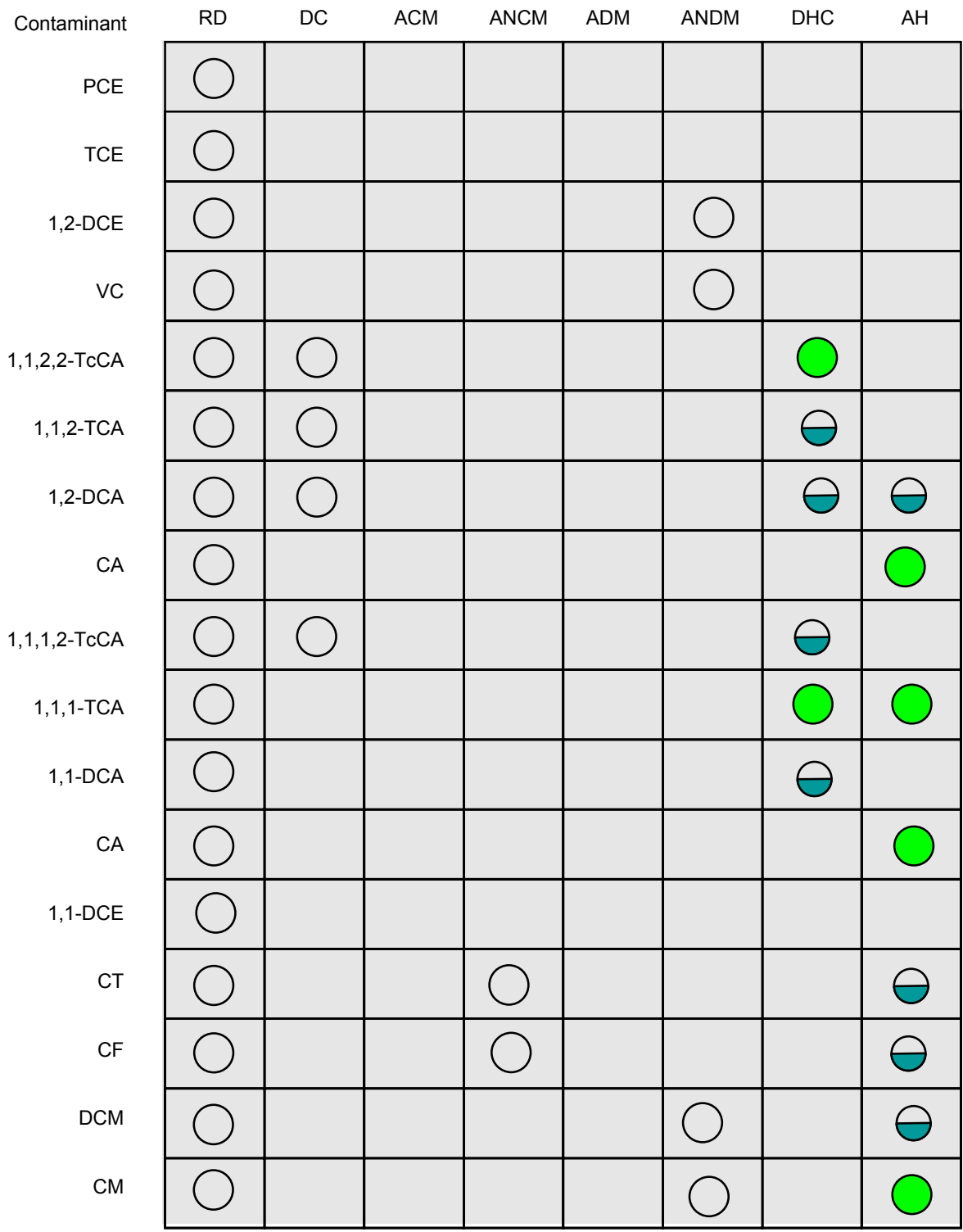

REACTIONS

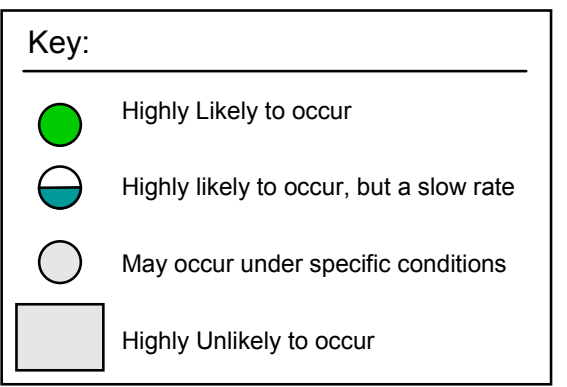

\begin{tabular}{|c|l|}
\hline ACM & Aerobic Co-Metabolism \\
\hline ANCM & Anaerobic Co-Metabolism \\
\hline ADM & Aerobic Direct Metabolism \\
\hline ANDM & Anaerobic Direct Metabolism \\
\hline DHC & Dehydrochlorination (abiotic) \\
\hline AH & Abiotic Hydrolysis \\
\hline DC & Dichloroelimination (biotic) \\
\hline RD & Reductive Dechlorination (hydrogenolysis) \\
\hline
\end{tabular}




\begin{tabular}{|c|c|c|c|c|}
\hline $\begin{array}{c}\text { Simple } \\
\text { Fast }\end{array}$ & $\begin{array}{c}\text { Simple } \\
\text { Slow }\end{array}$ & $\begin{array}{c}\text { Heterogeneous } \\
\text { Fast }\end{array}$ & $\begin{array}{c}\text { Heterogeneous } \\
\text { Slow }\end{array}$ & $\begin{array}{c}\text { Fractured/ } \\
\text { Porous Rock }\end{array}$ \\
\hline
\end{tabular}

\begin{tabular}{|l|l|l|}
\hline Aerobic & Anoxic & Anaerobic \\
\hline
\end{tabular}

\section{EFFECT OF MODIFYING FACTORS}

\begin{tabular}{|c|c|}
\hline MODIFYING FACTOR & KEY POINTS \\
\hline Source Strength & $\begin{array}{l}\text { IF YOU HAVE A STRONG SOURCE: } \\
\text { - Plumes (both parent and daughter compounds) may be longer } \\
\text { - } \quad \text { Source zones may persist for longer periods of time } \\
\text { - More likely to need EA or source control measures } \\
\text { IF YOU HAVE A MODERATE SOURCE: } \\
\text { - Intermediate condition between Strong and Weak Source } \\
\text { IF YOU HAVE A WEAK SOURCE: } \\
\text { - Plumes may be shorter } \\
\text { - } \quad \text { Source zones may not persist as long } \\
\text { - MNA alone or MNA with EA more likely to be sufficient }\end{array}$ \\
\hline Source & $\begin{array}{l}\text { IF YOU HAVE MOSTLY A VADOSE ZONE SOURCE: } \\
\text { - Source may appear small due to dilution but can be large } \\
\text { - Sources in clay vadose zones will be weaker but more long-lived than } \\
\text { sandy vadose zone } \\
\text { - Plumes will be thinner and closer to water table } \\
\text { IF YOU HAVE A SUBMERGED SOURCE: } \\
\text { - Simple, slow hydrogeology means matrix diffusion may be important, but } \\
\text { - less important than at complex sites. } \\
\text { - Source mass flux can decrease relatively rapidly as DNAPL fingers } \\
\text { IF YOU HAVE A MIXED SOURCE: } \\
\text { - May have combination of the factors above }\end{array}$ \\
\hline Travel Tim & $\begin{array}{l}\text { CLOSE RECEPTORS (<2 YEARS TRAVEL TIME) } \\
\text { - More intensive monitoring system likely to be needed due to potential } \\
\text { serious consequences in event of failure of MNA/EA. } \\
\text { MODERATE RECEPTORS ( }>2 \text { but < } 5 \text { YEARS TRAVEL TIME) } \\
\text { - Represents middle-ground case } \\
\text { DISTANT RECEPTORS (>5 YEARS TRAVEL TIME) } \\
\text { - Less intensive monitoring system likely to be needed due to lower } \\
\text { potential for serious consequences in event of failure of MNA/EA. } \\
\text { MNA alone or MNA with EA more likely to be sufficient }\end{array}$ \\
\hline Plume Stability & $\begin{array}{l}\text { EXPANDING OR PERTURBED }{ }^{1} \text { PLUME } \\
\text { - More intensive monitoring system likely to be needed } \\
\text { STABLE PLUME } \\
\text { SHRINKING PLUME } \\
\text { - Represents middle-ground case } \\
\text { - } \quad \text { MNA alone likely to be sufficient } \\
{ }^{1} \text { For instance if the plume has been impacted by a previous remedy such as P\&T. }\end{array}$ \\
\hline
\end{tabular}




\begin{tabular}{|c|c|c|c|c|c|c|c|}
\hline $\begin{array}{l}\text { Simple } \\
\text { Fast }\end{array}$ & $\begin{array}{l}\text { Simple } \\
\text { Slow }\end{array}$ & $\begin{array}{c}\text { Heterogeneous } \\
\text { Fast }\end{array}$ & $\begin{array}{c}\text { Heterogeneous } \\
\text { Slow }\end{array}$ & $\begin{array}{l}\text { Fractured/ } \\
\text { Porous Rock }\end{array}$ & Aerobic & Anoxic & Anaerobic \\
\hline
\end{tabular}

\section{WILL MNA WORK?}

\section{Potential for MNA Processes to Control Plume}

Because at Scenario 5 sites there are not clear indicators that the type of conditions conducive to MNA are present, it is initially uncertain whether natural attenuation processes will be suitable to manage the contaminants in the plume or plume segment. Typically, more in-depth investigation of the site attenuation processes and more rigorous monitoring are needed to evaluate the extent of natural attenuation processes and the ability of MNA to meet the remediation objectives. Some form of enhanced attenuation may be needed to couple with MNA as the remedy.

In a slow-flowing heterogeneous aquifer, relatively low rates of contaminant degradation can stabilize the plume. If reductive dechlorination processes are occurring under these conditions, it would be expected that the daughter product plume would be larger than the parent product plume because the degradation rate of daughters is typically slower than the rate for the parents.

If the plume is shown to be either stable or shrinking then natural attenuation processes (primarily reductive dechlorination) alone have been vigorous enough to date to prevent further migration of the plume or plume segment. Under these conditions MNA may be appropriate, but it may still be difficult to identify the specific attenuation mechanism under the anoxic geochemical conditions.

At some Scenario 5 sites, "DCE stall" may be of concern and an indication that conditions are not suitable for complete dechlorination of the source contaminants. DCE stall is an informal term typically used to describe conditions at chlorinated ethene sites where the cis-1,2-DCE "stalls outs" or exhibits a very low conversion rate to VC. This DCE "stall" condition has been ascribed to a variety of factors, including:

- Lack of the necessary microbiological communities that are required to degrade cis-1,2-DCE to VC;

- The direct conversion of cis-1,2-DCE to carbon dioxide, which makes it appear that cis-1,2-DCE is not being biodegraded because VC is not being produced; but in fact the cis-1,2-DCE is being biodegraded by direct oxidation to carbon dioxide;

- Conditions which are anaerobic enough to support the conversion of TCE to cis-1,2-DCE but not anaerobic enough to support the conversion from cis-1,2-DCE to VC by reductive dechlorination;

- Toxicity effects caused at sites where sulfate reducers are producing hydrogen sulfide $\left(\mathrm{H}_{2} \mathrm{~S}\right)$, but the $\mathrm{H}_{2} \mathrm{~S}$ is not being precipitated fast enough by ferrous iron (a by-product of ferric iron reduction) to prevent toxicity effects in the cis-1,2-DCE degraders.

While the cause of cis-1,2-DCE stall is still being evaluated by a number of researchers, the main implication is that at some chlorinated ethene sites, cis-1,2-DCE plumes are expanding and not being controlled. DCE "stall" does not affect long-term sustainability of a reaction, but does determine if natural attenuation processes are sufficient to prevent migration of the plume.

\section{Key Sustainability Concept}

Slow-flowing plume segments may have only low or moderate mass flux of contaminants leaving the source, and therefore moderate rates of degradation are often sufficient to attenuate the plume. Sufficient natural substrate (e.g., organic matter) or co-contaminants that serve as electron donors and can act as a substrate are needed to sustain any anaerobic reactions using the chlorinated solvent as an electron acceptor. For some contaminants under anoxic conditions, biological reactions use the chlorinated solvent as the electron donor. At Scenario 5 sites, nonbiologically catalyzed attenuation processes may be the primary attenuation processes. In this case, the processes are likely sustainable, but may be difficult to identify and quantify. 


\begin{tabular}{|c|c|c|c|c|c|c|c|}
\hline $\begin{array}{l}\text { Simple } \\
\text { Fast }\end{array}$ & $\begin{array}{l}\text { Simple } \\
\text { Slow }\end{array}$ & $\begin{array}{c}\text { Heterogeneous } \\
\text { Fast }\end{array}$ & $\begin{array}{c}\text { Heterogeneous } \\
\text { Slow }\end{array}$ & $\begin{array}{l}\text { Fractured/ } \\
\text { Porous Rock }\end{array}$ & Aerobic & Anoxic & Anaerobic \\
\hline
\end{tabular}

\section{HOW DO I CHARACTERIZE THIS TYPE OF SITE?}

\section{Actions Needed to Determine MNA Viability}

The following are key considerations for evaluating the viability of MNA at a Simple Slow Flow and Anoxic type site:

- $\quad$ assess the site geochemical, hydraulic, and contaminant conditions in detail to assess the type and extent/rate of attenuation processes - this assessment may require significant effort depending on the site conditions, however, the uniform hydraulic conditions will help simplify some parts of the assessment;

- $\quad$ both parent compound and daughter compounds need to be delineated (the anoxic setting means that a number of daughter products may be generated);

- $\quad$ determine if plume(s) are expanding/perturbed, stable, or shrinking;

- determine the location of any receptors (if present) and determine the travel time from the edge of the plume to these receptors;

- a mass-balance type evaluation of the source zone will help to determine if the electron donor supply is sustainable over the long term if the attenuation reactions are determined to be primarily anaerobic dechlorination with the contaminant acting as the electron acceptor.

\section{Key Monitoring Concepts}

You will likely need a set of transect wells along the plume centerline and some sentry wells - looks like the "text book" case due to the simple plume shape in a homogenous aquifer. However, if characterization indicates that there is spatial variability in the geochemical conditions, monitoring for the specific geochemical areas may increase the number of wells needed compared to sites with more uniform geochemical conditions.

The slow hydrogeologic setting may mean the plume is of moderate size. However, a long period of time may be required for the plume to become stable (if it is going to). Thus a long temporal record (i.e., the number of years of monitoring data you have) may be needed to determine plume stability.

\section{Key Uncertainty Concepts}

The anoxic geochemical setting may cause considerable uncertainty in evaluating MNA because it may be difficult to identify and quantify the attenuation processes. There may be uncertainty about the plume conditions in general under this scenario because the plume may not be fully developed due to the slow flow conditions and it may not be evident if MNA is currently working. 


\begin{tabular}{|c|c|c|c|c|}
\hline $\begin{array}{c}\text { Simple } \\
\text { Fast }\end{array}$ & $\begin{array}{c}\text { Simple } \\
\text { Slow }\end{array}$ & $\begin{array}{c}\text { Heterogeneous } \\
\text { Fast }\end{array}$ & $\begin{array}{c}\text { Heterogeneous } \\
\text { Slow }\end{array}$ & $\begin{array}{c}\text { Fractured/ } \\
\text { Porous Rock }\end{array}$ \\
\hline
\end{tabular}

\begin{tabular}{|l|l|l|}
\hline \multicolumn{3}{|c|}{ GEOCHEMICAL SETTING } \\
\hline Aerobic & Anoxic & Anaerobic \\
\hline
\end{tabular}

\section{HOW DO I ANALYZE DATA?}

In a slow-flowing aquifer, a plume is more likely to show concentration differences over short distances and the plume will become stable (if it is going to) over a long period of time. Thus, while a good first step in this type of aquifer is to examine plume maps, concentration vs. time at each well, and concentration vs. distance plots to determine whether the plume is attenuating, additional information may be needed. A simple transport model such as BIOCHLOR can be helpful as a screening tool in analyzing and visualizing the data and expected plume conditions for comparison to field data. However, BIOCHLOR will not be sufficient to describe the range of attenuation processes that may be important under anoxic geochemical conditions. As such, more complex numerical modeling is more likely to be needed as part of MNA evaluation for Scenario 5 sites. To support this more complex analysis, microcosm tests, molecular probes, and more detailed field measurements may be required. The chart below summarizes an approach for analyzing data at sites depending on whether the concentration data indicates that the plume is decreasing, stable, or increasing and the source type. As noted in the table, as the source gets stronger and the plume is less likely to be decreasing in extent, more information is needed to support selection of an MNA remedy.

\begin{tabular}{|c|c|c|c|}
\hline & \multicolumn{3}{|c|}{ PLUME STATUS } \\
\hline $\begin{array}{l}\text { CONTAMINANT } \\
\text { CONCENTRATIONS/GEOCHEMICAL } \\
\text { STATUS }\end{array}$ & $\begin{array}{l}\text { DECREASING OR } \\
\text { PROBABLY } \\
\text { DECREASING }\end{array}$ & STABLE & $\begin{array}{l}\text { INCREASING, } \\
\text { PROBABLY } \\
\text { INCREASING, OR } \\
\text { PERTURBED }^{1}\end{array}$ \\
\hline Weak Source & - Mass loss & $\begin{array}{ll}\text { - } & \text { Mass loss } \\
\text { - } & \text { Geochemical footprints } \\
\text { - } & \text { Simple Model/Special } \\
& \text { Studies }\end{array}$ & $\begin{array}{l}\text { - } \text { Mass loss } \\
\text { - } \text { Geochemical footprints } \\
\text { - } \text { Comprehensive Model/ } \\
\text { Special Studies }\end{array}$ \\
\hline Strong Source & $\begin{array}{l}\text { - Mass loss } \\
\text { - Geochemical footprints } \\
\text { - Simple Model }\end{array}$ & $\begin{array}{ll}\text { - } & \text { Mass loss } \\
\text { - } & \text { Geochemical footprints } \\
\text { - } & \text { Comprehensive Model/ } \\
\text { Special Studies }\end{array}$ & $\begin{array}{ll}\text { - } & \text { Mass loss } \\
\text { - } & \text { Geochemical footprints } \\
\text { - } & \text { Comprehensive Model/ } \\
\text { Special Studies }\end{array}$ \\
\hline
\end{tabular}

${ }^{1}$ For instance if the plume has been impacted by a previous remedy such as P\&T.

To demonstrate mass loss construct these graphics:

- Concentration vs. time plots at individual wells;

- Concentration vs. distance plots, with multiple lines for different sampling events through time;

- Plume maps showing plume extent at different times (i.e., either panel maps, or one map with several plume boundaries for different times).

To show geochemical footprints make tables or figures that show:

- Daughter product production;

- Ethene/Ethane production;

- Chloride production (this may not work at many sites, however, due to background chloride);

- Low dissolved oxygen (shows anoxic geochemical conditions);

- Methane and iron(II) distribution (indicators of anaerobic activity);

- Nitrate and sulfate distribution (indicators of competing electron acceptors);

To perform modeling, typical tools include the following:

- Simple transport model (analytical model, e.g., BIOCHLOR, BIOBALANCE ${ }^{1}$ );

- Comprehensive transport model (numerical model, e.g., RT3D).

If a special study is needed, some of the following may be applicable:

- Carbon/chlorine isotope analysis (indicator of degradation processes)

- Molecular probes (indicators of microbial activity when other geochemical indicators are ambiguous);

- Microcosm tests (determine the reaction processes occurring at the site).

${ }^{1}$ Developed by Groundwater Services (www.gsi-net.com), with the support of the Savannah River National Laboratory and $\mathrm{DOE}$, to evaluate monitored natural attenuation at chlorinated solvent sites. 


\begin{tabular}{|c|c|c|c|c|}
\hline $\begin{array}{c}\text { Simple } \\
\text { Fast }\end{array}$ & $\begin{array}{c}\text { Simple } \\
\text { Slow }\end{array}$ & $\begin{array}{c}\text { Heterogeneous } \\
\text { Fast }\end{array}$ & $\begin{array}{c}\text { Heterogeneous } \\
\text { Slow }\end{array}$ & $\begin{array}{c}\text { Fractured/ } \\
\text { Porous Rock }\end{array}$ \\
\hline
\end{tabular}

\begin{tabular}{|l|c|c|}
\hline \multicolumn{2}{|c|}{ GEOCHEMICAL SETTING } \\
\hline Aerobic & Anoxic & Anaerobic \\
\hline
\end{tabular}

\section{WHAT ABOUT COSTS AND ENHANCEMENTS?}

Costs for evaluating and implementing MNA for this scenario are primarily dependent on the following items.

- $\quad$ Source strength - Stronger and longer lasting sources will be more costly.

- $\quad$ Depth to the plume and size of the plume - Deeper, larger plumes require more and costlier monitoring wells.

- $\quad$ Travel time to the receptor - Plumes closer to receptors will require more frequent monitoring and over a long period of time with the slow groundwater flow rate.

- $\quad$ Plume stability - Less stable plumes require more rigorous evaluation and monitoring to select and implement MNA. With the slow groundwater flow rate, more rigorous evaluation or a longer period of monitoring may cause higher costs than for sites with a high groundwater flow rate.

- Extent of variability in geochemical conditions - More variability will likely require more characterization and monitoring to assess attenuation conditions within each different geochemical zone.

MNA may be a viable single remedy for the site. If it is determined that MNA may not meet remediation goals, a good first option is to evaluate the potential use of sustainable enhancements (enhanced attenuation). The objective being to adjust the attenuation conditions sufficiently such that the plume is controlled. If the enhancements are insufficient to control the plume, source control treatment may be required. In general, enhanced attenuation is less likely a viable option for a fast flow regime with a strong source, unless source treatment is undertaken initially. The sections below discuss options for enhanced attenuation and source control related to this scenario.

\section{Key Enhanced Attenuation Concepts}

Enhancements are presented organized by the different zones in which they may be applied: source zone (reduction of contaminant mass flux to plume); plume (enhanced attenuation processes); or discharge zone (enhanced attenuation processes). Within the source zone enhancements can be applied as a hydraulic manipulation or as a passive source reduction (active source control is discussed in the next section). Within the plume and discharge zone, either biological (microbial or plant based) or abiotic (abiotic degradation, reactive barriers, sorption) attenuation processes can be enhanced. A description of potential enhanced attenuation approaches, and their applicability to Scenario 5 sites, is shown below. More detailed information about each technology listed below is available in Early et al., (2005). 


\begin{tabular}{|c|c|c|c|c|}
\hline $\begin{array}{c}\text { Simple } \\
\text { Fast }\end{array}$ & $\begin{array}{c}\text { Simple } \\
\text { Slow }\end{array}$ & $\begin{array}{c}\text { Heterogeneous } \\
\text { Fast }\end{array}$ & $\begin{array}{c}\text { Heterogeneous } \\
\text { Slow }\end{array}$ & $\begin{array}{c}\text { Fractured/ } \\
\text { Porous Rock }\end{array}$ \\
\hline
\end{tabular}

\begin{tabular}{|l|c|c|}
\hline \multicolumn{3}{|c|}{ GEOCHEMICAL SETTING } \\
\hline Aerobic & Anoxic & Anaerobic \\
\hline
\end{tabular}

\section{Enhancement Summary}

\begin{tabular}{l}
\multicolumn{1}{c}{ Enhancement } \\
\hline SOURCE ZONE ENHANCE \\
Surface water or \\
groundwater \\
interception/diversion
\end{tabular}

Physical containment

Passive extraction

Enhanced

biodegradation

Permeable reactive barrier

\section{Description}

Use of interception trenches or wells, surface covers, or phyto-covers (plants) to reduce water flux through source area and/or divert unwanted electron acceptors.

Use of grout walls and other physical containment

Use of passive soil vapor extraction

Injection of electron donor (e.g., HRC, molasses, vegetable oil) to enhance microbial degradation of the source.

Use of zero valent iron, reduced sediment iron, enhanced partitioning, or biological barrier to attenuate some of the contaminant flux at the downgradient edge of the source area.

\section{PLUME AND DISCHARGE ZONE ENHANCEMENTS}

Enhanced
biodegradation

Permeable reactive barrier

Phytoextraction

Plant-based hydraulic control (plume enhancement only)
Injection of electron donor (e.g., HRC, molasses, vegetable oil) to enhance microbial degradation of the source.

Use of zero valent iron, reduced sediment iron, enhanced partitioning, or biological barrier to attenuate some of the contaminant flux within the plume.

Use of plants to extract contaminants from near surface groundwater

Use of plants to control hydraulic gradient and slow groundwater
Likely more applicable using surface covers or phytocovers if the source is primarily within the vadose zone. With slow flow rate, this method may be relatively effective compared to application in higher flow rate conditions.

Potentially applicable depending on the geometry of the source zone.

\section{Useful if the source is primarily within the vadose} zone.

Potentially well suited for anoxic sites because oxygen concentrations are already low and anaerobic processes may be readily stimulated. Simple hydrogeology makes application easier. Slow groundwater flow may make application easier than for high flow rate conditions.

Easier to apply in slow flow regime. Slower groundwater flows potentially result in less contaminant and competing electron acceptors passing through the barrier, requiring less thickness to achieve desired treatment levels. Anoxic conditions are helpful because oxygen concentration is low and will not disrupt barriers using anaerobic reactions.

Potentially well suited for anoxic sites because oxygen concentrations are already low and anaerobic processes may be readily stimulated. Simple hydrogeology makes application easier. Slow groundwater flow may make application easier than for high flow rate conditions.

Easier to apply in slow flow regime. Slower groundwater flows potentially result in less contaminant and competing electron acceptors passing through the barrier, requiring less thickness to achieve desired treatment levels. Anoxic conditions are helpful because oxygen concentration is low and will not disrupt barriers using anaerobic reactions.

\section{May be effective in slow groundwater flow conditions.}

May be effective in slow groundwater flow conditions. 


\begin{tabular}{|c|c|c|c|c|}
\hline $\begin{array}{c}\text { Simple } \\
\text { Fast }\end{array}$ & $\begin{array}{c}\text { Simple } \\
\text { Slow }\end{array}$ & $\begin{array}{c}\text { Heterogeneous } \\
\text { Fast }\end{array}$ & $\begin{array}{c}\text { Heterogeneous } \\
\text { Slow }\end{array}$ & $\begin{array}{c}\text { Fractured/ } \\
\text { Porous Rock }\end{array}$ \\
\hline
\end{tabular}

\begin{tabular}{|l|c|c|}
\hline \multicolumn{3}{|c|}{ GEOCHEMICAL SETTING } \\
\hline Aerobic & Anoxic & Anaerobic \\
\hline
\end{tabular}

\section{Key Source Control Concepts}

A description of potential source control measures, and their applicability to Scenario 5 sites, is shown below. Note that source control measures are unlikely to achieve complete restoration at a site, and some source material is always left behind after treatment (U.S. EPA, 2003; McGuire et al., 2006).

\begin{tabular}{|c|c|c|c|}
\hline Example Technology & $\begin{array}{l}\text { Performance } \\
\left(25^{\text {th }}-75^{\text {th }}\right. \\
\text { Percentile } \\
\% \text { reduction in } \\
\text { parent } \\
\text { compound })^{1}\end{array}$ & $\begin{array}{l}\text { Unit Cost } \\
25^{\text {th }}-75^{\text {th }} \\
\text { Percentile } \\
(\$ / y r d)^{2}\end{array}$ & Applicability to Scenario 5 Sites \\
\hline In-situ biodegradation & $73-99$ & $27-152$ & $\begin{array}{l}\text { Potentially well suited for anoxic sites because oxygen } \\
\text { concentrations are already low and anaerobic processes may be } \\
\text { readily stimulated. Simple hydrogeology makes application } \\
\text { easier. Slow groundwater flow may make application easier } \\
\text { than for high flow rate conditions. Least expensive treatment } \\
\text { option. }\end{array}$ \\
\hline Thermal treatment & $68-99.9$ & $48-129$ & Does not appear to disrupt MNA after treatment. \\
\hline Chemical oxidation & $70-97$ & $47-194$ & $\begin{array}{l}\text { Removes more total CVOCs than enhanced biodegradation but } \\
\text { shows more rebound }{ }^{1} \text {. Can change geochemistry of Scenario } 5 \\
\text { site to aerobic conditions for some period after treatment. Can } \\
\text { change microbial population and composition. Simple } \\
\text { hydrogeology makes application easier. }\end{array}$ \\
\hline Surfactant/cosolvents & $92-98$ & $118-1322$ & $\begin{array}{l}\text { High treatment efficiency but much higher cost. Costs reflect } \\
\text { some expensive pilot-scale projects. Simple hydrogeology } \\
\text { makes application easier. Some surfactants/cosolvents can } \\
\text { serve as electron donors for subsequent anaerobic } \\
\text { biodegradation reactions. }\end{array}$ \\
\hline Air sparging & - & - & $\begin{array}{l}\text { May be suitable if only limited biological attenuation is occurring } \\
\text { at a site. Addition of oxygen can disrupt anaerobic processes } \\
\text { that may be occurring. }\end{array}$ \\
\hline $\begin{array}{l}\text { Pump and Treat source } \\
\text { containment }\end{array}$ & NA & & $\begin{array}{l}\text { Due to the low groundwater flow rate, a relatively small system } \\
\text { may be sufficient. This approach does not reduce mass } \\
\text { significantly compared to the rate of mass loss without P\&T and } \\
\text { may need to be operated for a long time. }\end{array}$ \\
\hline
\end{tabular}




\begin{tabular}{|c|c|c|c|c|}
\hline $\begin{array}{c}\text { Simple } \\
\text { Fast }\end{array}$ & $\begin{array}{c}\text { Simple } \\
\text { Slow }\end{array}$ & $\begin{array}{c}\text { Heterogeneous } \\
\text { Fast }\end{array}$ & $\begin{array}{c}\text { Heterogeneous } \\
\text { Slow }\end{array}$ & $\begin{array}{c}\text { Fractured/ } \\
\text { Porous Rock }\end{array}$ \\
\hline
\end{tabular}

\section{SCENARIO NUMBER 6}

\section{Simple Slow Flow \\ and}

\section{Aerobic}

\section{Draft}
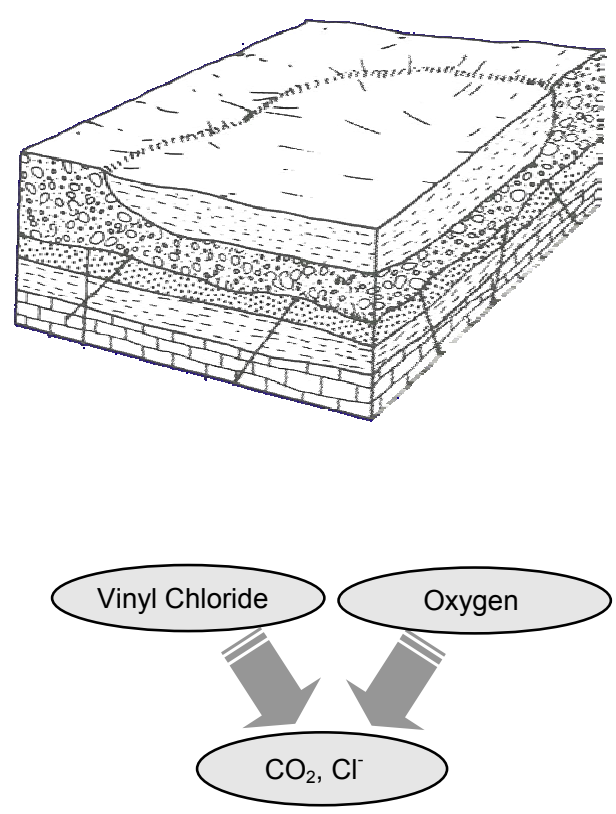


\begin{tabular}{|c|c|c|c|c|}
\hline $\begin{array}{c}\text { Simple } \\
\text { Fast }\end{array}$ & $\begin{array}{c}\text { Simple } \\
\text { Slow }\end{array}$ & $\begin{array}{c}\text { Heterogeneous } \\
\text { Fast }\end{array}$ & $\begin{array}{c}\text { Heterogeneous } \\
\text { Slow }\end{array}$ & $\begin{array}{c}\text { Fractured/ } \\
\text { Porous Rock }\end{array}$ \\
\hline
\end{tabular}

\begin{tabular}{|l|c|c|}
\hline \multicolumn{3}{|c}{ GEOCHEMICAL SETTING } \\
\hline Aerobic & Anoxic & Anaerobic \\
\hline
\end{tabular}

\section{SCENARIO 6 DESCRIPTION: SIMPLE SLOW FLOW and AEROBIC}

The hydrogeologic setting and geochemical setting below define the basic scenario type. This scenario has the following characteristics:

\section{Hydrogeologic Setting}

"Simple and Slow" Hydrogeology:

- Only one hydrogeologic unit

- Relatively uniform hydraulic conductivity

- Relatively low groundwater seepage velocity

(see Section 2.1 and Appendix 1 for more information)

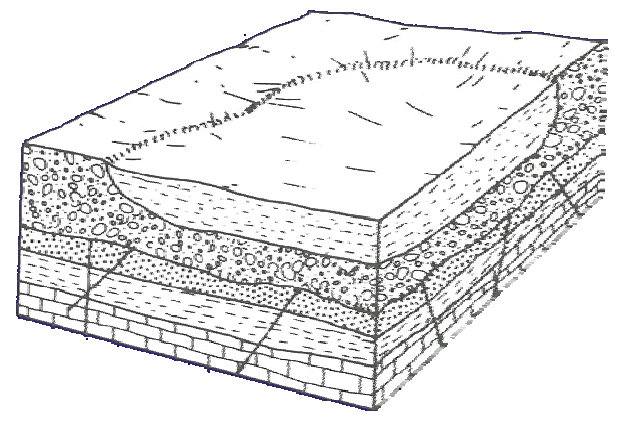

Block Diagram of Glacial Lake Deposit Aquifer from DRASTIC System

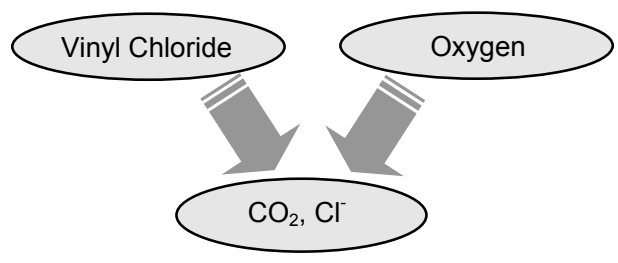

Example Reactions for "Aerobic" Geochemical Setting 


\begin{tabular}{|c|c|c|c|c|}
\hline $\begin{array}{c}\text { Simple } \\
\text { Fast }\end{array}$ & $\begin{array}{c}\text { Simple } \\
\text { Slow }\end{array}$ & $\begin{array}{c}\text { Heterogeneous } \\
\text { Fast }\end{array}$ & $\begin{array}{c}\text { Heterogeneous } \\
\text { Slow }\end{array}$ & $\begin{array}{c}\text { Fractured/ } \\
\text { Porous Rock }\end{array}$ \\
\hline
\end{tabular}

\section{KEY DECHLORINATION REACTIONS}

\section{Reaction Overview}

The chart to the right shows which reactions are likely to occur, which occur but at a slow rate, and which may occur under specific conditions, and which are unlikely to occur.

\section{Compounds Easier for Biological Degradation}

- $\quad$ cis 1,2-DCE

- $\mathrm{VC}$

- 1,2-DCA

- 1,1-DCE

- DCM

- $\mathrm{CM}$

\section{Compounds More Difficult 1 Biological Degradation}

- PCE

- TCE

- $\mathrm{CT}$

- $\mathrm{CF}$

See Section 5.1 for more information about reactions

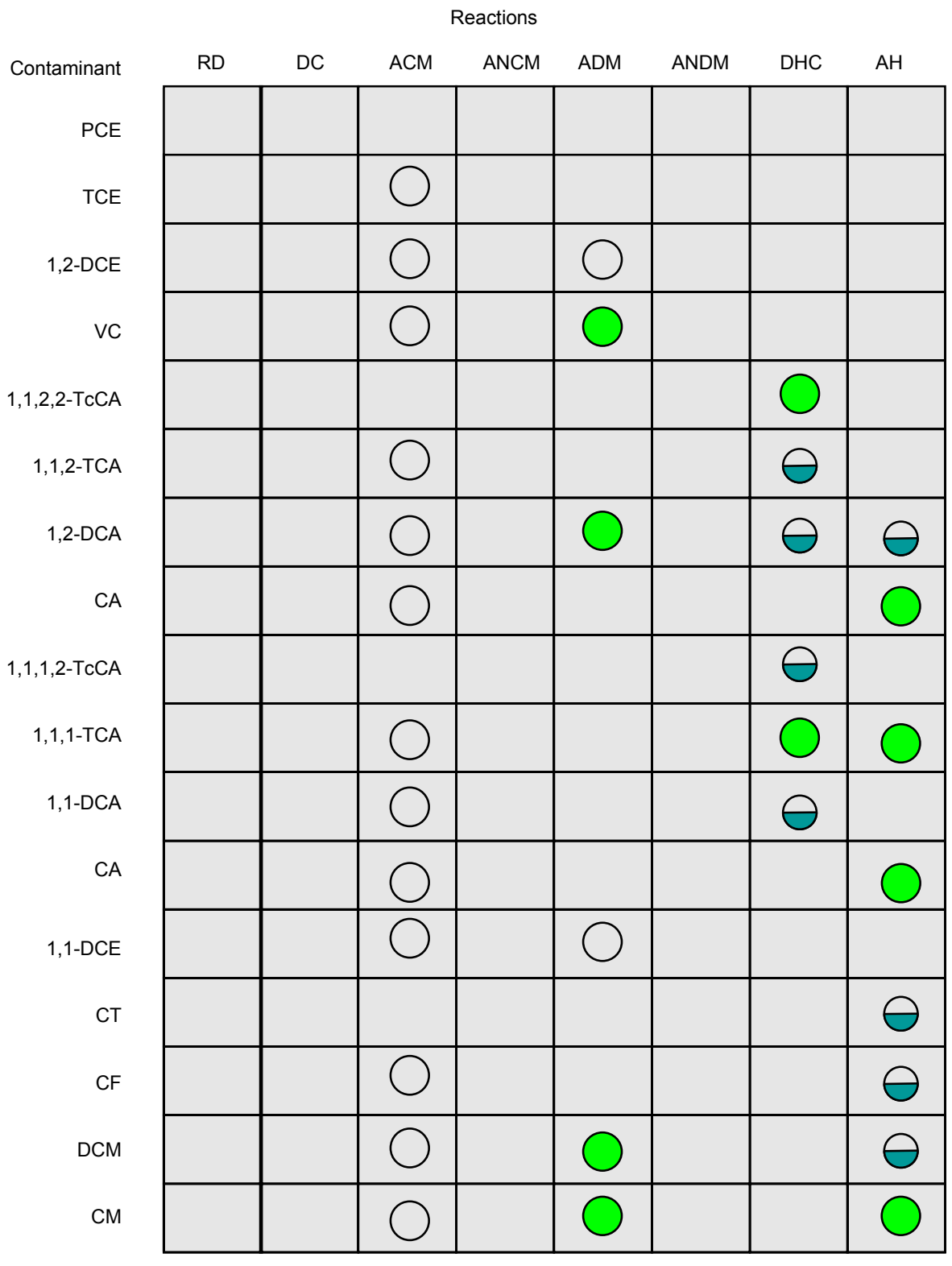

REACTIONS

\begin{tabular}{ll} 
Key: & \\
\hline & Highly Likely to occur \\
& Highly likely to occur, but a slow rate \\
& Highly occur under specific conditions
\end{tabular}




\begin{tabular}{|c|c|c|c|c|}
\hline $\begin{array}{c}\text { Simple } \\
\text { Fast }\end{array}$ & $\begin{array}{c}\text { Simple } \\
\text { Slow }\end{array}$ & $\begin{array}{c}\text { Heterogeneous } \\
\text { Fast }\end{array}$ & $\begin{array}{c}\text { Heterogeneous } \\
\text { Slow }\end{array}$ & $\begin{array}{c}\text { Fractured/ } \\
\text { Porous Rock }\end{array}$ \\
\hline
\end{tabular}

\section{EFFECT OF MODIFYING FACTORS}

\begin{tabular}{|c|c|}
\hline MODIFYING FACTOR & KEY POINTS \\
\hline Source Stre & $\begin{array}{l}\text { IF YOU HAVE A STRONG SOURCE: } \\
\text { - Plumes (both parent and daughter compounds) may be longer } \\
\text { - } \quad \text { Source zones may persist for longer periods of time } \\
\text { - More likely to need EA or source control measures } \\
\text { IF YOU HAVE A MODERATE SOURCE: } \\
\text { - Intermediate condition between Strong and Weak Source } \\
\text { IF YOU HAVE A WEAK SOURCE: } \\
\text { - Plumes may be shorter } \\
\text { - } \quad \text { Source zones may not persist as long } \\
\text { - MNA alone or MNA with EA more likely to be sufficient }\end{array}$ \\
\hline Source & 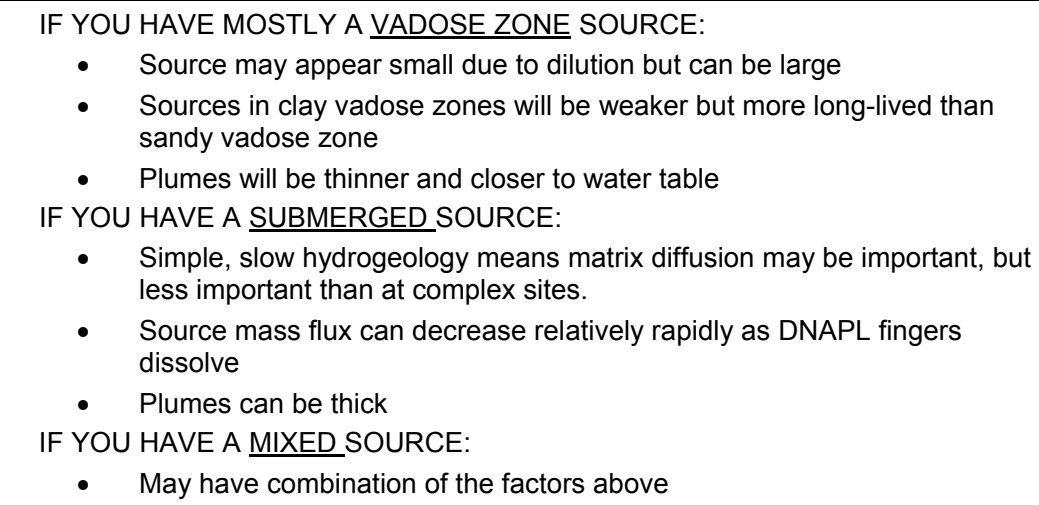 \\
\hline Travel Time to Receptors & $\begin{array}{l}\text { CLOSE RECEPTORS (<2 YEARS TRAVEL TIME) } \\
\text { - More intensive monitoring system likely to be needed due to potential } \\
\text { - } \\
\text { Morious consequences in event of failure of MNA/EA. } \\
\text { MODERATE RECEPTORS (>2 but < } 5 \text { YEARS TRAVEL TIME) } \\
\text { - Represents middle-ground case } \\
\text { DISTANT RECEPTORS ( }>5 \text { YEARS TRAVEL TIME) } \\
\text { - Less intensive monitoring system likely to be needed due to lower } \\
\text { - } \text { Motential for serious consequences in event of failure of MNA/EA. }\end{array}$ \\
\hline Plume Stability & $\begin{array}{l}\text { EXPANDING OR PERTURBED }{ }^{1} \text { PLUME } \\
\text { - More intensive monitoring system likely to be needed } \\
\text { STABLE PLUME } \\
\text { - Represents middle-ground case } \\
\text { SHRINKING PLUME } \\
\text { - Less intensive monitoring system likely to be needed } \\
{ }^{1} \text { For instance if the plume has been impacted by a previous remedy such as P\&T. }\end{array}$ \\
\hline
\end{tabular}




\begin{tabular}{|c|c|c|c|c|c|c|c|}
\hline $\begin{array}{c}\text { Simple } \\
\text { Fast }\end{array}$ & $\begin{array}{c}\text { Simple } \\
\text { Slow }\end{array}$ & $\begin{array}{c}\text { Heterogeneous } \\
\text { Fast }\end{array}$ & $\begin{array}{c}\text { Heterogeneous } \\
\text { Slow }\end{array}$ & $\begin{array}{c}\text { Fractured/ } \\
\text { Porous Rock }\end{array}$ \\
\hline
\end{tabular}

\section{WILL MNA WORK?}

\section{Potential for MNA Processes to Control Plume}

The slow hydrogeology means that plumes are less likely to be long. In slow-flowing homogeneous plumes or plume segments where aerobic conditions are present uniformly throughout the plume, there is less likelihood that natural attenuation processes will result in stable or shrinking plumes than in anaerobic plumes or plume segments if parent compounds such as PCE and TCE are present. While TCE and some other parent compounds can be degraded biologically under aerobic conditions, these reactions are co-metabolic reactions that require the presence of methane or another similar substrate that are typically not present in aerobic aquifers under natural conditions. Abiotic degradation processes will occur for some compounds, but may produce daughter products that cannot be readily degraded under aerobic conditions. Some compounds can be degraded directly by aerobic bacteria (e.g., DCE and VC). In summary, aerobic conditions are generally less conducive for managing chlorinated solvent plumes, except for a plume segment downgradient of an anaerobic plume segment where the contamination is dominated by reductive dechlorination daughter products such as cis-1,2-DCE or VC that can be directly degraded under aerobic conditions.

The slow nature of the hydrogeologic setting means that: i) there will be a low mass flux of oxygen entering the plume segment, so direct biodegradation reactions may be oxygen-limited; and ii) plumes of aerobically degradable contaminants may be relatively short.

\section{Key Sustainability Concept}

Direct aerobic biologic reactions and abiotic reactions are likely to be sustainable indefinitely unless the mass flux of oxygen is insufficient to support the degradation reactions.

Other biodegradation reactions that can occur under aerobic conditions are co-metabolic reactions that require oxygen and a primary substrate (such as methane). The probability that the supply of dissolved oxygen to the plume from upgradient sources (and plume re-aeration to a lesser degree) will be interrupted is relatively low. However, changes in source structure over time could result in reduced delivery of the primary substrate, increasing the uncertainty in the long-term sustainability of a naturally occurring co-metabolic reaction. 


\begin{tabular}{|c|c|c|c|c|c|c|c|}
\hline $\begin{array}{c}\text { Simple } \\
\text { Fast }\end{array}$ & $\begin{array}{c}\text { Simple } \\
\text { Slow }\end{array}$ & $\begin{array}{c}\text { Heterogeneous } \\
\text { Fast }\end{array}$ & $\begin{array}{c}\text { Heterogeneous } \\
\text { Slow }\end{array}$ & $\begin{array}{c}\text { Fractured/ } \\
\text { Porous Rock }\end{array}$ \\
\hline
\end{tabular}

\section{HOW DO I CHARACTERIZE THIS TYPE OF SITE?}

\section{Actions Needed to Determine MNA Viability:}

The following are key considerations for evaluating the viability of MNA at a Simple Slow Flow and Aerobic type site:

- $\quad$ trends for contaminant concentrations need to be established to assess whether attenuation is occurring (the aerobic setting means that a daughter products will likely not be available to assess whether attenuation processes are occurring);

- confirm that aerobic conditions are present throughout the entire plume/plume segment;

- $\quad$ determine if plume(s) are expanding/perturbed, stable, or shrinking;

- determine the location of any receptors (if present) and determine the travel time from the edge of the plume to these receptors.

\section{Key Monitoring Concepts}

You will likely just need a simple set of transect wells along the plume centerline and some sentry wells - looks like the "text book" case due to the simple plume shape in a homogenous aquifer.

The slow hydrogeologic setting may mean the plume is of moderate size. However, a long period of time may be required for the plume to become stable (if it is going to). Thus a long temporal record (i.e., the number of years of monitoring data you have) may be needed to determine plume stability.

\section{Key Uncertainty Concepts}

Because daughter compounds for direct aerobic metabolism of contaminants are not available, it may be difficult to show that this type of attenuation process is occurring. There may be uncertainty about the plume conditions in general under this scenario because the plume may not be fully developed due to the slow flow conditions and it may not be evident if MNA is currently working.

It may also be uncertain whether co-metabolic reactions are occurring in the plume segment. To resolve this uncertainty, it may be necessary to perform a detailed analysis of contaminant loss down the centerline of the plume: i) to determine if the observed reduction in concentrations is due to dispersion only or due to a combination of dispersion and co-metabolic reactions; and ii) to determine if a primary substrate (e.g., phenol, methane, propane, etc.) is present in the plume segment. 


\begin{tabular}{|c|c|c|c|c|}
\hline $\begin{array}{c}\text { Simple } \\
\text { Fast }\end{array}$ & $\begin{array}{c}\text { Simple } \\
\text { Slow }\end{array}$ & $\begin{array}{c}\text { Heterogeneous } \\
\text { Fast }\end{array}$ & $\begin{array}{c}\text { Heterogeneous } \\
\text { Slow }\end{array}$ & $\begin{array}{c}\text { Fractured/ } \\
\text { Porous Rock }\end{array}$ \\
\hline
\end{tabular}

\begin{tabular}{|l|l|l|}
\hline Aerobic & Anoxic & Anaerobic \\
\hline
\end{tabular}

\section{HOW DO I ANALYZE DATA?}

In a slow-flowing aquifer, a plume is more likely to show concentration differences over short distances and the plume will become stable (if it is going to) over a long period of time. Thus, while a good first step in this type of aquifer is to examine plume maps, concentration vs. time at each well, and concentration vs. distance plots to determine whether the plume is attenuating, additional information may be needed. Also, because daughter products are not readily measured for aerobic reactions, additional information to confirm attenuation processes may be needed. Especially if the plume edge is close to receptors, it may be necessary to provide additional data to verify aerobic degradation processes are occurring and to assess the sustainability of these processes. Molecular probe data to verify the presence of the appropriate microorganisms and laboratory microcosm tests may provide this type of information. A more detailed geochemical analysis may also be warranted to assess sustainability. A simple transport model such as BIOCHLOR can be helpful in analyzing and visualizing the data and expected plume conditions for comparison to field data. The chart below summarizes an approach for analyzing data at sites depending on whether the concentration data indicates that the plume is decreasing, stable, or increasing and the source type. As noted in the table, as the source gets stronger and the plume is less likely to be decreasing in extent, more information is needed to support selection of an MNA remedy.

\begin{tabular}{|c|c|c|c|}
\hline & \multicolumn{3}{|c|}{ PLUME STATUS } \\
\hline $\begin{array}{l}\text { CONTAMINANT } \\
\text { CONCENTRATIONS/GEOCHEMICAL } \\
\text { STATUS }\end{array}$ & $\begin{array}{l}\text { DECREASING OR } \\
\text { PROBABLY } \\
\text { DECREASING }\end{array}$ & STABLE & $\begin{array}{l}\text { INCREASING, } \\
\text { PROBABLY } \\
\text { INCREASING, OR } \\
\text { PERTURBED }^{1}\end{array}$ \\
\hline Weak Source & - Mass loss & $\begin{array}{l}\text { - } \text { Mass loss } \\
\text { - } \text { Geochemical footprints }\end{array}$ & $\begin{array}{l}\text { - } \text { Mass loss } \\
\text { - } \text { Geochemical footprints } \\
\text { - } \quad \text { Simple Model }\end{array}$ \\
\hline Strong Source & $\begin{array}{l}\text { - Mass loss } \\
\text { - Geochemical footprints }\end{array}$ & $\begin{array}{l}\text { - } \text { Mass loss } \\
\text { - } \text { Geochemical footprints } \\
\text { - Simple Model }\end{array}$ & $\begin{array}{ll}\text { - } & \text { Mass loss } \\
\text { - } & \text { Geochemical footprints } \\
\text { - } & \text { Comprehensive Model/ } \\
& \text { Special Studies }\end{array}$ \\
\hline
\end{tabular}

${ }^{1}$ For instance if the plume has been impacted by a previous remedy such as P\&T.

To demonstrate mass loss construct these graphics:

- Concentration vs. time plots at individual wells;

- Concentration vs. distance plots, with multiple lines for different sampling events through time;

- Plume maps showing plume extent at different times (i.e., either panel maps, or one map with several plume boundaries for different times).

To show geochemical footprints make tables or figures that show:

- Daughter product production from abiotic reactions;

- Presence of primary substrate for co-metabolic reactions;

- Chloride product (this may not work at many sites, however, due to background chloride);

- Moderate to high dissolved oxygen concentrations (shows geochemical conditions are OK);

- No or limited methane production (shows geochemical conditions are OK).

To perform modeling, typical tools include the following:

- Simple transport model (analytical model, e.g., BIOCHLOR, BIOBALANCE ${ }^{1}$ );

- Comprehensive transport model (numerical model, e.g., RT3D).

If a special study is needed, some of the following may be applicable:

- Carbon/chlorine isotope analysis (indicator of degradation processes)

- Molecular probes (indicators of microbial activity)

- Microcosm tests (determine the reaction processes occurring at the site).

1 Developed by Groundwater Services (www.gsi-net.com), with the support of the Savannah River National Laboratory and $\mathrm{DOE}$, to evaluate monitored natural attenuation at chlorinated solvent sites. 


\begin{tabular}{|c|c|c|c|c|c|c|}
\hline $\begin{array}{c}\text { Simple } \\
\text { Fast }\end{array}$ & $\begin{array}{c}\text { Simple } \\
\text { Slow }\end{array}$ & $\begin{array}{c}\text { Heterogeneous } \\
\text { Fast }\end{array}$ & $\begin{array}{c}\text { Heterogeneous } \\
\text { Slow }\end{array}$ & $\begin{array}{c}\text { Fractured/ } \\
\text { Porous Rock }\end{array}$ \\
\hline
\end{tabular}

\section{WHAT ABOUT COSTS AND ENHANCEMENTS?}

Costs for evaluating and implementing MNA for this scenario are primarily dependent on the following items.

- $\quad$ Source strength - Stronger and longer lasting sources will be more costly.

- $\quad$ Depth to the plume and size of the plume - Deeper, larger plumes require more and costlier monitoring wells.

- $\quad$ Travel time to the receptor - Plumes closer to receptors will require more frequent monitoring and over a long period of time with the slow groundwater flow rate.

- $\quad$ Plume stability - Less stable plumes require more rigorous evaluation and monitoring to select and implement MNA. With the slow groundwater flow rate, more rigorous evaluation or a longer period of monitoring may cause higher costs than for sites with a high groundwater flow rate.

MNA may be a viable single remedy for the site. If it is determined that MNA may not meet remediation goals, a good first option is to evaluate the potential use of sustainable enhancements (enhanced attenuation). The objective being to adjust the attenuation conditions sufficiently such that the plume is controlled. If the enhancements are insufficient to control the plume, source control treatment may be required. In general, enhanced attenuation is less likely a viable option for a fast flow regime with a strong source, unless source treatment is undertaken initially. The sections below discuss options for enhanced attenuation and source control related to this scenario.

\section{Key Enhanced Attenuation Concepts}

Enhancements are presented organized by the different zones in which they may be applied: source zone (reduction of contaminant mass flux to plume); plume (enhanced attenuation processes); or discharge zone (enhanced attenuation processes). Within the source zone, enhancements can be applied as a hydraulic manipulation or as a passive source reduction (active source control is discussed in the next section). Within the plume and discharge zone, either biological (microbial or plant based) or abiotic (abiotic degradation, reactive barriers, sorption) attenuation processes can be enhanced. A description of potential enhanced attenuation approaches, and their applicability to Scenario 6 sites, is shown below. More detailed information about each technology listed below is available in Early et al., (2005). 


\begin{tabular}{|c|c|c|c|c|}
\hline $\begin{array}{c}\text { Simple } \\
\text { Fast }\end{array}$ & $\begin{array}{c}\text { Simple } \\
\text { Slow }\end{array}$ & $\begin{array}{c}\text { Heterogeneous } \\
\text { Fast }\end{array}$ & $\begin{array}{c}\text { Heterogeneous } \\
\text { Slow }\end{array}$ & $\begin{array}{c}\text { Fractured/ } \\
\text { Porous Rock }\end{array}$ \\
\hline
\end{tabular}

\begin{tabular}{|l|c|c|}
\hline \multicolumn{2}{|c}{ GEOCHEMICAL SETTING } \\
\hline Aerobic & Anoxic & Anaerobic \\
\hline
\end{tabular}

\section{Enhancement Summary}

\begin{tabular}{|c|c|c|}
\hline Enhancement & Description & Applicability to Scenario 6 Sites \\
\hline \multicolumn{3}{|c|}{ Source Zone Enhancements } \\
\hline $\begin{array}{l}\text { Surface water or } \\
\text { groundwater } \\
\text { interception/diversion }\end{array}$ & $\begin{array}{l}\text { Use of interception } \\
\text { trenches or wells, } \\
\text { surface covers, or } \\
\text { phyto-covers (plants) } \\
\text { to reduce water flux } \\
\text { through source area }\end{array}$ & $\begin{array}{l}\text { Likely more applicable using surface covers or phyto- } \\
\text { covers if the source is primarily within the vadose zone. } \\
\text { With slow flow rate, this method may be relatively } \\
\text { effective compared to application in higher flow rate } \\
\text { conditions. }\end{array}$ \\
\hline Physical containment & $\begin{array}{c}\text { Use of grout walls and } \\
\text { other physical } \\
\text { containment }\end{array}$ & $\begin{array}{l}\text { Potentially applicable depending on the geometry of the } \\
\text { source zone. }\end{array}$ \\
\hline Passive extraction & $\begin{array}{l}\text { Use of passive soil } \\
\text { vapor extraction }\end{array}$ & Useful if the source is primarily within the vadose zone. \\
\hline $\begin{array}{l}\text { Enhanced } \\
\text { biodegradation }\end{array}$ & $\begin{array}{l}\text { Injection of long-term } \\
\text { dissolved oxygen } \\
\text { source }\end{array}$ & $\begin{array}{l}\text { Well suited for aerobic sites if oxygen concentrations are } \\
\text { marginal in some areas; enhances existing aerobic } \\
\text { biodegradation reactions. Simple hydrogeology makes } \\
\text { application easier. Slow groundwater flow may make } \\
\text { application easier than for high flow rate conditions. }\end{array}$ \\
\hline $\begin{array}{l}\text { Enhanced } \\
\text { biodegradation }\end{array}$ & $\begin{array}{l}\text { Injection of electron } \\
\text { donor (e.g., HRC, } \\
\text { molasses, vegetable } \\
\text { oil) to enhance } \\
\text { microbial degradation } \\
\text { of the source. }\end{array}$ & $\begin{array}{l}\text { Typically more appropriate for anaerobic sites; stimulates } \\
\text { anaerobic contaminant biodegradation reactions. } \\
\text { Potentially useful at aerobic sites in source area to } \\
\text { convert contaminants such as PCE and TCE into } \\
\text { contaminants such as DCE and VC that are degradable } \\
\text { under aerobic conditions. Need careful control of process } \\
\text { to avoid depleting all of the oxygen for the plume and } \\
\text { eliminating the potential for aerobic reactions. Simple } \\
\text { hydrogeology makes application easier. Slow } \\
\text { groundwater flow may make application easier than for } \\
\text { high flow rate conditions. }\end{array}$ \\
\hline $\begin{array}{l}\text { Permeable reactive } \\
\text { barrier }\end{array}$ & $\begin{array}{c}\text { Use of zero valent } \\
\text { iron, reduced } \\
\text { sediment iron, } \\
\text { enhanced partitioning, } \\
\text { or biological barrier to } \\
\text { attenuate some of the } \\
\text { contaminant flux at } \\
\text { the downgradient } \\
\text { edge of the source } \\
\text { area. }\end{array}$ & $\begin{array}{l}\text { Barriers typically use anaerobic reactions. Influent of } \\
\text { dissolved oxygen is problematic for the barrier and the } \\
\text { aquifer down gradient of the barrier will be depleted in } \\
\text { oxygen. Thus, barriers that use anaerobic reactions are } \\
\text { not typically suitable for aerobic sites. Easier to apply in } \\
\text { slow flow regime. Slower groundwater flows potentially } \\
\text { result in less contaminant and competing electron } \\
\text { acceptors passing through the barrier, requiring less } \\
\text { thickness to achieve desired treatment levels. }\end{array}$ \\
\hline
\end{tabular}




\begin{tabular}{|c|c|c|c|c|}
\hline $\begin{array}{c}\text { Simple } \\
\text { Fast }\end{array}$ & $\begin{array}{c}\text { Simple } \\
\text { Slow }\end{array}$ & $\begin{array}{c}\text { Heterogeneous } \\
\text { Fast }\end{array}$ & $\begin{array}{c}\text { Heterogeneous } \\
\text { Slow }\end{array}$ & $\begin{array}{c}\text { Fractured/ } \\
\text { Porous Rock }\end{array}$ \\
\hline
\end{tabular}

\begin{tabular}{|l|l|l|}
\hline Aerobic & Anoxic & Anaerobic \\
\hline
\end{tabular}

\section{Enhancement Summary (Con'td)}

\section{Enhancement}

\section{Description}

Plume and Discharge Zone Enhancements

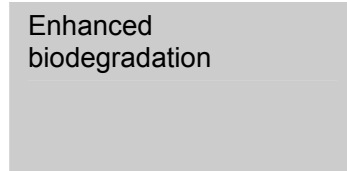

Enhanced biodegradation

Permeable reactive
barrier

Phytoextraction

Plant-based hydraulic control (plume enhancement only)

Injection of long-term
dissolved oxygen
source

Injection of electron donor (e.g., HRC, molasses, vegetable oil) to enhance microbial degradation of the source.

\section{Use of zero valent iron, reduced sediment iron,} enhanced partitioning, or biological barrier to attenuate some of the contaminant flux within the plume.

Use of plants to extract contaminants from near surface groundwater

Use of plants to control hydraulic gradient and slow groundwater
Well suited for aerobic sites if oxygen concentrations are marginal in some areas; enhances existing aerobic biodegradation reactions. Simple hydrogeology makes application easier. Slow groundwater flow may make application easier than for high flow rate conditions.

Typically more appropriate for anaerobic sites; stimulates anaerobic contaminant biodegradation reactions. Potentially useful at aerobic sites in source area to convert contaminants such as PCE and TCE into contaminants such as DCE and VC that are degradable under aerobic conditions. Need careful control of process to avoid depleting all of the oxygen for the plume and eliminating the potential for aerobic reactions. Simple hydrogeology makes application easier. Slow groundwater flow may make application easier than for high flow rate conditions.

Barriers typically use anaerobic reactions. Influent of dissolved oxygen is problematic for the barrier and the aquifer down gradient of the barrier will be depleted in oxygen. Thus, barriers that use anaerobic reactions are not typically suitable for aerobic sites. Easier to apply in slow flow regime. Slower groundwater flows potentially result in less contaminant and competing electron acceptors passing through the barrier, requiring less thickness to achieve desired treatment levels.

May be effective in slow groundwater flow conditions.

May be effective in slow groundwater flow conditions. 


\begin{tabular}{|c|c|c|c|c|}
\hline $\begin{array}{c}\text { Simple } \\
\text { Fast }\end{array}$ & $\begin{array}{c}\text { Simple } \\
\text { Slow }\end{array}$ & $\begin{array}{c}\text { Heterogeneous } \\
\text { Fast }\end{array}$ & $\begin{array}{c}\text { Heterogeneous } \\
\text { Slow }\end{array}$ & $\begin{array}{c}\text { Fractured/ } \\
\text { Porous Rock }\end{array}$ \\
\hline
\end{tabular}

\begin{tabular}{|l|c|c|}
\hline \multicolumn{2}{|c}{ GEOCHEMICAL SETTING } \\
\hline Aerobic & Anoxic & Anaerobic \\
\hline
\end{tabular}

\section{Key Source Control Concepts}

A description of potential source control measures, and their applicability to Scenario 6 sites, is shown below. Note that source control measures are unlikely to achieve complete restoration at a site, and some source material is always left behind after treatment (U.S. EPA, 2003; McGuire et al., 2006).

\begin{tabular}{|c|c|c|c|}
\hline$\frac{\text { Example }}{\text { Technology }}$ & $\begin{array}{l}\text { Performance } \\
\left(25^{\mathrm{th}}-75^{\text {th }} \text { Percentile }\right. \\
\% \text { reduction in } \\
\text { parent compound })^{1}\end{array}$ & $\begin{array}{l}\frac{\text { Unit Cost }}{25^{\text {th }}-75^{\text {th }}} \\
\text { Percentile } \\
(\$ / y r d)^{2}\end{array}$ & Applicability to Scenario 6 Sites \\
\hline In-situ biodegradation & $73-99$ & $27-152$ & $\begin{array}{l}\text { Potentially well suited for aerobic sites through } \\
\text { addition of co-substrate for aerobic degradation or } \\
\text { potentially through use of anaerobic reactions } \\
\text { depending on how this action impacts the } \\
\text { downgradient geochemical conditions. Simple } \\
\text { hydrogeology makes application easier. Slow } \\
\text { groundwater flow may make application easier than } \\
\text { for high flow rate conditions. Least expensive } \\
\text { treatment option. }\end{array}$ \\
\hline Thermal treatment & $68-99.9$ & $48-129$ & Does not appear to disrupt MNA after treatment. \\
\hline Chemical oxidation & $70-97$ & $47-194$ & $\begin{array}{l}\text { Removes more total CVOCs than enhanced } \\
\text { biodegradation but shows more rebound }{ }^{1} \text {. Simple } \\
\text { hydrogeology makes application easier. May be } \\
\text { suitable for aerobic sites. }\end{array}$ \\
\hline Surfactant/cosolvents & $92-98$ & $118-1322$ & $\begin{array}{l}\text { High treatment efficiency but much higher cost. } \\
\text { Costs reflect some expensive pilot-scale projects. } \\
\text { Simple hydrogeology makes application easier. } \\
\text { Some surfactants/cosolvents can serve as electron } \\
\text { donors for subsequent anaerobic biodegradation } \\
\text { reactions. }\end{array}$ \\
\hline Air sparging & - & - & $\begin{array}{l}\text { May be a viable alternative depending on the site } \\
\text { geology (e.g., contamination in an unconfined } \\
\text { aquifer). }\end{array}$ \\
\hline $\begin{array}{l}\text { Pump and Treat } \\
\text { source containment }\end{array}$ & NA & & $\begin{array}{l}\text { Due to the low groundwater flow rate, a relatively } \\
\text { small system may be sufficient. This approach } \\
\text { does not reduce mass significantly compared to the } \\
\text { rate of mass loss without P\&T and may need to be } \\
\text { operated for a long time. }\end{array}$ \\
\hline
\end{tabular}




\begin{tabular}{|c|c|c|c|c|}
\hline $\begin{array}{c}\text { Simple } \\
\text { Fast }\end{array}$ & $\begin{array}{c}\text { Simple } \\
\text { Slow }\end{array}$ & $\begin{array}{c}\text { Heterogeneous } \\
\text { Fast }\end{array}$ & $\begin{array}{c}\text { Heterogeneous } \\
\text { Slow }\end{array}$ & $\begin{array}{c}\text { Fractured/ } \\
\text { Porous Rock }\end{array}$ \\
\hline
\end{tabular}

\begin{tabular}{|l|l|l|}
\hline Aerobic & Anoxic & Anaerobic \\
\hline
\end{tabular}

\section{SCENARIO NUMBER 7}

Faster Flow With Significant Heterogeneities and Anaerobic
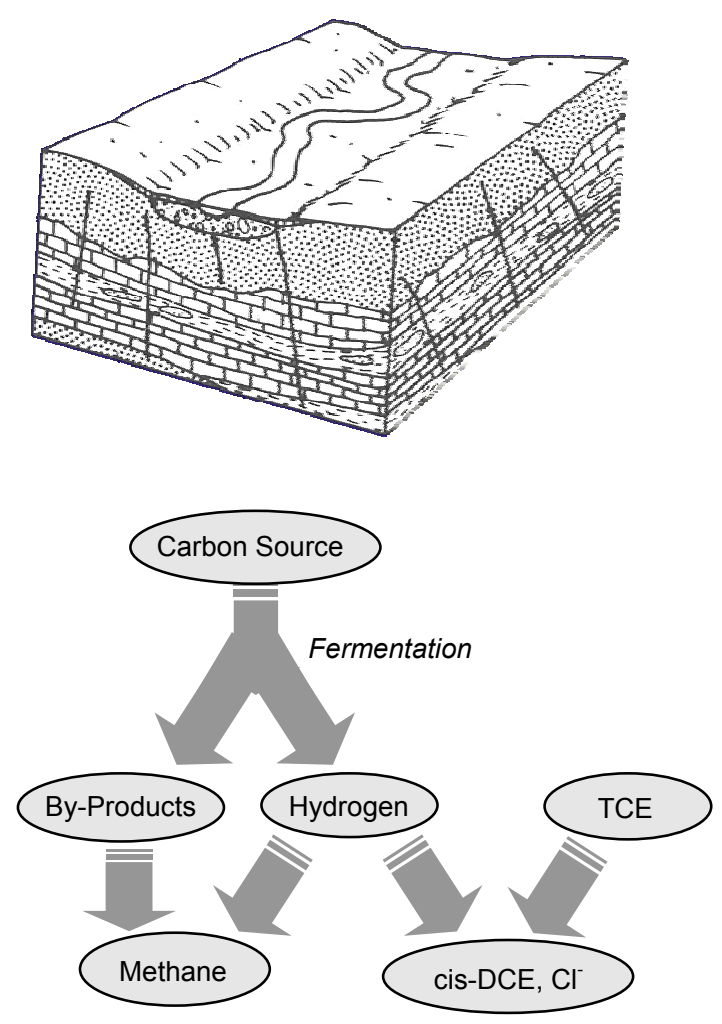


\begin{tabular}{|c|c|c|c|c|}
\hline $\begin{array}{c}\text { Simple } \\
\text { Fast }\end{array}$ & $\begin{array}{c}\text { Simple } \\
\text { Slow }\end{array}$ & $\begin{array}{c}\text { Heterogeneous } \\
\text { Fast }\end{array}$ & $\begin{array}{c}\text { Heterogeneous } \\
\text { Slow }\end{array}$ & $\begin{array}{c}\text { Fractured/ } \\
\text { Porous Rock }\end{array}$ \\
\hline
\end{tabular}

GEOCHEMICAL SETTING

\section{SCENARIO 7 DESCRIPTION: FAST FLOW WITH SIGNIFICANT HETEROGENEITIES AND ANAEROBIC}

The hydrogeologic setting and geochemical setting below define the basic scenario type. This scenario has the following characteristics:

\section{Hydrogeologic Setting}

"Faster Flow With Significant Heterogeneities" Hydrogeology:

- Potentially multiple hydrogeologic units

- Wide distribution of hydraulic conductivity

- Relatively high groundwater seepage velocity

(see Section 2.1 and Appendix 1 for more information)

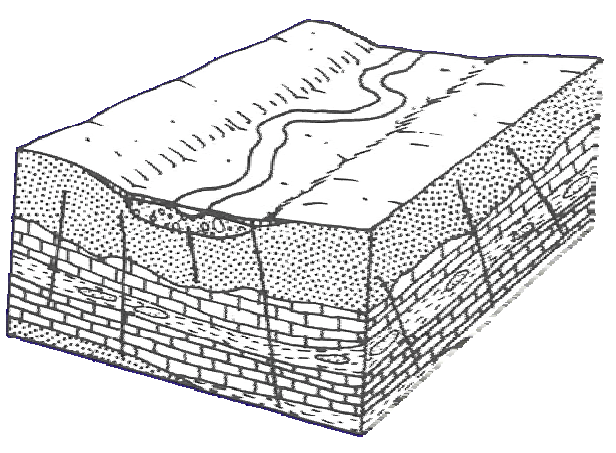

Block Diagram of River Alluvium with Overbank Deposits Aquifer from DRASTIC System

\section{Geochemical Setting}

"Anaerobic" Geochemistry:

- Dissolved oxygen and redox are low

- Low to moderate concentrations of competing electron acceptors (nitrate, sulfate)

- Methane being produced.

(see Sections 2.2 for more information)

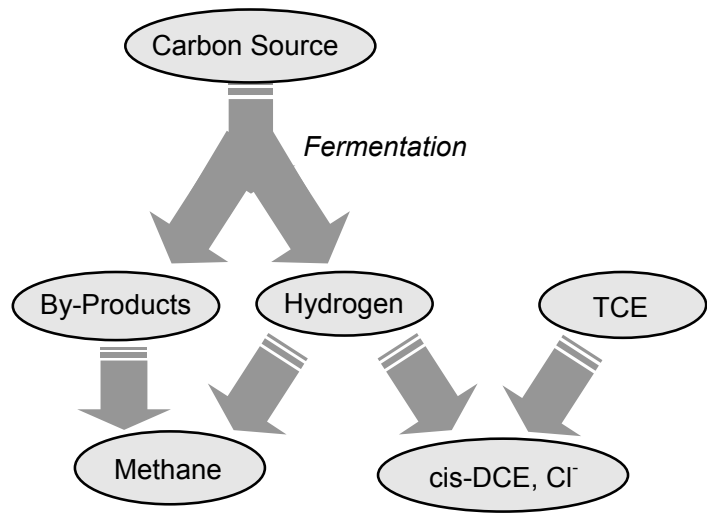

Example Reactions for "Anaerobic" Geochemical Setting 
WSRC-STI-2006-00096, Rev. 1

October 2, 2006

HYDROGEOLOGIC SETTING

GEOCHEMICAL SETTING

\begin{tabular}{|c|c|c|c|c|c|c|c|}
\hline $\begin{array}{l}\text { Simple } \\
\text { Fast }\end{array}$ & $\begin{array}{l}\text { Simple } \\
\text { Slow }\end{array}$ & $\begin{array}{c}\text { Heterogeneous } \\
\text { Fast }\end{array}$ & $\begin{array}{c}\text { Heterogeneous } \\
\text { Slow }\end{array}$ & $\begin{array}{c}\text { Fractured/ } \\
\text { Porous Rock }\end{array}$ & Aerobic & Anoxic & Anaerobic \\
\hline
\end{tabular}

\section{KEY DECHLORINATION REACTIONS}

\section{Reaction Overview}

The chart to the right shows which reactions are likely to occur, which occur but at a slow rate, which may occur under specific conditions, and which are unlikely to occur.

\section{Typically Biodegradable Parent Compounds}

These compounds are typically degradable under anaerobic conditions:

- PCE

- TCE

- 1,1,1-TCA

- 1,2-DCA

- $\quad \mathrm{CT}$

\section{Typical Daughter Products}

Daughter products that may be present depending on the parent compound and the reactions listed to the right:

- TCE

- cis 1,2-DCE

- VC

- 1,2-DCA

- 1,1-DCE

- $\mathrm{CF}$

See Section 5.1 for more information about reactions

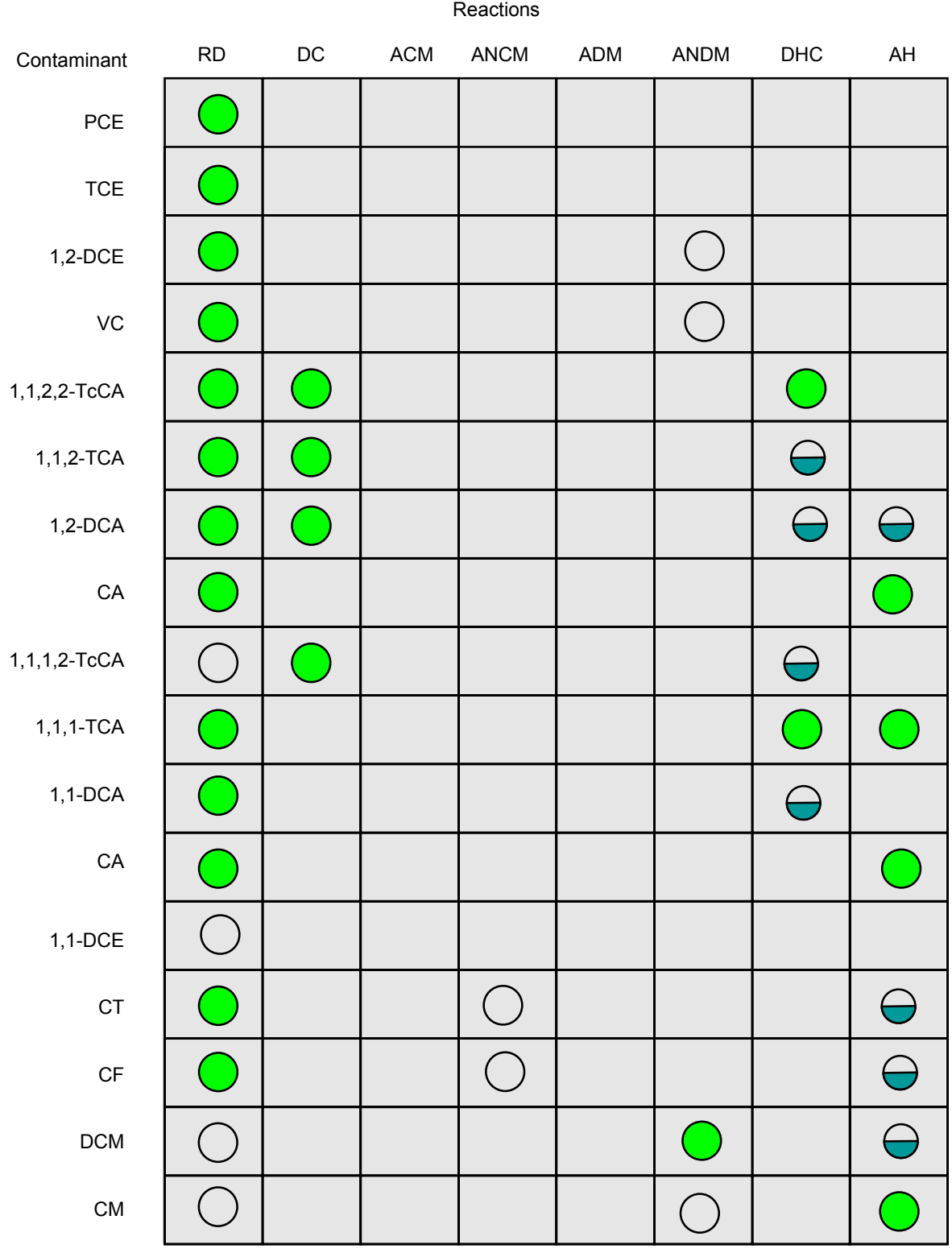

REACTIONS

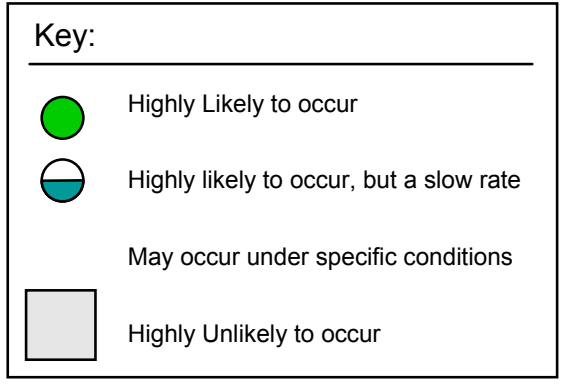

\begin{tabular}{|c|l|}
\hline ACM & Aerobic Co-Metabolism \\
\hline ANCM & Anaerobic Co-Metabolism \\
\hline ADM & Aerobic Direct Metabolism \\
\hline ANDM & Anaerobic Direct Metabolism \\
\hline DHC & Dehydrochlorination (abiotic) \\
\hline AH & Abiotic Hydrolysis \\
\hline DC & Dichloroelimination (biotic) \\
\hline RD & Reductive Dechlorination (hydrogenolysis) \\
\hline
\end{tabular}




\begin{tabular}{|c|c|c|c|c|}
\hline $\begin{array}{c}\text { Simple } \\
\text { Fast }\end{array}$ & $\begin{array}{c}\text { Simple } \\
\text { Slow }\end{array}$ & $\begin{array}{c}\text { Heterogeneous } \\
\text { Fast }\end{array}$ & $\begin{array}{c}\text { Heterogeneous } \\
\text { Slow }\end{array}$ & $\begin{array}{c}\text { Fractured/ } \\
\text { Porous Rock }\end{array}$ \\
\hline
\end{tabular}

\begin{tabular}{|l|l|l|}
\hline Aerobic & Anoxic & Anaerobic \\
\hline
\end{tabular}

\section{EFFECT OF MODIFYING FACTORS}

\begin{tabular}{|c|c|}
\hline MODIFYING FACTOR & KEY POINTS \\
\hline Source Strength & $\begin{array}{l}\text { IF YOU HAVE A STRONG SOURCE: } \\
\text { - } \quad \text { Plumes (both parent and daughter compounds) may be longer } \\
\text { - } \quad \text { Source zones may persist for longer periods of time } \\
\text { - More likely to need EA or source control measures } \\
\text { IF YOU HAVE A MODERATE SOURCE: } \\
\text { - Intermediate condition between Strong and Weak Source } \\
\text { IF YOU HAVE A WEAK SOURCE: } \\
\text { - Plumes may be shorter } \\
\text { - } \quad \text { Source zones may not persist as long } \\
\text { - } \quad \text { MNA alone or MNA with EA more likely to be sufficient }\end{array}$ \\
\hline Source & $\begin{array}{l}\text { IF YOU HAVE MOSTLY A VADOSE ZONE SOURCE: } \\
\text { - Source may appear small due to dilution but can be large } \\
\text { - Sources in clay vadose zones will be weaker but more long-lived than } \\
\text { sandy vadose zone } \\
\text { - Plumes will be thinner and closer to water table } \\
\text { IF YOU HAVE A SUBMERGED SOURCE: } \\
\text { - Complex hydrogeology means matrix diffusion may be important } \\
\text { - Source mass flux can decrease relatively rapidly as DNAPL fingers } \\
\text { - } \quad \text { dissolve } \\
\text { IF YOU HAVE A MIXED SOURCE: } \\
\text { - May have combination of the factors above }\end{array}$ \\
\hline Travel Tim & $\begin{array}{l}\text { CLOSE RECEPTORS (<2 YEARS TRAVEL TIME) } \\
\text { - More intensive monitoring system likely to be needed due to potential } \\
\text { - } \\
\text { Merious consequences in event of failure of MNA/EA. } \\
\text { MODERATE RECEPTORS ( }>2 \text { but }<5 \text { YEARS TRAVEL TIME) } \\
\text { - Represents middle-ground case } \\
\text { DISTANT RECEPTORS ( }>5 \text { YEARS TRAVEL TIME) } \\
\text { - Less intensive monitoring system likely to be needed due to lower } \\
\text { - } \quad \text { MNtential for serious consequences in event of failure of MNA/EA. }\end{array}$ \\
\hline Plume Stability & $\begin{array}{l}\text { EXPANDING OR PERTURBED }{ }^{1} \text { PLUME } \\
-\quad \text { More intensive monitoring system likely to be needed } \\
\text { STABLE PLUME } \\
\text { - Represents middle-ground case } \\
\text { SHRINKING PLUME } \\
\text { - Less intensive monitoring system likely to be needed } \\
\text { - MNA alone likely to be sufficient } \\
{ }^{1} \text { For instance if the plume has been impacted by a previous remedy such as P\&T. }\end{array}$ \\
\hline
\end{tabular}




\begin{tabular}{|c|c|c|c|c|c|c|c|}
\hline $\begin{array}{c}\text { Simple } \\
\text { Fast }\end{array}$ & $\begin{array}{c}\text { Simple } \\
\text { Slow }\end{array}$ & $\begin{array}{c}\text { Heterogeneous } \\
\text { Fast }\end{array}$ & $\begin{array}{c}\text { Heterogeneous } \\
\text { Slow }\end{array}$ & $\begin{array}{c}\text { Fractured/ } \\
\text { Porous Rock }\end{array}$ \\
\end{tabular}

\section{WILL MNA WORK?}

\section{Potential for MNA Processes to Control Plume}

This scenario often is well suited for natural attenuation processes to manage the contaminants in the plume or plume segment. The anaerobic conditions almost always mean that biodegradation processes are active.

In a fast-flowing heterogeneous aquifer where anaerobic conditions are present uniformly throughout the plume, relatively high rates of contaminant degradation may be needed to stabilize the plume. With the typical reductive dechlorination processes that are occurring under these conditions, it would be expected that the daughter product plume would be larger than the parent product plume because the degradation rate of daughters is typically slower than the rate for the parents.

The plume shape will be controlled by the heterogeneities in the plume segment. Plumes can be difficult to delineate, and can have complex, 3-dimensional shapes. Matrix diffusion effects in low-permeability zones can result in slowerthan-expected plume growth, which can be mistaken for mass destruction.

At some Scenario 7 sites, "DCE stall" may be of concern. DCE stall is an informal term typically used to describe conditions at chlorinated ethene sites where the cis-1,2-DCE "stalls outs" or exhibits a very low conversion rate to VC. This DCE "stall" condition has been ascribed to a variety of factors, including:

- $\quad$ Lack of the necessary microbiological communities that are required to degrade cis-1,2-DCE to VC;

- The direct conversion of cis-1,2-DCE to carbon dioxide, which makes it appear that cis-1,2-DCE is not being biodegraded because VC is not being produced; but in fact the cis-1,2-DCE is being biodegraded by direct oxidation to carbon dioxide;

- Conditions which are anaerobic enough to support the conversion of TCE to cis-1,2-DCE but not anaerobic enough to support the conversion from cis-1,2-DCE to VC by reductive dechlorination;

- Toxicity effects caused at sites where sulfate reducers are producing hydrogen sulfide $\left(\mathrm{H}_{2} \mathrm{~S}\right)$, but the $\mathrm{H}_{2} \mathrm{~S}$ is not being precipitated fast enough by ferrous iron (a by-product of ferric iron reduction) to prevent toxicity effects in the cis-1,2-DCE degraders.

While the cause of cis-1,2-DCE stall is still being evaluated by a number of researchers, the main implication is that at some chlorinated ethene sites, cis-1,2-DCE plumes are expanding and not being controlled. DCE "stall" does not affect long-term sustainability of a reaction, but does determine if natural attenuation processes are sufficient to prevent migration of the plume.

\section{Key Sustainability Concept}

Fast-flowing plume segments can have high mass flux of contaminants leaving the source, and therefore high rates of degradation are often needed to attenuate the plume. Sufficient natural substrate (e.g., organic matter) or cocontaminants that serve as electron donors and can act as a substrate are needed to sustain high rates of degradation. In a heterogeneous system, care should be taken to consider whether there are conduits for contaminant migration that do not have sufficient substrate available (e.g., a clean sand layer). The BIOBALANCE software system ${ }^{1}$ has a module designed to evaluate sustainability issues for anaerobic MNA reactions. Key input data are: i) mass fraction of solvents vs. donors in NAPL; OR ii) dissolved-phase concentrations of solvents and donors in the source zone.

${ }^{1}$ Developed by Groundwater Services (www.gsi-net.com), with the support of the Savannah River National Laboratory and DOE, to evaluate monitored natural attenuation at chlorinated solvent sites. 


\begin{tabular}{|c|c|c|c|c|c|c|c|}
\hline $\begin{array}{c}\text { Simple } \\
\text { Fast }\end{array}$ & $\begin{array}{c}\text { Simple } \\
\text { Slow }\end{array}$ & $\begin{array}{c}\text { Heterogeneous } \\
\text { Fast }\end{array}$ & $\begin{array}{c}\text { Heterogeneous } \\
\text { Slow }\end{array}$ & $\begin{array}{c}\text { Fractured/ } \\
\text { Porous Rock }\end{array}$ \\
\hline
\end{tabular}

\section{HOW DO I CHARACTERIZE THIS TYPE OF SITE?}

\section{Actions Needed to Determine MNA Viability:}

The following are key considerations for evaluating the viability of MNA at a Fast Flow With Significant Heterogeneities and Anaerobic type site:

- $\quad$ both parent compound and daughter compounds need to be delineated (the anaerobic setting means that a number of daughter products will likely be generated);

- $\quad$ confirm that anaerobic conditions are present throughout the entire plume segment;

- $\quad$ determine the relative horizontal and vertical plume movement and whether there are layers (e.g., sandy units) where the plume movement is significantly greater than in other parts of the aquifer;

- $\quad$ determine if plume(s) are expanding/perturbed, stable, or shrinking;

- determine the location of any receptors (if present) and determine the travel time from the edge of the plume to these receptors;

- a mass-balance type evaluation of the source zone will help to determine if the electron donor supply is sustainable over the long term.

\section{Key Monitoring Concepts}

The fast hydrogeologic setting may mean the plume can be relatively large, and therefore require more monitoring points. In addition, the plume can become stable (if it is going to) more quickly than for a slow hydrogeologic setting, so an extremely long temporal record (i.e., the number of years of monitoring data you have) may not be needed to determine plume stability.

A more extensive monitoring system will likely be required to delineate the plume, because heterogeneities can result in wide distribution of hydraulic conductivity across the plume segment. Attention to vertical characteristics of the plume and monitoring within specific hydrologic layers may be particularly important. Plumes can have unusual shapes, such as apparent cross-gradient (regional gradient) flow patterns. This type of hydrogeologic setting benefits from plume delineation strategies using direct push approaches and adaptive plume delineation strategies.

\section{Key Uncertainty Concepts}

A key uncertainty for this scenario is associated with knowing that the plume is sufficiently delineated. In a heterogeneous subsurface, appropriate selection of monitoring locations is more difficult both horizontally and vertically within the plume. Because all of the MNA evaluation methods rely on a suitable conceptual model and data to describe the plume and subsurface properties, more data is typically required in a heterogeneous system to complete the evaluation at a level of detail acceptable to decision makers. Another uncertainty may be the sustainability of MNA due to the high mass flux of the source that may be present (see key sustainability concept section). 


\begin{tabular}{|c|c|c|c|c|}
\hline $\begin{array}{c}\text { Simple } \\
\text { Fast }\end{array}$ & $\begin{array}{c}\text { Simple } \\
\text { Slow }\end{array}$ & $\begin{array}{c}\text { Heterogeneous } \\
\text { Fast }\end{array}$ & $\begin{array}{c}\text { Heterogeneous } \\
\text { Slow }\end{array}$ & $\begin{array}{c}\text { Fractured/ } \\
\text { Porous Rock }\end{array}$ \\
\hline
\end{tabular}

\begin{tabular}{|l|c|c|}
\hline \multicolumn{2}{|c|}{ GEOCHEMICAL SETTING } \\
\hline Aerobic & Anoxic & Anaerobic \\
\hline
\end{tabular}

\section{HOW DO I ANALYZE DATA?}

In a fast-flowing aquifer, a plume is more likely to be long and will become stable (if it is going to) in a shorter period of time than in slower-flow aquifers. Thus, plume maps, concentration vs. time at each well, and concentration vs. distance plots may be useful to determine if the plume is expanding, stable, or shrinking. A relatively short temporal record of concentration at key wells (particularly at the leading edge of the plume) may be sufficient to evaluate MNA under the heterogeneous-fast flowing scenario. However, care must be taken to ensure that the monitoring network is sufficient for the heterogeneous conditions at the site. Concentrations should show an increase in the ratio of daughter to parent products with distance. If the plume edge is close to receptors, it may be necessary to provide additional data to verify anaerobic degradation processes are occurring and to assess the sustainability of these processes. Molecular probe data to verify the presence of the appropriate microorganisms and laboratory microcosm tests may provide this type of information. A more detailed geochemical analysis may also be warranted to assess sustainability.

Transport models can be very helpful in analyzing and visualizing the data and expected plume conditions for comparison to field data. Transport models may be needed to predict the future state of the plume so that decisions can be made in a timely fashion and then confirmed through the long-term monitoring portion of MNA implementation. A simple transport model such as BIOCHLOR can be helpful as a screening tool for analyzing and visualizing the data and expected plume conditions for comparison to field data. However, BIOCHLOR will not be sufficient to describe the flow conditions in a heterogeneous aquifer. As such, more complex numerical modeling is more likely to be needed as part of MNA evaluation for Scenario 7 sites. To support this more complex analysis, more detailed field measurements of hydraulic conditions may be required. The chart below summarizes an approach for analyzing data at sites depending on whether the concentration data indicates that the plume is decreasing, stable, or increasing and the source type. As noted in the table, as the source gets stronger and the plume is less likely to be decreasing in extent, more information is needed to support selection of an MNA remedy.

\begin{tabular}{|c|c|c|c|}
\hline & \multicolumn{3}{|c|}{ PLUME STATUS } \\
\hline $\begin{array}{l}\text { CONTAMINANT } \\
\text { CONCENTRATIONS/GEOCHEMICAL } \\
\text { STATUS }\end{array}$ & $\begin{array}{l}\text { DECREASING OR } \\
\text { PROBABLY } \\
\text { DECREASING }\end{array}$ & STABLE & $\begin{array}{l}\text { INCREASING, } \\
\text { PROBABLY } \\
\text { INCREASING, OR } \\
\text { PERTURBED }^{1}\end{array}$ \\
\hline Weak Source & $\begin{array}{l}\text { - } \text { Mass loss } \\
\text { - } \text { Geochemical footprints }\end{array}$ & $\begin{array}{l}\text { - } \text { Mass loss } \\
\text { - } \quad \text { Simple Model }\end{array}$ & $\begin{array}{l}\text { - Mass loss } \\
\text { - Geochemical footprints } \\
\text { - Comprehensive Model/ } \\
\text { Special Studies }\end{array}$ \\
\hline Strong Source & $\begin{array}{ll}\text { - } & \text { Mass loss } \\
\text { - } & \text { Geochemical footprints } \\
\text { - } & \text { Simple Model }\end{array}$ & $\begin{array}{l}\text { - Mass loss } \\
\text { - } \text { Geochemical footprints } \\
\text { Special Studies }\end{array}$ & $\begin{array}{l}\text { - Mass loss } \\
\text { - Geochemical footprints } \\
\text { - Comprehensive Model/ } \\
\text { Special Studies }\end{array}$ \\
\hline
\end{tabular}

${ }^{1}$ For instance if the plume has been impacted by a previous remedy such as P\&T.

To demonstrate mass loss construct these graphics:

- Concentration vs. time plots at individual wells;

- Concentration vs. distance plots, with multiple lines for different sampling events through time;

- Plume maps showing plume extent at different times (i.e., either panel maps, or one map with several plume boundaries for different times).

To show geochemical footprints make tables or figures that show:

- Daughter product production;

- Ethene/Ethane production;

- Chloride production (this may not work at many sites, however, due to background chloride);

- Low dissolved oxygen (shows geochemical conditions are ok);

- Methane and iron(II) distribution (indicators of anaerobic activity);

- Nitrate and sulfate distribution (indicators of competing electron acceptors);

To perform modeling, typical tools include the following:

- Simple transport model (analytical model, e.g., BIOCHLOR, BIOBALANCE ${ }^{1}$ );

- Comprehensive transport model (numerical model, e.g., RT3D). 


\begin{tabular}{|c|c|c|c|c|c|}
\hline $\begin{array}{c}\text { Simple } \\
\text { Fast }\end{array}$ & $\begin{array}{c}\text { Simple } \\
\text { Slow }\end{array}$ & $\begin{array}{c}\text { Heterogeneous } \\
\text { Fast }\end{array}$ & $\begin{array}{c}\text { Heterogeneous } \\
\text { Slow }\end{array}$ & $\begin{array}{c}\text { Fractured/ } \\
\text { Porous Rock }\end{array}$ & Aerobic \\
\hline
\end{tabular}

If a special study is needed, some of the following may be applicable:

- Carbon/chlorine isotope analysis (indicator of degradation processes)

- Molecular probes (indicators of microbial activity)

- Microcosm tests (determine the reaction processes occurring at the site).

${ }^{1}$ Developed by Groundwater Services (www.gsi-net.com), with the support of the Savannah River National Laboratory and DOE, to evaluate monitored natural attenuation at chlorinated solvent sites.

\section{WHAT ABOUT COSTS AND ENHANCEMENTS?}

Costs for evaluating and implementing MNA for this scenario are primarily dependent on the following items.

- $\quad$ Source strength - Stronger and longer lasting sources will be more costly especially due to the high groundwater flow rate.

- $\quad$ Depth to the plume and size of the plume - Deeper, larger plumes require more and costlier monitoring wells.

- $\quad$ Nature of Heterogeneities - More heterogeneous aquifers may require a larger number of monitoring locations and more detailed analysis of flow and transport as part of evaluating MNA.

- $\quad$ Travel time to the receptor - Plumes closer to receptors will require more frequent monitoring especially with the high groundwater flow rate.

- $\quad$ Plume stability - Less stable plumes require more rigorous evaluation and monitoring to select and implement MNA especially with the high groundwater flow rate.

MNA may be a viable single remedy for the site. If it is determined that MNA may not meet remediation goals, a good first option is to evaluate the potential use of sustainable enhancements (enhanced attenuation). The objective being to adjust the attenuation conditions sufficiently such that the plume is controlled. If the enhancements are insufficient to control the plume, source control treatment may be required. In general, enhanced attenuation is less likely a viable option for a fast flow regime with a strong source, unless source treatment is undertaken initially. The sections below discuss options for enhanced attenuation and source control related to this scenario.

\section{Key Enhanced Attenuation Concepts}

Enhanced attenuation must be carefully designed in a heterogeneous flow system. Application of enhancements may be difficult due to the fast groundwater flow conditions. Enhancements are presented organized by the different zones in which they may be applied: source zone (reduction of contaminant mass flux to plume); plume (enhanced attenuation processes); or discharge zone (enhanced attenuation processes). Within the source zone, enhancements can be applied as a hydraulic manipulation or as a passive source reduction (active source control is discussed in the next section). Within the plume and discharge zone, either biological (microbial or plant based) or abiotic (abiotic degradation, reactive barriers, sorption) attenuation processes can be enhanced. A description of potential enhanced attenuation approaches, and their applicability to Scenario 7 sites, is shown below. More detailed information about each technology listed below is available in Early et al., (2005). 
WSRC-STI-2006-00096, Rev. 1

October 2, 2006

HYDROGEOLOGIC SETTING

\begin{tabular}{|c|c|c|c|c|}
\hline $\begin{array}{c}\text { Simple } \\
\text { Fast }\end{array}$ & $\begin{array}{c}\text { Simple } \\
\text { Slow }\end{array}$ & $\begin{array}{c}\text { Heterogeneous } \\
\text { Fast }\end{array}$ & $\begin{array}{c}\text { Heterogeneous } \\
\text { Slow }\end{array}$ & $\begin{array}{c}\text { Fractured/ } \\
\text { Porous Rock }\end{array}$ \\
\hline
\end{tabular}

\begin{tabular}{|l|c|c|}
\hline \multicolumn{2}{|c|}{ GEOCHEMICAL SETTING } \\
\hline Aerobic & Anoxic & Anaerobic \\
\hline
\end{tabular}

\section{Enhancement Summary}

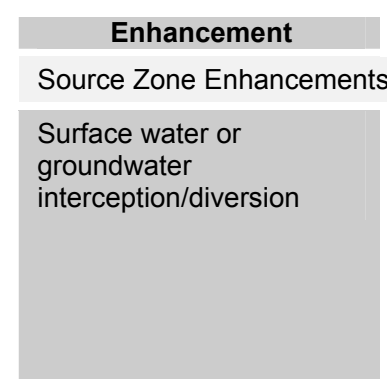

Physical containment

Passive extraction

Permeable reactive barrier

Plume and Discharge Zone Enhancements

Enhanced biodegradation

Permeable reactive barrier

Phytoextraction

Plant-based hydraulic control (plume enhancement only)

Description

Use of interception trenches or wells, surface covers, or phyto-covers (plants) to reduce water flux through source area and/or divert unwanted

Use of grout walls and other physical containment

Use of passive soil

Injection of electron donor (e.g., HRC, molasses, vegetable oil) to enhance microbial degradation of the source.

Use of zero valent iron, reduced sediment iron, enhanced partitioning, or biological barrier to attenuate some of the contaminant flux at the downgradient edge of the source area. electron acceptors. vapor extraction

May be more difficult in high flow rate conditions. May be difficult in heterogeneous conditions. Likely more applicable using surface covers or phyto-covers if the source is primarily within the vadose zone.

Potentially applicable depending on the geometry of the source zone.

Useful if the source is primarily within the vadose zone.

Well suited for anaerobic sites; enhances existing biodegradation reactions. Suitable for most Scenario 7 sites. Complex hydrogeology can make application difficult. Faster groundwater flow may require larger amounts of electron donor than in slower groundwater if electron acceptors are carried into the treatment zone.

May be more difficult and expensive to construct due to heterogeneous conditions. May be more difficult and expensive to construct due to fast flow regime. Faster groundwater flows potentially result in more contaminant and competing electron acceptors passing through the barrier, requiring greater thickness to achieve desired treatment levels. Anaerobic conditions are helpful because oxygen concentration is low and will not disrupt barriers using anaerobic reactions.

Well suited for anaerobic sites; enhances existing biodegradation reactions. Suitable for most Scenario 7 sites. Complex hydrogeology can make application difficult. Faster groundwater flow may require larger amounts of electron donor than in slower groundwater if electron acceptors are carried into the treatment zone.

May be more difficult and expensive to construct due to heterogeneous conditions. May be more difficult and expensive to construct due to fast flow regime. Faster groundwater flows potentially result in more contaminant and competing electron acceptors passing through the barrier requiring greater thickness to achieve desired treatment levels. Anaerobic conditions are helpful because oxygen concentration is low and will not disrupt barriers using anaerobic reactions.

Use of plants to extract contaminants from near surface groundwater

Use of plants to control hydraulic gradient and slow groundwater
Heterogeneous aquifer conditions may make application difficult. May be less effective for fast groundwater flow conditions.

Heterogeneous aquifer conditions may make application difficult. May be less effective for fast groundwater flow conditions. 
WSRC-STI-2006-00096, Rev. 1

October 2, 2006

HYDROGEOLOGIC SETTING

\begin{tabular}{|c|c|c|c|c|}
\hline $\begin{array}{c}\text { Simple } \\
\text { Fast }\end{array}$ & $\begin{array}{c}\text { Simple } \\
\text { Slow }\end{array}$ & $\begin{array}{c}\text { Heterogeneous } \\
\text { Fast }\end{array}$ & $\begin{array}{c}\text { Heterogeneous } \\
\text { Slow }\end{array}$ & $\begin{array}{c}\text { Fractured/ } \\
\text { Porous Rock }\end{array}$ \\
\hline
\end{tabular}

\begin{tabular}{|l|c|c|}
\hline \multicolumn{2}{|c}{ GEOCHEMICAL SETTING } \\
\hline Aerobic & Anoxic & Anaerobic \\
\hline
\end{tabular}

\section{Key Source Control Concepts}

A description of potential source control measures, and their applicability to Scenario 7 sites, is shown below. Note that source control measures are unlikely to achieve complete restoration at a site, and some source material is always left behind after treatment (U.S. EPA, 2003; McGuire et al., 2006).

\begin{tabular}{|c|c|c|c|}
\hline Example Technology & $\begin{array}{l}\text { Performance } \\
\left(25^{\mathrm{th}}-75^{\mathrm{th}} \text { Percentile }\right. \\
\% \text { reduction in } \\
\text { parent compound })^{1}\end{array}$ & $\begin{array}{l}\frac{\text { Unit Cost }}{25^{\text {th }}-75^{\text {th }}} \\
\text { Percentile } \\
\text { (\$/yrd })^{2}\end{array}$ & Applicability to Scenario 7 Sites \\
\hline In-situ biodegradation & $73-99$ & $27-152$ & $\begin{array}{l}\text { Well suited for anaerobic sites; enhances existing } \\
\text { biodegradation reactions. Suitable for most Scenario } 7 \\
\text { sites. Complex hydrogeology can make application } \\
\text { difficult. Faster groundwater flow may require larger } \\
\text { amounts of electron donor than in slower groundwater if } \\
\text { electron acceptors are carried into the treatment zone. }\end{array}$ \\
\hline Thermal treatment & 68-99.9 & $48-129$ & $\begin{array}{l}\text { Does not appear to disrupt MNA after treatment. } \\
\text { Heterogeneous aquifer conditions may make application } \\
\text { difficult. }\end{array}$ \\
\hline Chemical oxidation & $70-97$ & $47-194$ & $\begin{array}{l}\text { Removes more total CVOCs than enhanced } \\
\text { biodegradation but shows more rebound }{ }^{1} \text {. Can change } \\
\text { geochemistry of Scenario } 7 \text { site to aerobic conditions for } \\
\text { some period after treatment. Can change microbial } \\
\text { population and composition. Heterogeneous aquifer } \\
\text { conditions may make application difficult. May be more } \\
\text { suitable for anoxic or aerobic sites. }\end{array}$ \\
\hline Surfactant/cosolvents & $92-98$ & $118-1322$ & $\begin{array}{l}\text { High treatment efficiency but much higher cost. Costs } \\
\text { reflect some expensive pilot-scale projects. } \\
\text { Heterogeneous aquifer conditions may make application } \\
\text { difficult. }\end{array}$ \\
\hline Air sparging & - & - & $\begin{array}{l}\text { Not recommended at most sites. Addition of oxygen can } \\
\text { disrupt anaerobic processes. Heterogeneous aquifer } \\
\text { conditions may make application difficult. }\end{array}$ \\
\hline $\begin{array}{l}\text { Pump and Treat source } \\
\text { containment }\end{array}$ & NA & & $\begin{array}{l}\text { This approach does not reduce mass significantly } \\
\text { compared to the rate of mass loss without P\&T and may } \\
\text { need to be operated for a long time. }\end{array}$ \\
\hline
\end{tabular}




\begin{tabular}{|c|c|c|c|c|}
\hline $\begin{array}{c}\text { Simple } \\
\text { Fast }\end{array}$ & $\begin{array}{c}\text { Simple } \\
\text { Slow }\end{array}$ & $\begin{array}{c}\text { Heterogeneous } \\
\text { Fast }\end{array}$ & $\begin{array}{c}\text { Heterogeneous } \\
\text { Slow }\end{array}$ & $\begin{array}{c}\text { Fractured/ } \\
\text { Porous Rock }\end{array}$ \\
\hline
\end{tabular}

\section{SCENARIO NUMBER 8}

Faster Flow With Significant Heterogeneities and Anoxic
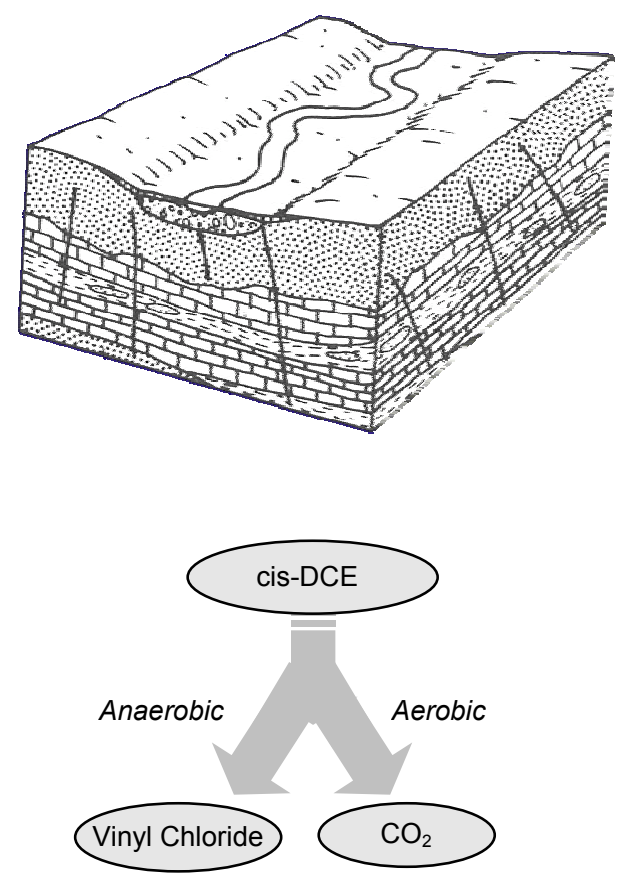


\begin{tabular}{|c|c|c|c|c|}
\hline $\begin{array}{c}\text { Simple } \\
\text { Fast }\end{array}$ & $\begin{array}{c}\text { Simple } \\
\text { Slow }\end{array}$ & $\begin{array}{c}\text { Heterogeneous } \\
\text { Fast }\end{array}$ & $\begin{array}{c}\text { Heterogeneous } \\
\text { Slow }\end{array}$ & $\begin{array}{c}\text { Fractured/ } \\
\text { Porous Rock }\end{array}$ \\
\hline
\end{tabular}

GEOCHEMICAL SETTING

\section{SCENARIO 8 DESCRIPTION: FAST FLOW WITH SIGNIFICANT HETEROGENEITIES and ANOXIC}

The hydrogeologic setting and geochemical setting below define the basic scenario type. This scenario has the following characteristics:

\section{Hydrogeologic Setting}

"Faster Flow With Significant Heterogeneities" Hydrogeology:

- Potentially multiple hydrogeologic units

- Wide distribution of hydraulic conductivity

- $\quad$ Relatively high groundwater seepage velocity

(see Section 2.1 and Appendix 1 for more information)

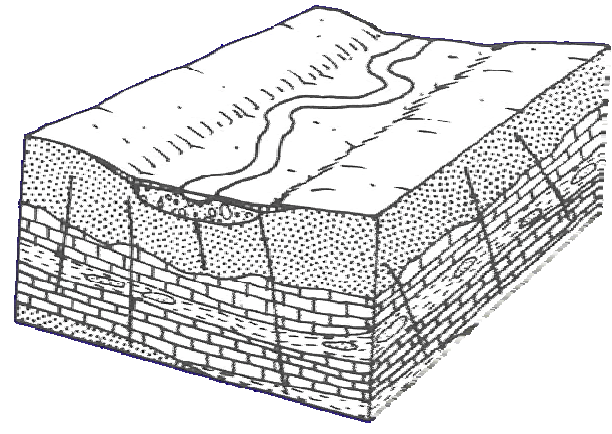

Block Diagram of River Alluvium with Overbank Deposits Aquifer from DRASTIC System

\section{Geochemical Setting}

"Anoxic" Geochemistry:

- Dissolved oxygen is low, redox is medium to low

- There are no, or limited, indicators of significant activity of anaerobic bacteria

(see Section 2.2 for more information)

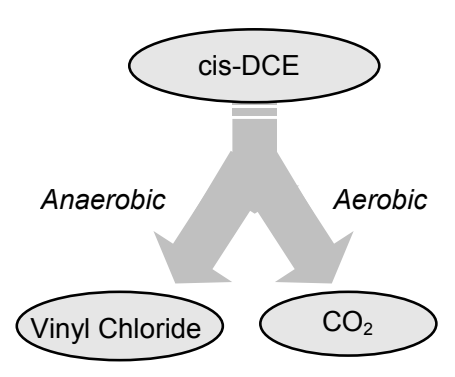

Example Reactions for "Anoxic" Geochemical Setting 


\begin{tabular}{|c|c|c|c|c|c|c|c|}
\hline $\begin{array}{c}\text { Simple } \\
\text { Fast }\end{array}$ & $\begin{array}{c}\text { Simple } \\
\text { Slow }\end{array}$ & $\begin{array}{c}\text { Heterogeneous } \\
\text { Fast }\end{array}$ & $\begin{array}{c}\text { Heterogeneous } \\
\text { Slow }\end{array}$ & $\begin{array}{c}\text { Fractured/ } \\
\text { Porous Rock }\end{array}$
\end{tabular}$\quad$\begin{tabular}{ll} 
Aerobic & Anoxic \\
\hline
\end{tabular}

\section{KEY DECHLORINATION REACTIONS}

\section{Reaction Overview}

The chart to the right shows which reactions are likely to occur, which occur but at a slow rate, which may occur under specific conditions, and which are unlikely to occur.

\section{Typically Biodegradable Parent Compounds}

These compounds may be degradable under anoxic conditions:

- PCE

- TCE

- 1,1,1-TCA

- 1,2-DCA

- $\mathrm{CT}$

\section{Typical Daughter Products}

Daughter products that may be present depending on the parent compound and the reactions listed to the right:

- TCE

- $\quad$ cis 1,2-DCE

- VC

- 1,2-DCA

- 1,1-DCE

- $\mathrm{CF}$

See Section 5.1 for more information about reactions

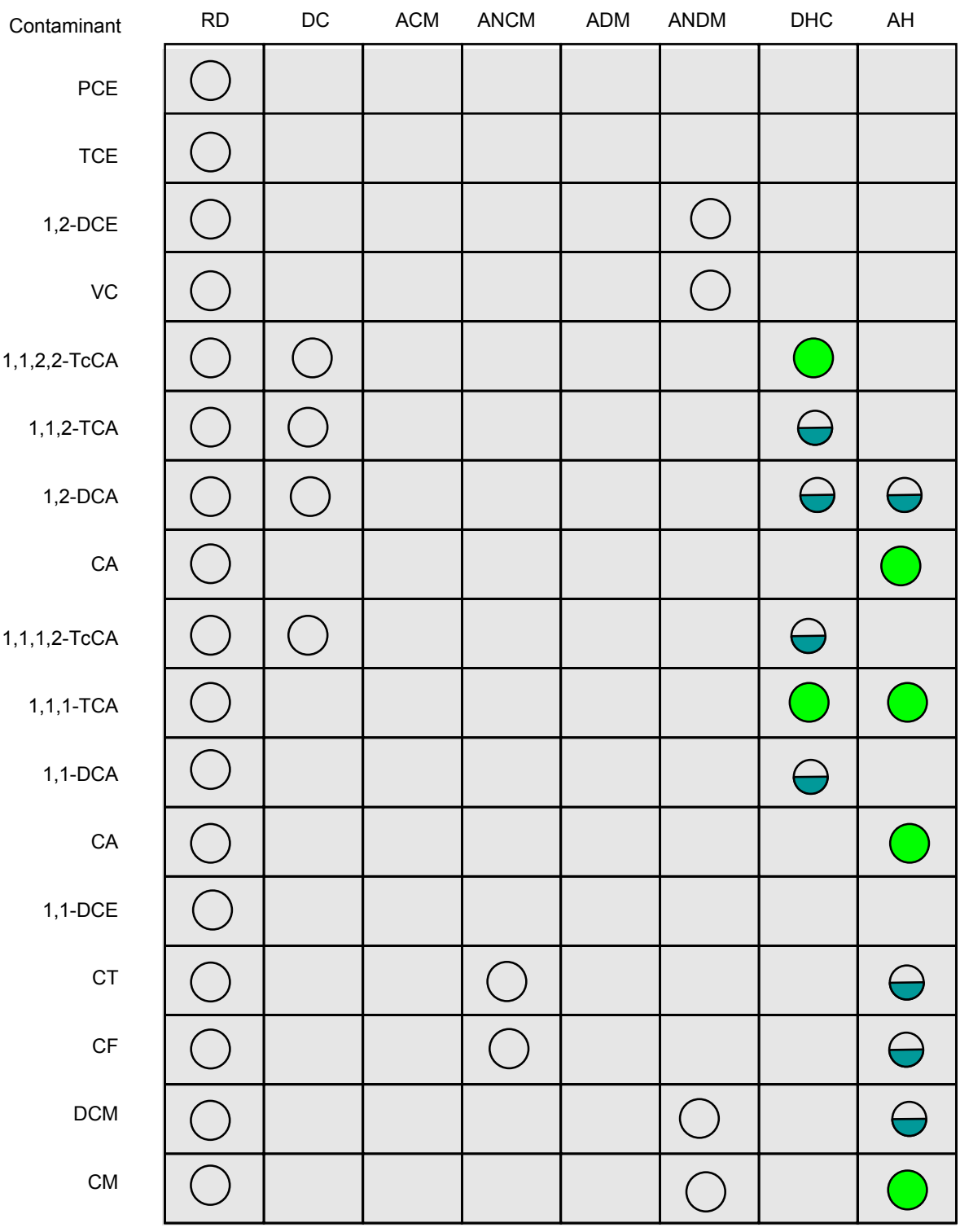

REACTIONS
Key:

Highly likely to occur, but a slow rate

May occur under specific conditions

Highly Unlikely to occur
Highly Likely to occur 


\begin{tabular}{|c|c|c|c|c|}
\hline $\begin{array}{c}\text { Simple } \\
\text { Fast }\end{array}$ & $\begin{array}{c}\text { Simple } \\
\text { Slow }\end{array}$ & $\begin{array}{c}\text { Heterogeneous } \\
\text { Fast }\end{array}$ & $\begin{array}{c}\text { Heterogeneous } \\
\text { Slow }\end{array}$ & $\begin{array}{c}\text { Fractured/ } \\
\text { Porous Rock }\end{array}$ \\
\hline
\end{tabular}

\begin{tabular}{|l|l|l|}
\hline Aerobic & Anoxic & Anaerobic \\
\hline
\end{tabular}

\section{EFFECT OF MODIFYING FACTORS}

\begin{tabular}{|c|c|}
\hline MODIFYING FACTOR & KEY POINTS \\
\hline Source Strength & $\begin{array}{l}\text { IF YOU HAVE A STRONG SOURCE: } \\
\text { - } \quad \text { Plumes (both parent and daughter compounds) may be longer } \\
\text { - } \quad \text { Source zones may persist for longer periods of time } \\
\text { - More likely to need EA or source control measures } \\
\text { IF YOU HAVE A MODERATE SOURCE: } \\
\text { - Intermediate condition between Strong and Weak Source } \\
\text { IF YOU HAVE A WEAK SOURCE: } \\
\text { - Plumes may be shorter } \\
\text { - } \quad \text { Source zones may not persist as long } \\
\text { - } \quad \text { MNA alone or MNA with EA more likely to be sufficient }\end{array}$ \\
\hline Source & $\begin{array}{l}\text { IF YOU HAVE MOSTLY A VADOSE ZONE SOURCE: } \\
\text { - Source may appear small due to dilution but can be large } \\
\text { - Sources in clay vadose zones will be weaker but more long-lived than } \\
\text { sandy vadose zone } \\
\text { - Plumes will be thinner and closer to water table } \\
\text { IF YOU HAVE A SUBMERGED SOURCE: } \\
\text { - Complex hydrogeology means matrix diffusion may be important } \\
\text { - Source mass flux can decrease relatively rapidly as DNAPL fingers } \\
\text { - } \quad \text { dissolve } \\
\text { IF YOU HAVE A MIXED SOURCE: } \\
\text { - May have combination of the factors above }\end{array}$ \\
\hline Travel Tim & $\begin{array}{l}\text { CLOSE RECEPTORS (<2 YEARS TRAVEL TIME) } \\
\text { - More intensive monitoring system likely to be needed due to potential } \\
\text { - } \\
\text { Merious consequences in event of failure of MNA/EA. } \\
\text { MODERATE RECEPTORS ( }>2 \text { but }<5 \text { YEARS TRAVEL TIME) } \\
\text { - Represents middle-ground case } \\
\text { DISTANT RECEPTORS ( }>5 \text { YEARS TRAVEL TIME) } \\
\text { - Less intensive monitoring system likely to be needed due to lower } \\
\text { - } \quad \text { MNtential for serious consequences in event of failure of MNA/EA. }\end{array}$ \\
\hline Plume Stability & $\begin{array}{l}\text { EXPANDING OR PERTURBED }{ }^{1} \text { PLUME } \\
-\quad \text { More intensive monitoring system likely to be needed } \\
\text { STABLE PLUME } \\
\text { - Represents middle-ground case } \\
\text { SHRINKING PLUME } \\
\text { - Less intensive monitoring system likely to be needed } \\
\text { - MNA alone likely to be sufficient } \\
{ }^{1} \text { For instance if the plume has been impacted by a previous remedy such as P\&T. }\end{array}$ \\
\hline
\end{tabular}




\begin{tabular}{|c|c|c|c|c|c|c|c|}
\hline $\begin{array}{c}\text { Simple } \\
\text { Fast }\end{array}$ & $\begin{array}{c}\text { Simple } \\
\text { Slow }\end{array}$ & $\begin{array}{c}\text { Heterogeneous } \\
\text { Fast }\end{array}$ & $\begin{array}{c}\text { Heterogeneous } \\
\text { Slow }\end{array}$ & $\begin{array}{c}\text { Fractured/ } \\
\text { Porous Rock }\end{array}$ \\
\hline
\end{tabular}

\section{WILL MNA WORK?}

\section{Potential for MNA Processes to Control Plume}

Because at Scenario 8 sites there are not clear indicators that the type of conditions conducive to MNA are present, it is initially uncertain whether natural attenuation processes will be suitable to manage the contaminants in the plume or plume segment. Typically, more in-depth investigation of the site attenuation processes and more rigorous monitoring are needed to evaluate the extent of natural attenuation processes and the ability of MNA to meet the remediation objectives. Some form of enhanced attenuation may be needed to couple with MNA as the remedy.

In a fast-flowing heterogeneous aquifer, relatively high rates of contaminant degradation may be needed to stabilize the plume. If reductive dechlorination processes are occurring under these conditions, it would be expected that the daughter product plume would be larger than the parent product plume because the degradation rate of daughters is typically slower than the rate for the parents.

The plume shape will be controlled by the heterogeneities in the plume segment. Plumes can be difficult to delineate, and can have complex, 3-dimensional shapes. Matrix diffusion effects in low-permeability zones can result in slowerthan-expected plume growth, which can be mistaken for mass destruction.

If the plume is shown to be either stable or shrinking then natural attenuation processes (primarily reductive dechlorination) alone have been vigorous enough to date to prevent further migration of the plume or plume segment. Under these conditions MNA may be appropriate, but it may still be difficult to identify the specific attenuation mechanism under the anoxic geochemical conditions.

At some Scenario 8 sites, "DCE stall" may be of concern and an indication that conditions are not suitable for complete dechlorination of the source contaminants. DCE stall is an informal term typically used to describe conditions at chlorinated ethene sites where the cis-1,2-DCE "stalls outs" or exhibits a very low conversion rate to VC. This DCE "stall" condition has been ascribed to a variety of factors, including:

- Lack of the necessary microbiological communities that are required to degrade cis-1,2-DCE to VC;

- The direct conversion of cis-1,2-DCE to carbon dioxide, which makes it appear that cis-1,2-DCE is not being biodegraded because VC is not being produced; but in fact the cis-1,2-DCE is being biodegraded by direct oxidation to carbon dioxide;

- Conditions which are anaerobic enough to support the conversion of TCE to cis-1,2-DCE but not anaerobic enough to support the conversion from cis-1,2-DCE to VC by reductive dechlorination;

- Toxicity effects caused at sites where sulfate reducers are producing hydrogen sulfide $\left(\mathrm{H}_{2} \mathrm{~S}\right)$, but the $\mathrm{H}_{2} \mathrm{~S}$ is not being precipitated fast enough by ferrous iron (a by-product of ferric iron reduction) to prevent toxicity effects in the cis-1,2-DCE degraders.

While the cause of cis-1,2-DCE stall is still being evaluated by a number of researchers, the main implication is that at some chlorinated ethene sites, cis-1,2-DCE plumes are expanding and not being controlled. DCE "stall" does not affect long-term sustainability of a reaction, but does determine if natural attenuation processes are sufficient to prevent migration of the plume.

\section{Key Sustainability Concept}

Fast-flowing plume segments can have high mass flux of contaminants leaving the source, and therefore high rates of degradation are often needed to attenuate the plume. Sufficient natural substrate (e.g., organic matter) or cocontaminants that serve as electron donors and can act as a substrate are needed to sustain high rates of degradation for the anaerobic reactions using the chlorinated solvent as an electron acceptor that may be occurring under the anoxic geochemical conditions. In a heterogeneous system, care should be taken to consider whether there are conduits for contaminant migration that do not have sufficient substrate available (e.g., a clean sand layer). For some contaminants under anoxic conditions, biological reactions use the chlorinated solvent as the electron donor. At Scenario 8 sites, non-biologically catalyzed attenuation processes may be the primary attenuation processes. In this case, the processes are likely sustainable, but may be difficult to identify and quantify. 


\begin{tabular}{|c|c|c|c|c|c|c|c|}
\hline $\begin{array}{c}\text { Simple } \\
\text { Fast }\end{array}$ & $\begin{array}{c}\text { Simple } \\
\text { Slow }\end{array}$ & $\begin{array}{c}\text { Heterogeneous } \\
\text { Fast }\end{array}$ & $\begin{array}{c}\text { Heterogeneous } \\
\text { Slow }\end{array}$ & $\begin{array}{c}\text { Fractured/ } \\
\text { Porous Rock }\end{array}$ \\
\hline
\end{tabular}

\section{HOW DO I CHARACTERIZE THIS TYPE OF SITE?}

\section{Actions Needed to Determine MNA Viability}

The following are key considerations for evaluating the viability of MNA at a Fast Flow With Significant Heterogeneities and Anoxic type site:

- $\quad$ assess the site geochemical, hydraulic, and contaminant conditions in detail to assess the type and extent/rate of attenuation processes - this assessment may require significant effort depending on the site conditions, however, the uniform hydraulic conditions will help simplify some parts of the assessment;

- $\quad$ determine the relative horizontal and vertical plume movement and whether there are layers (e.g., sandy units) where the plume movement is significantly greater than in other parts of the aquifer;

- $\quad$ both parent compound and daughter compounds need to be delineated (the anoxic setting means that a number of daughter products may be generated);

- $\quad$ determine if plume(s) are expanding/perturbed, stable, or shrinking;

- determine the location of any receptors (if present) and determine the travel time from the edge of the plume to these receptors;

- a mass-balance type evaluation of the source zone will help to determine if the electron donor supply is sustainable over the long term if the attenuation reactions are determined to be primarily anaerobic dechlorination with the contaminant acting as the electron acceptor.

\section{Key Monitoring Concepts}

The fast hydrogeologic setting may mean the plume can be relatively large, and therefore require more monitoring points. In addition, the plume can become stable (if it is going to) more quickly than for a slow hydrogeologic setting, so an extremely long temporal record (i.e., the number of years of monitoring data you have) may not be needed to determine plume stability.

A more extensive monitoring system will likely be required to delineate the plume, because heterogeneities can result in wide distribution of hydraulic conductivity across the plume segment. Attention to vertical characteristics of the plume and monitoring within specific hydrologic layers may be particularly important. Plumes can have unusual shapes, such as apparent cross-gradient (regional gradient) flow patterns. This type of hydrogeologic setting benefits from plume delineation strategies using direct push approaches and adaptive plume delineation strategies.

\section{Key Uncertainty Concepts}

A key uncertainty for this scenario is associated with knowing that the plume is sufficiently delineated. In a heterogeneous subsurface, appropriate selection of monitoring locations is more difficult both horizontally and vertically within the plume. Because all of the MNA evaluation methods rely on a suitable conceptual model and data to describe the plume and subsurface properties, more data is typically required in a heterogeneous system to complete the evaluation at a level of detail acceptable to decision makers. Another uncertainty may be the sustainability of MNA due to the high mass flux of the source that may be present (see key sustainability concept section). There may be less uncertainty about the plume conditions in general under this scenario if the plume appears to be fully developed and it is evident from a short duration of contaminant monitoring that MNA is currently working. However, the anoxic geochemical setting may cause considerable uncertainty in evaluating MNA because it may be more difficult to identify and quantify the attenuation processes. 


\begin{tabular}{|c|c|c|c|c|}
\hline $\begin{array}{c}\text { Simple } \\
\text { Fast }\end{array}$ & $\begin{array}{c}\text { Simple } \\
\text { Slow }\end{array}$ & $\begin{array}{c}\text { Heterogeneous } \\
\text { Fast }\end{array}$ & $\begin{array}{c}\text { Heterogeneous } \\
\text { Slow }\end{array}$ & $\begin{array}{c}\text { Fractured/ } \\
\text { Porous Rock }\end{array}$ \\
\hline
\end{tabular}

\begin{tabular}{|l|c|c|}
\hline \multicolumn{3}{|c}{ GEOCHEMICAL SETTING } \\
\hline Aerobic & Anoxic & Anaerobic \\
\hline
\end{tabular}

\section{HOW DO I ANALYZE DATA?}

In a fast-flowing aquifer, a plume is more likely to show concentration differences over a longer distance and the plume will become stable (if it is going to) in a shorter period of time than in slower-flow aquifers. A good first step in this type of aquifer is to examine plume maps, concentration vs. time at each well, and concentration vs. distance plots to determine whether the plume is attenuating. However, care must be taken to ensure that the monitoring network is sufficient for the heterogeneous conditions at the site. Concentrations may not show a progression of parent to daughter products with distance. Thus, it is likely that contaminant monitoring over a period of time will be needed to establish trends in the plume size and concentration data. In some cases, this type of data will be sufficient for a fast-flowing aquifer with anoxic conditions.

Transport models can be very helpful in analyzing and visualizing the data and expected plume conditions for comparison to field data. Transport models may be needed to predict the future state of the plume so that decisions can be made in a timely fashion and then confirmed through the long-term monitoring portion of MNA implementation. A simple transport model such as BIOCHLOR can be helpful as a screening tool in analyzing and visualizing the data and expected plume conditions for comparison to field data. However, BIOCHLOR will not be sufficient to describe the range of attenuation processes that may be important under anoxic geochemical conditions and may not be sufficient to describe the flow conditions in a heterogeneous aquifer. As such, more complex numerical modeling is more likely to be needed as part of MNA evaluation for Scenario 8 sites. To support this more complex analysis, microcosm tests, molecular probes, and more detailed field measurements may be required. The chart below summarizes an approach for analyzing data at sites depending on whether the concentration data indicates that the plume is decreasing, stable, or increasing and the source type. As noted in the table, as the source gets stronger and the plume is less likely to be decreasing in extent, more information is needed to support an MNA remedy.

\begin{tabular}{|c|c|c|c|}
\hline & \multicolumn{3}{|c|}{ PLUME STATUS } \\
\hline $\begin{array}{l}\text { CONTAMINANT } \\
\text { CONCENTRATIONS/GEOCHEMICAL } \\
\text { STATUS }\end{array}$ & $\begin{array}{l}\text { DECREASING OR } \\
\text { PROBABLY } \\
\text { DECREASING }\end{array}$ & STABLE & $\begin{array}{l}\text { INCREASING, } \\
\text { PROBABLY } \\
\text { INCREASING, OR } \\
\text { PERTURBED }^{1}\end{array}$ \\
\hline Weak Source & - Mass loss & $\begin{array}{l}\text { - } \text { Mass loss } \\
\text { - } \text { Geochemical footprints } \\
\text { Simple Model/Special } \\
\text { Studies }\end{array}$ & $\begin{array}{l}\text { - Mass loss } \\
\text { - Geochemical footprints } \\
\text { - Comprehensive Model/ } \\
\text { Special Studies }\end{array}$ \\
\hline Strong Source & $\begin{array}{ll}\text { - } & \text { Mass loss } \\
\text { - } & \text { Geochemical footprints } \\
\text { - } & \text { Simple Model }\end{array}$ & $\begin{array}{l}\text { - } \text { Mass loss } \\
\text { - } \text { Coochemical footprints } \\
\text { Special Studies }\end{array}$ & $\begin{array}{l}\text { - Mass loss } \\
\text { - Geochemical footprints } \\
\text { - Comprehensive Model/ } \\
\text { Special Studies }\end{array}$ \\
\hline
\end{tabular}

${ }^{1}$ For instance if the plume has been impacted by a previous remedy such as P\&T.

To demonstrate mass loss construct these graphics:

- Concentration vs. time plots at individual wells;

- Concentration vs. distance plots, with multiple lines for different sampling events through time;

- Plume maps showing plume extent at different times (i.e., either panel maps, or one map with several plume boundaries for different times).

To show geochemical footprints make tables or figures that show:

- Daughter product production;

- Ethene/Ethane production;

- Chloride production (this may not work at many sites, however, due to background chloride);

- Low dissolved oxygen (shows anoxic geochemical conditions);

- Methane and iron(II) distribution (indicators of anaerobic activity);

- Nitrate and sulfate distribution (indicators of competing electron acceptors);

To perform modeling, typical tools include the following:

- Simple transport model (analytical model, e.g., BIOCHLOR, BIOBALANCE ${ }^{1}$ );

- Comprehensive transport model (numerical model, e.g., RT3D). 


\begin{tabular}{|c|c|c|c|c|c|c|c|}
\hline $\begin{array}{c}\text { Simple } \\
\text { Fast }\end{array}$ & $\begin{array}{c}\text { Simple } \\
\text { Slow }\end{array}$ & $\begin{array}{c}\text { Heterogeneous } \\
\text { Fast }\end{array}$ & $\begin{array}{c}\text { Heterogeneous } \\
\text { Slow }\end{array}$ & $\begin{array}{c}\text { Fractured/ } \\
\text { Porous Rock }\end{array}$ \\
\hline
\end{tabular}

If a special study is needed, some of the following may be applicable:

- Carbon/chlorine isotope analysis (indicator of degradation processes)

- Molecular probes (indicators of microbial activity when other geochemical indicators are ambiguous);

- Microcosm tests (determine the reaction processes occurring at the site).

1 Developed by Groundwater Services (www.gsi-net.com), with the support of the Savannah River National Laboratory and DOE, to evaluate monitored natural attenuation at chlorinated solvent sites.

\section{WHAT ABOUT COSTS AND ENHANCEMENTS?}

Costs for evaluating and implementing MNA for this scenario are primarily dependent on the following items.

- $\quad$ Source strength - Stronger and longer lasting sources will be more costly especially due to the high groundwater flow rate.

- $\quad$ Depth to the plume and size of the plume - Deeper, larger plumes require more and costlier monitoring wells.

- Travel time to the receptor - Plumes closer to receptors will require more frequent monitoring especially with the high groundwater flow rate.

- $\quad$ Plume stability - Less stable plumes require more rigorous evaluation and monitoring to select and implement MNA especially with the high groundwater flow rate.

- Extent of variability in geochemical conditions - More variability will likely require more characterization and monitoring to assess attenuation conditions within each different geochemical zone.

- $\quad$ Nature of Heterogeneities - More heterogeneous aquifers may require a larger number of monitoring locations and more detailed analysis of flow and transport as part of evaluating MNA.

MNA may be a viable single remedy for the site. If it is determined that MNA may not meet remediation goals, a good first option is to evaluate the potential use of sustainable enhancements (enhanced attenuation). The objective being to adjust the attenuation conditions sufficiently such that the plume is controlled. If the enhancements are insufficient to control the plume, source control treatment may be required. In general, enhanced attenuation is less likely a viable option for a fast flow regime with a strong source, unless source treatment is undertaken initially. The sections below discuss options for enhanced attenuation and source control related to this scenario.

\section{Key Enhanced Attenuation Concepts}

Enhanced attenuation must be carefully designed in a heterogeneous flow system. Application of enhancements may be difficult due to the fast groundwater flow conditions. Enhancements are presented organized by the different zones in which they may be applied: source zone (reduction of contaminant mass flux to plume); plume (enhanced attenuation processes); or discharge zone (enhanced attenuation processes). Within the source zone enhancements can be applied as a hydraulic manipulation or as a passive source reduction (active source control is discussed in the next section). Within the plume and discharge zone, either biological (microbial or plant based) or abiotic (abiotic degradation, reactive barriers, sorption) attenuation processes can be enhanced. A description of potential enhanced attenuation approaches, and their applicability to Scenario 8 sites, is shown below. More detailed information about each technology listed below is available in Early et al., (2005). 


\begin{tabular}{|c|c|c|c|c|}
\hline $\begin{array}{c}\text { Simple } \\
\text { Fast }\end{array}$ & $\begin{array}{c}\text { Simple } \\
\text { Slow }\end{array}$ & $\begin{array}{c}\text { Heterogeneous } \\
\text { Fast }\end{array}$ & $\begin{array}{c}\text { Heterogeneous } \\
\text { Slow }\end{array}$ & $\begin{array}{c}\text { Fractured/ } \\
\text { Porous Rock }\end{array}$ \\
\hline
\end{tabular}

\begin{tabular}{|l|l|l|}
\hline Aerobic & Anoxic & Anaerobic \\
\hline
\end{tabular}

\section{Enhancement Summary}

\begin{tabular}{l} 
Enhancement \\
Source Zone Enhancement \\
$\begin{array}{l}\text { Surface water or } \\
\text { groundwater } \\
\text { interception/diversion }\end{array}$ \\
\hline Physical containment \\
\hline Passive extraction \\
\hline Enhanced biodegradation \\
\hline Permeable reactive \\
barrier
\end{tabular}

Plume and Discharge Zone Enhancements

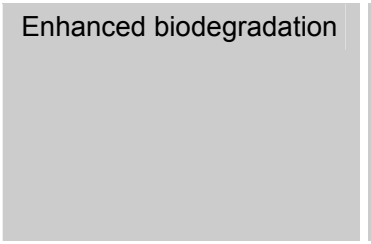

Permeable reactive barrier

\section{Description}

Use of interception trenches or wells, surface covers, or phyto-covers (plants) to reduce water flux through source area and/or divert unwanted electron acceptors.

Use of grout walls and other physical containment

Use of passive soil vapor extraction

Injection of electron donor (e.g., HRC, molasses, vegetable oil) to enhance microbial degradation of the source.

Use of zero valent iron, reduced sediment iron, enhanced partitioning, or biological barrier to attenuate some of the contaminant flux at the downgradient edge of the source area.
May be more difficult in high flow rate conditions. May be difficult in heterogeneous conditions. Likely more applicable using surface covers or phyto-covers if the source is primarily within the vadose zone.

Potentially applicable depending on the geometry of the source zone.

Useful if the source is primarily within the vadose zone.

Potentially well suited for anoxic sites because oxygen concentrations are already low and anaerobic processes may be readily stimulated. Complex hydrogeology can make application difficult. Faster groundwater flow may require larger amounts of electron donor than in slower groundwater if electron acceptors are carried into the treatment zone.

May be more difficult and expensive to construct due to heterogeneous conditions. May be more difficult and expensive to construct due to fast flow regime. Faster groundwater flows potentially result in more contaminant and competing electron acceptors passing through the barrier, requiring greater thickness to achieve desired treatment levels. Anoxic conditions are helpful because oxygen concentration is low and will not disrupt barriers using anaerobic reactions.
Potentially well suited for anoxic sites because oxygen concentrations are already low and anaerobic processes may be readily stimulated. Complex hydrogeology can make application difficult. Faster groundwater flow may require larger amounts of electron donor than in slower groundwater if electron acceptors are carried into the treatment zone.

Use of zero valent iron, reduced sediment iron, enhanced partitioning, or biological barrier to attenuate some of the contaminant flux within the plume.

Phytoextraction

Plant-based hydraulic control (plume enhancement only)

Injection of electron molasses, vegetable oil) to enhance microbial degradation of the source.

May be more difficult and expensive to construct due to heterogeneous conditions. May be more difficult and expensive to construct due to fast flow regime. Faster groundwater flows potentially result in more contaminant and competing electron acceptors passing through the barrier, requiring greater thickness to achieve desired treatment levels. Anoxic conditions are helpful because oxygen concentration is low and will not disrupt barriers using anaerobic reactions.

Use of plants to extract contaminants from near surface groundwater

Use of plants to control hydraulic gradient and slow groundwater
Heterogeneous aquifer conditions may make application difficult. May be less effective for fast groundwater flow conditions.

Heterogeneous aquifer conditions may make application difficult. May be less effective for fast groundwater flow conditions. 


\begin{tabular}{|c|c|c|c|c|}
\hline $\begin{array}{c}\text { Simple } \\
\text { Fast }\end{array}$ & $\begin{array}{c}\text { Simple } \\
\text { Slow }\end{array}$ & $\begin{array}{c}\text { Heterogeneous } \\
\text { Fast }\end{array}$ & $\begin{array}{c}\text { Heterogeneous } \\
\text { Slow }\end{array}$ & $\begin{array}{c}\text { Fractured/ } \\
\text { Porous Rock }\end{array}$ \\
\hline
\end{tabular}

\begin{tabular}{|l|c|c|}
\hline \multicolumn{3}{|c}{ GEOCHEMICAL SETTING } \\
\hline Aerobic & Anoxic & Anaerobic \\
\hline
\end{tabular}

\section{Key Source Control Concepts}

A description of potential source control measures, and their applicability to Scenario 8 sites, is shown below. Note that source control measures are unlikely to achieve complete restoration at a site, and some source material is always left behind after treatment (U.S. EPA, 2003; McGuire et al., 2006).

\begin{tabular}{|c|c|c|c|}
\hline$\frac{\text { Example }}{\text { Technology }}$ & $\begin{array}{l}\text { Performance } \\
\left(25^{\text {th }}-75^{\text {th }} \text { Percentile }\right. \\
\% \text { reduction in } \\
\text { parent compound })^{1}\end{array}$ & $\begin{array}{l}\frac{\text { Unit Cost }}{25^{\text {th }}-75^{\text {th }}} \\
\text { Percentile } \\
\text { (\$/yrd) })^{2}\end{array}$ & Applicability to Scenario 8 Sites \\
\hline In-situ biodegradation & $73-99$ & $27-152$ & $\begin{array}{l}\text { Potentially well suited for anoxic sites because } \\
\text { oxygen concentrations are already low and } \\
\text { anaerobic processes may be readily stimulated. } \\
\text { Complex hydrogeology can make application } \\
\text { difficult. Faster groundwater flow may require } \\
\text { larger amounts of electron donor than in slower } \\
\text { groundwater if electron acceptors are carried into } \\
\text { the treatment zone. Least expensive treatment } \\
\text { option. }\end{array}$ \\
\hline Thermal treatment & $68-99.9$ & $48-129$ & $\begin{array}{l}\text { Does not appear to disrupt MNA after treatment. } \\
\text { Heterogeneous aquifer conditions may make } \\
\text { application difficult. }\end{array}$ \\
\hline Chemical oxidation & $70-97$ & $47-194$ & $\begin{array}{l}\text { Removes more total CVOCs than enhanced } \\
\text { biodegradation but shows more rebound }{ }^{1} \text {. Can } \\
\text { change geochemistry of Scenario } 8 \text { site to aerobic } \\
\text { conditions for some period after treatment. Can } \\
\text { change microbial population and composition. } \\
\text { Heterogeneous aquifer conditions may make } \\
\text { application difficult. }\end{array}$ \\
\hline Surfactant/cosolvents & $92-98$ & $118-1322$ & $\begin{array}{l}\text { High treatment efficiency but much higher cost. } \\
\text { Costs reflect some expensive pilot-scale projects. } \\
\text { Heterogeneous aquifer conditions may make } \\
\text { application difficult. Some surfactants/cosolvents } \\
\text { can serve as electron donors for subsequent } \\
\text { anaerobic biodegradation reactions. }\end{array}$ \\
\hline Air sparging & - & - & $\begin{array}{l}\text { May be suitable if only limited biological } \\
\text { attenuation is occurring at a site. Addition of } \\
\text { oxygen can disrupt anaerobic processes that may } \\
\text { be occurring. Heterogeneous aquifer conditions } \\
\text { may make application difficult. }\end{array}$ \\
\hline $\begin{array}{l}\text { Pump and Treat } \\
\text { source containment }\end{array}$ & NA & & $\begin{array}{l}\text { Due to the high groundwater flow rate, a large } \\
\text { system may be required. This approach does not } \\
\text { reduce mass significantly compared to the rate of } \\
\text { mass loss without P\&T and may need to be } \\
\text { operated for a long time. }\end{array}$ \\
\hline
\end{tabular}

${ }^{1}$ McGuire et al., 2006. ${ }^{2}$ McDade et al., 2005. 


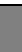

\section{SCENARIO NUMBER 9}

Faster Flow With

Significant

Heterogeneities

and

Aerobic
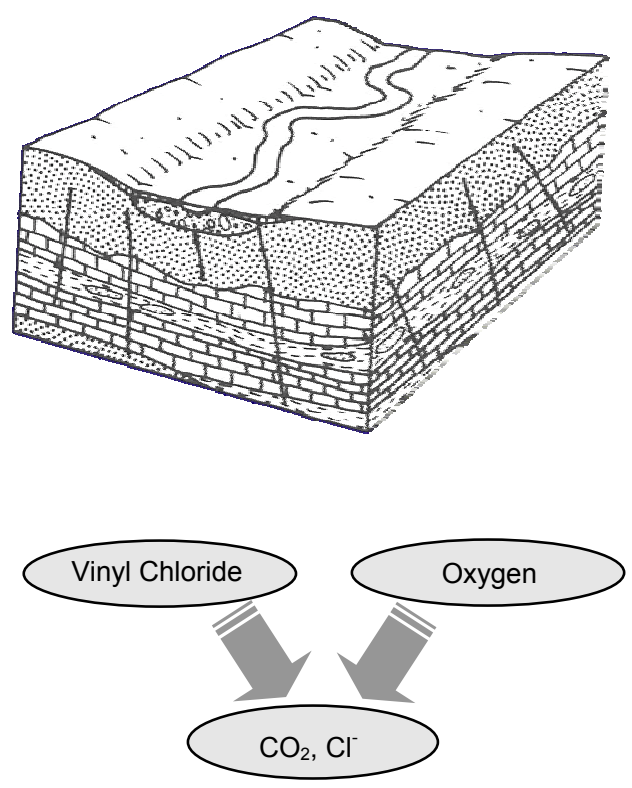


\section{SCENARIO 9 DESCRIPTION: FAST FLOW WITH SIGNIFICANT HETEROGENEITIES and AEROBIC}

The hydrogeologic setting and geochemical setting below define the basic scenario type. This scenario has the following characteristics:

\section{Hydrogeologic Setting}

"Faster Flow With Significant Heterogeneities" Hydrogeology:

- Potentially multiple hydrogeologic units

- Wide distribution of hydraulic conductivity

- Relatively high groundwater seepage velocity

(see Section 2.1 and Appendix 1 for more information)

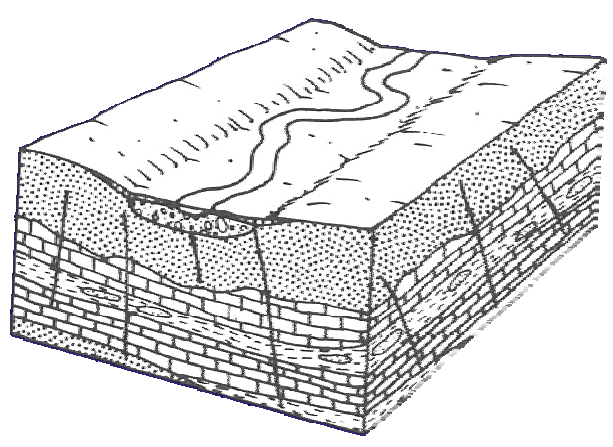

Block Diagram of River Alluvium with Overbank Deposits Aquifer from DRASTIC System

\section{Geochemical Setting}

"Aerobic" Geochemistry:

- Dissolved oxygen and redox are moderate to high

- Possible to have wide range of concentrations of competing electron acceptors (nitrate, sulfate)

- No or very limited presence of anaerobic indicators (e.g., methane).

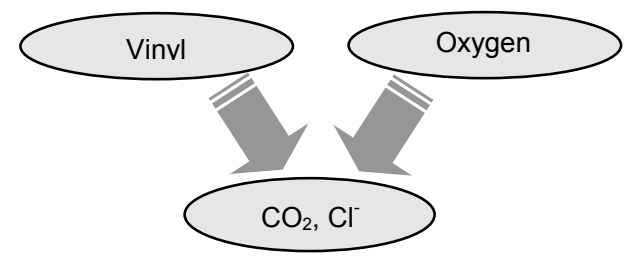

Example Reactions for "Aerobic" Geochemical Setting 


\section{KEY DECHLORINATION REACTIONS}

\section{Reaction Overview}

The chart to the right shows which reactions are likely to occur, which occur but at a slow rate, and which may occur under specific conditions, and which are unlikely to occur.

\section{Compounds Easier for Biological Degradation}

- $\quad$ cis 1,2-DCE

- $\mathrm{VC}$

- 1,2-DCA

- 1,1-DCE

- DCM

- $\mathrm{CM}$

\section{Compounds More Difficult for Biological Degradation}

- PCE

- TCE

- $\mathrm{CT}$

- $\mathrm{CF}$

See Section 5.1 for more information about reactions

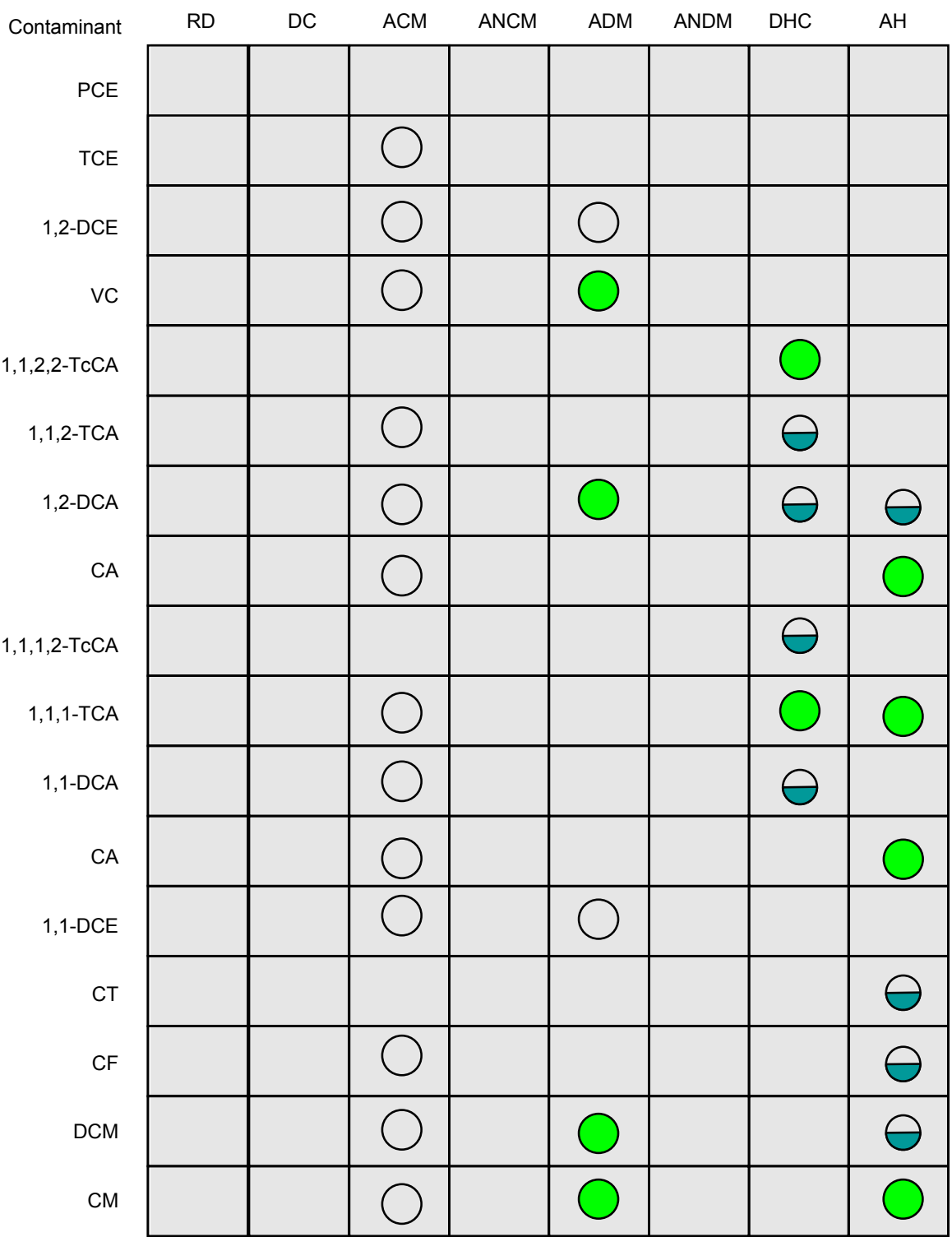

REACTIONS

Key:

Highly Likely to occur

$\bigoplus$ Highly likely to occur, but a slow rate

$\bigcirc$ May occur under specific conditions

Highly Unlikely to occur

\begin{tabular}{|c|l|}
\hline ACM & Aerobic Co-Metabolism \\
\hline ANCM & Anaerobic Co-Metabolism \\
\hline ADM & Aerobic Direct Metabolism \\
\hline ANDM & Anaerobic Direct Metabolism \\
\hline DHC & Dehydrochlorination (abiotic) \\
\hline AH & Abiotic Hydrolysis \\
\hline DC & Dichloroelimination (biotic) \\
\hline RD & Reductive Dechlorination (hydrogenolysis) \\
\hline
\end{tabular}




\section{EFFECT OF MODIFYING FACTORS}

\begin{tabular}{|c|c|}
\hline MODIFYING FACTOR & KEY POINTS \\
\hline Source Strength & $\begin{array}{l}\text { IF YOU HAVE A STRONG SOURCE: } \\
\text { - Plumes (both parent and daughter compounds) may be longer } \\
\text { - } \quad \text { Source zones may persist for longer periods of time } \\
\text { - More likely to need EA or source control measures } \\
\text { IF YOU HAVE A MODERATE SOURCE: } \\
\text { - Intermediate condition between Strong and Weak Source } \\
\text { IF YOU HAVE A WEAK SOURCE: } \\
\text { - Plumes may be shorter } \\
\text { - Source zones may not persist as long } \\
\text { - MNA alone or MNA with EA more likely to be sufficient }\end{array}$ \\
\hline Source & $\begin{array}{l}\text { IF YOU HAVE MOSTLY A VADOSE ZONE SOURCE: } \\
\text { - Source may appear small due to dilution but can be large } \\
\text { - } \quad \text { Sources in clay vadose zones will be weaker but more long-lived than } \\
\text { sandy vadose zone } \\
\text { - } \quad \text { Plumes will be thinner and closer to water table } \\
\text { IF YOU HAVE A SUBMERGED SOURCE: } \\
\text { - Complex hydrogeology means matrix diffusion may be important } \\
\text { - Source mass flux can decrease relatively rapidly as DNAPL fingers } \\
\text { - } \quad \text { dissolve } \\
\text { IF YOU HAVE A MIXED SOURCE: } \\
\text { - May have combination of the factors above }\end{array}$ \\
\hline Travel Time to $R$ & $\begin{array}{l}\text { CLOSE RECEPTORS (<2 YEARS TRAVEL TIME) } \\
\text { - More intensive monitoring system likely to be needed due to potential } \\
\text { - } \\
\text { Merious consequences in event of failure of MNA/EA. } \\
\text { MODERATE RECEPTORS ( }>2 \text { but }<5 \text { YEARS TRAVEL TIME) } \\
\text { - Represents middle-ground case } \\
\text { DISTANT RECEPTORS ( }>5 \text { YEARS TRAVEL TIME) } \\
\text { - Less intensive monitoring system likely to be needed due to lower } \\
\text { - } \text { potential for serious consequences in event of failure of MNA/EA. }\end{array}$ \\
\hline Plume Stability & $\begin{array}{l}\text { EXPANDING OR PERTURBED }{ }^{1} \text { PLUME } \\
-\quad \text { More intensive monitoring system likely to be needed } \\
\text { STABLE PLUME } \\
\text { - Represents middle-ground case } \\
\text { SHRINKING PLUME } \\
\text { - Less intensive monitoring system likely to be needed } \\
\text { MNA alone likely to be sufficient } \\
{ }^{1} \text { For instance if the plume has been impacted by a previous remedy such as P\&T. }\end{array}$ \\
\hline
\end{tabular}




\begin{tabular}{|c|c|c|c|c|c|c|}
\hline $\begin{array}{c}\text { Simple } \\
\text { Fast }\end{array}$ & $\begin{array}{c}\text { Simple } \\
\text { Slow }\end{array}$ & $\begin{array}{c}\text { Heterogeneous } \\
\text { Slow }\end{array}$ & $\begin{array}{c}\text { Fractured/ } \\
\text { Porous Rock }\end{array}$ \\
\hline
\end{tabular}

\section{WILL MNA WORK?}

\section{Potential for MNA Processes to Control Plume}

In fast-flowing heterogeneous plumes or plume segments where aerobic conditions are present uniformly throughout the plume, there is less likelihood that natural attenuation processes will result in short, stable or shrinking plumes than in anaerobic plumes or plume segments if parent compounds such as PCE and TCE are present. While TCE and some other parent compounds can be degraded biologically under aerobic conditions, these reactions are co-metabolic reactions that require the presence of methane or another similar substrate that are typically not present in aerobic aquifers under natural conditions. Abiotic degradation processes will occur for some compounds, but may produce daughter products that cannot be readily degraded under aerobic conditions. Some compounds can be degraded directly by aerobic bacteria (e.g., DCE and VC). In summary, aerobic conditions are generally less conducive for managing chlorinated solvent plumes, except for a plume segment downgradient of an anaerobic plume segment where the contamination is dominated by reductive dechlorination daughter products such as cis-1,2-DCE or VC that can be directly degraded under aerobic conditions.

The fast nature of the hydrogeologic setting means that: i) there will be a high mass flux of oxygen entering the plume segment, so it is less likely that direct biodegradation reactions will be oxygen-limited; and ii) it is more likely that relatively long contaminant plumes will result for compounds which do not degrade readily in aerobic geochemical settings.

The plume shape will be controlled by the heterogeneities in the plume segment. Plumes can be difficult to delineate, and can have complex, 3-dimensional shapes. Matrix diffusion effects in low-permeability zones can result in slower-than-expected plume growth, which can be mistaken for mass destruction.

\section{Key Sustainability Concept}

Direct aerobic biologic reactions and abiotic reactions are likely to be sustainable indefinitely.

Other biodegradation reactions that can occur under aerobic conditions are co-metabolic reactions that require oxygen and a primary substrate (such as methane). The probability that the supply of dissolved oxygen to the plume from upgradient sources (and plume re-aeration to a lesser degree) will be interrupted is relatively low. In a heterogeneous system, care should be taken to consider whether there are conduits for contaminant migration that do not have sufficient oxygen available. Additionally, changes in source structure over time could result in reduced delivery of the primary substrate, increasing the uncertainty in the long-term sustainability of a naturally occurring co-metabolic reaction. 


|

\begin{tabular}{|c|c|c|}
\hline $\begin{array}{c}\text { Heterogeneous } \\
\text { Slow }\end{array}$ & $\begin{array}{c}\text { Fractured/ } \\
\text { Porous Rock }\end{array}$ \\
\hline
\end{tabular}

\begin{tabular}{|l|l|l|}
\hline Aerobic & Anoxic & Anaerobic \\
\hline
\end{tabular}

\section{HOW DO I CHARACTERIZE THIS TYPE OF SITE?}

\section{Actions Needed to Determine MNA Viability:}

The following are key considerations for evaluating the viability of MNA at a Fast Flow With Significant Heterogeneities and Aerobic type site:

- $\quad$ trends for contaminant concentrations need to be established to assess whether attenuation is occurring (the aerobic setting means that daughter products will likely not be available to assess whether attenuation processes are occurring);

- determine the relative horizontal and vertical plume movement and whether there are layers (e.g., sandy units) where the plume movement is significantly greater than in other parts of the aquifer;

- confirm that aerobic conditions are present throughout the entire plume/plume segment;

- $\quad$ determine if plume(s) are expanding/perturbed, stable, or shrinking;

- determine the location of any receptors (if present) and determine the travel time from the edge of the plume to these receptors.

\section{Key Monitoring Concepts}

The fast hydrogeologic setting may mean the plume can be relatively large, and therefore require more monitoring points. In addition, the plume can become stable (if it is going to) more quickly than for a slow hydrogeologic setting, so an extremely long temporal record (i.e., the number of years of monitoring data you have) may not be needed to determine plume stability.

A more extensive monitoring system will likely be required to delineate the plume, because heterogeneities can result in wide distribution of hydraulic conductivity across the plume segment. Attention to vertical characteristics of the plume and monitoring within specific hydrologic layers may be particularly important. Plumes can have unusual shapes, such as apparent cross-gradient (regional gradient) flow patterns. This type of hydrogeologic setting benefits from plume delineation strategies using direct push approaches and adaptive plume delineation strategies.

\section{Key Uncertainty Concepts}

A key uncertainty for this scenario is associated with knowing that the plume is sufficiently delineated. In a heterogeneous subsurface, appropriate selection of monitoring locations is more difficult both horizontally and vertically within the plume. Because all of the MNA evaluation methods rely on a suitable conceptual model and data to describe the plume and subsurface properties, more data is typically required in a heterogeneous system to complete the evaluation at a level of detail acceptable to decision makers.

Additionally, because daughter compounds for direct aerobic metabolism of contaminants are not available, it may be difficult to show that this type of attenuation process is occurring.

It may also be uncertain whether co-metabolic reactions are occurring in the plume segment. To resolve this uncertainty, it may be necessary to perform a detailed analysis of contaminant loss down the centerline of the plume: i) to determine if the observed reduction in concentrations is due to dispersion only or due to a combination of dispersion and co-metabolic reactions; and ii) to determine if a primary substrate (e.g., phenol, methane, propane, etc.) is present in the plume segment. 


\begin{tabular}{c|c|c|c|c}
$\begin{array}{c}\text { Simple } \\
\text { Fast }\end{array}$ & $\begin{array}{c}\text { Simple } \\
\text { Slow }\end{array}$ & $\begin{array}{c}\text { Heterogeneous } \\
\text { Slow }\end{array}$ & $\begin{array}{c}\text { Fractured/ } \\
\text { Porous Rock }\end{array}$ \\
\hline
\end{tabular}

\begin{tabular}{|l|l|l|}
\hline Aerobic & Anoxic & Anaerobic \\
\hline
\end{tabular}

\section{HOW DO I ANALYZE DATA?}

In a fast-flowing aquifer, a plume is more likely to show concentration differences over a longer distance and the plume will become stable (if it is going to) in a shorter period of time than in slower-flow aquifers. Thus, a good first step in this type of aquifer is to examine plume maps, concentration vs. time at each well, and concentration vs. distance plots to determine whether the plume is attenuating. Concentrations should show a reduction of contaminant concentrations with distance if attenuation is occurring. However, care must be taken to ensure that the monitoring network is sufficient for the heterogeneous conditions at the site. Because daughter products are not readily measured for aerobic reactions, additional information to confirm attenuation processes may be needed. Especially if the plume edge is close to receptors, it may be necessary to provide additional data to verify aerobic degradation processes are occurring and to assess the sustainability of these processes. Molecular probe data to verify the presence of the appropriate microorganisms and laboratory microcosm tests may provide this type of information. A more detailed geochemical analysis may also be warranted to assess sustainability.

Transport models can be very helpful in analyzing and visualizing the data and expected plume conditions for comparison to field data. Transport models may be needed to predict the future state of the plume so that decisions can be made in a timely fashion and then confirmed through the long-term monitoring portion of MNA implementation. A simple transport model such as BIOCHLOR can be helpful as a screening tool in analyzing and visualizing the data and expected plume conditions for comparison to field data. However, BIOCHLOR may not be sufficient to describe the flow conditions in a heterogeneous aquifer. As such, more complex numerical modeling is more likely to be needed as part of MNA evaluation for Scenario 9 sites. To support this more complex analysis, more detailed field measurements may be required. The chart below summarizes an approach for analyzing data at sites depending on whether the concentration data indicates that the plume is decreasing, stable, or increasing and the source type. As noted in the table, as the source gets stronger and the plume is less likely to be decreasing in extent, more information is needed to support selection of an MNA remedy.

\begin{tabular}{|c|c|c|c|}
\hline & \multicolumn{3}{|c|}{ PLUME STATUS } \\
\hline $\begin{array}{l}\text { CONTAMINANT } \\
\text { CONCENTRATIONS/GEOCHEMICAL } \\
\text { STATUS }\end{array}$ & $\begin{array}{l}\text { DECREASING OR } \\
\text { PROBABLY } \\
\text { DECREASING }\end{array}$ & STABLE & $\begin{array}{l}\text { INCREASING, } \\
\text { PROBABLY } \\
\text { INCREASING, OR } \\
\text { PERTURBED }^{1}\end{array}$ \\
\hline Weak Source & - Mass loss & $\begin{array}{l}\text { - Mass loss } \\
\text { - Geochemical footprints } \\
\text { - Simple model }\end{array}$ & $\begin{array}{l}\text { - } \text { Mass loss } \\
\text { - } \quad \text { Coochemical footprints } \\
\text { Special Studies }\end{array}$ \\
\hline Strong Source & $\begin{array}{l}\text { - Mass loss } \\
\text { - Geochemical footprints }\end{array}$ & $\begin{array}{l}\text { - } \text { Mass loss } \\
\text { - } \text { Geochemical footprints } \\
\text { Special Studies }\end{array}$ & $\begin{array}{l}\text { - } \text { Mass loss } \\
\text { - } \quad \text { Geochemical footprints } \\
\text { Special Studies }\end{array}$ \\
\hline
\end{tabular}

${ }^{1}$ For instance if the plume has been impacted by a previous remedy such as P\&T.

To demonstrate mass loss construct these graphics:

- Concentration vs. time plots at individual wells;

- Concentration vs. distance plots, with multiple lines for different sampling events through time;

- Plume maps showing plume extent at different times (i.e., either panel maps, or one map with several plume boundaries for different times).

To show geochemical footprints make tables or figures that show:

- Daughter product production from abiotic reactions;

- Presence of primary substrate for co-metabolic reactions;

- Chloride product (this may not work at many sites, however, due to background chloride);

- Moderate to high dissolved oxygen concentrations (shows geochemical conditions area OK);

- No or limited methane production (shows geochemical conditions area OK).

To perform modeling, typical tools include the following:

- Simple transport model (analytical model, e.g., BIOCHLOR, BIOBALANCE ${ }^{1}$ );

- Comprehensive transport model (numerical model, e.g., RT3D). 


\begin{tabular}{|c|c|c|c|c|c|c|}
\hline $\begin{array}{c}\text { Simple } \\
\text { Fast }\end{array}$ & $\begin{array}{c}\text { Simple } \\
\text { Slow }\end{array}$ & $\begin{array}{c}\text { Heterogeneous } \\
\text { Slow }\end{array}$ & $\begin{array}{c}\text { Fractured/ } \\
\text { Porous Rock }\end{array}$ \\
\hline
\end{tabular}

If a special study is needed, some of the following may be applicable:

- Carbon/chlorine isotope analysis (indicator of degradation processes)

- Molecular probes (indicators of microbial activity)

- Microcosm tests (determine the reaction processes occurring at the site).

${ }^{1}$ Developed by Groundwater Services (www.gsi-net.com), with the support of the Savannah River National Laboratory and DOE, to evaluate monitored natural attenuation at chlorinated solvent sites.

\section{WHAT ABOUT COSTS AND ENHANCEMENTS?}

Costs for evaluating and implementing MNA for this scenario are primarily dependent on the following items.

- $\quad$ Source strength - Stronger and longer lasting sources will be more costly especially due to the high groundwater flow rate.

- $\quad$ Depth to the plume and size of the plume - Deeper, larger plumes require more and costlier monitoring wells.

- $\quad$ Travel time to the receptor - Plumes closer to receptors will require more frequent monitoring especially with the high groundwater flow rate.

- $\quad$ Plume stability - Less stable plumes require more rigorous evaluation and monitoring to select and implement MNA especially with the high groundwater flow rate.

- $\quad$ Nature of Heterogeneities - More heterogeneous aquifers may require a larger number of monitoring locations and more detailed analysis of flow and transport as part of evaluating MNA.

MNA may be a viable single remedy for the site. If it is determined that MNA may not meet remediation goals, a good first option is to evaluate the potential use of sustainable enhancements (enhanced attenuation). The objective being to adjust the attenuation conditions sufficiently such that the plume is controlled. If the enhancements are insufficient to control the plume, source control treatment may be required. In general, enhanced attenuation is less likely a viable option for a fast flow regime with a strong source, unless source treatment is undertaken initially. The sections below discuss options for enhanced attenuation and source control related to this scenario.

\section{Key Enhanced Attenuation Concepts}

Enhanced attenuation must be carefully designed in a heterogeneous flow system. Application of enhancements may be difficult due to the fast groundwater flow conditions. Enhancements are presented organized by the different zones in which they may be applied: source zone (reduction of contaminant mass flux to plume); plume (enhanced attenuation processes); or discharge zone (enhanced attenuation processes). Within the source zone, enhancements can be applied as a hydraulic manipulation or as a passive source reduction (active source control is discussed in the next section). Within the plume and discharge zone, either biological (microbial or plant based) or abiotic (abiotic degradation, reactive barriers, sorption) attenuation processes can be enhanced. A description of potential enhanced attenuation approaches, and their applicability to Scenario 9 sites, is shown below. More detailed information about each technology listed below is available in Early et al., (2005). 


$\mid$

\begin{tabular}{|c|c|}
\hline $\begin{array}{c}\text { Heterogeneous } \\
\text { Slow }\end{array}$ & $\begin{array}{c}\text { Fractured/ } \\
\text { Porous Rock }\end{array}$ \\
\hline
\end{tabular}

\begin{tabular}{|l|c|c|}
\hline \multicolumn{3}{|c}{ GEOCHEMICAL SETTING } \\
\hline Aerobic & Anoxic & Anaerobic \\
\hline
\end{tabular}

\section{Enhancement Summary}

\begin{tabular}{|c|c|c|}
\hline Enhancement & Description & Applicability to Scenario 9 Sites \\
\hline \multicolumn{3}{|c|}{ SOURCE ZONE ENHANCEMENTS } \\
\hline $\begin{array}{l}\text { Surface water or } \\
\text { groundwater } \\
\text { interception/diversion }\end{array}$ & $\begin{array}{l}\text { Use of interception } \\
\text { trenches or wells, } \\
\text { surface covers, or } \\
\text { phyto-covers (plants) } \\
\text { to reduce water flux } \\
\text { through source area }\end{array}$ & $\begin{array}{l}\text { May be more difficult in high flow rate conditions. May be difficult in } \\
\text { heterogeneous conditions. Likely more applicable using surface } \\
\text { covers or phyto-covers if the source is primarily within the vadose } \\
\text { zone. }\end{array}$ \\
\hline Physical containment & $\begin{array}{l}\text { Use of grout walls and } \\
\text { other physical } \\
\text { containment }\end{array}$ & $\begin{array}{l}\text { Potentially applicable depending on the geometry of the source } \\
\text { zone. }\end{array}$ \\
\hline Passive extraction & $\begin{array}{l}\text { Use of passive soil } \\
\text { vapor extraction }\end{array}$ & Useful if the source is primarily within the vadose zone. \\
\hline $\begin{array}{l}\text { Enhanced } \\
\text { biodegradation }\end{array}$ & $\begin{array}{l}\text { Injection of long-term } \\
\text { dissolved oxygen } \\
\text { source }\end{array}$ & $\begin{array}{l}\text { Well suited for aerobic sites if oxygen concentrations are marginal } \\
\text { in some areas; enhances existing aerobic biodegradation reactions. } \\
\text { Complex hydrogeology can make application difficult. Faster } \\
\text { groundwater flow may require larger amounts of oxygen than in } \\
\text { slower groundwater if electron donors are carried into the treatment } \\
\text { zone. }\end{array}$ \\
\hline $\begin{array}{l}\text { Enhanced } \\
\text { biodegradation }\end{array}$ & $\begin{array}{l}\text { Injection of electron } \\
\text { donor (e.g., HRC, } \\
\text { molasses, vegetable } \\
\text { oil) to enhance } \\
\text { microbial degradation } \\
\text { of the source. }\end{array}$ & $\begin{array}{l}\text { Typically more appropriate for anaerobic sites; stimulates anaerobic } \\
\text { contaminant biodegradation reactions. Potentially useful at aerobic } \\
\text { sites in source area to convert contaminants such as PCE and TCE } \\
\text { into contaminants such as DCE and VC that are degradable under } \\
\text { aerobic conditions. Need careful control of process to avoid } \\
\text { depleting all of the oxygen for the plume and eliminating the } \\
\text { potential for aerobic reactions. Complex hydrogeology can make } \\
\text { application difficult. Faster groundwater flow may require larger } \\
\text { amounts of electron donor than in slower groundwater if electron } \\
\text { acceptors are carried into the treatment zone. }\end{array}$ \\
\hline $\begin{array}{l}\text { Permeable reactive } \\
\text { barrier }\end{array}$ & $\begin{array}{l}\text { Use of zero valent } \\
\text { iron, reduced } \\
\text { sediment iron, } \\
\text { enhanced partitioning, } \\
\text { or biological barrier to } \\
\text { attenuate some of the } \\
\text { contaminant flux at } \\
\text { the downgradient } \\
\text { edge of the source } \\
\text { area. }\end{array}$ & $\begin{array}{l}\text { Barriers typically use anaerobic reactions. Influent of dissolved } \\
\text { oxygen is problematic for the barrier and the aquifer down gradient } \\
\text { of the barrier will be depleted in oxygen. Thus, barriers that use } \\
\text { anaerobic reactions are not typically suitable for aerobic sites. May } \\
\text { be more difficult and expensive to construct due to heterogeneous } \\
\text { conditions. May be more difficult and expensive to construct due to } \\
\text { fast flow regime. Faster groundwater flows potentially result in } \\
\text { more contaminant and competing electron acceptors passing } \\
\text { through the barrier, requiring greater thickness to achieve desired } \\
\text { treatment levels. }\end{array}$ \\
\hline
\end{tabular}




\begin{tabular}{|l|l|l|}
\hline Aerobic & Anoxic & Anaerobic \\
\hline
\end{tabular}

\begin{tabular}{|c|c|c|}
\hline Enhancement & Description & Applicability to Scenario 9 Sites \\
\hline \multicolumn{3}{|c|}{ PLUME AND DISCHARGE ZONE ENHANCEMENTS } \\
\hline $\begin{array}{l}\text { Enhanced } \\
\text { biodegradation }\end{array}$ & $\begin{array}{l}\text { Injection of long-term } \\
\text { dissolved oxygen } \\
\text { source }\end{array}$ & $\begin{array}{l}\text { Well suited for aerobic sites if oxygen concentrations are marginal } \\
\text { in some areas; enhances existing aerobic biodegradation reactions. } \\
\text { Complex hydrogeology can make application difficult. Faster } \\
\text { groundwater flow may require larger amounts of oxygen than in } \\
\text { slower groundwater if electron donors are carried into the treatment } \\
\text { zone. }\end{array}$ \\
\hline $\begin{array}{l}\text { Enhanced } \\
\text { biodegradation }\end{array}$ & $\begin{array}{l}\text { Injection of electron } \\
\text { donor (e.g., HRC, } \\
\text { molasses, vegetable } \\
\text { oil) to enhance } \\
\text { microbial degradation } \\
\text { of the source. }\end{array}$ & $\begin{array}{l}\text { Typically more appropriate for anaerobic sites; stimulates anaerobic } \\
\text { contaminant biodegradation reactions. Potentially useful at aerobic } \\
\text { sites in source area to convert contaminants such as PCE and TCE } \\
\text { into contaminants such as DCE and VC that are degradable under } \\
\text { aerobic conditions. Need careful control of process to avoid } \\
\text { depleting all of the oxygen for the plume and eliminating the } \\
\text { potential for aerobic reactions. Complex hydrogeology can make } \\
\text { application difficult. Faster groundwater flow may require larger } \\
\text { amounts of electron donor than in slower groundwater if electron } \\
\text { acceptors are carried into the treatment zone. }\end{array}$ \\
\hline $\begin{array}{l}\text { Permeable reactive } \\
\text { barrier }\end{array}$ & $\begin{array}{l}\text { Use of zero valent } \\
\text { iron, reduced } \\
\text { sediment iron, } \\
\text { enhanced partitioning, } \\
\text { or biological barrier to } \\
\text { attenuate some of the } \\
\text { contaminant flux } \\
\text { within the plume. }\end{array}$ & $\begin{array}{l}\text { Barriers typically use anaerobic reactions. Influent of dissolved } \\
\text { oxygen is problematic for the barrier and the aquifer down gradient } \\
\text { of the barrier will be depleted in oxygen. Thus, barriers that use } \\
\text { anaerobic reactions are not typically suitable for aerobic sites. May } \\
\text { be more difficult and expensive to construct due to heterogeneous } \\
\text { conditions. May be more difficult and expensive to construct due to } \\
\text { fast flow regime. Faster groundwater flows potentially result in } \\
\text { more contaminant and competing electron acceptors passing } \\
\text { through the barrier, requiring greater thickness to achieve desired } \\
\text { treatment levels. }\end{array}$ \\
\hline Phytoextraction & $\begin{array}{l}\text { Use of plants to } \\
\text { extract contaminants } \\
\text { from near surface } \\
\text { groundwater }\end{array}$ & $\begin{array}{l}\text { Heterogeneous aquifer conditions may make application difficult. } \\
\text { May be less effective for fast groundwater flow conditions. }\end{array}$ \\
\hline $\begin{array}{l}\text { Plant-based hydraulic } \\
\text { control (plume } \\
\text { enhancement only) }\end{array}$ & $\begin{array}{l}\text { Use of plants to } \\
\text { control hydraulic } \\
\text { gradient and slow } \\
\text { groundwater }\end{array}$ & $\begin{array}{l}\text { Heterogeneous aquifer conditions may make application difficult. } \\
\text { May be less effective for fast groundwater flow conditions. }\end{array}$ \\
\hline
\end{tabular}




\begin{tabular}{|c|c|c|c|c|}
\hline $\begin{array}{c}\text { Simple } \\
\text { Fast }\end{array}$ & $\begin{array}{c}\text { Simple } \\
\text { Slow }\end{array}$ & $\begin{array}{c}\text { Heterogeneous } \\
\text { Slow }\end{array}$ & $\begin{array}{c}\text { Fractured/ } \\
\text { Porous Rock }\end{array}$ \\
\hline
\end{tabular}

\begin{tabular}{|l|c|c|}
\hline \multicolumn{3}{|c}{ GEOCHEMICAL SETTING } \\
\hline Aerobic & Anoxic & Anaerobic \\
\hline
\end{tabular}

\section{Key Source Control Concepts}

A description of potential source control measures, and their applicability to Scenario 9 sites, is shown below. Note that source control measures are unlikely to achieve complete restoration at a site, and some source material is always left behind after treatment (U.S. EPA, 2003; McGuire et al., 2006).

\begin{tabular}{|c|c|c|c|}
\hline Example Technology & $\begin{array}{l}\text { Performance } \\
\left(25^{\mathrm{th}}-75^{\text {th }} \text { Percentile }\right. \\
\% \text { reduction in parent } \\
\text { compound })^{1}\end{array}$ & $\begin{array}{l}\frac{\text { Unit Cost }}{25^{\text {th }}-75^{\text {th }}} \\
\text { Percentile } \\
\text { (\$/yrd) })^{2}\end{array}$ & Applicability to Scenario 9 Sites \\
\hline In-situ biodegradation & $73-99$ & $27-152$ & $\begin{array}{l}\text { Potentially well suited for aerobic sites through addition of } \\
\text { co-substrate for aerobic degradation or potentially through } \\
\text { use of anaerobic reactions depending on how this action } \\
\text { impacts the downgradient geochemical conditions. Complex } \\
\text { hydrogeology can make application difficult. Faster } \\
\text { groundwater flow may require larger amounts of electron } \\
\text { donor than in slower groundwater if electron acceptors are } \\
\text { carried into the treatment zone. Least expensive treatment } \\
\text { option. }\end{array}$ \\
\hline Thermal treatment & $68-99.9$ & $48-129$ & $\begin{array}{l}\text { Does not appear to disrupt MNA after treatment. } \\
\text { Heterogeneous aquifer conditions may make application } \\
\text { difficult. }\end{array}$ \\
\hline Chemical oxidation & $70-97$ & $47-194$ & $\begin{array}{l}\text { Removes more total CVOCs than enhanced biodegradation } \\
\text { but shows more rebound }{ }^{1} \text {. Heterogeneous aquifer } \\
\text { conditions may make application difficult. May be suitable } \\
\text { for aerobic sites. }\end{array}$ \\
\hline Surfactant/cosolvents & $92-98$ & $118-1322$ & $\begin{array}{l}\text { High treatment efficiency but much higher cost. Costs reflect } \\
\text { some expensive pilot-scale projects. Heterogeneous aquifer } \\
\text { conditions may make application difficult. Some } \\
\text { surfactants/cosolvents can serve as electron donors for } \\
\text { subsequent anaerobic biodegradation reactions. }\end{array}$ \\
\hline Air sparging & - & - & $\begin{array}{l}\text { May be a viable alternative depending on the site geology } \\
\text { (e.g., contamination in an unconfined aquifer). } \\
\text { Heterogeneous aquifer conditions may make application } \\
\text { difficult. }\end{array}$ \\
\hline $\begin{array}{l}\text { Pump and Treat source } \\
\text { containment }\end{array}$ & NA & & $\begin{array}{l}\text { Due to the high groundwater flow rate, a large system may } \\
\text { be required. This approach does not reduce mass } \\
\text { significantly compared to the rate of mass loss without P\&T } \\
\text { and may need to be operated for a long time. }\end{array}$ \\
\hline
\end{tabular}




\begin{tabular}{|c|c|c|c|c|}
\hline $\begin{array}{c}\text { Simple } \\
\text { Fast }\end{array}$ & $\begin{array}{c}\text { Simple } \\
\text { Slow }\end{array}$ & $\begin{array}{c}\text { Heterogeneous } \\
\text { Fast }\end{array}$ & $\begin{array}{c}\text { Heterogeneous } \\
\text { Slow }\end{array}$ & $\begin{array}{c}\text { Fractured/ } \\
\text { Porous Rock }\end{array}$ \\
\hline
\end{tabular}

GEOCHEMICAL SETTING

\section{SCENARIO NUMBER 10}

Slower Flow With

Significant

Heterogeneities

and

Anaerobic

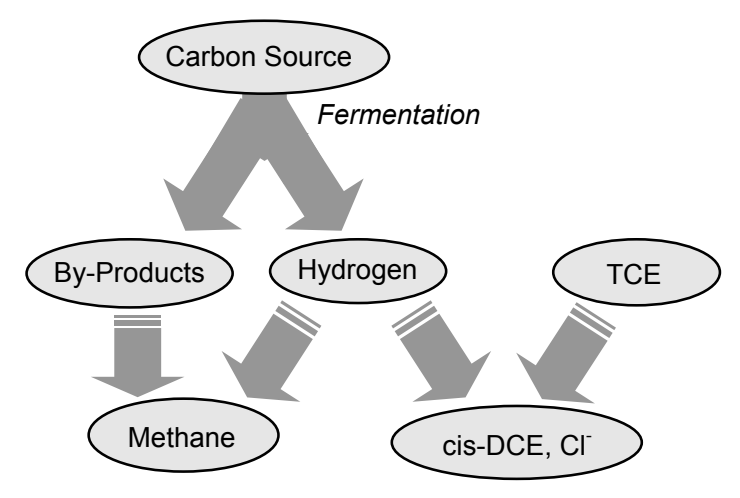

July 2006 


\begin{tabular}{|c|c|c|c|c|c|c|c|}
\hline $\begin{array}{l}\text { Simple } \\
\text { Fast }\end{array}$ & $\begin{array}{l}\text { Simple } \\
\text { Slow }\end{array}$ & $\begin{array}{c}\text { Heterogeneous } \\
\text { Fast }\end{array}$ & $\begin{array}{l}\text { Heterogeneous } \\
\text { Slow }\end{array}$ & $\begin{array}{l}\text { Fractured/ } \\
\text { Porous Rock }\end{array}$ & Aerobic & Anoxic & Anaerobic \\
\hline
\end{tabular}

\section{SCENARIO DESCRIPTION: SLOW FLOW WITH SIGNIFICANT HETEROGENEITIES AND ANAEROBIC}

The hydrogeologic setting and geochemical setting below define the basic scenario type. This scenario has the following characteristics:

\section{Hydrogeologic Setting}

"Slower Flow With Significant Heterogeneities" Hydrogeology:

- Potentially multiple hydrogeologic units

- Wide distribution of hydraulic conductivity

- Relatively low groundwater seepage velocity

(see Section 2.1 and Appendix 1 for more information)

\section{Geochemical Setting}

"Anaerobic" Geochemistry:

- Dissolved oxygen and redox are low

- Low to moderate concentrations of competing electron acceptors (nitrate, sulfate)

- Methane being produced.

(see Sections 2.2 for more information)

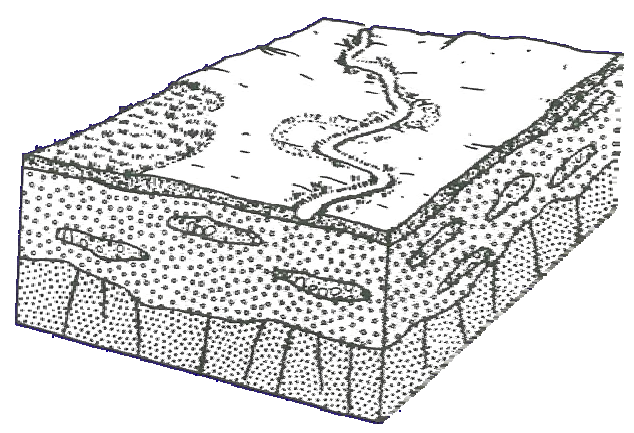

Block Diagram of Swamp/Marsh Aquifer from DRASTIC System

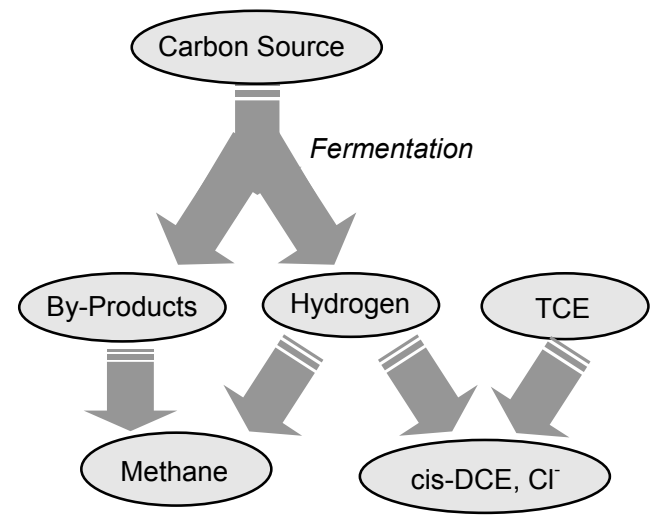

Example Reactions for "Anaerobic" Geochemical Setting 


\begin{tabular}{|c|c|c|c|c|c|c|c|}
\hline $\begin{array}{c}\text { Simple } \\
\text { Fast }\end{array}$ & $\begin{array}{c}\text { Simple } \\
\text { Slow }\end{array}$ & $\begin{array}{c}\text { Heterogeneous } \\
\text { Fast }\end{array}$ & $\begin{array}{c}\text { Heterogeneous } \\
\text { Slow }\end{array}$ & $\begin{array}{c}\text { Fractured/ } \\
\text { Porous Rock }\end{array}$
\end{tabular}$\quad$\begin{tabular}{ccc} 
Aerobic & Anoxic & Anaerobic \\
\hline
\end{tabular}

\section{KEY DECHLORINATION REACTIONS}

\section{Reaction Overview}

The chart to the right shows which reactions are likely to occur, which occur but at a slow rate, which may occur under specific conditions, and which are unlikely to occur.

\section{Typically Biodegradable Parent Compounds}

These compounds are typically degradable under anaerobic conditions:

- PCE

- TCE

- 1,1,1-TCA

- 1,2-DCA

- $\mathrm{CT}$

\section{Typical Daughter Products \\ Daughter products that may be present depending on the parent compound and the reactions listed to the right: \\ - TCE \\ - cis 1,2-DCE \\ - VC \\ - 1,2-DCA \\ - 1,1-DCE \\ - $\mathrm{CF}$}

See Section 5.1 for more information about reactions

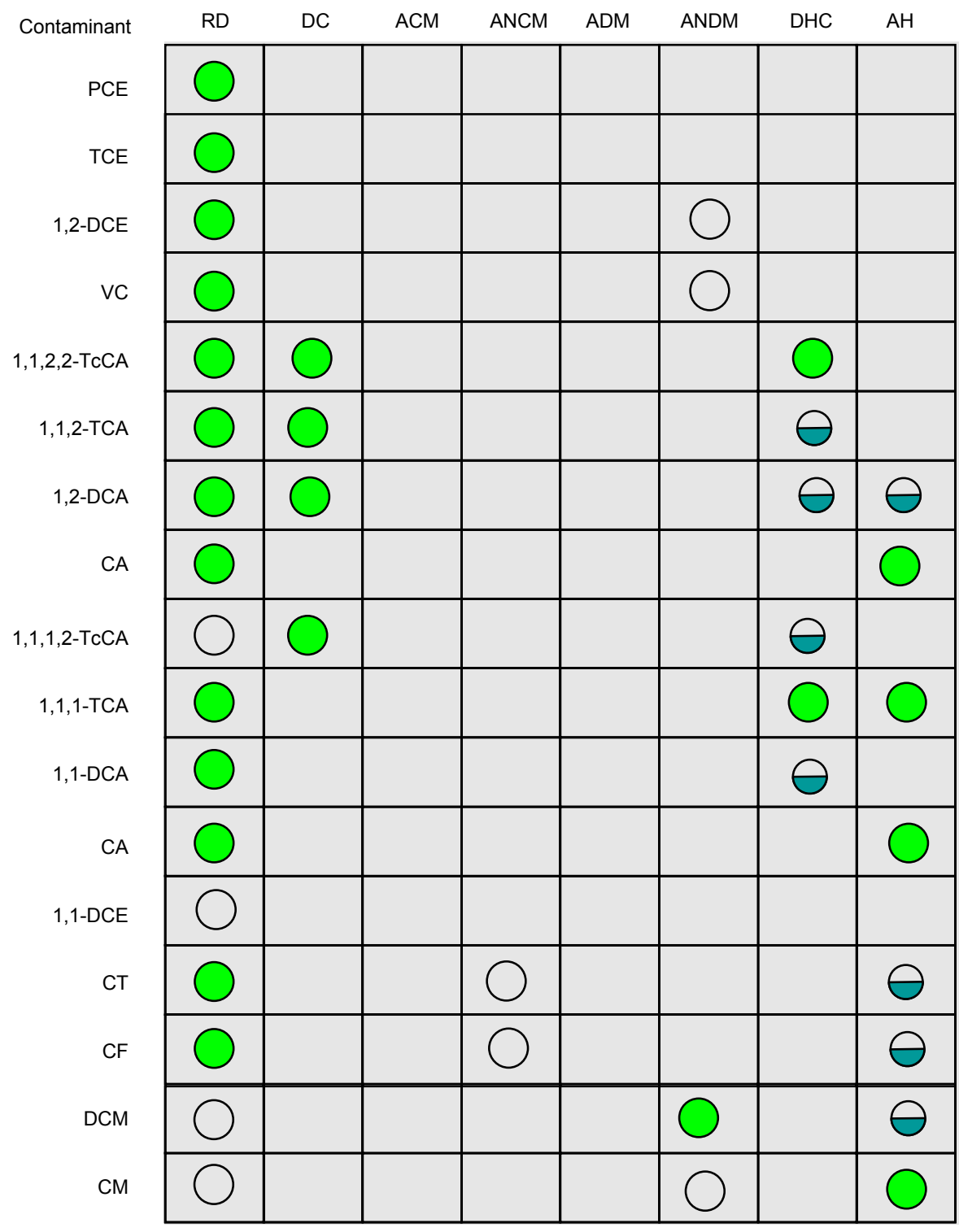

REACTIONS

\begin{tabular}{|ll|}
\hline Key: & \\
\hline & Highly Likely to occur \\
& Highly likely to occur, but a slow rate \\
& Hay occur under specific conditions \\
\hline
\end{tabular}

\begin{tabular}{|c|l|}
\hline ACM & Aerobic Co-Metabolism \\
\hline ANCM & Anaerobic Co-Metabolism \\
\hline ADM & Aerobic Direct Metabolism \\
\hline ANDM & Anaerobic Direct Metabolism \\
\hline DHC & Dehydrochlorination (abiotic) \\
\hline AH & Abiotic Hydrolysis \\
\hline DC & Dichloroelimination (biotic) \\
RD & Reductive Dechlorination (hydrogenolysis) \\
\hline
\end{tabular}




\begin{tabular}{|c|c|c|c|c|}
\hline $\begin{array}{c}\text { Simple } \\
\text { Fast }\end{array}$ & $\begin{array}{c}\text { Simple } \\
\text { Slow }\end{array}$ & $\begin{array}{c}\text { Heterogeneous } \\
\text { Fast }\end{array}$ & $\begin{array}{c}\text { Heterogeneous } \\
\text { Slow }\end{array}$ & $\begin{array}{c}\text { Fractured/ } \\
\text { Porous Rock }\end{array}$ \\
\hline
\end{tabular}

GEOCHEMICAL SETTING

\section{EFFECT OF MODIFYING FACTORS}

\begin{tabular}{|c|c|}
\hline MODIFYING FACTOR & KEY POINTS \\
\hline Source Str & $\begin{array}{l}\text { IF YOU HAVE A STRONG SOURCE: } \\
\text { - Plumes (both parent and daughter compounds) may be longer } \\
\text { - } \quad \text { Source zones may persist for longer periods of time } \\
\text { - More likely to need EA or source control measures } \\
\text { IF YOU HAVE A MODERATE SOURCE: } \\
\text { - Intermediate condition between Strong and Weak Source } \\
\text { IF YOU HAVE A WEAK SOURCE: } \\
\text { - Plumes may be shorter } \\
\text { - } \quad \text { Source zones may not persist as long } \\
\text { - MNA alone or MNA with EA more likely to be sufficient }\end{array}$ \\
\hline Source & $\begin{array}{l}\text { IF YOU HAVE MOSTLY A VADOSE ZONE SOURCE: } \\
\text { - Source may appear small due to dilution but can be large } \\
\text { - } \quad \text { Sources in clay vadose zones will be weaker but more long-lived than } \\
\text { sandy vadose zone } \\
\text { - Plumes will be thinner and closer to water table } \\
\text { IF YOU HAVE A SUBMERGED SOURCE: } \\
\text { - Complex hydrogeology means matrix diffusion may be important } \\
\text { - Source mass flux can decrease relatively rapidly as DNAPL fingers } \\
\text { - } \quad \text { dissolve } \\
\text { IF YOU HAVE A MIXED SOURCE: } \\
\text { - May have combination of the factors above }\end{array}$ \\
\hline Travel Time to $F$ & $\begin{array}{l}\text { CLOSE RECEPTORS (<2 YEARS TRAVEL TIME) } \\
\text { - More intensive monitoring system likely to be needed due to potential } \\
\text { - } \\
\text { Morious consequences in event of failure of MNA/EA. } \\
\text { MODERATE RECEPTORS ( }>2 \text { but < } 5 \text { YEARS TRAVEL TIME) } \\
\text { - Represents middle-ground case } \\
\text { DISTANT RECEPTORS ( }>5 \text { YEARS TRAVEL TIME) } \\
\text { - Less intensive monitoring system likely to be needed due to lower } \\
\text { - } \text { potential for serious consequences in event of failure of MNA/EA. }\end{array}$ \\
\hline Plume Stability & $\begin{array}{l}\text { EXPANDING OR PERTURBED }{ }^{1} \text { PLUME } \\
-\quad \text { More intensive monitoring system likely to be needed } \\
\text { STABLE PLUME } \\
\text { - Represents middle-ground case } \\
\text { SHRINKING PLUME } \\
\text { - Less intensive monitoring system likely to be needed } \\
\text { - MNA alone likely to be sufficient } \\
{ }^{1} \text { For instance if the plume has been impacted by a previous remedy such as P\&T. }\end{array}$ \\
\hline
\end{tabular}




\begin{tabular}{|c|c|c|c|c|c|c|c|}
\hline $\begin{array}{c}\text { Simple } \\
\text { Fast }\end{array}$ & $\begin{array}{c}\text { Simple } \\
\text { Slow }\end{array}$ & $\begin{array}{c}\text { Heterogeneous } \\
\text { Fast }\end{array}$ & $\begin{array}{c}\text { Heterogeneous } \\
\text { Slow }\end{array}$ & $\begin{array}{c}\text { Fractured/ } \\
\text { Porous Rock }\end{array}$ \\
\hline
\end{tabular}

\section{WILL MNA WORK?}

\section{Potential for MNA Processes to Control Plume}

This scenario often is well suited for natural attenuation processes to manage the contaminants in the plume or plume segment. The anaerobic conditions almost always mean that biodegradation processes are active.

In a slow-flowing heterogeneous aquifer where anaerobic conditions are present uniformly throughout the plume, relatively low rates of contaminant degradation can stabilize the plume. With the typical reductive dechlorination processes that are occurring under these conditions, it would be expected that the daughter product plume would be larger than the parent product plume because the degradation rate of daughters is typically slower than the rate for the parents.

The plume shape will be controlled by the heterogeneities in the plume segment. Plumes can be difficult to delineate, and can have complex, 3-dimensional shapes. Matrix diffusion effects in low-permeability zones can result in slowerthan-expected plume growth, which can be mistaken for mass destruction.

At some Scenario 10 sites, "DCE stall" may be of concern. DCE stall is an informal term typically used to describe conditions at chlorinated ethene sites where the cis-1,2-DCE "stalls outs" or exhibits a very low conversion rate to VC. This DCE "stall" condition has been ascribed to a variety of factors, including:

- $\quad$ Lack of the necessary microbiological communities that are required to degrade cis-1,2-DCE to VC;

- The direct conversion of cis-1,2-DCE to carbon dioxide, which makes it appear that cis-1,2-DCE is not being biodegraded because VC is not being produced; but in fact the cis-1,2-DCE is being biodegraded by direct oxidation to carbon dioxide;

- $\quad$ Conditions which are anaerobic enough to support the conversion of TCE to cis-1,2-DCE but not anaerobic enough to support the conversion from cis-1,2-DCE to VC by reductive dechlorination;

- Toxicity effects caused at sites where sulfate reducers are producing hydrogen sulfide $\left(\mathrm{H}_{2} \mathrm{~S}\right)$, but the $\mathrm{H}_{2} \mathrm{~S}$ is not being precipitated fast enough by ferrous iron (a by-product of ferric iron reduction) to prevent toxicity effects in the cis-1,2-DCE degraders.

While the cause of cis-1,2-DCE stall is still being evaluated by a number of researchers, the main implication is that at some chlorinated ethene sites, cis-1,2-DCE plumes are expanding and not being controlled. DCE "stall" does not affect long-term sustainability of a reaction, but does determine if natural attenuation processes are sufficient to prevent migration of the plume.

\section{Key Sustainability Concept}

Because lower rates of contaminant degradation are needed to stabilize a plume under slow-flowing aquifer conditions, and because the delivery of competing electron acceptors is reduced, sustainability of these reactions over the life of the source is a less critical issue compared to Scenario 1 (Simple Fast Flow and Anaerobic). However, sufficient natural substrate (e.g., organic matter) or co-contaminants that can act as a substrate are needed to sustain degradation. In a heterogeneous system, care should be taken to consider whether there are conduits for contaminant migration that do not have sufficient substrate available (e.g., a clean sand layer). The BIOBALANCE software system ${ }^{1}$ has a module designed to evaluate sustainability issues for anaerobic MNA reactions. Key input data are: i) mass fraction of solvents vs. donors in NAPL; OR ii) dissolved-phase concentrations of solvents and donors in the source zone.

1 Developed by Groundwater Services (www.gsi-net.com), with the support of the Savannah River National Laboratory and DOE, to evaluate monitored natural attenuation at chlorinated solvent sites. 


\begin{tabular}{|c|c|c|c|c|}
\hline $\begin{array}{c}\text { Simple } \\
\text { Fast }\end{array}$ & $\begin{array}{c}\text { Simple } \\
\text { Slow }\end{array}$ & $\begin{array}{c}\text { Heterogeneous } \\
\text { Fast }\end{array}$ & $\begin{array}{c}\text { Heterogeneous } \\
\text { Slow }\end{array}$ & $\begin{array}{c}\text { Fractured/ } \\
\text { Porous Rock }\end{array}$ \\
\hline
\end{tabular}

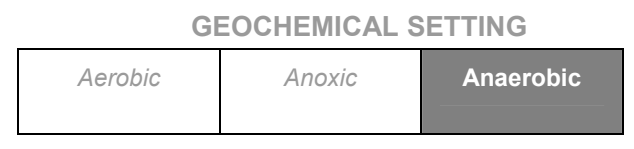

\section{HOW DO I CHARACTERIZE THIS TYPE OF SITE?}

\section{Actions Needed to Determine MNA Viability:}

The following are key considerations for evaluating the viability of MNA at a Slow Flow With Significant Heterogeneities and Anaerobic type site:

- both parent compound and daughter compounds need to be delineated (the anaerobic setting means that a number of daughter products will likely be generated);

- confirm that anaerobic conditions are present throughout the entire plume segment;

- determine the relative horizontal and vertical plume movement and whether there are layers (e.g., sandy units) where the plume movement is significantly greater than in other parts of the aquifer;

- determine if plume(s) are expanding/perturbed, stable, or shrinking;

- determine the location of any receptors (if present) and determine the travel time from the edge of the plume to these receptors;

- a mass-balance type evaluation of the source zone will help to determine if the electron donor supply is sustainable over the long term.

\section{Key Monitoring Concepts}

You will need to develop long, consistent concentration vs. time records to confirm if the plume is expanding, stable, or shrinking. However, the low groundwater velocity means that the risks are relatively low while monitoring is performed to determine if MNA is a viable plume management scenario or if modeling is used to select MNA and then confirmed with monitoring.

A more extensive monitoring system will likely be required to delineate the plume, because heterogeneities can result in wide distribution of hydraulic conductivity across the plume segment. Attention to vertical characteristics of the plume and monitoring within specific hydrologic layers may be particularly important. Plumes can have unusual shapes, such as apparent cross-gradient (regional gradient) flow patterns. This type of hydrogeologic setting benefits from plume delineation strategies using direct push approaches and adaptive plume delineation strategies.

\section{Key Uncertainty Concepts}

A key uncertainty for this scenario is associated with knowing that the plume is sufficiently delineated. In a heterogeneous subsurface, appropriate selection of monitoring locations is more difficult both horizontally and vertically within the plume. Because all of the MNA evaluation methods rely on a suitable conceptual model and data to describe the plume and subsurface properties, more data is typically required in a heterogeneous system to complete the evaluation at a level of detail acceptable to decision makers. 


\begin{tabular}{|c|c|c|c|c|}
\hline $\begin{array}{c}\text { Simple } \\
\text { Fast }\end{array}$ & $\begin{array}{c}\text { Simple } \\
\text { Slow }\end{array}$ & $\begin{array}{c}\text { Heterogeneous } \\
\text { Fast }\end{array}$ & $\begin{array}{c}\text { Heterogeneous } \\
\text { Slow }\end{array}$ & $\begin{array}{c}\text { Fractured/ } \\
\text { Porous Rock }\end{array}$ \\
\hline
\end{tabular}

\begin{tabular}{|l|c|c|}
\hline \multicolumn{2}{|c|}{ GEOCHEMICAL SETTING } \\
\hline Aerobic & Anoxic & Anaerobic \\
\hline
\end{tabular}

\section{HOW DO I ANALYZE DATA?}

In a slow-flowing aquifer, a plume is more likely to be shorter and will become stable (if it is going to) in a longer period of time than in faster-flow aquifers. Thus, plume maps, concentration vs. time at each well, and concentration vs. distance plots may be difficult to interpret to determine if the plume is expanding, stable, or shrinking. Longer and consistent temporal records of concentration at key wells (particularly at the leading edge of the plume) are important to evaluate MNA under the heterogeneous-slow flowing scenario. However, care must be taken to ensure that the monitoring network is sufficient for the heterogeneous conditions at the site. Concentrations should show an increase in the ratio of daughter to parent products with distance. If the plume edge is close to receptors, it may be necessary to provide additional data to verify anaerobic degradation processes are occurring and to assess the sustainability of these processes. Molecular probe data to verify the presence of the appropriate microorganisms and laboratory microcosm tests may provide this type of information. A more detailed geochemical analysis may also be warranted to assess sustainability.

Transport models can be very helpful in analyzing and visualizing the data and expected plume conditions for comparison to field data. Transport models may be needed to predict the future state of the plume so that decisions can be made in a timely fashion and then confirmed through the long-term monitoring portion of MNA implementation. A simple transport model such as BIOCHLOR can be helpful as a screening tool for analyzing and visualizing the data and expected plume conditions for comparison to field data. However, BIOCHLOR will not be sufficient to describe the flow conditions in a heterogeneous aquifer. As such, more complex numerical modeling is more likely to be needed as part of MNA evaluation for Scenario 10 sites. To support this more complex analysis, more detailed field measurements of hydraulic conditions may be required. The chart below summarizes an approach for analyzing data at sites depending on whether the concentration data indicates that the plume is decreasing, stable, or increasing and the source type. As noted in the table, as the source gets stronger and the plume is less likely to be decreasing in extent, more information is needed to support selection of an MNA remedy.

\begin{tabular}{|c|c|c|c|}
\hline & \multicolumn{3}{|c|}{ PLUME STATUS } \\
\hline $\begin{array}{l}\text { CONTAMINANT } \\
\text { CONCENTRATIONS/GEOCHEMICAL } \\
\text { STATUS }\end{array}$ & $\begin{array}{l}\text { DECREASING OR } \\
\text { PROBABLY } \\
\text { DECREASING }\end{array}$ & STABLE & $\begin{array}{l}\text { INCREASING, } \\
\text { PROBABLY } \\
\text { INCREASING, OR } \\
\text { PERTURBED } \\
\end{array}$ \\
\hline Weak Source & $\begin{array}{l}\text { - Mass loss } \\
\text { - Geochemical footprints }\end{array}$ & $\begin{array}{l}\text { - } \text { Mass loss } \\
\text { - } \text { Geochemical footprints } \\
\text { - Simple Model }\end{array}$ & $\begin{array}{l}\text { - Mass loss } \\
\text { - Geochemical footprints } \\
\text { - Comprehensive Model/ } \\
\text { Special Studies }\end{array}$ \\
\hline Strong Source & $\begin{array}{ll}\text { - } & \text { Mass loss } \\
\text { - } & \text { Geochemical footprints } \\
\text { - } & \text { Simple Model }\end{array}$ & $\begin{array}{l}\text { - } \text { Mass loss } \\
\text { - } \quad \text { Comprehensive Model/ } \\
\text { Special Studies }\end{array}$ & $\begin{array}{l}\text { - } \text { Mass loss } \\
\text { - } \text { Coochemical footprints } \\
\text { Special Studies }\end{array}$ \\
\hline
\end{tabular}

${ }^{1}$ For instance if the plume has been impacted by a previous remedy such as P\&T.

To demonstrate mass loss construct these graphics:

- Concentration vs. time plots at individual wells;

- Concentration vs. distance plots, with multiple lines for different sampling events through time;

- Plume maps showing plume extent at different times (i.e., either panel maps, or one map with several plume boundaries for different times).

To show geochemical footprints make tables or figures that show:

- Daughter product production;

- Ethene/Ethane production;

- Chloride production (this may not work at many sites, however, due to background chloride);

- Low dissolved oxygen (shows geochemical conditions are ok);

- Methane and iron(II) distribution (indicators of anaerobic activity);

- Nitrate and sulfate distribution (indicators of competing electron acceptors);

To perform modeling, typical tools include the following:

- Simple transport model (analytical model, e.g., BIOCHLOR, BIOBALANCE ${ }^{1}$ );

- Comprehensive transport model (numerical model, e.g., RT3D). 


\begin{tabular}{|c|c|c|c|c|c|}
\hline $\begin{array}{c}\text { Simple } \\
\text { Fast }\end{array}$ & $\begin{array}{c}\text { Simple } \\
\text { Slow }\end{array}$ & $\begin{array}{c}\text { Heterogeneous } \\
\text { Fast }\end{array}$ & $\begin{array}{c}\text { Heterogeneous } \\
\text { Slow }\end{array}$ & $\begin{array}{c}\text { Fractured/ } \\
\text { Porous Rock }\end{array}$ & Aerobic \\
\hline
\end{tabular}

If a special study is needed, some of the following may be applicable:

- Carbon/chlorine isotope analysis (indicator of degradation processes)

- Molecular probes (indicators of microbial activity)

- Microcosm tests (determine the reaction processes occurring at the site).

${ }^{1}$ Developed by Groundwater Services (www.gsi-net.com), with the support of the Savannah River National Laboratory and DOE, to evaluate monitored natural attenuation at chlorinated solvent sites.

\section{WHAT ABOUT COSTS AND ENHANCEMENTS?}

Costs for evaluating and implementing MNA for this scenario are primarily dependent on the following items.

- $\quad$ Source strength - Stronger and longer lasting sources will be more costly.

- $\quad$ Depth to the plume and size of the plume - Deeper, larger plumes require more and costlier monitoring wells.

- $\quad$ Nature of Heterogeneities - More heterogeneous aquifers may require a larger number of monitoring locations and more detailed analysis of flow and transport as part of evaluating MNA

- $\quad$ Travel time to the receptor - Plumes closer to receptors will require more frequent monitoring and over a long period of time with the slow groundwater flow rate.

- $\quad$ Plume stability - Less stable plumes require more rigorous evaluation and monitoring to select and implement MNA. With the slow groundwater flow rate, more rigorous evaluation or a longer period of monitoring may cause higher costs than for sites with a high groundwater flow rate.

MNA may be a viable single remedy for the site. If it is determined that MNA may not meet remediation goals, a good first option is to evaluate the potential use of sustainable enhancements (enhanced attenuation). The objective being to adjust the attenuation conditions sufficiently such that the plume is controlled. If the enhancements are insufficient to control the plume, source control treatment may be required. In general, enhanced attenuation is less likely a viable option for a fast flow regime with a strong source, unless source treatment is undertaken initially. The sections below discuss options for enhanced attenuation and source control related to this scenario.

\section{Key Enhanced Attenuation Concepts}

Enhanced attenuation must be carefully designed in a heterogeneous flow system. However, targeted application of enhancements may be highly effective due to the slow groundwater flow conditions. Enhancements are presented organized by the different zones in which they may be applied: source zone (reduction of contaminant mass flux to plume); plume (enhanced attenuation processes); or discharge zone (enhanced attenuation processes). Within the source zone, enhancements can be applied as a hydraulic manipulation or as a passive source reduction (active source control is discussed in the next section). Within the plume and discharge zone, either biological (microbial or plant based) or abiotic (abiotic degradation, reactive barriers, sorption) attenuation processes can be enhanced. A description of potential enhanced attenuation approaches, and their applicability to Scenario 10 sites, is shown below. More detailed information about each technology listed below is available in Early et al., (2005). 


\begin{tabular}{|c|c|c|c|c|}
\hline $\begin{array}{c}\text { Simple } \\
\text { Fast }\end{array}$ & $\begin{array}{c}\text { Simple } \\
\text { Slow }\end{array}$ & $\begin{array}{c}\text { Heterogeneous } \\
\text { Fast }\end{array}$ & $\begin{array}{c}\text { Heterogeneous } \\
\text { Slow }\end{array}$ & $\begin{array}{c}\text { Fractured/ } \\
\text { Porous Rock }\end{array}$ \\
\hline
\end{tabular}

\begin{tabular}{|l|c|c|}
\hline \multicolumn{2}{|c|}{ GEOCHEMICAL SETTING } \\
\hline Aerobic & Anoxic & Anaerobic \\
\hline
\end{tabular}

\section{Enhancement Summary}

\begin{tabular}{|c|c|}
\hline Enhancement & Description \\
\hline \multicolumn{2}{|c|}{ Source Zone Enhancements } \\
\hline $\begin{array}{l}\text { Surface water or } \\
\text { groundwater } \\
\text { interception/diversion }\end{array}$ & $\begin{array}{l}\text { Use of interception } \\
\text { trenches or wells, } \\
\text { surface covers, or } \\
\text { phyto-covers (plants) } \\
\text { to reduce water flux } \\
\text { through source area } \\
\text { and/or divert } \\
\text { unwanted electron } \\
\text { acceptors. }\end{array}$ \\
\hline Physical containment & $\begin{array}{l}\text { Use of grout walls and } \\
\text { other physical } \\
\text { containment }\end{array}$ \\
\hline Passive extraction & $\begin{array}{l}\text { Use of passive soil } \\
\text { vapor extraction }\end{array}$ \\
\hline $\begin{array}{l}\text { Enhanced } \\
\text { biodegradation }\end{array}$ & $\begin{array}{l}\text { Injection of electron } \\
\text { donor (e.g., HRC, } \\
\text { molasses, vegetable } \\
\text { oil) to enhance } \\
\text { microbial degradation } \\
\text { of the source. }\end{array}$ \\
\hline $\begin{array}{l}\text { Permeable reactive } \\
\text { barrier }\end{array}$ & $\begin{array}{l}\text { Use of zero valent } \\
\text { iron, reduced } \\
\text { sediment iron, } \\
\text { enhanced partitioning, } \\
\text { or biological barrier to } \\
\text { attenuate some of the } \\
\text { contaminant flux at } \\
\text { the downgradient } \\
\text { edge of the source } \\
\text { area. }\end{array}$ \\
\hline
\end{tabular}

Plume and Discharge Zone Enhancements

\begin{tabular}{|l|c|}
\hline $\begin{array}{l}\text { Enhanced } \\
\text { biodegradation }\end{array}$ & $\begin{array}{c}\text { Injection of electron } \\
\text { donor (e.g., HRC, } \\
\text { molasses, vegetable } \\
\text { oil) to enhance } \\
\text { microbial degradation } \\
\text { of the source. }\end{array}$ \\
\hline $\begin{array}{l}\text { Use of zero valent } \\
\text { iron, reduced } \\
\text { barrier }\end{array}$ \\
\hline $\begin{array}{l}\text { sediment iron, } \\
\text { enhanced partitioning, } \\
\text { or biological barrier to } \\
\text { attenuate some of the } \\
\text { contaminant flux } \\
\text { within the plume. }\end{array}$ \\
\hline Phytoextraction & $\begin{array}{c}\text { Use of plants to } \\
\text { extract contaminants } \\
\text { from near surface } \\
\text { groundwater }\end{array}$ \\
\hline $\begin{array}{l}\text { Plant-based hydraulic } \\
\text { control (plume } \\
\text { enhancement only) }\end{array}$ & $\begin{array}{c}\text { Use of plants to } \\
\text { control hydraulic } \\
\text { gradient and slow } \\
\text { groundwater }\end{array}$ \\
\hline
\end{tabular}

May be difficult in heterogeneous conditions. Likely more applicable using surface covers or phyto-covers if the source is primarily within the vadose zone.

Potentially applicable depending on the geometry of the source zone.

Useful if the source is primarily within the vadose zone.

Well suited for anaerobic sites; enhances existing biodegradation reactions. Suitable for most Scenario 10 sites. Complex hydrogeology can make application difficult. Slow groundwater flow may require relatively smaller amounts of electron donor.

May be more difficult and expensive to construct due to heterogeneous conditions. Slower groundwater flows potentially result in less contaminant and competing electron acceptors passing through the barrier, requiring less thickness to achieve desired treatment levels. Anaerobic conditions are helpful because oxygen concentration is low and will not disrupt barriers using anaerobic reactions.

Well suited for anaerobic sites; enhances existing biodegradation reactions. Suitable for most Scenario 10 sites. Complex hydrogeology can make application difficult. Slow groundwater flow may require relatively smaller amounts of electron donor.

May be more difficult and expensive to construct due to heterogeneous conditions. Slower groundwater flows potentially result in less contaminant and competing electron acceptors passing through the barrier, requiring less thickness to achieve desired treatment levels. Anaerobic conditions are helpful because oxygen concentration is low and will not disrupt barriers using anaerobic reactions.

Heterogeneous aquifer conditions may make application difficult.

Heterogeneous aquifer conditions may make application difficult. 


\begin{tabular}{|c|c|c|c|c|}
\hline $\begin{array}{c}\text { Simple } \\
\text { Fast }\end{array}$ & $\begin{array}{c}\text { Simple } \\
\text { Slow }\end{array}$ & $\begin{array}{c}\text { Heterogeneous } \\
\text { Fast }\end{array}$ & $\begin{array}{c}\text { Heterogeneous } \\
\text { Slow }\end{array}$ & $\begin{array}{c}\text { Fractured/ } \\
\text { Porous Rock }\end{array}$ \\
\hline
\end{tabular}

GEOCHEMICAL SETTING

\section{Key Source Control Concepts}

A description of potential source control measures, and their applicability to Scenario 10 sites, is shown below. Note that source control measures are unlikely to achieve complete restoration at a site, and some source material is always left behind after treatment (U.S. EPA, 2003; McGuire et al., 2006).

\begin{tabular}{|c|c|c|c|}
\hline$\frac{\text { Example }}{\text { Technology }}$ & $\begin{array}{l}\text { Performance } \\
\left(25^{\text {th }}-75^{\text {th }} \text { Percentile }\right. \\
\% \text { reduction in } \\
\text { parent compound })^{1}\end{array}$ & $\begin{array}{l}\text { Unit Cost } \\
25^{\text {th }}-75^{\text {th }} \\
\text { Percentile } \\
\text { (\$/yrd })^{2}\end{array}$ & Applicability to Scenario 10 Sites \\
\hline In-situ biodegradation & $73-99$ & $27-152$ & $\begin{array}{l}\text { Well suited for anaerobic sites; enhances existing } \\
\text { biodegradation reactions. Suitable for most Scenario } \\
10 \text { sites. Complex hydrogeology can make } \\
\text { application difficult. Slow groundwater flow may } \\
\text { require relatively smaller amounts of electron donor. }\end{array}$ \\
\hline Thermal treatment & $68-99.9$ & $48-129$ & $\begin{array}{l}\text { Does not appear to disrupt MNA after treatment. } \\
\text { Heterogeneous aquifer conditions may make } \\
\text { application difficult. }\end{array}$ \\
\hline Chemical oxidation & $70-97$ & $47-194$ & $\begin{array}{l}\text { Removes more total CVOCs than enhanced } \\
\text { biodegradation but shows more rebound }{ }^{1} \text {. Can } \\
\text { change geochemistry of Scenario } 10 \text { site to aerobic } \\
\text { conditions for some period after treatment. Can } \\
\text { change microbial population and composition. } \\
\text { Heterogeneous aquifer conditions may make } \\
\text { application difficult. May be more suitable for anoxic } \\
\text { or aerobic sites. }\end{array}$ \\
\hline Surfactant/cosolvents & $92-98$ & $118-1322$ & $\begin{array}{l}\text { High treatment efficiency but much higher cost. Costs } \\
\text { reflect some expensive pilot-scale projects. } \\
\text { Heterogeneous aquifer conditions may make } \\
\text { application difficult. Some surfactants/cosolvents can } \\
\text { serve as electron donors for subsequent anaerobic } \\
\text { biodegradation reactions. }\end{array}$ \\
\hline Air sparging & - & - & $\begin{array}{l}\text { Not recommended at most sites. Addition of oxygen } \\
\text { can disrupt anaerobic processes. Heterogeneous } \\
\text { aquifer conditions may make application difficult. }\end{array}$ \\
\hline $\begin{array}{l}\text { Pump and Treat } \\
\text { source containment }\end{array}$ & NA & & $\begin{array}{l}\text { This approach does not reduce mass significantly } \\
\text { compared to the rate of mass loss without P\&T and } \\
\text { may need to be operated for a long time. }\end{array}$ \\
\hline
\end{tabular}




\begin{tabular}{|c|c|c|c|c|c|c|c|}
\hline $\begin{array}{c}\text { Simple } \\
\text { Fast }\end{array}$ & $\begin{array}{c}\text { Simple } \\
\text { Slow }\end{array}$ & $\begin{array}{c}\text { Heterogeneous } \\
\text { Fast }\end{array}$ & $\begin{array}{c}\text { Heterogeneous } \\
\text { Slow }\end{array}$ & $\begin{array}{c}\text { Fractured/ } \\
\text { Porous Rock }\end{array}$ \\
\hline
\end{tabular}

\section{SCENARIO NUMBER 11}

\section{Slower Flow With Significant Heterogeneities and Anoxic}
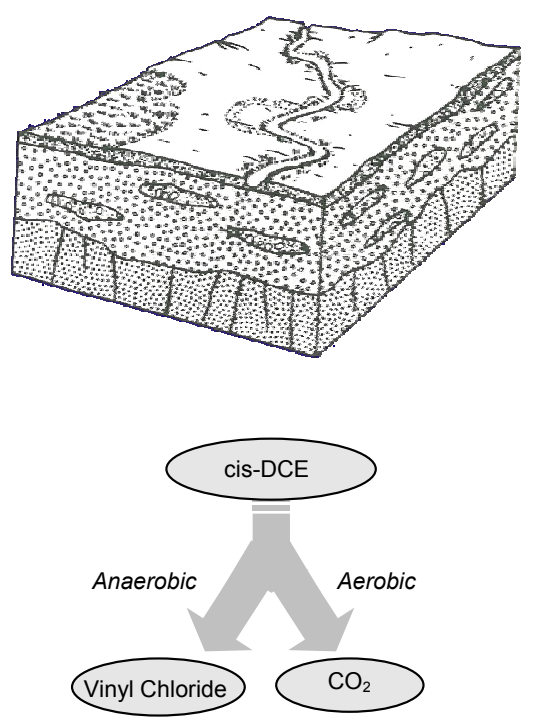


\begin{tabular}{|c|c|c|c|c|}
\hline $\begin{array}{c}\text { Simple } \\
\text { Fast }\end{array}$ & $\begin{array}{c}\text { Simple } \\
\text { Slow }\end{array}$ & $\begin{array}{c}\text { Heterogeneous } \\
\text { Fast }\end{array}$ & $\begin{array}{c}\text { Heterogeneous } \\
\text { Slow }\end{array}$ & $\begin{array}{c}\text { Fractured/ } \\
\text { Porous Rock }\end{array}$ \\
\hline
\end{tabular}

\begin{tabular}{|l|l|l|}
\multicolumn{2}{c}{ GEOCHEMICAL SETTING } \\
\begin{tabular}{|l|l|l|}
\hline Aerobic & Anoxic & Anaerobic \\
& & \\
\hline
\end{tabular}
\end{tabular}

\section{SCENARIO 11 DESCRIPTION: SLOW FLOW WITH SIGNIFICANT HETEROGENEITIES and ANOXIC}

The hydrogeologic setting and geochemical setting below define the basic scenario type. This scenario has the following characteristics:

\section{Hydrogeologic Setting}

"Slower Flow With Significant Heterogeneities" Hydrogeology:

- Potentially multiple hydrogeologic units

- Wide distribution of hydraulic conductivity

- Relatively low groundwater seepage velocity

(see Section 2.1 and Appendix 1 for more information)

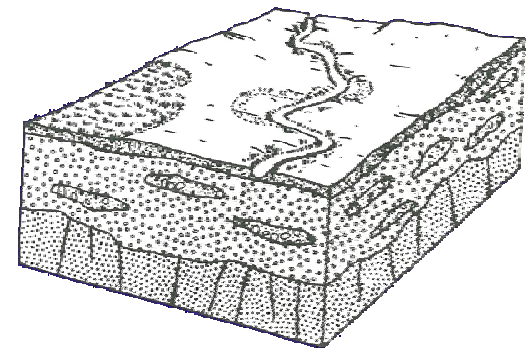

Block Diagram of Swamp/Marsh Aquifer from DRASTIC System

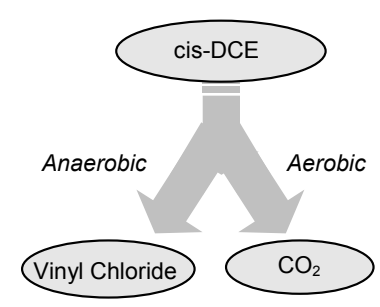

Example Reactions for "Anoxic" Geochemical Setting 
WSRC-STI-2006-00096, Rev. 1

October 2, 2006

\begin{tabular}{|c|c|c|c|c|c|c|c|}
\hline $\begin{array}{c}\text { Simple } \\
\text { Fast }\end{array}$ & $\begin{array}{c}\text { Simple } \\
\text { Slow }\end{array}$ & $\begin{array}{c}\text { Heterogeneous } \\
\text { Fast }\end{array}$ & $\begin{array}{c}\text { Heterogeneous } \\
\text { Slow }\end{array}$ & $\begin{array}{c}\text { Fractured/ } \\
\text { Porous Rock }\end{array}$ \\
\hline
\end{tabular}

\section{KEY DECHLORINATION REACTIONS}

\section{Reaction Overview}

The chart to the right shows which reactions are likely to occur, which occur but at a slow rate, which may occur under specific conditions, and which are unlikely to occur.

\section{Typically Biodegradable Parent Compounds \\ These compounds may be degradable under anoxic conditions: \\ - PCE \\ - TCE \\ - 1,1,1-TCA \\ - 1,2-DCA \\ - $\quad \mathrm{CT}$}

\section{Typical Daughter Products \\ Daughter products that may be present depending on the parent compound and the reactions listed to the right: \\ - TCE \\ - $\quad$ cis 1,2-DCE \\ - $\mathrm{VC}$ \\ - 1,2-DCA \\ - 1,1-DCE \\ - $\mathrm{CF}$}

See Section 5.1 for more information about reactions

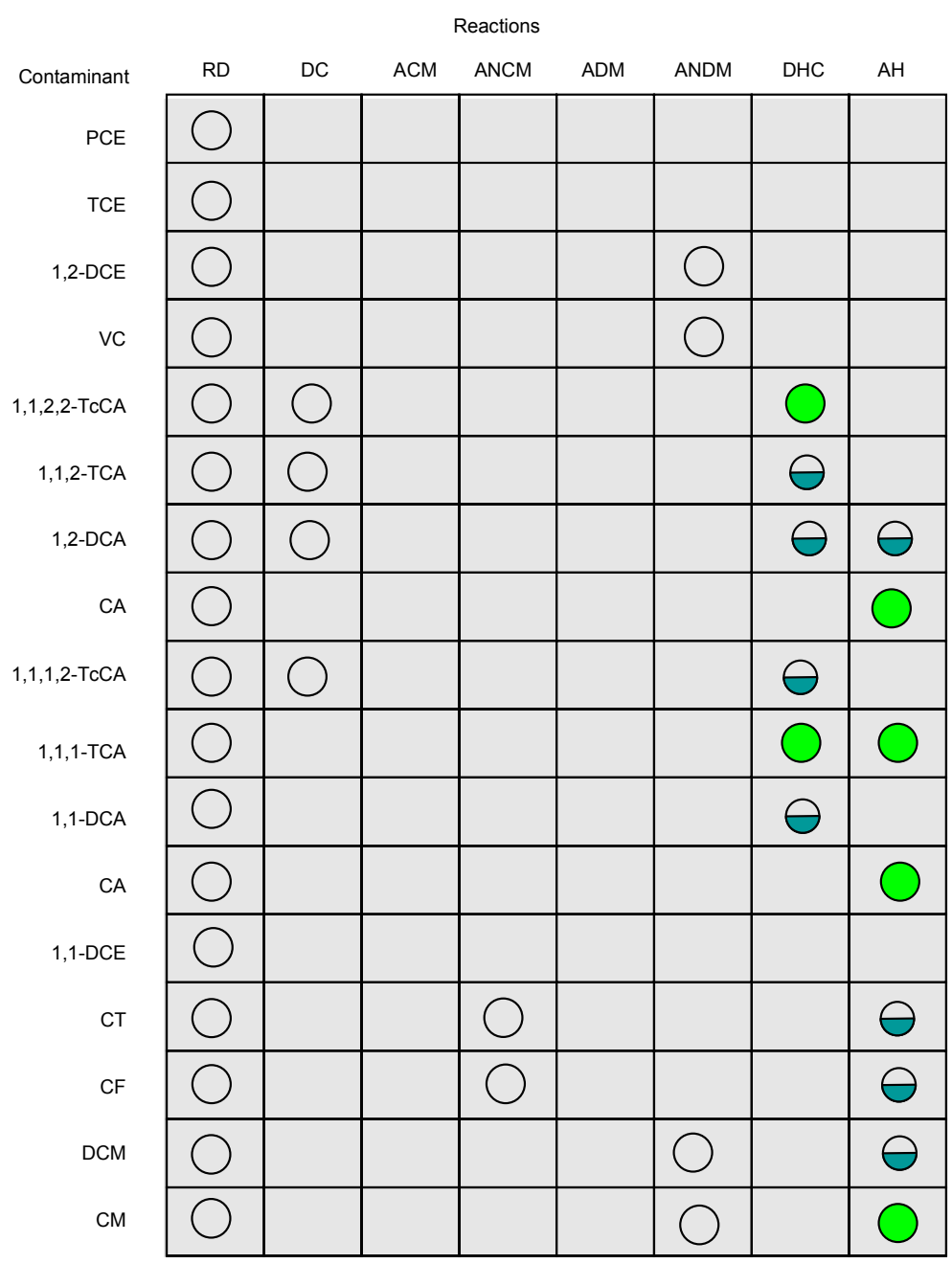

REACTIONS

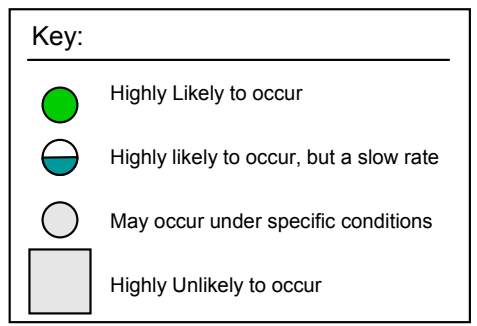

\begin{tabular}{|c|l|}
\hline ACM & Aerobic Co-Metabolism \\
\hline ANCM & Anaerobic Co-Metabolism \\
\hline ADM & Aerobic Direct Metabolism \\
\hline ANDM & Anaerobic Direct Metabolism \\
\hline DHC & Dehydrochlorination (abiotic) \\
\hline AH & Abiotic Hydrolysis \\
\hline DC & Dichloroelimination (biotic) \\
\hline RD & Reductive Dechlorination (hydrogenolysis) \\
\hline
\end{tabular}

Formatted: All caps

SCENARIO 11 - 3 
WSRC-STI-2006-00096, Rev. 1

October 2, 2006

GEOCHEMICAL SETTING

\begin{tabular}{|c|c|c|c|c|c|c|c|}
\hline $\begin{array}{c}\text { Simple } \\
\text { Fast }\end{array}$ & $\begin{array}{c}\text { Simple } \\
\text { Slow }\end{array}$ & $\begin{array}{c}\text { Heterogeneous } \\
\text { Fast }\end{array}$ & $\begin{array}{c}\text { Heterogeneous } \\
\text { Slow }\end{array}$ & $\begin{array}{c}\text { Fractured/ } \\
\text { Porous Rock }\end{array}$ \\
\hline
\end{tabular}

EFFECT OF MODIFYING FACTORS

\begin{tabular}{|c|c|}
\hline MODIFYING FACTOR & KEY POINTS \\
\hline Source Strength & $\begin{array}{l}\text { IF YOU HAVE A STRONG SOURCE: } \\
\text { - } \quad \text { Plumes (both parent and daughter compounds) may be longer } \\
\text { - } \quad \text { Source zones may persist for longer periods of time } \\
\text { IF YOU HAVE A MODERATE SOURCE: } \\
\text { - Intermediate condition between Strong and Weak Source } \\
\text { IF YOU HAVE A WEAK SOURCE: } \\
\text { - } \quad \text { Plumes may be shorter } \\
\text { - Source zones may not persist as long } \\
\text { - } \quad \text { MNA alone or MNA with EA more likely to be sufficient }\end{array}$ \\
\hline Source & $\begin{array}{l}\text { IF YOU HAVE MOSTLY A VADOSE ZONE SOURCE: } \\
\text { - Source may appear small due to dilution but can be large } \\
\text { - Sources in clay vadose zones will be weaker but more long-lived than } \\
\text { - } \\
\text { - Pandy vadose zone } \\
\text { IF YOU HAVE A SUBMERGED SOURCE: } \\
\text { - Complex hydrogeology means matrix diffusion may be important } \\
\text { - Source mass flux can decrease relatively rapidly as DNAPL fingers } \\
\text { - } \quad \text { dissolve } \\
\text { IF YOU HAVE A MIXED SOURCE: } \\
\text { - } \quad \text { May have combination of the factors above }\end{array}$ \\
\hline Travel Tim & $\begin{array}{l}\text { CLOSE RECEPTORS (<2 YEARS TRAVEL TIME) } \\
\text { - More intensive monitoring system likely to be needed due to potential } \\
\text { serious consequences in event of failure of MNA/EA. } \\
\text { More likely to need EA or source control measures } \\
\text { MODERATE RECEPTORS (>2 but < } 5 \text { YEARS TRAVEL TIME) } \\
\text { Represents middle-ground case } \\
\text { DISTANT RECEPTORS (>5 YEARS TRAVEL TIME) } \\
\text { - Less intensive monitoring system likely to be needed due to lower } \\
\text { - } \quad \text { MNAtential for serious consequences in event of failure of MNA/EA. }\end{array}$ \\
\hline Plume Stability & $\begin{array}{l}\text { EXPANDING OR PERTURBED }{ }^{1} \text { PLUME } \\
\text { - More intensive monitoring system likely to be needed } \\
\text { STABLE PLUME } \\
\text { - Represents middle-ground case } \\
\text { SHRINKING PLUME } \\
\text { - Less intensive monitoring system likely to be needed } \\
{ }^{1} \text { For instance if the plume has been impacted by a previous remedy such as P\&T. }\end{array}$ \\
\hline
\end{tabular}




\begin{tabular}{|c|c|c|c|c|c|c|c|}
\hline $\begin{array}{c}\text { Simple } \\
\text { Fast }\end{array}$ & $\begin{array}{c}\text { Simple } \\
\text { Slow }\end{array}$ & $\begin{array}{c}\text { Heterogeneous } \\
\text { Fast }\end{array}$ & $\begin{array}{c}\text { Heterogeneous } \\
\text { Slow }\end{array}$ & $\begin{array}{c}\text { Fractured/ } \\
\text { Porous Rock }\end{array}$ \\
\hline
\end{tabular}

\section{WILL MNA WORK?}

\section{Potential for MNA Processes to Control Plume}

Because at Scenario 11 sites there are not clear indicators that the type of conditions conducive to MNA are present, it is initially uncertain whether natural attenuation processes will be suitable to manage the contaminants in the plume or plume segment. Typically, more in-depth investigation of the site attenuation processes and more rigorous monitoring are needed to evaluate the extent of natural attenuation processes and the ability of MNA to meet the remediation objectives. Some form of enhanced attenuation may be needed to couple with MNA as the remedy.

In a slow-flowing heterogeneous aquifer, relatively low rates of contaminant degradation can stabilize the plume. If reductive dechlorination processes are occurring under these conditions, it would be expected that the daughter product plume would be larger than the parent product plume because the degradation rate of daughters is typically slower than the rate for the parents.

The plume shape will be controlled by the heterogeneities in the plume segment. Plumes can be difficult to delineate, and can have complex, 3-dimensional shapes. Matrix diffusion effects in low-permeability zones can result in slowerthan-expected plume growth, which can be mistaken for mass destruction.

If the plume is shown to be either stable or shrinking then natural attenuation processes (primarily reductive dechlorination) alone have been vigorous enough to date to prevent further migration of the plume or plume segment. Under these conditions MNA may be appropriate, but it may still be difficult to identify the specific attenuation mechanism under the anoxic geochemical conditions.

At some Scenario 11 sites, "DCE stall" may be of concern and an indication that conditions are not suitable for complete dechlorination of the source contaminants. DCE stall is an informal term typically used to describe conditions at chlorinated ethene sites where the cis-1,2-DCE "stalls outs" or exhibits a very low conversion rate to VC. This DCE "stall" condition has been ascribed to a variety of factors, including:

- $\quad$ Lack of the necessary microbiological communities that are required to degrade cis-1,2-DCE to VC;

- The direct conversion of cis-1,2-DCE to carbon dioxide, which makes it appear that cis-1,2-DCE is not being biodegraded because VC is not being produced; but in fact the cis-1,2-DCE is being biodegraded by direct oxidation to carbon dioxide;

- Conditions which are anaerobic enough to support the conversion of TCE to cis-1,2-DCE but not anaerobic enough to support the conversion from cis-1,2-DCE to VC by reductive dechlorination;

- $\quad$ Toxicity effects caused at sites where sulfate reducers are producing hydrogen sulfide $\left(\mathrm{H}_{2} \mathrm{~S}\right)$, but the $\mathrm{H}_{2} \mathrm{~S}$ is not being precipitated fast enough by ferrous iron (a by-product of ferric iron reduction) to prevent toxicity effects in the cis-1,2-DCE degraders.

While the cause of cis-1,2-DCE stall is still being evaluated by a number of researchers, the main implication is that at some chlorinated ethene sites, cis-1,2-DCE plumes are expanding and not being controlled. DCE "stall" does not affect long-term sustainability of a reaction, but does determine if natural attenuation processes are sufficient to prevent migration of the plume.

\section{Key Sustainability Concept}

If anaerobic reactions are occurring, sufficient natural substrate (e.g., organic matter) or co-contaminants that serve as electron donors and can act as a substrate are needed to sustain degradation for the reactions using the chlorinated solvent as an electron acceptor. In a heterogeneous system, care should be taken to consider whether there are conduits for contaminant migration that do not have sufficient substrate available (e.g., a clean sand layer). For some contaminants under anoxic conditions, biological reactions use the chlorinated solvent as the electron donor. At Scenario 11 sites, non-biologically catalyzed attenuation processes may be the primary attenuation processes. In this case, the processes are likely sustainable, but may be difficult to identify and quantify. 


\begin{tabular}{|c|c|c|c|c|c|c|c|}
\hline $\begin{array}{c}\text { Simple } \\
\text { Fast }\end{array}$ & $\begin{array}{c}\text { Simple } \\
\text { Slow }\end{array}$ & $\begin{array}{c}\text { Heterogeneous } \\
\text { Fast }\end{array}$ & $\begin{array}{c}\text { Heterogeneous } \\
\text { Slow }\end{array}$ & $\begin{array}{c}\text { Fractured/ } \\
\text { Porous Rock }\end{array}$ \\
\hline
\end{tabular}

\section{HOW DO I CHARACTERIZE THIS TYPE OF SITE?}

\section{Actions Needed to Determine MNA Viability}

The following are key considerations for evaluating the viability of MNA at a Slow Flow With Significant Heterogeneities and Anoxic type site:

- $\quad$ assess the site geochemical, hydraulic, and contaminant conditions in detail to assess the type and extent/rate of attenuation processes - this assessment may require significant effort depending on the site conditions

- $\quad$ determine the relative horizontal and vertical plume movement and whether there are layers (e.g., sandy units) where the plume movement is significantly greater than in other parts of the aquifer;

- $\quad$ both parent compound and daughter compounds need to be delineated (the anoxic setting means that a number of daughter products may be generated);

- $\quad$ determine if plume(s) are expanding/perturbed, stable, or shrinking;

- determine the location of any receptors (if present) and determine the travel time from the edge of the plume to these receptors;

- a mass-balance type evaluation of the source zone will help to determine if the electron donor supply is sustainable over the long term if the attenuation reactions are determined to be primarily anaerobic dechlorination with the contaminant acting as the electron acceptor.

\section{Key Monitoring Concepts}

You will need to develop long, consistent concentration vs. time records to confirm if the plume is expanding, stable, or shrinking. However, the low groundwater velocity means that the risks are relatively low while monitoring is performed to determine if MNA is a viable plume management scenario or if modeling is used to select MNA and then confirmed with monitoring.

A more extensive monitoring system will likely be required to delineate the plume, because heterogeneities can result in wide distribution of hydraulic conductivity across the plume segment. Attention to vertical characteristics of the plume and monitoring within specific hydrologic layers may be particularly important. Plumes can have unusual shapes, such as apparent cross-gradient (regional gradient) flow patterns. This type of hydrogeologic setting benefits from plume delineation strategies using direct push approaches and adaptive plume delineation strategies.

\section{Key Uncertainty Concepts}

A key uncertainty for this scenario is associated with knowing that the plume is sufficiently delineated. In a heterogeneous subsurface, appropriate selection of monitoring locations is more difficult both horizontally and vertically within the plume. Because all of the MNA evaluation methods rely on a suitable conceptual model and data to describe the plume and subsurface properties, more data is typically required in a heterogeneous system to complete the evaluation at a level of detail acceptable to decision makers. The anoxic geochemical setting may cause considerable uncertainty in evaluating MNA because it may be more difficult to identify and quantify the attenuation processes. 


\begin{tabular}{|c|c|c|c|c|c|c|}
\hline $\begin{array}{c}\text { Simple } \\
\text { Fast }\end{array}$ & $\begin{array}{c}\text { Simple } \\
\text { Slow }\end{array}$ & $\begin{array}{c}\text { Heterogeneous } \\
\text { Fast }\end{array}$ & $\begin{array}{c}\text { Heterogeneous } \\
\text { Slow }\end{array}$ & $\begin{array}{c}\text { Fractured/ } \\
\text { Porous Rock }\end{array}$ \\
\hline
\end{tabular}

\section{HOW DO I ANALYZE DATA?}

In a slow-flowing aquifer, a plume is more likely to be shorter and will become stable (if it is going to) in a longer period of time than in faster-flow aquifers. Thus, plume maps, concentration vs. time at each well, and concentration vs. distance plots may be difficult to interpret to determine if the plume is expanding, stable, or shrinking. Longer and consistent temporal records of concentration at key wells (particularly at the leading edge of the plume) are important to evaluate MNA under the heterogeneous-slow flowing scenario. However, care must be taken to ensure that the monitoring network is sufficient for the heterogeneous conditions at the site. Concentrations may not show a progression of parent to daughter products with distance. Thus, it is likely that contaminant monitoring over a period of time will be needed to establish trends in the plume size and concentration data. In some cases, this type of data will be sufficient for a fast-flowing aquifer with anoxic conditions.

Transport models can be very helpful in analyzing and visualizing the data and expected plume conditions for comparison to field data. Transport models may be needed to predict the future state of the plume so that decisions can be made in a timely fashion and then confirmed through the long-term monitoring portion of MNA implementation. A simple transport model such as BIOCHLOR can be helpful as a screening tool in analyzing and visualizing the data and expected plume conditions for comparison to field data. However, BIOCHLOR will not be sufficient to describe the range of attenuation processes that may be important under anoxic geochemical conditions and may not be sufficient to describe the flow conditions in a heterogeneous aquifer. As such, more complex numerical modeling is more likely to be needed as part of MNA evaluation for Scenario 8 sites. To support this more complex analysis, microcosm tests, molecular probes, and more detailed field measurements may be required. The chart below summarizes an approach for analyzing data at sites depending on whether the concentration data indicates that the plume is decreasing, stable, or increasing and the source type. As noted in the table, as the source gets stronger and the plume is less likely to be decreasing in extent, more information is needed to support an MNA remedy.

\begin{tabular}{|c|c|c|c|}
\hline & \multicolumn{3}{|c|}{ PLUME STATUS } \\
\hline $\begin{array}{l}\text { CONTAMINANT } \\
\text { CONCENTRATIONS/GEOCHEMICAL } \\
\text { STATUS }\end{array}$ & $\begin{array}{l}\text { DECREASING OR } \\
\text { PROBABLY } \\
\text { DECREASING }\end{array}$ & STABLE & $\begin{array}{l}\text { INCREASING, } \\
\text { PROBABLY } \\
\text { INCREASING, OR } \\
\text { PERTURBED }\end{array}$ \\
\hline Weak Source & - Mass loss & $\begin{array}{l}\text { - } \text { Mass loss } \\
\text { - Seochemical footprints } \\
\text { Studies }\end{array}$ & $\begin{array}{l}\text { - } \text { Mass loss } \\
\text { - } \text { Coochemical footprints } \\
\text { Special Studies }\end{array}$ \\
\hline Strong Source & $\begin{array}{ll}\text { - } & \text { Mass loss } \\
\text { - } & \text { Geochemical footprints } \\
\text { - } & \text { Simple Model }\end{array}$ & $\begin{array}{ll}\text { - } & \text { Mass loss } \\
\text { - } & \text { Geochemical footprints } \\
& \text { Special Studies }\end{array}$ & $\begin{array}{l}\text { - } \text { Mass loss } \\
\text { - } \quad \text { Coochemical footprints } \\
\quad \text { Special Studies }\end{array}$ \\
\hline
\end{tabular}

${ }^{1}$ For instance if the plume has been impacted by a previous remedy such as P\&T.

To demonstrate mass loss construct these graphics:

- Concentration vs. time plots at individual wells;

- Concentration vs. distance plots, with multiple lines for different sampling events through time;

- Plume maps showing plume extent at different times (i.e., either panel maps, or one map with several plume boundaries for different times).

To show geochemical footprints make tables or figures that show:

- Daughter product production

- Ethene/Ethane production;

- Chloride production (this may not work at many sites, however, due to background chloride)

- Low dissolved oxygen (shows anoxic geochemical conditions);

- Methane and iron(II) distribution (indicators of anaerobic activity);

- Nitrate and sulfate distribution (indicators of competing electron acceptors);

To perform modeling, typical tools include the following:

- Simple transport model (analytical model, e.g., BIOCHLOR, BIOBALANCE ${ }^{1}$ );

- Comprehensive transport model (numerical model, e.g., RT3D). 


\begin{tabular}{|c|c|c|c|c|c|c|}
\hline $\begin{array}{c}\text { Simple } \\
\text { Fast }\end{array}$ & $\begin{array}{c}\text { Simple } \\
\text { Slow }\end{array}$ & $\begin{array}{c}\text { Heterogeneous } \\
\text { Fast }\end{array}$ & $\begin{array}{c}\text { Heterogeneous } \\
\text { Slow }\end{array}$ & $\begin{array}{c}\text { Fractured/ } \\
\text { Porous Rock }\end{array}$ \\
\hline
\end{tabular}

If a special study is needed, some of the following may be applicable:

- Carbon/chlorine isotope analysis (indicator of degradation processes)

- Molecular probes (indicators of microbial activity when other geochemical indicators are ambiguous);

- Microcosm tests (determine the reaction processes occurring at the site).

1 Developed by Groundwater Services (www.gsi-net.com), with the support of the Savannah River National Laboratory and DOE, to evaluate monitored natural attenuation at chlorinated solvent sites.

\section{WHAT ABOUT COSTS AND ENHANCEMENTS?}

Costs for evaluating and implementing MNA for this scenario are primarily dependent on the following items.

- Source strength - Stronger and longer lasting sources will be more costly.

- Depth to the plume and size of the plume - Deeper, larger plumes require more and costlier monitoring wells.

- Travel time to the receptor - Plumes closer to receptors will require more frequent monitoring and over a long period of time with the slow groundwater flow rate.

- Plume stability - Less stable plumes require more rigorous evaluation and monitoring to select and implement MNA. With the slow groundwater flow rate, more rigorous evaluation or a longer period of monitoring may cause higher costs than for sites with a high groundwater flow rate.

- Extent of variability in geochemical conditions - More variability will likely require more characterization and monitoring to assess attenuation conditions within each different geochemical zone.

- Nature of Heterogeneities - More heterogeneous aquifers may require a larger number of monitoring locations and more detailed analysis of flow and transport as part of evaluating MNA.

MNA may be a viable single remedy for the site. If it is determined that MNA may not meet remediation goals, a good first option is to evaluate the potential use of sustainable enhancements (enhanced attenuation). The objective being to adjust the attenuation conditions sufficiently such that the plume is controlled. If the enhancements are insufficient to control the plume, source control treatment may be required. In general, enhanced attenuation is less likely a viable option for a fast flow regime with a strong source, unless source treatment is undertaken initially. The sections below discuss options for enhanced attenuation and source control related to this scenario.

\section{Key Enhanced Attenuation Concepts}

Enhanced attenuation must be carefully designed in a heterogeneous flow system. However, targeted application of enhancements may be highly effective due to the slow groundwater flow conditions. Enhancements are presented organized by the different zones in which they may be applied: source zone (reduction of contaminant mass flux to plume); plume (enhanced attenuation processes); or discharge zone (enhanced attenuation processes). Within the source zone enhancements can be applied as a hydraulic manipulation or as a passive source reduction (active source control is discussed in the next section). Within the plume and discharge zone, either biological (microbial or plant based) or abiotic (abiotic degradation, reactive barriers, sorption) attenuation processes can be enhanced. A description of potential enhanced attenuation approaches, and their applicability to Scenario 11 sites, is shown below. More detailed information about each technology listed below is available in Early et al., (2005). 
WSRC-STI-2006-00096, Rev. 1

October 2, 2006

HYDROGEOLOGIC SETTING

\begin{tabular}{|c|c|c|c|c|c|c|c|c|}
\hline $\begin{array}{c}\text { Simple } \\
\text { Fast }\end{array}$ & $\begin{array}{c}\text { Simple } \\
\text { Slow }\end{array}$ & $\begin{array}{c}\text { Heterogeneous } \\
\text { Fast }\end{array}$ & $\begin{array}{c}\text { Heterogeneous } \\
\text { Slow }\end{array}$ & $\begin{array}{c}\text { Fractured/ } \\
\text { Porous Rock }\end{array}$ \\
\hline
\end{tabular}

\section{Enhancement Summary}

Enhancement

\section{SOURCE ZONE ENHANCEMENTS}

Surface water or

groundwater

interception/diversion

trenches or wells, surface

covers, or phyto-covers

(plants) to reduce water

flux through source area

and/or divert unwanted

electron acceptors.

Physical containment

Use of grout walls and other physical containment

\section{Passive extraction}

Enhanced

biodegradation

(1)

\begin{tabular}{|c|c|}
\hline $\begin{array}{l}\text { Permeable reactive } \\
\text { barrier }\end{array}$ & $\begin{array}{l}\text { Use of zero valent iron, } \\
\text { reduced sediment iron, } \\
\text { enhanced partitioning, or } \\
\text { biological barrier to } \\
\text { attenuate some of the } \\
\text { contaminant flux at the } \\
\text { downgradient edge of the } \\
\text { source area. }\end{array}$ \\
\hline
\end{tabular}

PLUME AND DISCHARGE ZONE ENHANCEMENTS

Enhanced
biodegradation

Permeable reactive barrier

Injection of electron donor (e.g., HRC, molasses vegetable oil) to enhance microbial degradation of the source.

Use of zero valent iron, reduced sediment iron, enhanced partitioning, or biological barrier to attenuate some of the contaminant flux within the plume.

Phytoextraction

Plant-based hydraulic control (plume enhancement only)
Use of plants to extract contaminants from near surface groundwater

Use of plants to control hydraulic gradient and slow groundwater
May be difficult in heterogeneous conditions. Likely more applicable using surface covers or phyto-covers if the source is primarily within the vadose zone.

Potentially applicable depending on the geometry of the source zone.

Useful if the source is primarily within the vadose zone.

Potentially well suited for anoxic sites because oxygen concentrations are already low and anaerobic processes may be readily stimulated. Complex hydrogeology can make application difficult. Slow groundwater flow may require relatively smaller amounts of electron donor.

May be more difficult and expensive to construct due to heterogeneous conditions. Slower groundwater flows potentially result in less contaminant and competing electron acceptors passing through the barrier, requiring less thickness to achieve desired treatment levels. Anoxic conditions are helpful because oxygen concentration is low and will not disrupt barriers using anaerobic reactions.

Potentially well suited for anoxic sites because oxygen concentrations are already low and anaerobic processes may be readily stimulated. Complex hydrogeology can make application difficult. Slow groundwater flow may require relatively smaller amounts of electron donor.

May be more difficult and expensive to construct due to heterogeneous conditions. Slower groundwater flows potentially result in less contaminant and competing electron acceptors passing through the barrier, requiring less thickness to achieve desired treatment levels. Anoxic conditions are helpful because oxygen concentration is low and will not disrupt barriers using anaerobic reactions.

Heterogeneous aquifer conditions may make application difficult.

Heterogeneous aquifer conditions may make application difficult. 
WSRC-STI-2006-00096, Rev. 1

October 2, 2006

HYDROGEOLOGIC SETTING

\begin{tabular}{|c|c|c|c|c|}
\hline $\begin{array}{c}\text { Simple } \\
\text { Fast }\end{array}$ & $\begin{array}{c}\text { Simple } \\
\text { Slow }\end{array}$ & $\begin{array}{c}\text { Heterogeneous } \\
\text { Fast }\end{array}$ & $\begin{array}{c}\text { Heterogeneous } \\
\text { Slow }\end{array}$ & $\begin{array}{c}\text { Fractured/ } \\
\text { Porous Rock }\end{array}$ \\
\hline
\end{tabular}

GEOCHEMICAL SETTING

\begin{tabular}{|l|c|c|}
\hline \multicolumn{2}{|c|}{ GEOCHEMICAL SETTING } \\
\hline Aerobic & Anoxic & Anaerobic \\
\hline
\end{tabular}

\section{Key Source Control Concepts}

A description of potential source control measures, and their applicability to Scenario 11 sites, is shown below. Note that source control measures are unlikely to achieve complete restoration at a site, and some source material is always left behind after treatment (U.S. EPA, 2003; McGuire et al., 2006). <table format>

\begin{tabular}{|c|c|c|c|}
\hline Example Technology & $\begin{array}{l}\text { Performance } \\
\left(25^{\text {th }}-75^{\text {th }} \text { Percentile }\right. \\
\% \text { reduction in } \\
\text { parent compound })^{1}\end{array}$ & $\begin{array}{l}\frac{\text { Unit Cost }}{25^{\text {th }}-75^{\text {th }}} \\
\text { Percentile } \\
\text { (\$/yrd })^{2}\end{array}$ & Applicability to Scenario 11 Sites \\
\hline In-situ biodegradation & $73-99$ & $27-152$ & $\begin{array}{l}\text { Potentially well suited for anoxic sites because oxygen } \\
\text { concentrations are already low and anaerobic processes } \\
\text { may be readily stimulated. Complex hydrogeology can } \\
\text { make application difficult. Slow groundwater flow may } \\
\text { require relatively smaller amounts of electron donor. } \\
\text { Least expensive treatment option. }\end{array}$ \\
\hline Thermal treatment & $68-99.9$ & $48-129$ & $\begin{array}{l}\text { Does not appear to disrupt MNA after treatment. } \\
\text { Heterogeneous aquifer conditions may make application } \\
\text { difficult. }\end{array}$ \\
\hline Chemical oxidation & $70-97$ & $47-194$ & $\begin{array}{l}\text { Removes more total CVOCs than enhanced } \\
\text { biodegradation but shows more rebound }{ }^{1} \text {. Can change } \\
\text { geochemistry of Scenario } 11 \text { site to aerobic conditions for } \\
\text { some period after treatment. Can change microbial } \\
\text { population and composition. Heterogeneous aquifer } \\
\text { conditions may make application difficult. }\end{array}$ \\
\hline Surfactant/cosolvents & $92-98$ & $118-1322$ & $\begin{array}{l}\text { High treatment efficiency but much higher cost. Costs } \\
\text { reflect some expensive pilot-scale projects. } \\
\text { Heterogeneous aquifer conditions may make application } \\
\text { difficult. Some surfactants/cosolvents can serve as } \\
\text { electron donors for subsequent anaerobic biodegradation } \\
\text { reactions. }\end{array}$ \\
\hline Air sparging & - & - & $\begin{array}{l}\text { May be suitable if only limited biological attenuation is } \\
\text { occurring at a site. Addition of oxygen can disrupt } \\
\text { anaerobic processes that may be occurring. } \\
\text { Heterogeneous aquifer conditions may make application } \\
\text { difficult. }\end{array}$ \\
\hline $\begin{array}{l}\text { Pump and Treat source } \\
\text { containment }\end{array}$ & NA & & $\begin{array}{l}\text { This approach does not reduce mass significantly } \\
\text { compared to the rate of mass loss without P\&T and may } \\
\text { need to be operated for a long time. }\end{array}$ \\
\hline
\end{tabular}




\begin{tabular}{|c|c|c|c|c|c|c|c|}
\hline $\begin{array}{c}\text { Simple } \\
\text { Fast }\end{array}$ & $\begin{array}{c}\text { Simple } \\
\text { Slow }\end{array}$ & $\begin{array}{c}\text { Heterogeneous } \\
\text { Fast }\end{array}$ & $\begin{array}{c}\text { Heterogeneous } \\
\text { Slow }\end{array}$ & $\begin{array}{c}\text { Fractured/ } \\
\text { Porous Rock }\end{array}$ \\
\hline
\end{tabular}

\section{SCENARIO NUMBER 12}

Slower Flow With

Significant

Heterogeneities

and

Aerobic
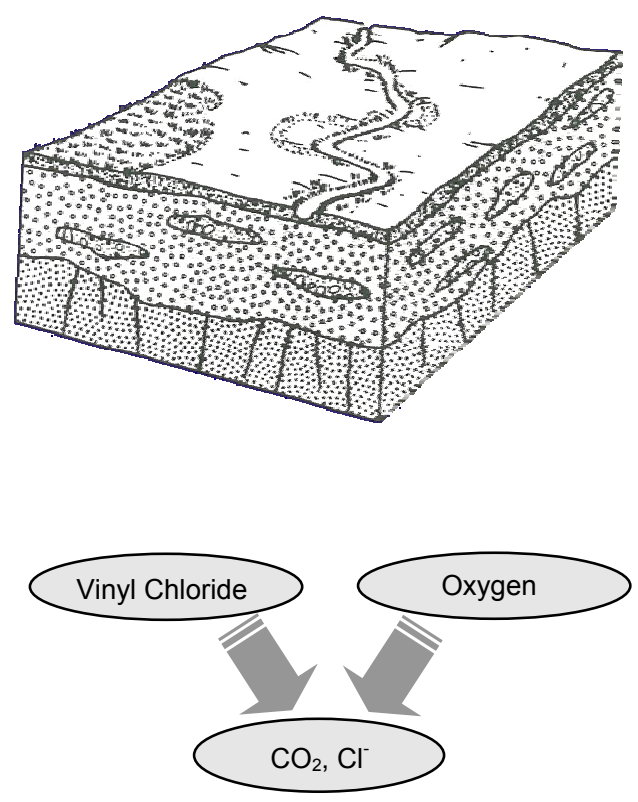


\begin{tabular}{|c|c|c|c|c|}
\hline $\begin{array}{c}\text { Simple } \\
\text { Fast }\end{array}$ & $\begin{array}{c}\text { Simple } \\
\text { Slow }\end{array}$ & $\begin{array}{c}\text { Heterogeneous } \\
\text { Fast }\end{array}$ & $\begin{array}{c}\text { Heterogeneous } \\
\text { Slow }\end{array}$ & $\begin{array}{c}\text { Fractured/ } \\
\text { Porous Rock }\end{array}$ \\
\hline
\end{tabular}

\section{SCENARIO 12 DESCRIPTION: SLOWER FLOW WITH SIGNIFICANT HETEROGENEITIES and AEROBIC}

The hydrogeologic setting and geochemical setting below define the basic scenario type. This scenario has the following characteristics:

\section{Hydrogeologic Setting}

"Slower Flow With Significant Heterogeneities" Hydrogeology:

- Potentially multiple hydrogeologic units

- Wide distribution of hydraulic conductivity

- Relatively low groundwater seepage velocity

(see Section 2.1 and Appendix 1 for more information)

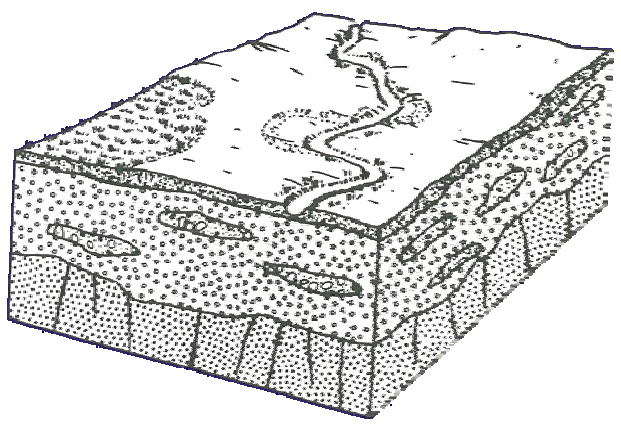

Block Diagram of Swamp/Marsh Aquifer from DRASTIC System

\section{Geochemical Setting}

"Aerobic" Geochemistry:

- Dissolved oxygen and redox are moderate to high

- Possible to have wide range of concentrations of competing electron acceptors (nitrate, sulfate)

- $\quad$ No or very limited presence of anaerobic indicators (e.g., methane)

(see Section 2.2 for more information)

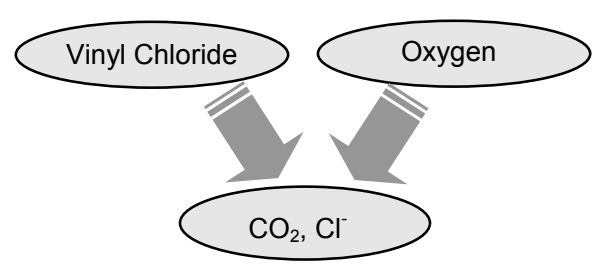

Example Reactions for "Aerobic" Geochemical Setting 


\begin{tabular}{|c|c|c|c|c|c|c|c|}
\hline $\begin{array}{c}\text { Simple } \\
\text { Fast }\end{array}$ & $\begin{array}{c}\text { Simple } \\
\text { Slow }\end{array}$ & $\begin{array}{c}\text { Heterogeneous } \\
\text { Fast }\end{array}$ & $\begin{array}{c}\text { Heterogeneous } \\
\text { Slow }\end{array}$ & $\begin{array}{c}\text { Fractured/ } \\
\text { Porous Rock }\end{array}$ \\
\hline
\end{tabular}

\section{KEY DECHLORINATION REACTIONS}

\section{Reaction Overview}

The chart to the right shows which reactions are likely to occur, which occur but at a slow rate, and which may occur under specific conditions, and which are unlikely to occur.

\section{Compounds Easier for Biological Degradation}

- $\quad$ cis 1,2-DCE

- $\mathrm{VC}$

- 1,2-DCA

- 1,1-DCE

- $\mathrm{DCM}$

- $\mathrm{CM}$

\section{Compounds More Difficult for Biological Degradation}

- PCE

- TCE

- $\quad$ CT

- $\mathrm{CF}$

See Section 5.1 for more information about reactions

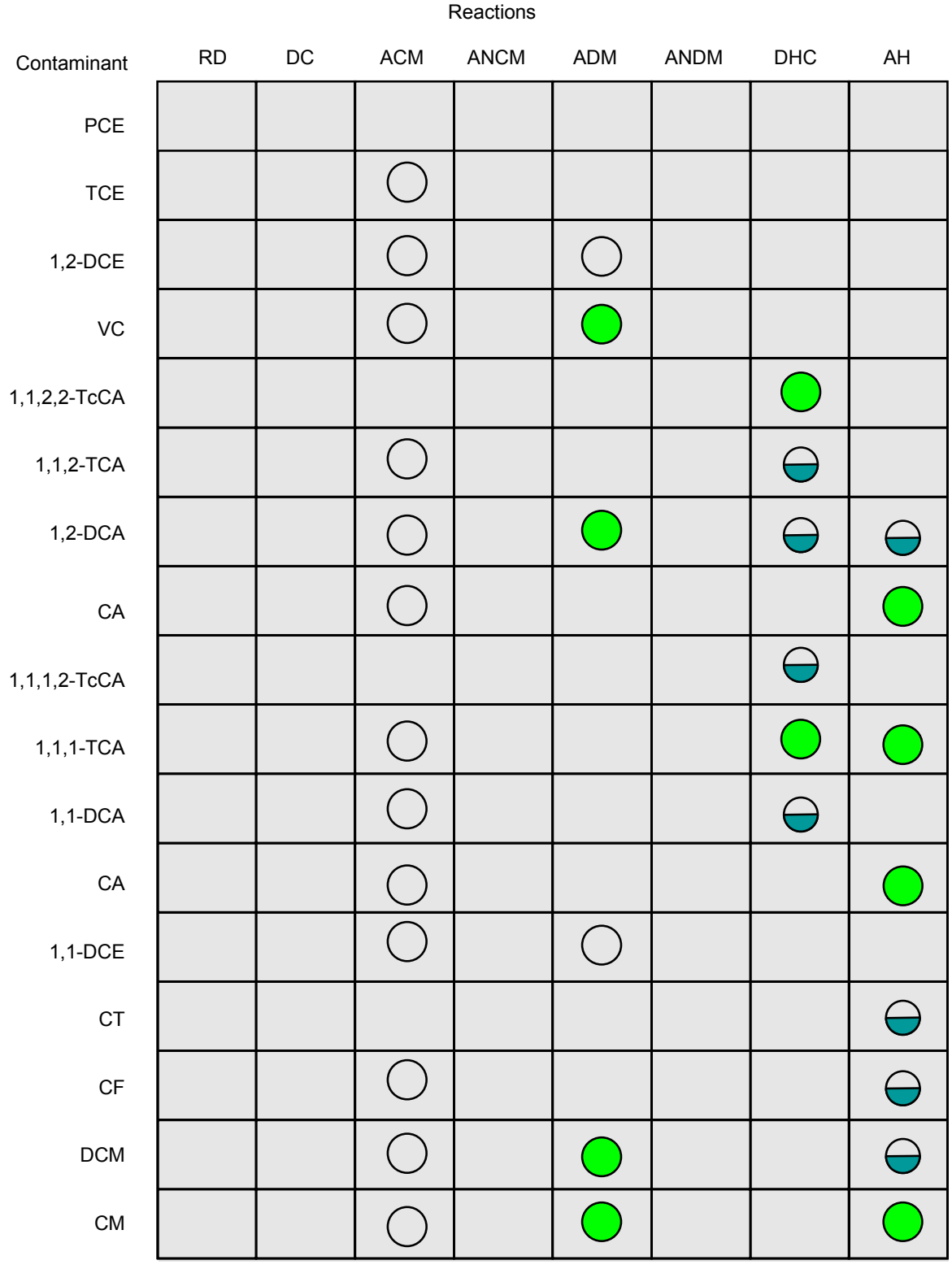

REACTIONS

Key:

\begin{tabular}{|c|l|}
\hline ACM & Aerobic Co-Metabolism \\
\hline ANCM & Anaerobic Co-Metabolism \\
\hline ADM & Aerobic Direct Metabolism \\
\hline ANDM & Anaerobic Direct Metabolism \\
\hline DHC & Dehydrochlorination (abiotic) \\
\hline AH & Abiotic Hydrolysis \\
\hline DC & Dichloroelimination (biotic) \\
\hline RD & Reductive Dechlorination (hydrogenolysis) \\
\hline
\end{tabular}




\begin{tabular}{|c|c|c|c|c|}
\hline $\begin{array}{c}\text { Simple } \\
\text { Fast }\end{array}$ & $\begin{array}{c}\text { Simple } \\
\text { Slow }\end{array}$ & $\begin{array}{c}\text { Heterogeneous } \\
\text { Fast }\end{array}$ & $\begin{array}{c}\text { Heterogeneous } \\
\text { Slow }\end{array}$ & $\begin{array}{c}\text { Fractured/ } \\
\text { Porous Rock }\end{array}$ \\
\hline
\end{tabular}

\section{EFFECT OF MODIFYING FACTORS}

\begin{tabular}{|c|c|}
\hline MODIFYING FACTOR & KEY POINTS \\
\hline Source Strength & $\begin{array}{l}\text { IF YOU HAVE A STRONG SOURCE: } \\
\text { - } \quad \text { Plumes (both parent and daughter compounds) may be longer } \\
\text { - } \quad \text { Source zones may persist for longer periods of time } \\
\text { - More likely to need EA or source control measures } \\
\text { IF YOU HAVE A MODERATE SOURCE: } \\
\text { - Intermediate condition between Strong and Weak Source } \\
\text { IF YOU HAVE A WEAK SOURCE: } \\
\text { - Plumes may be shorter } \\
\text { - } \quad \text { Source zones may not persist as long } \\
\text { - } \quad \text { MNA alone or MNA with EA more likely to be sufficient }\end{array}$ \\
\hline Source & $\begin{array}{l}\text { IF YOU HAVE MOSTLY A VADOSE ZONE SOURCE: } \\
\text { - Source may appear small due to dilution but can be large } \\
\text { - Sources in clay vadose zones will be weaker but more long-lived than } \\
\text { sandy vadose zone } \\
\text { - Plumes will be thinner and closer to water table } \\
\text { IF YOU HAVE A SUBMERGED SOURCE: } \\
\text { - Complex hydrogeology means matrix diffusion may be important } \\
\text { - Source mass flux can decrease relatively rapidly as DNAPL fingers } \\
\text { - } \quad \text { dissolve } \\
\text { IF YOU HAVE A MIXED SOURCE: } \\
\text { - May have combination of the factors above }\end{array}$ \\
\hline Travel Tim & $\begin{array}{l}\text { CLOSE RECEPTORS (<2 YEARS TRAVEL TIME) } \\
\text { - More intensive monitoring system likely to be needed due to potential } \\
\text { - } \\
\text { Merious consequences in event of failure of MNA/EA. } \\
\text { MODERATE RECEPTORS ( }>2 \text { but }<5 \text { YEARS TRAVEL TIME) } \\
\text { - Represents middle-ground case } \\
\text { DISTANT RECEPTORS ( }>5 \text { YEARS TRAVEL TIME) } \\
\text { - Less intensive monitoring system likely to be needed due to lower } \\
\text { - } \quad \text { MNtential for serious consequences in event of failure of MNA/EA. }\end{array}$ \\
\hline Plume Stability & $\begin{array}{l}\text { EXPANDING OR PERTURBED }{ }^{1} \text { PLUME } \\
-\quad \text { More intensive monitoring system likely to be needed } \\
\text { STABLE PLUME } \\
\text { - Represents middle-ground case } \\
\text { SHRINKING PLUME } \\
\text { - Less intensive monitoring system likely to be needed } \\
\text { - MNA alone likely to be sufficient } \\
{ }^{1} \text { For instance if the plume has been impacted by a previous remedy such as P\&T. }\end{array}$ \\
\hline
\end{tabular}




\begin{tabular}{|c|c|c|c|c|c|}
\hline $\begin{array}{c}\text { Simple } \\
\text { Fast }\end{array}$ & $\begin{array}{c}\text { Simple } \\
\text { Slow }\end{array}$ & $\begin{array}{c}\text { Heterogeneous } \\
\text { Fast }\end{array}$ & $\begin{array}{c}\text { Heterogeneous } \\
\text { Slow }\end{array}$ & $\begin{array}{c}\text { Fractured/ } \\
\text { Porous Rock }\end{array}$ & Aerobic \\
\hline
\end{tabular}

\section{WILL MNA WORK?}

\section{Potential for MNA Processes to Control Plume}

In slow-flowing heterogeneous plumes or plume segments where aerobic conditions are present uniformly throughout the plume, there is less likelihood that natural attenuation processes will result in short, stable or shrinking plumes than in anaerobic plumes or plume segments if parent compounds such as PCE and TCE are present. While TCE and some other parent compounds can be degraded biologically under aerobic conditions, these reactions are cometabolic reactions that require the presence of methane or another similar substrate that are typically not present in aerobic aquifers under natural conditions. Abiotic degradation processes will occur for some compounds, but may produce daughter products that cannot be readily degraded under aerobic conditions. Some compounds can be degraded directly by aerobic bacteria (e.g., DCE and VC). In summary, aerobic conditions are generally less conducive for managing chlorinated solvent plumes, except for a plume segment downgradient of an anaerobic plume segment where the contamination is dominated by reductive dechlorination daughter products such as cis-1,2DCE or VC that can be directly degraded under aerobic conditions.

The plume shape will be controlled by the heterogeneities in the plume segment. Plumes can be difficult to delineate, and can have complex, 3-dimensional shapes. Matrix diffusion effects in low-permeability zones can result in slower-than-expected plume growth, which can be mistaken for mass destruction.

\section{Key Sustainability Concept}

Direct aerobic biologic reactions and abiotic reactions are likely to be sustainable indefinitely.

Other biodegradation reactions that can occur under aerobic conditions are co-metabolic reactions that require oxygen and a primary substrate (such as methane). The probability that the supply of dissolved oxygen to the plume from upgradient sources (and plume re-aeration to a lesser degree) will be interrupted is relatively low. In a heterogeneous system, care should be taken to consider whether there are conduits for contaminant migration that do not have sufficient oxygen available. Additionally, changes in source structure over time could result in reduced delivery of the primary substrate, increasing the uncertainty in the long-term sustainability of a naturally occurring cometabolic reaction. 


\begin{tabular}{|c|c|c|c|c|c|c|c|}
\hline $\begin{array}{c}\text { Simple } \\
\text { Fast }\end{array}$ & $\begin{array}{c}\text { Simple } \\
\text { Slow }\end{array}$ & $\begin{array}{c}\text { Heterogeneous } \\
\text { Fast }\end{array}$ & $\begin{array}{c}\text { Heterogeneous } \\
\text { Slow }\end{array}$ & $\begin{array}{c}\text { Fractured/ } \\
\text { Porous Rock }\end{array}$ \\
\hline
\end{tabular}

\section{HOW DO I CHARACTERIZE THIS TYPE OF SITE?}

\section{Actions Needed to Determine MNA Viability:}

The following are key considerations for evaluating the viability of MNA at a Slow Flow With Significant Heterogeneities and Aerobic type site:

- trends for contaminant concentrations need to be established to assess whether attenuation is occurring (the aerobic setting means that a daughter products will likely not be available to assess whether attenuation processes are occurring);

- determine the relative horizontal and vertical plume movement and whether there are layers (e.g., sandy units) where the plume movement is significantly greater than in other parts of the aquifer;

- confirm that aerobic conditions are present throughout the entire plume/plume segment;

- $\quad$ determine if plume(s) are expanding/perturbed, stable, or shrinking;

- determine the location of any receptors (if present) and determine the travel time from the edge of the plume to these receptors.

\section{Key Monitoring Concepts}

You will need to develop long, consistent concentration vs. time records to confirm if the plume is expanding, stable, or shrinking. However, the low groundwater velocity means that the risks are relatively low while monitoring is performed to determine if MNA is a viable plume management scenario or if modeling is used to select MNA and then confirmed with monitoring.

A more extensive monitoring system will likely be required to delineate the plume, because heterogeneities can result in wide distribution of hydraulic conductivity across the plume segment. Attention to vertical characteristics of the plume and monitoring within specific hydrologic layers may be particularly important. Plumes can have unusual shapes, such as apparent cross-gradient (regional gradient) flow patterns. This type of hydrogeologic setting benefits from plume delineation strategies using direct push approaches and adaptive plume delineation strategies.

\section{Key Uncertainty Concepts}

A key uncertainty for this scenario is associated with knowing that the plume is sufficiently delineated. In a heterogeneous subsurface, appropriate selection of monitoring locations is more difficult both horizontally and vertically within the plume. Because all of the MNA evaluation methods rely on a suitable conceptual model and data to describe the plume and subsurface properties, more data is typically required in a heterogeneous system to complete the evaluation at a level of detail acceptable to decision makers.

Additionally, because daughter compounds for direct aerobic metabolism of contaminants are not available, it may be difficult to show that this type of attenuation process is occurring.

It may also be uncertain whether co-metabolic reactions are occurring in the plume segment. To resolve this uncertainty, it may be necessary to perform a detailed analysis of contaminant loss down the centerline of the plume: i) to determine if the observed reduction in concentrations is due to dispersion only or due to a combination of dispersion and co-metabolic reactions; and ii) to determine if a primary substrate (e.g., phenol, methane, propane, etc.) is present in the plume segment. 


\begin{tabular}{|c|c|c|c|c|}
\hline $\begin{array}{c}\text { Simple } \\
\text { Fast }\end{array}$ & $\begin{array}{c}\text { Simple } \\
\text { Slow }\end{array}$ & $\begin{array}{c}\text { Heterogeneous } \\
\text { Fast }\end{array}$ & $\begin{array}{c}\text { Heterogeneous } \\
\text { Slow }\end{array}$ & $\begin{array}{c}\text { Fractured/ } \\
\text { Porous Rock }\end{array}$ \\
\hline
\end{tabular}

\begin{tabular}{|l|l|l|}
\hline Aerobic & Anoxic & Anaerobic \\
\hline
\end{tabular}

\section{HOW DO I ANALYZE DATA?}

In a slow-flowing aquifer, a plume is more likely to be shorter and will become stable (if it is going to) in a longer period of time than in faster-flow aquifers. Thus, plume maps, concentration vs. time at each well, and concentration vs. distance plots may be difficult to interpret to determine if the plume is expanding, stable, or shrinking. Longer and consistent temporal records of concentration at key wells (particularly at the leading edge of the plume) are important to evaluate MNA under the heterogeneous-slow flowing scenario. However, care must be taken to ensure that the monitoring network is sufficient for the heterogeneous conditions at the site. Because daughter products are not readily measured for aerobic reactions, additional information to confirm attenuation processes may be needed. Especially if the plume edge is close to receptors, it may be necessary to provide additional data to verify aerobic degradation processes are occurring and to assess the sustainability of these processes. Molecular probe data to verify the presence of the appropriate microorganisms and laboratory microcosm tests may provide this type of information. A more detailed geochemical analysis may also be warranted to assess sustainability.

Transport models can be very helpful in analyzing and visualizing the data and expected plume conditions for comparison to field data. Transport models may be needed to predict the future state of the plume so that decisions can be made in a timely fashion and then confirmed through the long-term monitoring portion of MNA implementation. A simple transport model such as BIOCHLOR can be helpful as a screening tool in analyzing and visualizing the data and expected plume conditions for comparison to field data. However, BIOCHLOR may not be sufficient to describe the flow conditions in a heterogeneous aquifer. As such, more complex numerical modeling is more likely to be needed as part of MNA evaluation for Scenario 12 sites. To support this more complex analysis, more detailed field measurements may be required. The chart below summarizes an approach for analyzing data at sites depending on whether the concentration data indicates that the plume is decreasing, stable, or increasing and the source type. As noted in the table, as the source gets stronger and the plume is less likely to be decreasing in extent, more information is needed to support selection of an MNA remedy.

\begin{tabular}{|c|c|c|c|}
\hline & \multicolumn{3}{|c|}{ PLUME STATUS } \\
\hline $\begin{array}{l}\text { CONTAMINANT } \\
\text { CONCENTRATIONS/GEOCHEMICAL } \\
\text { STATUS }\end{array}$ & $\begin{array}{l}\text { DECREASING OR } \\
\text { PROBABLY } \\
\text { DECREASING }\end{array}$ & STABLE & $\begin{array}{l}\text { INCREASING, } \\
\text { PROBABLY } \\
\text { INCREASING, OR } \\
\text { PERTURBED }^{1}\end{array}$ \\
\hline Weak Source & - Mass loss & $\begin{array}{ll}\text { - } & \text { Mass loss } \\
\text { - } & \text { Geochemical footprints } \\
\text { - } & \text { Simple model }\end{array}$ & $\begin{array}{l}\text { - } \text { Mass loss } \\
\text { - } \quad \text { Coochemical footprints } \\
\text { Special Studies }\end{array}$ \\
\hline Strong Source & $\begin{array}{l}\text { - Mass loss } \\
\text { - Geochemical footprints }\end{array}$ & $\begin{array}{ll}\text { - } & \text { Mass loss } \\
\text { - } & \text { Coochemical footprints } \\
& \text { Special Studies }\end{array}$ & $\begin{array}{l}\text { - } \text { Mass loss } \\
\text { - } \text { Geochemical footprints } \\
\text { - } \quad \text { Comprehensive Model/ } \\
\text { Special Studies }\end{array}$ \\
\hline
\end{tabular}

${ }^{1}$ For instance if the plume has been impacted by a previous remedy such as P\&T.

To demonstrate mass loss construct these graphics:

- Concentration vs. time plots at individual wells;

- Concentration vs. distance plots, with multiple lines for different sampling events through time;

- Plume maps showing plume extent at different times (i.e., either panel maps, or one map with several plume boundaries for different times).

To show geochemical footprints make tables or figures that show:

- Daughter product production from abiotic reactions;

- Presence of primary substrate for co-metabolic reactions;

- Chloride product (this may not work for many sites, however, due to background chloride);

- Moderate to high dissolved oxygen concentrations (shows geochemical conditions area OK);

- No or limited methane production (shows geochemical conditions area OK).

To perform modeling, typical tools include the following:

- Simple transport model (analytical model, e.g., BIOCHLOR, BIOBALANCE ${ }^{1}$ );

- Comprehensive transport model (numerical model, e.g., RT3D). 


\begin{tabular}{|c|c|c|c|c|c|}
\hline $\begin{array}{c}\text { Simple } \\
\text { Fast }\end{array}$ & $\begin{array}{c}\text { Simple } \\
\text { Slow }\end{array}$ & $\begin{array}{c}\text { Heterogeneous } \\
\text { Fast }\end{array}$ & $\begin{array}{c}\text { Heterogeneous } \\
\text { Slow }\end{array}$ & $\begin{array}{c}\text { Fractured/ } \\
\text { Porous Rock }\end{array}$ & $\begin{array}{c}\text { Aerobic } \\
\text { Anoerobic }\end{array}$ \\
\hline
\end{tabular}

If a special study is needed, some of the following may be applicable:

- Carbon/chlorine isotope analysis (indicator of degradation processes)

- Molecular probes (indicators of microbial activity)

- Microcosm tests (determine the reaction processes occurring at the site).

${ }^{1}$ Developed by Groundwater Services (www.gsi-net.com), with the support of the Savannah River National Laboratory and DOE, to evaluate monitored natural attenuation at chlorinated solvent sites.

\section{WHAT ABOUT COSTS AND ENHANCEMENTS?}

Costs for evaluating and implementing MNA for this scenario are primarily dependent on the following items.

- $\quad$ Source strength - Stronger and longer lasting sources will be more costly.

- $\quad$ Depth to the plume and size of the plume - Deeper, larger plumes require more and costlier monitoring wells.

- $\quad$ Travel time to the receptor - Plumes closer to receptors will require more frequent monitoring and over a long period of time with the slow groundwater flow rate.

- $\quad$ Plume stability - Less stable plumes require more rigorous evaluation and monitoring to select and implement MNA. With the slow groundwater flow rate, more rigorous evaluation or a longer period of monitoring may cause higher costs than for sites with a high groundwater flow rate.

- $\quad$ Nature of Heterogeneities - More heterogeneous aquifers may require a larger number of monitoring locations and more detailed analysis of flow and transport as part of evaluating MNA.

MNA may be a viable single remedy for the site. If it is determined that MNA may not meet remediation goals, a good first option is to evaluate the potential use of sustainable enhancements (enhanced attenuation). The objective being to adjust the attenuation conditions sufficiently such that the plume is controlled. If the enhancements are insufficient to control the plume, source control treatment may be required. In general, enhanced attenuation is less likely a viable option for a fast flow regime with a strong source, unless source treatment is undertaken initially. The sections below discuss options for enhanced attenuation and source control related to this scenario.

\section{Key Enhanced Attenuation Concepts}

Enhanced attenuation must be carefully designed in a heterogeneous flow system. However, targeted application of enhancements may be highly effective due to the slow groundwater flow conditions. Enhancements are presented organized by the different zones in which they may be applied: source zone (reduction of contaminant mass flux to plume); plume (enhanced attenuation processes); or discharge zone (enhanced attenuation processes). Within the source zone, enhancements can be applied as a hydraulic manipulation or as a passive source reduction (active source control is discussed in the next section). Within the plume and discharge zone, either biological (microbial or plant based) or abiotic (abiotic degradation, reactive barriers, sorption) attenuation processes can be enhanced. A description of potential enhanced attenuation approaches, and their applicability to Scenario 12 sites, is shown below. More detailed information about each technology listed below is available in Early et al., (2005). 


\begin{tabular}{|c|c|c|c|c|}
\hline $\begin{array}{c}\text { Simple } \\
\text { Fast }\end{array}$ & $\begin{array}{c}\text { Simple } \\
\text { Slow }\end{array}$ & $\begin{array}{c}\text { Heterogeneous } \\
\text { Fast }\end{array}$ & $\begin{array}{c}\text { Heterogeneous } \\
\text { Slow }\end{array}$ & $\begin{array}{c}\text { Fractured/ } \\
\text { Porous Rock }\end{array}$ \\
\hline
\end{tabular}

\begin{tabular}{|l|l|l|}
\hline Aerobic & Anoxic & Anaerobic \\
\hline
\end{tabular}

\section{Enhancement Summary}

\begin{tabular}{|c|c|c|}
\hline Enhancement & Description & Applicability to Scenario 12 Sites \\
\hline \multicolumn{3}{|c|}{ SOURCE ZONE ENHANCEMENTS } \\
\hline $\begin{array}{l}\text { Surface water or } \\
\text { groundwater } \\
\text { interception/diversion }\end{array}$ & $\begin{array}{l}\text { Use of interception } \\
\text { trenches or wells, } \\
\text { surface covers, or } \\
\text { phyto-covers (plants) } \\
\text { to reduce water flux } \\
\text { through source area }\end{array}$ & $\begin{array}{l}\text { May be difficult in heterogeneous conditions. Likely more } \\
\text { applicable using surface covers or phyto-covers if the } \\
\text { source is primarily within the vadose zone. }\end{array}$ \\
\hline Physical containment & $\begin{array}{l}\text { Use of grout walls and } \\
\text { other physical } \\
\text { containment }\end{array}$ & $\begin{array}{l}\text { Potentially applicable depending on the geometry of the } \\
\text { source zone. }\end{array}$ \\
\hline Passive extraction & $\begin{array}{l}\text { Use of passive soil } \\
\text { vapor extraction }\end{array}$ & Useful if the source is primarily within the vadose zone. \\
\hline $\begin{array}{l}\text { Enhanced } \\
\text { biodegradation }\end{array}$ & $\begin{array}{l}\text { Injection of long-term } \\
\text { dissolved oxygen } \\
\text { source }\end{array}$ & $\begin{array}{l}\text { Well suited for aerobic sites if oxygen concentrations are } \\
\text { marginal in some areas; enhances existing aerobic } \\
\text { biodegradation reactions. Complex hydrogeology can } \\
\text { make application difficult. Slow groundwater flow may } \\
\text { require relatively smaller amounts of oxygen source. }\end{array}$ \\
\hline $\begin{array}{l}\text { Enhanced } \\
\text { biodegradation }\end{array}$ & $\begin{array}{l}\text { Injection of electron } \\
\text { donor (e.g., HRC, } \\
\text { molasses, vegetable } \\
\text { oil) to enhance } \\
\text { microbial degradation } \\
\text { of the source. }\end{array}$ & $\begin{array}{l}\text { Typically more appropriate for anaerobic sites; stimulates } \\
\text { anaerobic contaminant biodegradation reactions. } \\
\text { Potentially useful at aerobic sites in source area to } \\
\text { convert contaminants such as PCE and TCE into } \\
\text { contaminants such as DCE and VC that are degradable } \\
\text { under aerobic conditions. Need careful control of process } \\
\text { to avoid depleting all of the oxygen for the plume and } \\
\text { eliminating the potential for aerobic reactions. Complex } \\
\text { hydrogeology can make application difficult. Slow } \\
\text { groundwater flow may require relatively smaller amounts } \\
\text { of electron donor. }\end{array}$ \\
\hline $\begin{array}{l}\text { Permeable reactive } \\
\text { barrier }\end{array}$ & $\begin{array}{l}\text { Use of zero valent } \\
\text { iron, reduced } \\
\text { sediment iron, } \\
\text { enhanced partitioning, } \\
\text { or biological barrier to } \\
\text { attenuate some of the } \\
\text { contaminant flux at } \\
\text { the downgradient } \\
\text { edge of the source } \\
\text { area. }\end{array}$ & $\begin{array}{l}\text { Barriers typically use anaerobic reactions. Influent of } \\
\text { dissolved oxygen is problematic for the barrier and the } \\
\text { aquifer down gradient of the barrier will be depleted in } \\
\text { oxygen. Thus, barriers that use anaerobic reactions are } \\
\text { not typically suitable for aerobic sites. May be more } \\
\text { difficult and expensive to construct due to heterogeneous } \\
\text { conditions. Slower groundwater flows potentially result in } \\
\text { less contaminant and competing electron acceptors } \\
\text { passing through the barrier, requiring less thickness to } \\
\text { achieve desired treatment levels. }\end{array}$ \\
\hline
\end{tabular}




\begin{tabular}{|c|c|c|c|c|}
\hline $\begin{array}{c}\text { Simple } \\
\text { Fast }\end{array}$ & $\begin{array}{c}\text { Simple } \\
\text { Slow }\end{array}$ & $\begin{array}{c}\text { Heterogeneous } \\
\text { Fast }\end{array}$ & $\begin{array}{c}\text { Heterogeneous } \\
\text { Slow }\end{array}$ & $\begin{array}{c}\text { Fractured/ } \\
\text { Porous Rock }\end{array}$ \\
\hline
\end{tabular}

\begin{tabular}{|l|l|l|}
\hline Aerobic & Anoxic & Anaerobic \\
\hline
\end{tabular}

\begin{tabular}{|c|c|c|}
\hline Enhancement & Description & Applicability to Scenario 12 Sites \\
\hline \multicolumn{3}{|c|}{ PLUME AND DISCHARGE ZONE ENHANCEMENTS } \\
\hline $\begin{array}{l}\text { Enhanced } \\
\text { biodegradation }\end{array}$ & $\begin{array}{l}\text { Injection of long-term } \\
\text { dissolved oxygen } \\
\text { source }\end{array}$ & $\begin{array}{l}\text { Well suited for aerobic sites if oxygen concentrations are } \\
\text { marginal in some areas; enhances existing aerobic } \\
\text { biodegradation reactions. Complex hydrogeology can } \\
\text { make application difficult. Slow groundwater flow may } \\
\text { require relatively smaller amounts of oxygen source. }\end{array}$ \\
\hline $\begin{array}{l}\text { Enhanced } \\
\text { biodegradation }\end{array}$ & $\begin{array}{l}\text { Injection of electron } \\
\text { donor (e.g., HRC, } \\
\text { molasses, vegetable } \\
\text { oil) to enhance } \\
\text { microbial degradation } \\
\text { of the source. }\end{array}$ & $\begin{array}{l}\text { Typically more appropriate for anaerobic sites; stimulates } \\
\text { anaerobic contaminant biodegradation reactions. } \\
\text { Potentially useful at aerobic sites in source area to } \\
\text { convert contaminants such as PCE and TCE into } \\
\text { contaminants such as DCE and VC that are degradable } \\
\text { under aerobic conditions. Need careful control of process } \\
\text { to avoid depleting all of the oxygen for the plume and } \\
\text { eliminating the potential for aerobic reactions. Complex } \\
\text { hydrogeology can make application difficult. Slow } \\
\text { groundwater flow may require relatively smaller amounts } \\
\text { of electron donor. }\end{array}$ \\
\hline $\begin{array}{l}\text { Permeable reactive } \\
\text { barrier }\end{array}$ & $\begin{array}{l}\text { Use of zero valent } \\
\text { iron, reduced } \\
\text { sediment iron, } \\
\text { enhanced partitioning, } \\
\text { or biological barrier to } \\
\text { attenuate some of the } \\
\text { contaminant flux } \\
\text { within the plume. }\end{array}$ & $\begin{array}{l}\text { Barriers typically use anaerobic reactions. Influent of } \\
\text { dissolved oxygen is problematic for the barrier and the } \\
\text { aquifer down gradient of the barrier will be depleted in } \\
\text { oxygen. Thus, barriers that use anaerobic reactions are } \\
\text { not typically suitable for aerobic sites. May be more } \\
\text { difficult and expensive to construct due to heterogeneous } \\
\text { conditions. Slower groundwater flows potentially result in } \\
\text { less contaminant and competing electron acceptors } \\
\text { passing through the barrier, requiring less thickness to } \\
\text { achieve desired treatment levels. }\end{array}$ \\
\hline Phytoextraction & $\begin{array}{l}\text { Use of plants to } \\
\text { extract contaminants } \\
\text { from near surface } \\
\text { groundwater }\end{array}$ & $\begin{array}{l}\text { Heterogeneous aquifer conditions may make application } \\
\text { difficult. }\end{array}$ \\
\hline $\begin{array}{l}\text { Plant-based hydraulic } \\
\text { control (plume } \\
\text { enhancement only) }\end{array}$ & $\begin{array}{l}\text { Use of plants to } \\
\text { control hydraulic } \\
\text { gradient and slow } \\
\text { groundwater }\end{array}$ & $\begin{array}{l}\text { Heterogeneous aquifer conditions may make application } \\
\text { difficult. }\end{array}$ \\
\hline
\end{tabular}




\begin{tabular}{|c|c|c|c|c|}
\hline $\begin{array}{c}\text { Simple } \\
\text { Fast }\end{array}$ & $\begin{array}{c}\text { Simple } \\
\text { Slow }\end{array}$ & $\begin{array}{c}\text { Heterogeneous } \\
\text { Fast }\end{array}$ & $\begin{array}{c}\text { Heterogeneous } \\
\text { Slow }\end{array}$ & $\begin{array}{c}\text { Fractured/ } \\
\text { Porous Rock }\end{array}$ \\
\hline
\end{tabular}

\begin{tabular}{|l|c|c|}
\hline \multicolumn{3}{|c}{ GEOCHEMICAL SETTING } \\
\hline Aerobic & Anoxic & Anaerobic \\
\hline
\end{tabular}

\section{Key Source Control Concepts}

A description of potential source control measures, and their applicability to Scenario 12 sites, is shown below. Note that source control measures are unlikely to achieve complete restoration at a site, and some source material is always left behind after treatment (U.S. EPA, 2003; McGuire et al., 2006).

\begin{tabular}{|c|c|c|c|}
\hline Example Technology & $\begin{array}{l}\text { Performance } \\
\left(25^{\text {th }}-75^{\text {th }} \text { Percentile }\right. \\
\% \text { reduction in } \\
\text { parent compound })^{1}\end{array}$ & $\begin{array}{l}\frac{\text { Unit Cost }}{25^{\text {th }}-75^{\text {th }}} \\
\text { Percentile } \\
\text { (\$/yrd })^{2}\end{array}$ & Applicability to Scenario 12 Sites \\
\hline In-situ biodegradation & $73-99$ & $27-152$ & $\begin{array}{l}\text { Potentially well suited for aerobic sites through } \\
\text { addition of co-substrate for aerobic degradation or } \\
\text { potentially through use of anaerobic reactions } \\
\text { depending on how this action impacts the } \\
\text { downgradient geochemical conditions. Complex } \\
\text { hydrogeology can make application difficult. Slow } \\
\text { groundwater flow may require relatively smaller } \\
\text { amounts of electron donor. Least expensive } \\
\text { treatment option. }\end{array}$ \\
\hline Thermal treatment & $68-99.9$ & $48-129$ & $\begin{array}{l}\text { Does not appear to disrupt MNA after treatment. } \\
\text { Heterogeneous aquifer conditions may make } \\
\text { application difficult. }\end{array}$ \\
\hline Chemical oxidation & $70-97$ & $47-194$ & $\begin{array}{l}\text { Removes more total CVOCs than enhanced } \\
\text { biodegradation but shows more rebound }{ }^{1} \text {. } \\
\text { Heterogeneous aquifer conditions may make } \\
\text { application difficult. May be suitable for aerobic } \\
\text { sites. }\end{array}$ \\
\hline Surfactant/cosolvents & $92-98$ & $118-1322$ & $\begin{array}{l}\text { High treatment efficiency but much higher cost. } \\
\text { Costs reflect some expensive pilot-scale projects. } \\
\text { Heterogeneous aquifer conditions may make } \\
\text { application difficult. Some surfactants/cosolvents } \\
\text { can serve as electron donors for subsequent } \\
\text { anaerobic biodegradation reactions. }\end{array}$ \\
\hline Air sparging & - & - & $\begin{array}{l}\text { May be a viable alternative depending on the site } \\
\text { geology (e.g., contamination in an unconfined } \\
\text { aquifer). Heterogeneous aquifer conditions may } \\
\text { make application difficult. }\end{array}$ \\
\hline $\begin{array}{l}\text { Pump and Treat source } \\
\text { containment }\end{array}$ & NA & & $\begin{array}{l}\text { This approach does not reduce mass significantly } \\
\text { compared to the rate of mass loss without P\&T } \\
\text { and may need to be operated for a long time. }\end{array}$ \\
\hline
\end{tabular}


HYDROGEOLOGIC SETTING

\begin{tabular}{|c|c|c|c|c|}
\hline $\begin{array}{c}\text { Simple } \\
\text { Fast }\end{array}$ & $\begin{array}{c}\text { Simple } \\
\text { Slow }\end{array}$ & $\begin{array}{c}\text { Heterogeneous } \\
\text { Fast }\end{array}$ & $\begin{array}{c}\text { Heterogeneous } \\
\text { Slow }\end{array}$ & $\begin{array}{c}\text { Fractured/ } \\
\text { Porous Rock }\end{array}$ \\
\hline
\end{tabular}

GEOCHEMICAL SETTING

\section{SCENARIO NUMBER 13}

\section{Flow in Fractured or Porous Rock}

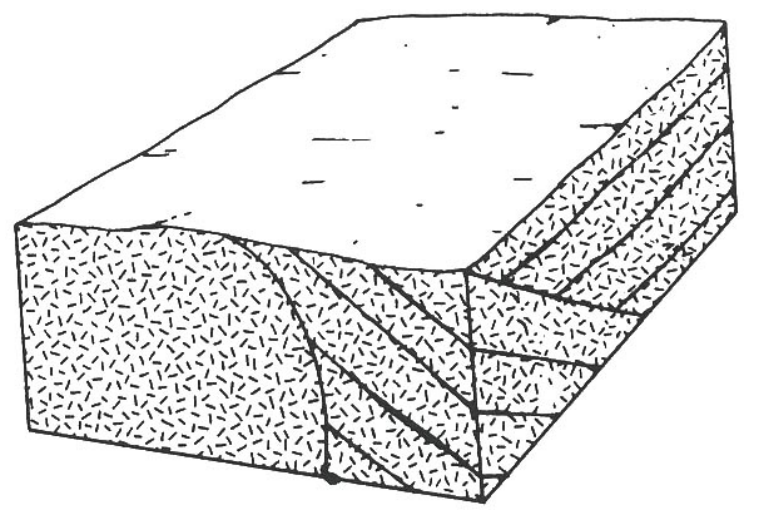




\begin{tabular}{|c|c|c|c|c|}
\hline $\begin{array}{c}\text { Simple } \\
\text { Fast }\end{array}$ & $\begin{array}{c}\text { Simple } \\
\text { Slow }\end{array}$ & $\begin{array}{c}\text { Heterogeneous } \\
\text { Fast }\end{array}$ & $\begin{array}{c}\text { Heterogeneous } \\
\text { Slow }\end{array}$ & $\begin{array}{c}\text { Fractured/ } \\
\text { Porous Rock }\end{array}$ \\
\hline
\end{tabular}

\section{AII}

\section{SCENARIO 13 DESCRIPTION: FLOW IN FRACTURED OR POROUS ROCK}

The hydrogeologic setting below defines the basic scenario type. The geochemical condition is also important, but is a secondary factor in determining the approach for MNA. This scenario has the following characteristics:

\section{Hydrogeologic Setting}

"Flow in Fractured or Porous Rock" Hydrogeology:

- Flow patterns dominated by fracture characteristics

- Groundwater velocity and corresponding contaminant velocity can be high even for low volumetric flow rates.

- $\quad$ Nature of the rock matrix and secondary porosity are important in understanding the impact of sorption and diffusion on contaminant transport.

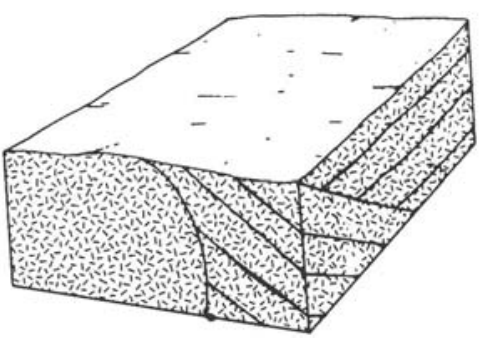

Block Diagram of Bedrock Uplands Aquifer from DRASTIC System

\section{Geochemical Setting}

Geochemistry:

- Geochemistry is important, but secondary to hydrogeologic setting in determining approach for MNA evaluation.

- Once the hydrogeologic nature of the site is defined, the geochemistry-related MNA considerations from other scenarios can be used to continue the MNA evaluation approach 


\begin{tabular}{|c|c|c|c|c|}
\hline $\begin{array}{c}\text { Simple } \\
\text { Fast }\end{array}$ & $\begin{array}{c}\text { Simple } \\
\text { Slow }\end{array}$ & $\begin{array}{c}\text { Heterogeneous } \\
\text { Fast }\end{array}$ & $\begin{array}{c}\text { Heterogeneous } \\
\text { Slow }\end{array}$ & $\begin{array}{c}\text { Fractured/ } \\
\text { Porous Rock }\end{array}$ \\
\hline
\end{tabular}

\section{EFFECT OF MODIFYING FACTORS}

\begin{tabular}{|c|c|}
\hline MODIFYING FACTOR & KEY POINTS \\
\hline Source $S$ & $\begin{array}{l}\text { IF YOU HAVE A STRONG SOURCE: } \\
\text { - Plumes (both parent and daughter compounds) may be longer } \\
\text { - Source zones may persist for longer periods of time } \\
\text { IF YOU HAVE A MODERATE SOURCE: } \\
\text { - Intermediate condition between Strong and Weak Source } \\
\text { IF YOU HAVE A WEAK SOURCE: } \\
\text { - Plumes may be shorter although fracture heterogeneity may be a more } \\
\text { - } \quad \text { Sominant factor for plume length } \\
\text { - } \quad \text { significantly impacted by the nature of secondary porosity } \\
\text { MNA alone or MNA with EA more likely to be sufficient }\end{array}$ \\
\hline Source & $\begin{array}{l}\text { IF YOU HAVE MOSTLY A VADOSE ZONE SOURCE: } \\
\text { - Source may appear small due to dilution but can be large } \\
\text { - Plumes will be thinner and closer to water table } \\
\text { IF YOU HAVE A SUBMERGED SOURCE: } \\
\text { - Complex hydrogeology means matrix diffusion may be important } \\
\text { - Source mass flux can decrease relatively rapidly as DNAPL fingers } \\
\text { - dissolve } \\
\text { IF YOU HAVE A MIXED SOURCE: } \\
\text { - May have combination of the factors above }\end{array}$ \\
\hline Travel Time to Re & $\begin{array}{l}\text { CLOSE RECEPTORS (<2 YEARS TRAVEL TIME) } \\
\text { More intensive monitoring system likely to be needed due to potential } \\
\text { - } \\
\text { Merious consequences in event of failure of MNA/EA. } \\
\text { MODERATE RECEPTORS ( }>2 \text { but < } 5 \text { YEARS TRAVEL TIME) } \\
\text { - Represents middle-ground case } \\
\text { DISTANT RECEPTORS (>5 YEARS TRAVEL TIME) } \\
\text { - Less intensive monitoring system likely to be needed due to lower } \\
\text { potential for serious consequences in event of failure of MNA/EA. } \\
\text { MNA alone or MNA with EA more likely to be sufficient } \\
\text { *Travel time is an important factor, but may be significantly impacted by the nature } \\
\text { of fracturing, and therefore a more sophisticated analysis may be necessary }\end{array}$ \\
\hline Plume Stability & $\begin{array}{l}\text { EXPANDING OR PERTURBED }{ }^{1} \text { PLUME } \\
\text { - More intensive monitoring system likely to be needed } \\
\text { STABLE PLUME } \\
\text { SHRINKING PLUME } \\
\text { - Lepresents middle-ground case } \\
\text { - MNA alone likely to be sufficient } \\
{ }^{1} \text { For instance if the plume has been impacted by a previous remedy such as P\&T. }\end{array}$ \\
\hline
\end{tabular}




\begin{tabular}{|c|c|c|c|c|}
\hline $\begin{array}{c}\text { Simple } \\
\text { Fast }\end{array}$ & $\begin{array}{c}\text { Simple } \\
\text { Slow }\end{array}$ & $\begin{array}{c}\text { Heterogeneous } \\
\text { Fast }\end{array}$ & $\begin{array}{c}\text { Heterogeneous } \\
\text { Slow }\end{array}$ & $\begin{array}{c}\text { Fractured/ } \\
\text { Porous Rock }\end{array}$ \\
\hline
\end{tabular}

\section{WILL MNA WORK?}

\section{Potential for MNA Processes to Control Plume}

The nature of the flow system has a significant impact on the ability of MNA to control a plume in a fractured or porous rock setting. First, the linear velocity of the groundwater, and therefore, contaminants may be fast depending on the nature of the fractures or porosity. In this case, attenuation rates would also need to be high stabilize the plume. Second, attenuation in terms of sorption and dispersion/diffusion in a fractured or porous rock setting is highly dependent on the nature of secondary fractures or porosity in the rock matrix. The secondary fractures or porosity act as "dead end" porosity because they do not create a connected flow path for contaminants. In some rock matrices, secondary fractures/porosity may provide a high capacity for contaminant movement into the matrix when contaminant concentration in the primary fractures/porosity is high. The effect of this movement will attenuate the plume similar to how sorption attenuates a plume in unconsolidated porous media. When contaminant concentrations in the primary fractures/porosity decreases, the secondary fractures/porosity will cause long term tailing of a plume due to slow diffusion out of the rock matrix. Potentially, the tailing of the plume may be of concern if the matrix diffusion maintains concentrations in the primary fractures/porosity higher than the remediation action level. Based on these phenomena for fractured or porous rock settings, the most important feature controlling whether MNA will be a suitable remedy is the nature of the fractures/porosity and corresponding rock matrix.

\section{Key Sustainability Concept}

Sustainability of MNA will be primarily related to the geochemical setting and reactive degradation/transformation of the contaminants (see Scenarios 7, 8, and 9 for discussions related to geochemical factors). However, sustainability may need to also consider the potential for significant initial attenuation of a plume while matrix diffusion is removing contaminant from the primary porosity that leads to long-term tailing of a plume. The tailing of the plume must be considered in terms of whether the concentrations will be held at a level above the remediation goal. 


\begin{tabular}{|c|c|c|c|c|}
\hline $\begin{array}{c}\text { Simple } \\
\text { Fast }\end{array}$ & $\begin{array}{c}\text { Simple } \\
\text { Slow }\end{array}$ & $\begin{array}{c}\text { Heterogeneous } \\
\text { Fast }\end{array}$ & $\begin{array}{c}\text { Heterogeneous } \\
\text { Slow }\end{array}$ & $\begin{array}{c}\text { Fractured/ } \\
\text { Porous Rock }\end{array}$ \\
\hline
\end{tabular}$\quad$\begin{tabular}{|c|c|c|}
\hline All \\
\hline
\end{tabular}

\section{HOW DO I CHARACTERIZE THIS TYPE OF SITE?}

\section{Actions Needed to Determine MNA Viability}

The following are key considerations for evaluating the viability of MNA at a Flow in Fractured or Porous Rock type site:

- Assess the nature of the flow system including primary and secondary fractures/porosity and the nature of the rock matrix. In many cases, it may be possible to conceptually model the fracture/porosity system using a continuum approach such that descriptions of flow and attenuation are similar to those used for unconsolidated porous media. With this type of conceptual model, contaminant fate and transport can be evaluated using a dual domain model or an alternatively with a transfer function model. In other cases, the nature of the fractures/porosity are not suitable for this type of conceptual model and it may not be possible to evaluate MNA with the traditional tools because the flow system cannot be adequately defined. If the site cannot be well represented or predicted in a reliable manner with any modeling approach, the requirements for monitoring and field documentation are increased.

- determine the relative horizontal and vertical plume movement and whether there are major fractures/porosity where the plume movement is significantly greater than in other parts of the aquifer;

- both parent compound and daughter compounds need to be delineated (the extent depends on the geochemical conditions);

- determine if plume(s) are expanding/perturbed, stable, or shrinking;

- determine the location of any receptors (if present) and determine the travel time from the edge of the plume to these receptors;

- a mass-balance type evaluation of the source zone may be possible, but care should be used in using this analysis depending on how well the flow system is understood.

\section{Key Monitoring Concepts}

A more extensive monitoring system will likely be required to delineate the plume, because wells information may only be representative of conditions in a small portion of the aquifer for a fractured or porous rock system.

\section{Key Uncertainty Concepts}

A key uncertainty for this scenario is associated with knowing that the flow system is adequately understood and that the plume is sufficiently delineated. In a fractured or porous rock flow system, appropriate selection of monitoring locations is more difficult both horizontally and vertically within the plume. Because all of the MNA evaluation methods rely on a suitable conceptual model and data to describe the plume and subsurface properties, more data is typically required in a fractured or porous rock flow system to complete the evaluation at a level of detail acceptable to decision makers. There may be other uncertainties depending on the geochemical setting and source characteristics (see Scenarios 7, 8, and 9 for discussions related to geochemical factors). 


\begin{tabular}{|c|c|c|c|c|}
\hline $\begin{array}{c}\text { Simple } \\
\text { Fast }\end{array}$ & $\begin{array}{c}\text { Simple } \\
\text { Slow }\end{array}$ & $\begin{array}{c}\text { Heterogeneous } \\
\text { Fast }\end{array}$ & $\begin{array}{c}\text { Heterogeneous } \\
\text { Slow }\end{array}$ & $\begin{array}{c}\text { Fractured/ } \\
\text { Porous Rock }\end{array}$ \\
\hline
\end{tabular}

\section{HOW DO I ANALYZE DATA?}

In a fractured or porous rock flow system, data analysis technique is strongly dependent on the nature of the flow system and the ability of well information to adequately reflect the plume conditions. As such, general approaches for data analysis are not discussed in this scenario. Instead, it is suggested that technical expertise specific to these types of flow systems and corresponding natural attenuation processes is necessary to conduct the data analysis. Potentially, transport models can be very helpful in analyzing and visualizing the data and expected plume conditions for comparison to field data. Transport models may be needed to predict the future state of the plume so that decisions can be made in a timely fashion and then confirmed through the long-term monitoring portion of MNA implementation. A simple transport model such as BIOCHLOR will likely not be sufficient to describe the flow conditions. More complex numerical modeling is more appropriate. The data analysis will also need to consider the geochemical system as described for the other scenarios (see scenarios 7, 8, and9). In particular, the data analysis will also need to include an appropriate assessment of contaminant behavior in the secondary fractures/porosity and associated contaminant sorption/desorption processes.

\section{WHAT ABOUT COSTS AND ENHANCEMENTS?}

Costs for evaluating and implementing MNA for this scenario are primarily dependent on the following items.

- Nature of fractured or porous rock flow system - The complexity of the flow system will likely be the predominant factor in determining the costs for evaluating and implementing MNA.

- Source strength - Stronger and longer lasting sources will be more costly especially due to the high groundwater flow rate.

- Depth to the plume and size of the plume - Deeper, larger plumes require more and costlier monitoring wells.

- Travel time to the receptor - Plumes closer to receptors will require more frequent monitoring especially with the high groundwater flow rate.

- Plume stability - Less stable plumes require more rigorous evaluation and monitoring to select and implement MNA especially with the high groundwater flow rate.

- Extent of variability in geochemical conditions - More variability will likely require more characterization and monitoring to assess attenuation conditions within each different geochemical zone.

While MNA may be difficult to evaluate as a viable single remedy for the site, it may also be difficult to design and implement due to the challenges of working in a fractured or porous rock system. If it is determined that MNA may not meet remediation goals, a good first option is to evaluate the potential use of sustainable enhancements (enhanced attenuation). The objective being to adjust the attenuation conditions sufficiently such that the plume is controlled. If the enhancements are insufficient to control the plume, source control treatment may be required. In general, enhanced attenuation is less likely a viable option for a fast flow regime with a strong source, unless source treatment is undertaken initially. The type of enhanced attenuation of source control approaches that are appropriate are dependent on the geochemical conditions in addition to the complexity of the flow system. Potentially applicable approaches for a fast-flow heterogeneous system are described in scenarios 7, 8, and 9. This information can be consulted as a starting point for evaluating enhancements or source control. However, more so than for other scenarios, a site specific assessment of remediation approaches is warranted. 